\title{
Pareamento de Elétrons por Intermédio de um Bipolaron em Supercondutores com Alta Temperatura de Transição
}

\author{
Alan Ramer dos Santos \\ DissertaÇÃo APRESENTADA \\ $\mathrm{AO}$ \\ Instituto DE Matemática e Estatística \\ DA \\ Universidade DE SÃo PAUlo \\ PARA \\ OBTENÇÃO DO TÍTULO \\ $\mathrm{DE}$ \\ Mestre em CiênCIAS
}

Programa: Matemática Aplicada

Orientador: Prof. Dr. Walter Alberto de Siqueira Pedra

Durante o desenvolvimento deste trabalho o autor recebeu auxílio financeiro do CNPq, vigente no processo: $131702 / 2018-1$

São Paulo, março de 2020 


\section{Pareamento de Elétrons por Intermédio de um Bipolaron em Supercondutores com Alta Temperatura de Transição}

Esta versão da dissertação contém as correções e alterações sugeridas pela Comissão Julgadora durante a defesa da versão original do trabalho, realizada em 29/07/2020. Uma cópia da versão original está disponível no

Instituto de Matemática e Estatística da Universidade de São Paulo.

Comissão Julgadora:

- Prof. Dr. Walter Alberto de Siqueira Pedra (orientador) - IF-USP

- Prof. Dr. Severino Toscano do Rêgo Melo - IME-USP

- Prof. Dr. César Rogério de Oliveira - UFSCar 


\section{Resumo}

SANTOS, A. R. Pareamento de Elétrons por Intermédio de um Bipolaron em Supercondutores com Alta Temperatura de Transição. 2020. 126 f. Dissertação (Mestrado) - Instituto de Matemática e Estatística, Universidade de São Paulo, São Paulo, 2020.

Com base em uma classe de modelos microscópicos para supercondutores com alta temperatura de transição proposto nos artigos [BdPdSP15] e [BdSPDdP19], analisamos rigorosamente um problema específico de três corpos relativo à dois férmions (elétrons) e um bóson (bipolaron) na rede cristalina de um determinado material.

Estudaram-se as propriedades espectrais de um Hamiltoniano do tipo Hubbard que leva em conta interações bipolaron-elétron, buscando num primeiro momento critérios que conduzam a formação de pares ligado (típicamente $d$-wave) na parte baixa de seu espectro.

Posteriormente, explora-se de forma analítica o comportamento efetivo da repulsão eletrônica no regime de hard-core, e com isso formalizamos um modelo efetivo (de baixa energia) com o fim de possibilitar uma abordagem mais simples ao efeito coletivo de supercondução.

Encerramos o trabalho investigando o aparecimento de alguns canais de espalhamento.

Palavras-chave: Supercondutores Não-Convencionais, Pareamento $d$-Wave, Análise Espectral, Teoria de Espalhamento. 



\section{Abstract}

SANTOS, A. R. Electrons Pairing Intermediated by a Bipolaron in High Transition Temperature Superconductors. 2020. 126 f. Dissertação (Mestrado) - Instituto de Matemática e Estatística, Universidade de São Paulo, São Paulo, 2020.

Based on a class of microscopic models for high-temperature superconductors proposed in the articles [BdPdSP15] and [BdSPDdP19], we rigorously analyze a specific three-body problem related to two fermions (electrons) and one boson (bipolaron) in the crystal lattice of a given material.

We studied the spectral properties of a Hubbard-like Hamiltonian which takes into account bipolaronelectron interactions, initially looking for criteria that leads to the formation of bound pairs (typically $d$-wave) at the bottom of its spectrum.

Subsequently, we derived by analytic methods the effective behavior of the electronic repulsion at the hard-core regime, and with that we formalized a low energy effective model in order to simplify the study of the collective effect of superconduction.

We conclude this work by investigating the appearance of some scattering channels.

Keywords: Non-Conventional Superconductors, $d$-Wave Pairing, Spectral Analysis, Scattering Theory. 



\section{Conteúdo}

I Fundamentos Matemáticos $\quad 21$

1 Tópicos em Análise Espectral 23

1.1 As Medidas Espectrais . . . . . . . . . . . . . . . . . . . . . . . . . . . . . . . . . . . 23

1.2 Critério de Weyl . . . . . . . . . . . . . . . . . . . . . . . . . . . . 36

1.3 Princípio de Birman-Schwinger . . . . . . . . . . . . . . . . . . . . . . . . . . . . . . 43

2 Integral Direta de Von Neumann $\quad 45$

2.1 Espaços $L^{p}$ Generalizados . . . . . . . . . . . . . . . . . . . . . . . . . . . . . . . . . . . . . . . . . . . . . .

2.2 Integral Direta de um Espaço de Hilbert . . . . . . . . . . . . . . . . . . . . . . . 51

2.3 Operadores Decomponíveis em Fibras . . . . . . . . . . . . . . . . . . . . . . . . 53

3 Mecânica Quântica $\quad 61$

3.1 Segunda Quantização . . . . . . . . . . . . . . . . . . . . . . . . 61

3.2 Operadores de Onda de Kato . . . . . . . . . . . . . . . . . . . . . . 70

II Estudo Microscópico de Supercondutores com Alta Temperatura de Transição

4 Um Modelo de Pareamento em Supercondutores Não-Convencionais $\quad 79$

4.1 Hamiltoniano do Tipo Hubbard com Termo de Interação Bipolaron-Elétron . . . . . . . . . 80

4.2 Decomposição em Fibras do Setor 2-Férmion-1-Bóson com Spin Total Zero . . . . . . . . 84

4.3 Espectro das Fibras de $\hat{H}$. . . . . . . . . . . . . . . . . . . . . . . . . . . . 92

4.4 Relação de Dispersão de Pares Ligado com Baixa Energia . . . . . . . . . . . . . . . . . . . 99

5 Comportamento Efetivo no Limite de Hard-Core da Repulsão Eletrônica 103

5.1 Representando $\mathfrak{T}$ em Termos de Integrais Explícitas . . . . . . . . . . . . . . . . . . . 103

5.2 Modelo Efetivo de Baixa Energia para o Limite de Hard-Core . . . . . . . . . . . . . . . 106

6 Canais de Espalhamento 111

6.1 Canal de Pares Eletrônicos Não-Ligado . . . . . . . . . . . . . . . . . . . . . . . . . . . 111

6.2 Canal de Pareamento $d$-Wave . . . . . . . . . . . . . . . . . . . . . . . . . . . . . . 113

$\begin{array}{ll}\text { A Resultados Suplementares } & 119\end{array}$ 


\section{Dicionário de Convenções e Notação}

No cálculo de predicados, se $P$ e $Q$ denotam duas proposições quaisquer, convencionaremos que

$$
P \doteq Q
$$

significa "P é por definição Q". Também, e com menor frequência, revela-se útil denotar

$$
P \equiv Q
$$

significando que os símbolos $P$ e $Q$ designam o mesmo objeto matemático. As figuras à seguir indica:

$$
\begin{aligned}
& \square=\text { fim de uma demonstração } \\
& \diamond=\text { fim de um exemplo } \\
& \bowtie=\text { fim de uma nota histórica }
\end{aligned}
$$

Os termos família e conjunto serão tidos como sinônimos, usados ambos para designar coleções de objetos. Se $S$ é um conjunto qualquer, subconjunto de um conjunto universal $Z$ fixo, se denotará por:

$$
\begin{aligned}
& |S|=\text { cardinal de } S \\
& \mathbb{P}(S)=\text { conjunto potência (ou partes) de } S \\
& \varnothing=\text { conjunto vazio } \\
& \text { id }=\text { função identidade } \\
& \chi_{S}=\text { função característica (ou indicadora) de } S
\end{aligned}
$$

Uma sequência finita de números inteiros não-negativos $I=\left(i_{1}, \ldots, i_{r}\right)$ será chamado de um multi-índice de ordem $r$, ou simplificadamente, uma $r$-lista. Seu comprimento será designado pelo símbolo $|I|=i_{1}+\cdots+i_{r}$, e diremos que ela é ordenada quando $i_{1}<\cdots<i_{r}$.

Denotaremos por $\mathbb{N}$ ao conjunto indutivo e bem-ordenado dos números naturais (sem o zero!). Estas duas propriedades serão usadas sob a forma de demonstrações por indução. Quando a inclusão do número 0 se mostrar necessária, escreveremos $\mathbb{N}_{0}$, diferentemente do que é sugerido no padrão ISO-31-11 (dedicado a sinais e símbolos matemáticos).

Também denotaremos com $\mathbb{Z}$ ao anel de integridade dos números inteiros, com $\mathbb{Q}$ ao corpo ordenado dos números racionais, com $\mathbb{R}$ ao corpo ordenado e completo dos números reais, com $\mathbb{C}$ ao corpo algebricamente fechado dos números complexos, em cujo caso $\operatorname{Re}(z)$ e $\operatorname{Im}(z)$ denotam (respectivamente) a parte real e imaginária do número complexo $z$, e por fim $\mathbb{K}$ para designar os dois ultimos. Se $K$ qualifica um corpo, $X$ um conjunto qualquer, $G$ um grupo e $B$ uma matriz quadrada com entradas em $K$, se denotará:

$$
\begin{aligned}
& \mathrm{M}_{n}(K)=\text { conjunto das matrizes } n \times n \text { com entradas em } K \\
& B^{t}=\text { matriz transposta de } B
\end{aligned}
$$




$$
\begin{aligned}
& K[t]=\text { conjunto dos polinômios em } K \\
& \operatorname{det}(B)=\text { determinante de } B \\
& \mathcal{S}(X)=\text { grupo simétrico de } X \\
& \mathcal{A}(X)=\text { grupo alternante de } X \\
& \operatorname{sgn}(\sigma)=\text { sinal de uma permutação } \sigma \in \mathcal{S}(X) \\
& \mathcal{S}_{n}=\mathcal{S}(\{1, \ldots, n\}) \\
& \mathcal{A}_{n}=\mathcal{A}(\{1, \ldots, n\})
\end{aligned}
$$

Se $X$ é um espaço topológico e $A \subseteq X$, escreveremos:

$$
\begin{aligned}
& \bar{A}=\text { fecho de } A \\
& \operatorname{int}(A)=\text { interior de } A \\
& \partial A=\text { fronteira topológica de } A \\
& \mathrm{C}(X)=\text { conjunto das funções contínuas de } X \text { em } \mathbb{C} \\
& \mathrm{C}_{c}(X)=\text { conjunto das funções contínuas de } X \text { em } \mathbb{C} \text { com suporte compacto } \\
& \mathfrak{B}_{X}=\sigma \text {-álgebra de Borel de } X \\
& \mathfrak{B}_{(X)}=\text { funções borelianas de } X \text { em } \mathbb{C} \\
& \mathfrak{B}_{\mathrm{c}}(X)=\text { funções borelianas de } X \text { em } \mathbb{C} \text { limitadas nos compactos de } X
\end{aligned}
$$

Se $(X, \mathcal{M}, \mu)$ e $(Y, \mathcal{N}, \nu)$ configuram dois espaços de medida, denota-se:

$$
\begin{aligned}
& \mathcal{M} \uparrow Z=\sigma \text {-álgebra induzida em } Z \subseteq X \text { por } \mathcal{M} \\
& \|\cdot\|_{p}=\text { norma usual em } \mathrm{L}^{p}, p \in[1, \infty] \\
& \mathfrak{m}=\text { medida de Lebesgue em } \mathbb{R} \\
& \mathfrak{c}=\text { medida de contagem } \\
& \mathcal{M} \otimes \mathcal{N}=\text {-álgebra produto de } \mathcal{M} \text { por } \mathcal{N} \\
& \mu \otimes \nu \\
& \Sigma(\mathcal{M})=\text { medida produto de } \mu \text { com } \nu \\
& \operatorname{im} \operatorname{ess}(f)=\text { imaģões } \mathcal{M} \text {-mensuráveis de } X \text { em } \mathbb{C} \\
& \mathscr{L}\left(\mathbb{R}^{d}\right)=\text { Lebessencial de } f \in \Sigma(\mathcal{M})
\end{aligned}
$$




$$
\mathscr{L}(G)=\quad \mathscr{L}\left(\mathbb{R}^{d}\right) \mid G, G \subseteq \mathbb{R}^{d}
$$

Sejam $X$ e $Y$ dois $K$-espaços vetoriais normados e $T$ uma transformação linear de $X$ em $Y$. Dizemos que $W$ é subespaço de $X$ escrevendo simplesmente $W \leq X$. De resto:

$$
\begin{aligned}
& \mathrm{L}(X, Y)=\text { espaço das transformações lineares de } X \text { em } Y \\
& \mathcal{B}(X, Y)=\text { espaço das transformações lineares e limitadas de } X \text { em } y \\
& \mathrm{~K}(X, Y)=\text { espaço dos operadores compactos de } X \text { em } Y \\
& \operatorname{Ker}(T)=\text { núcleo de } T \\
& \operatorname{Im}(T)=\text { imagem de } T \\
& X^{*}=\text { dual topológico de } X \\
& X^{* *}=\text { bidual topológico de } X \\
& 0 \\
& \langle U\rangle=\text { vetor nulo de } X \\
& \mathbb{S}^{1}(X)=\text { esfera unitária de } X \\
& \operatorname{dim}(X)=\text { dimensão algébrica de } X \\
& \mathrm{~d}(x, U)=\text { distância de } x \in X \text { ao conjunto } U, U \subseteq X \\
& \mathbf{B}_{\varepsilon}(x)=\text { bola aberta de raio } \varepsilon>0 \text { e centro em } x \in X \\
& {[T]_{\mathcal{B}, \mathcal{C}}=\text { matriz de } T \text { relativa as bases } \mathcal{B} \text { de } X \text { e } \mathcal{C} \text { de } Y .} \\
& \|\cdot\|_{X}=\text { norma de } X \\
& \|\cdot\|_{\text {op }}=\text { norma de operador } \\
& \|\cdot\|_{\infty}=\text { norma da convergência uniforme } \\
& \varkappa
\end{aligned}
$$

Espaços de Hilbert terão $\mathbb{C}$ como corpo base, e seu produto interno se denotará por $\langle\cdot, \cdot\rangle_{\mathfrak{H}}$, convencionandose que é antilinear no primeiro argumento e linear no segundo. Diremos que $A \in \mathcal{B}(\mathfrak{H})$ é (estritamente) positivo através da simbologia $(A>0) A \geq 0$. Ademais:

$$
\begin{aligned}
\mathcal{S}^{\perp} & =\text { complemento ortogonal de } \mathcal{S} \leq \mathfrak{H} \\
v^{*} & =\text { funcional }\langle v, \cdot\rangle_{\mathfrak{H}}, v \in \mathfrak{H} . \\
A^{*} & =\text { adjunto de } A
\end{aligned}
$$




$$
\begin{aligned}
& \sigma(A)=\text { espectro de } A \\
& \mathcal{R}(A) \quad=\quad \text { resolvente de } A \\
& \mathcal{R}(\lambda ; A)=\text { operador resolvente de } A \text { associado ao valor } \lambda \\
& \mathfrak{H}_{\lambda}(A)=\text { autoespaço de } A \text { associado ao valor } \lambda \\
& \mathrm{m}_{g}(\lambda ; A)=\text { multiplicidade geométrica de } \lambda \\
& \mathrm{m}_{a}(\lambda ; A)=\text { multiplicidade algébrica de } \lambda \\
& \mathfrak{H}^{\otimes n} \quad=\quad \mathfrak{H} \otimes{ }^{n \text { vezes }} \otimes \mathfrak{H} \\
& \otimes^{n} G=\text { tensores puros de ordem } n \text { formado com elementos de } G \subseteq \mathfrak{H} \\
& \mathfrak{D}(T) \quad=\quad \text { domínio do operador linear } T \\
& \mathscr{S}_{1}(\mathfrak{H})=\text { espaço dos operadores classe traço em } \mathfrak{H}
\end{aligned}
$$

Se $X$ e $\mathcal{C}$ são conjuntos quaisquer, $\Omega$ um aberto de $\mathbb{R}^{d}$ e $f: \Omega \longrightarrow \mathbb{R}$, resta-nos acertar:

$$
\begin{array}{ll}
\mathrm{C}^{k}(\Omega) & =\text { espaço das funções à valores reais e de classe } \mathscr{C}^{k} \text { em } \Omega, k \in \mathbb{N} \cup\{\infty\} \\
\mathrm{C}^{\omega}(\Omega) & =\text { espaço das funções reais analíticas definidas em } \Omega \\
\mathbb{T}^{n} & =[-\pi, \pi]^{n} \\
\mathbb{T}_{0}^{n} & =\text { diferencial de } f \\
\mathrm{~d} f & \text { vetor gradiente de } f \\
\vec{\nabla} f & \text { matriz hessiana de } f \\
\mathrm{Hess}(f) & =\text { suporte de funções, medidas ou distribuições em geral } \\
\text { supp } & =\text { delta de Kronecker do par }(x, y) \in \mathcal{C} \times \mathcal{C} \\
\delta_{x, y} & =\text { função delta de } x \in \mathcal{C}, \text { i.e, } \delta_{x}(y)=\delta_{x, y}, \quad y \in \mathcal{C} \\
\delta_{x} & =\text { espaço das sequências definidas em } \mathcal{C} \text { quadrado-somáveis } \\
\ell^{2}(\mathcal{C}) & \text { polinômios definidos em } \mathbb{R} \text { e com coeficientes em } \mathbb{C} \\
\mathbb{C}[t \in \mathbb{R}] & =\text { unidade imaginária } \\
\imath &
\end{array}
$$

Diremos ainda que uma sequência de funções $\left(f_{n}\right)_{n \in \mathbb{N}}$ converge pontualmente (uniformemente) para $f$ por 
meio dos símbolos $f_{n} \stackrel{p .}{\longrightarrow} f$ (respec. $f_{n} \stackrel{u .}{\longrightarrow} f$ ), ou ainda $\mathrm{s}-\lim f_{n}=f$. Para terminar, se $\mathcal{A}$ é uma álgebra involutiva, então $\mathfrak{A}^{*}(B)$ denota a subálgebra involutiva gerada por $B \subseteq \mathcal{A}$. 


\section{Introdução}

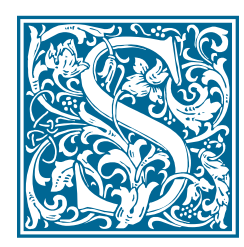

UPERCONDUTIVIDADE é uma propriedade física de certos materiais que, quando resfriados a temperaturas extremamente baixas, conduzem corrente elétrica sem resistência nem perdas perceptíveis. As aplicações tecnológicas deste fenônomeno são inúmeras: Os magnetos supercondutores são alguns dos mais poderosos eletroímãs conhecidos, e na maioria das vezes são usados em máquinas de ressonância magnética nuclear (NMR), espectrômetros de massa e até nos ímãs que direcionam o feixe em aceleradores de partículas. A grande mudança na resistência elétrica na transição do estado normal para o estado supercondutor é usada para construir os termômetros detectores de fóton em estado criogênico, e mais recentemente, eles têm sido utilizados na construção de circuitos digitais baseados na tecnologia quântica de fluxo rápido. Promissoras aplicações futuras incluem redes inteligentes de alta performance, transmissão de energia elétrica, transformadores, dispositivos de armazenamento de energia, motores elétricos, dispositivos de levitação magnética, limitadores de corrente e refrigeração magnética, etc.

Na maioria dos materiais, as propriedades supercondutoras só se revelam a temperaturas muito baixas, próximas do zero absoluto. Mas, aos poucos, os físicos têm descoberto arranjos "não convencionais", assim chamados por não obedecerem à teoria microscópica clássica de supercondutividade, conhecida pela sigla BCS e formulada nos anos 1950 pelos físicos John Bardeen, Leon Cooper e Robert Schrieffer, pela qual eles dividiram o Prêmio Nobel de 1972. Alguns desses supercondutores não convencionais são capazes de exibir essa propriedade a temperaturas mais altas. Apesar de tudo isso, as explicações teóricas à respeito deste novo estado da matéria é inconclusiva até os dias de hoje, e por isso o advento de um modelo microscópico geral para esta classe de materiais poderá revelar mecanismos que expliquem as propriedades que observamos experimentalmente.

Os artigos [BdPdSP15] e [BdSPDdP19] propõe um dos poucos e bem sucedidos modelos microscópicos relacionado aos supercondutores com alta temperatura de transição que permitem um tratamento analítico rigoroso. Neles, é feito um estudo analítico e numérico de um problema específico de três corpos formado por dois férmions (elétrons) e um bóson (bipolaron) na rede cristalina de um dado material. Para esse sistema, seus autores propõe um Hamiltoniano do tipo Hubbard que leva em conta interações bipolaronelétron, isto porque a supercondutividade em alta temperatura está possivelmente relacionada à interação atrativa de dois elétrons por intermédio de um bipolaron, que são bósons composto por um par espacial de elétrons ligados por intermédio de fônons ou mágnons.

A abordagem analítica deste Hamiltoniano $\hat{H}$ é feita através das integrais diretas de von Neumann, discutida em detalhes no capítulo 2 do presente trabalho. Heuristicamente falando, esta noção generaliza o conceito habitual de soma direta de espaços de Hilbert, atribuindo sentido a expressões do tipo

$$
\int_{X}^{\oplus} \mathfrak{H}_{x} \mathrm{~d} \mu(x)
$$

onde $\left\{\mathfrak{H}_{x}\right\}_{x \in X}$ é uma família de espaços de Hilbert indexados por um espaço de medida $(X, \mu)$. Uma classe 
especial de operadores agindo na integral acima são os ditos operadores decomponíveis: Cada um deles é obtido formalmente pela "integração direta" de uma certa família de operadores (designada por fibras)

$$
\left\{A(x) \in \mathcal{B}\left(\mathfrak{H}_{x}\right): x \in X\right\}
$$

e o produto final representado pelo símbolo

$$
\int_{X}^{\oplus} A(x) \mathrm{d} \mu(x)
$$

Um dos resultados de [BdPdSP15] que replicamos no (T.66) à frente assegura a existência de um espaço de Hilbert $\mathcal{H}$ e um espaço de medida $(X, \mu)$ onde o domínio de $\hat{H}$ é isomorfo à integral direta de $\mathcal{H}$ sobre $X$. Simbolicamente,

$$
\mathfrak{D}(\hat{H}) \simeq \int_{X}^{\oplus} \mathcal{H} \mathrm{d} \mu
$$

Um segundo teorema também nos diz que a conjugação de $\hat{H}$ com o operador unitário $U$ responsável pelo isomorfismo acima é decomponível, ou seja, existe uma família de operadores $\left\{S_{x}\right\}_{x \in X}$ agindo em $\mathcal{H}$ para o qual

$$
U \hat{H} U^{*}=\int_{X}^{\oplus} S_{x} \mathrm{~d} \mu(x)
$$

Como estamos interessados apenas nas propriedades espectrais de $\hat{H}$, não importa se extraímos elas diretamente de $\hat{H}$ ou de qualquer operador unitariamente equivalente a ele. Além disso, existem teoremas gerais que relacionam o espectro de uma integral direta de operadores com o de suas fibras - veja o (T.40) à frente. Dessa forma, troca-se o problema de investigar o espectro de $\hat{H}$ pelo problema de se analisar o espectro de uma coleção (possivelmente infinita) de operadores $S_{x}$, porém individualmente mais simples que o original.

A partir daí, diversas previsões teóricas puderam ser estabelecidas e, posteriormente, confrontadas com os dados experimentais. Em [BdSPDdP19], este modelo aplicado ao importante caso especial do cuprato supercondutor $\mathrm{La}_{2} \mathrm{CuO}_{4}$ (LaSr 214) prediz um comprimento de coerência de $21 \AA$ na dopagem ótima, sendo a mesma obtida por experimentos empíricos e pela teoria de Ginzburg-Landau. Uma questão fundamental que tem impulsionado muitos trabalhos experimentais e teóricos é a natureza da simetria (no espaço de momento) da função de onda dos pares de Cooper ${ }^{1}$, que nos cupratos supercondutores podem se manifestar nos tipos $s$-wave ${ }^{2}, d$-wave ${ }^{3}$ ou em uma mistura desses dois ${ }^{4}$. Nos supercondutores clássicos esses pares são encontrados apenas em sua fase supercondutora (à temperaturas $<T_{c}$ ), enquanto que nos supercondutores não-convencionais eles são detectados (com simetria $d$-wave no caso dos cupratos) desde o regime de "pseudogap", ou seja, a temperaturas muito mais elevadas que a temperatura de transição para a fase supercondutora. Embora este fenômeno tenha sido explicado com sucesso pela teoria BCS no caso clássico,

\footnotetext{
${ }^{1}$ Um par de Cooper é o nome dado a elétrons em um metal que estão ligados de uma certa maneira descrita pela primeira vez por Leon Cooper. De forma simplificada, um elétron é repelido dos outros elétrons devido a sua carga similar, mas isto também atrai os íons positivos de forma que estes íons atraem a outros elétrons (a interação elétron-fônon). Esta atração devida aos íons deslocados pode superar a repulsão dos elétrons tendo a mesma carga, causando seu emparelhamento. Embora o processo de formação de um bipolaron também seja causado pela combinação de pares de elétrons e deformações na rede subjacente, vale lembrar que estes são altamente massivos e localizados, enquantos os pares de Cooper são extensos e leves.

${ }^{2} \mathrm{~A}$ função de onda dos pares é invariante por rotações de $90^{\circ}$.

${ }^{3} \mathrm{~A}$ função de onda dos pares é antisimétrica com respeito à rotações de $90^{\circ}$.

${ }^{4}$ Simetrias do tipo $p$-wave (em que a função de onda é antisimétrica com respeito à rotações de $180^{\circ}$ ) nunca foram observadas em cupratos supercondutores.
} 
o caso não convencional deixa de ser uma incógnita para a comunidade científica a partir dos trabalhos recentes [BdPdSP15] e [BdSPDdP19], onde o modelo ajustado com os parâmetros prototípicos do LaSr 214 prediz a formação de pares $d$-wave inclusive na região de "pseudogap". Outra previsão teórica do modelo concomitante com os dados empíricos conhecidos inclui a temperatura de pseudogap $T^{*}$ deste material na dopagem ótima, estimada em $400 K$. Também não podemos deixar de mencionar [ $\left.\mathrm{P}^{+} 18\right]$, onde o modelo foi usado para se obter critérios que asseguram a formação de ondas de densidade nos estados de equilíbrio, que é uma outra propriedade conhecida de cupratos supercondutores.

A partir das evidências listadas no parágrafo acima, nos convencemos de que o modelo proposto nestes artigos captura de forma adequada a física por trás do problema, e nesse mesmo espírito que o utilizamos no presente trabalho para:

1) Estudar o aparecimento de alguns canais de espalhamento para o sistema em questão (capítulo 6).

2) Investigar a existência de um limite hard-core bem comportado, relevante na simplificação do problema de muitos corpos (capítulo 5). Essa busca se motiva em estudos numéricos realizados em um dos artigos base, onde se detectou a presença de um comportamento efetivo da repulsão eletrônica no regime de hard-core, ou seja, em certo ponto o modelo satura de modo a não perceber diferenças concretas entre repulsões com magnitudes suficientemente grandes. Além disso, a repulsão eletrônica nos cupratos se encontra na situação descrita anteriormente pois é extremamente forte e localizada, sendo neste regime que o modelo confirma que as propriedades observadas nesses supercondutores de fato ocorrem, em particular o pareamento $d$-wave.

3) Fornecer critérios que asseguram a existência de uma relação de dispersão que dá origem a pares ligado com baixa energia (típicamente $d$-wave), além de fórmulas explícitas para o cálculo da velocidade de grupo e tensor de massa do sistema neste regime (seção 4.4).

4) Estender alguns resultados teóricos obtidos nesses artigos base. (seção 4.3).

Existem muitas outras classes de modelos microscópicos que se propõe a explicar a supercondutividade com alta temperatura de transição. A escola mais famosa (por exemplo) atribui esse fenômeno as propriedades antiferromagnéticas exibida por esses supercondutores não-convencionais. Embora esse tipo de modelo explique de maneira relativamente simples a presença de uma simetria $d$-wave na função de onda dos pares, eles não conseguem encontrar uma transição de fase para estes materiais, ou seja, o modelo não prevê a existência de uma fase supercondutora no final das contas. Em contrapartida, a escola bipolarônica (que relaciona o mecanismo de supercondução com alta temperatura ao bipolaron) considerada nos artigos base foi bem sucedida em identificar transições de fase em seus trabalhos anteriores, enquanto que o pareamento $d$-wave só pode ser explicado de maneira indireta. Por muito tempo essa classe de modelos também enfrentou as questões levantadas pelo paradoxo da massa ${ }^{5}$, ao ponto em que alguns autores chegaram a propor "bipolarons leves" em seus modelos bipolarônicos, mesmo sabendo que este tipo de partícula nunca foi observado. Embora o debate à respeito do mecanismo responsável pela supercondutividade em alta temperatura esteja fortemente polarizado entre essas duas escolas, existem outras menos populares como a escola do modelo de Hubbard que associa esse fenômeno a um efeito puramente cinético entre os elétrons dentro do cristal.

Embora ainda não exista uma teoria microscópica convincente para a supercondutividade em alta temperatura, vemos que os artigos base propõe um modelo bastante relevante que explica várias propriedades

\footnotetext{
${ }^{5}$ Apesar das inúmeras evidências empíricas favoráveis a correlação entre bipolaron e supercondutividade em alta temperatura, sua massa é muito maior que a massa observada dos portadores de cargas, que também são pares. Felizmente, este problema foi resolvido em [BdSPDdP19].
} 
destes supercondutores (pareamento $d$-wave, massa efetiva dos pares, temperatura de pseudogap, etc), não apresenta patologias evidentes e tem o bom gosto de poder ser analisado de modo rigoroso por métodos bem estabelecidos em análise funcional. Como dito anteriormente, nesta dissertação vamos estender alguns resultados rigorosos previstos nesse modelo e provar coisas novas (canais de espalhamento, limite de hard-core) com base no mesmo.

\section{Organização da dissertação}

O corpo principal do texto foi dividido em duas partes. Na primeira delas, apresenta-se as principais ferramentas matemáticas que serão utilizadas para se examinar as fibras $S_{x}$, de maneira que, ao menos do ponto de vista matemático, este texto possa ser considerado autocontido. Além disso, algumas demonstrações, apesar de não serem demasiadamente complicadas, não parecem ser facilmente encontradas na literatura e, portanto, encontram-se redigidas aqui.

- Na seção 1.1, apresentamos um tipo de cálculo funcional boreliano mais adequado para lidar com famílias de operadores. Nesta mesma seção, também introduzimos o importante conceito de espaço espectral absolutamente contínuo, crucial para a discussão que virá na seção 3.2.

- O critério de Weyl mostrou-se uma ferramente extremamente útil para se investigar a parte essencial do espectro de cada uma das fibras $S_{x}$. Em vista disso, a seção 1.2 dedica-se única e exclusivamente a ele.

- A seção 1.3 é devotada ao ilustre princípio de Birman-Schwinger, que nos fornece meios eficientes de se identificar autovalores para as fibras $S_{x}$.

- No capítulo 2, apresentamos os elementos principais da teoria das integrais diretas de von Neumann.

- Na seção 3.1, discutimos o formalismo moderno da segunda quantização, que é base para a formulação do Hamiltoniano $\hat{H}$.

- Na seção 3.2, apresentamos alguns rudimentos da teoria abstrata de espalhamento, que mais tarde virá a ser aplicado no capítulo 6.

$\mathrm{Na}$ segunda parte, inaugura-se efetivamente o estudo do modelo microscópico para supercondutores não convencionais proposto em [BdPdSP15]. Para o conforto do leitor, este modelo é reintroduzido nas seções iniciais 4.1 e 4.2. De fato:

- Na seção 4.1, resolvemos questões de domínio, limitação e boa definição de $\hat{H}$.

- Na seção 4.2, damos rigor à decomposição em fibras de $\hat{H}$, motivada informalmente agora há pouco.

\section{Principais Resultados}

Segue uma lista comentada dos principais resultados obtidos nesta dissertação. Afim de evitar certas tecnicalidades logo na introdução, alguns deles não se encontram enunciados da maneira em que aparecem no texto.

Teorema. (Bru-Pedra-Pasquale, [BdPdSP15]) Denotando $\varrho \equiv(2 \pi)^{-2}(\mathfrak{m} \otimes \mathfrak{m})$, tem-se

$$
\mathfrak{D}(\hat{H}) \simeq_{U} \int_{\mathbb{T}^{2}}^{\oplus} \mathrm{L}^{2}\left(\mathbb{T}^{2}\right) \oplus \mathbb{C} \mathrm{d} \varrho
$$


Além disso, a conjugação de $\hat{H}$ pelo operador unitário $U$ é decomponível pela integral direta de $\mathrm{L}^{2}\left(\mathbb{T}^{2}\right) \oplus \mathbb{C}$, valendo

$$
U \hat{H} U^{*}=\int_{\mathbb{T}^{2}}^{\oplus}\left(\begin{array}{ll}
\Xi_{1,1}(k) & \Xi_{1,2}(k) \\
\Xi_{2,1}(k) & \Xi_{2,2}(k)
\end{array}\right) \mathrm{d} \varrho(k)
$$

onde

$$
\begin{array}{rll}
\Xi_{1,1}: \mathbb{T}^{2} \longrightarrow \mathcal{B}\left(\mathrm{L}^{2}\left(\mathbb{T}^{2}\right)\right) & ; & \Xi_{1,1}(k) \varphi \doteq \mathrm{M}_{\mathfrak{f}(k)} \varphi+\sum_{z \in \mathbb{Z}^{2}} \mathrm{U}(z) \mathrm{P}_{z} \varphi \\
\Xi_{1,2}: \mathbb{T}^{2} \longrightarrow \mathcal{B}\left(\mathbb{C}, \mathrm{L}^{2}\left(\mathbb{T}^{2}\right)\right) & ; & \Xi_{1,2}(k) z \doteq \hat{v}(k) z \mathfrak{d}(k) \\
\Xi_{2,1}: \mathbb{T}^{2} \longrightarrow \mathrm{L}^{2}\left(\mathbb{T}^{2}\right)^{*} & ; & \Xi_{2,1}(k) \varphi \doteq \hat{v}(k)\langle\mathfrak{d}(k), \varphi\rangle_{2} \\
\Xi_{2,2}: \mathbb{T}^{2} \longrightarrow \mathcal{B}(\mathbb{C}) & ; & \Xi_{2,2}(k) z \doteq \mathfrak{b}(k) z
\end{array}
$$

sendo que $\mathrm{P}_{z}$ denota o projetor ortogonal de $\mathrm{L}^{2}\left(\mathbb{T}^{2}\right)$ no subespaço fechado

$$
\mathbb{C} e^{-\imath\langle\cdot, z\rangle}, \quad z \in \mathbb{Z}^{2}
$$

e $\mathfrak{d}, \mathfrak{f}: \mathbb{T}^{2} \longrightarrow \mathrm{C}\left(\mathbb{T}^{2}\right)$ e $\mathfrak{b} \in \mathrm{C}\left(\mathbb{T}^{2}\right)$ são definidos por

$$
\begin{gathered}
\mathfrak{b}(k) \doteq \epsilon h_{b}\{2-\cos (k)\} \quad, \quad \mathfrak{f}(k)(h) \doteq \epsilon\{4-\cos (h-k)-\cos (h)\} \\
\mathfrak{d}(k)(h) \doteq 2\{\cos (h-k)+\cos (2 h-k)+1\}
\end{gathered}
$$

com a convenção de que

$$
\cos (k) \equiv \cos \left(k_{1}\right)+\cos \left(k_{2}\right), \quad k=\left(k_{1}, k_{2}\right) \in \mathbb{T}^{2}
$$

Dado que $U$ é definido por meio de transformadas de Fourier, e estas são responsáveis pela dualidade entre espaço de posição e momento, no decorrer do texto se referimos a variável de fibra $k$ por "momento".

Teorema. Seja $k \in \mathbb{T}_{0}^{2}$ e $h_{b} \in\left[0, \frac{1}{2}\right]$. São verdadeiras as seguintes afirmações:

a) Existe um, e apenas um autovalor $\mathrm{E}(k)$ de $\Xi(k)$ estritamente menor que $4 \epsilon-2 \epsilon \cos (k / 2)$. Neste caso,

$$
\begin{cases}\mathrm{E}(k)<\mathfrak{b}(k) & , \hat{v}(k) \neq 0 \\ \mathrm{E}(k)=\mathfrak{b}(k) & , \hat{v}(k)=0\end{cases}
$$

b) Assim definida, E tem mesmo grau de regularidade que $\hat{v}$ no interior de $\mathbb{T}_{0}^{2}$.

Este teorema nos fornece critérios para o qual a aplicação E define uma relação de dispersão conforme a (D.73). No presente trabalho, denominamo-o como "relação de dispersão de baixa energia" pois interpretase o espectro da fibra $\Xi(k)$ como os valores energéticos disponíveis ao sistema quando o mesmo se encontra com momento não nulo $k$, sendo que $\mathrm{E}(k)=\min \sigma(\Xi(k))$.

Teorema. Seja $h_{b} \in\left[0, \frac{1}{2}\right]$. São verdadeiras as seguintes asserções:

a) Para todo $k \in \mathbb{T}_{0}^{2}$, tem-se

$$
\mathrm{E}^{(\infty)}(k) \doteq \lim _{U_{0} \rightarrow \infty} \mathrm{E}\left(U_{0}, k\right)=\sup _{U_{0} \geq 0} \mathrm{E}\left(U_{0}, k\right)
$$

b) Igualmente a função E, verifica-se

$$
\begin{cases}\mathrm{E}^{(\infty)}(k)<\mathfrak{b}(k) & , \hat{v}(k) \neq 0 \\ \mathrm{E}^{(\infty)}(k)=\mathfrak{b}(k) & , \hat{v}(k)=0\end{cases}
$$

c) Se $\hat{v}$ é de classe $\mathscr{C}^{d \geq 1}$ no interior de $\mathbb{T}_{0}^{2}, \mathrm{E}^{(\infty)}$ também será. 
Este resultado se enquadra ao contexto de repulsões eletrônicas com alcançe zero ${ }^{6}$. Ele comprova a existência de uma relação de dispersão efetiva associada à E conforme a (D.81), e estabelece, portanto, que o comportamento efetivo no regime de hard-core da repulsão eletrônica (visto inicialmente por meios numéricos em um dos artigos base) está previsto no modelo.

\section{Teorema.}

$$
W^{ \pm}\left(\int_{\mathbb{T}^{2}}^{\oplus} \Xi(k) \mathrm{d} \varrho(k), \int_{\mathbb{T}^{2}}^{\oplus} \mathrm{M}_{\mathfrak{f}(k)} \mathrm{d} \varrho(k) ; J\right) J^{*}=\int_{\mathbb{T}^{2}}^{\oplus} \Omega^{ \pm}\left(\Xi(k), \mathrm{M}_{\mathfrak{f}(k)} \oplus \Xi_{2,2}(k)\right) \mathrm{d} \varrho(k)
$$

e se $h_{b} \in\left[0, \frac{1}{2}\right]$, também vale

$$
W^{ \pm}\left(\int_{\mathbb{T}^{2}}^{\oplus} \Xi(k) \mathrm{d} \varrho(k), \int_{\mathbb{T}^{2}}^{\oplus} \mathrm{E}(k) \mathrm{d} \varrho(k) ; \mathbf{A}\right)=\mathbf{A} P_{\mathrm{ac}}\left(\int_{\mathbb{T}^{2}}^{\oplus} \mathrm{E}(k) \mathrm{d} \varrho(k)\right)
$$

Aqui se estabelece a existência de dois canais de espalhamento para o sistema em consideração. Um deles afirma que para longos tempos o par de Cooper se desfaz de modo que os elétrons resultantes se difundem livremente na rede do cristal, enquanto o outro diz que o emparelhamento permanece íntegro (durante todo instante de tempo) e se propaga como uma partícula livre com dispersão E.

Teorema. Seja $h_{b} \in\left[0, \frac{1}{2}\right]$. Então

$$
\mathrm{s}-\lim _{U_{0} \rightarrow \infty} \exp \left(-\imath t \int_{\mathbb{T}^{2}}^{\oplus} \Xi\left(U_{0}, k\right) \mathrm{d} \varrho(k)\right) \mathbf{A}\left(U_{0}\right)=\mathbf{A}^{(\infty)}\left(\int_{\mathbb{T}^{2}}^{\oplus} e^{-\imath t \mathrm{E}^{(\infty)}(k)} \mathrm{d} \varrho(k)\right)
$$

e se além disso $\hat{v}$ for real analítica no interior de seu domínio, também vale que

$$
\text { s- } \lim _{U_{0} \rightarrow \infty} W^{ \pm}\left(\int_{\mathbb{T}^{2}}^{\oplus} \Xi\left(U_{0}, k\right) \mathrm{d} \varrho(k), \int_{\mathbb{T}^{2}}^{\oplus} \mathrm{E}\left(U_{0}, k\right) \mathrm{d} \varrho(k) ; \mathbf{A}\left(U_{0}\right)\right)=\mathbf{A}^{(\infty)}
$$

Este resultado reuni os dois principais tópicos abordados nesta dissertação (limite hard-core e canais de espalhamento). A primeira parte dá sentido a dinâmica do sistema no limite de hard-core como sendo a dinâmica de uma partícula efetiva com dispersão $\mathrm{E}^{(\infty)}$, e a segunda parte prevê sob certas hipóteses a estabilidade do canal de pareamento $d$-wave com relação ao limite de hard-core.

São Paulo, Junho de 2020

Alan Ramer dos Santos

\footnotetext{
${ }^{6}$ Veja a (D.78) à frente.
} 
Todas as opiniões que há sobre a Natureza

Nunca fizeram crescer uma erva ou nascer uma flor.

Toda a sabedoria a respeito das cousas

Nunca foi cousa em que pudesse pegar como nas cousas;

Se a ciência quer ser verdadeira,

Que ciência mais verdadeira que a das cousas sem ciência?

Fecho os olhos e a terra dura sobre que me deito

Tem uma realidade tão real que até as minhas costas a sentem.

Não preciso de raciocínio onde tenho espáduas.

Alberto Caeiro, Heterônimo de Fernando Pessoa (1888-1935)

"Todas as Opiniões que Há sobre a Natureza" (Poemas Inconjuntos) 



\section{Parte I}

\section{Fundamentos Matemáticos}





\section{Capítulo 1}

\section{Tópicos em Análise Espectral}

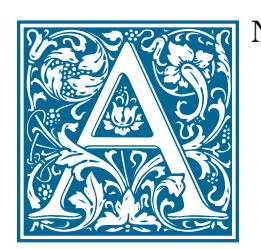

NÁLISE ESPECTRAL é o nome que se atribui ao ramo da análise funcional que se dedica a generalização da teoria de autovalores e autovetores de uma matrix quadrada para um quadro conceitual muito mais amplo de espaços e operadores. Apesar de todo interesse matemático que pairava sobre este seguimento, mais tarde ele viria explicar inúmeros fenômenos quânticos, como o espectro de emissão dos elementos químicos. Teve como um de seus precursores o brilhante matemático germânico David Hilbert ${ }^{1}$, que vislumbrado com as façanhas de sua teoria, disse o seguinte: "I developed my theory of infinitely many variables from purely mathematical interests, and even called it 'spectral analysis' without any presentiment that it would later find application to the actual spectrum of physics".

Noções Gerais e Teoremas: Medidas Espectrais, Fundo e Teto do Espectro de um Operador Autodajunto, Lema da Classe Monótona Funcional, Topologia da Convergência Compacta, Cálculo Funcional Boreliano, Projetor Espectral, Espaço Espectral Absolutamente Contínuo, Convergência Fraca, Teorema de Banach-Alaoglu, Sequência de Weyl, Espectro de Weyl, Critério de Weyl, Perturbação do Espectro Essencial por um Operador Compacto, Operador e Teorema de Birman-Schwinger.

\subsection{As Medidas Espectrais}

"A Matemática é a única linguagem que temos em comum com a natureza."

Stephen Hawking ${ }^{2}$

Partindo do pressuposto que o leitor tenha familiaridade com o cálculo funcional contínuo de operadores autoadjuntos e limitados, o resto fica por nossa conta.

Teorema 1. Seja $\mathfrak{H}$ um espaço de Hilbert e $A \in \mathcal{B}(\mathfrak{H})$ autoadjunto. Então, para cada $\psi \in \mathfrak{H}$, existe uma única medida de Radon $\Omega_{\psi}$ no espectro de $A$ que verifica a relação

$$
\langle\psi, f(A) \psi\rangle_{\mathfrak{H}}=\int_{\sigma(A)} f(\lambda) \mathrm{d} \Omega_{\psi}(\lambda)
$$

para toda e qualquer $f \in \mathrm{C}(\sigma(A))$.

\footnotetext{
${ }^{1}$ David Hilbert, $\odot(23 / 01 / 1862)-\dagger(14 / 02 / 1943)$

${ }^{2}$ Stephen William Hawking, $\odot(08 / 01 / 1942)-\dagger(14 / 03 / 2018)$
} 
Demonstração. Basta aplicar o teorema de representação de Riesz-Markov-Kakutani no funcional linear

$$
\mathrm{C}_{c}(\sigma(A)) \stackrel{3}{=} \mathrm{C}(\sigma(A)) \ni f \longmapsto\langle\psi, f(A) \psi\rangle_{\mathfrak{H}} \in \mathbb{C}
$$

positivo em função das implicações

$$
f \geq 0 \Longrightarrow \sigma(f(A))=f(\sigma(A)) \subseteq[0,+\infty) \stackrel{\text { def. }}{\Longrightarrow} f(A) \geq 0 \Longrightarrow\langle\psi, f(A) \psi\rangle_{\mathfrak{H}} \stackrel{4}{=}\|R \psi\|_{\mathfrak{H}}^{2} \geq 0
$$

e limitado por conta de

$$
\left|\langle\psi, f(A) \psi\rangle_{\mathfrak{H}}\right| \leq\|f(A)\|_{\text {op }}|| \psi\left\|_{\mathfrak{H}}^{2}=\right\| \psi\left\|_{\mathfrak{H}}^{2}\right\| f \|_{\infty}
$$

seja qual for $f \in \mathrm{C}(\sigma(A))$.

Definição 2 (Medida Espectral). Com a notação e hipóteses do teorema acima, $\Omega_{\psi}$ é dita ser a medida espectral de A subordinada ao vetor $\psi \in \mathfrak{H}$.

Este conceito é de suma importância em análise espectral porque nos disponibiliza de todas as ferramentas matemáticas contempladas na teoria de medida e integração para se estudar as propriedades espectrais de operadores.

Proposição 3. Se $\mathfrak{H}$ é um espaço de Hilbert e $A \in \mathcal{B}(\mathfrak{H})$ autoadjunto, então

$$
\min (\sigma(A))=\inf _{\psi \in \mathbb{S}^{1}(\mathfrak{H})}\langle\psi, A \psi\rangle_{\mathfrak{H}}
$$

além do que

$$
\max (\sigma(A))=\sup _{\psi \in \mathbb{S}^{1}(\mathfrak{H})}\langle\psi, A \psi\rangle_{\mathfrak{H}}
$$

Demonstração. Para todo $\psi \in \mathbb{S}^{1}(\mathfrak{H})$, tem-se

$$
\begin{aligned}
\langle\psi, A \psi\rangle_{\mathfrak{H}}=\int_{\sigma(A)} \lambda \mathrm{d} \Omega_{\psi}(\lambda) \geq\left(\min _{\lambda \in \sigma(A)} \lambda\right) \int_{\sigma(A)} 1 \mathrm{~d} \Omega_{\psi}=\min (\sigma(A))\langle\psi, \mathbb{1} \psi\rangle_{\mathfrak{H}} & = \\
& =\min (\sigma(A))\|\psi\|_{\mathfrak{H}}^{2}=\min (\sigma(A))
\end{aligned}
$$

e $\log 0$

$$
\min (\sigma(A)) \leq \inf _{\psi \in \mathbb{S}^{1}(\mathfrak{H})}\langle\psi, A \psi\rangle_{\mathfrak{H}} \doteq L
$$

Em contra partida, $\lambda<L$ implica

$$
0<L-\lambda \leq\langle\psi, A \psi\rangle_{\mathfrak{H}}-\lambda=\langle\psi, A \psi\rangle_{\mathfrak{H}}-\lambda\langle\psi, \psi\rangle_{\mathfrak{H}}=\langle\psi,(A-\lambda \mathbb{1}) \psi\rangle_{\mathfrak{H}} \leq\|(A-\lambda \mathbb{1}) \psi\|_{\mathfrak{H}} \quad \forall \psi \in \mathbb{S}^{1}(\mathfrak{H})
$$

o que torna $A-\lambda \mathbb{1}$ é inversível ${ }^{5}$, e $\operatorname{logo} \lambda \notin \sigma(A)$. Constatado a ausência de pontos espectrais de $A$ menores que $L$, segue

\footnotetext{
${ }^{3} \mathrm{O}$ espectro de todo elemento de uma álgebra de Banach com unidade é compacto.

${ }^{4}$ Como todo operador positivo agindo em $\mathfrak{H}$ admite uma raiz quadrada, $f(A)=R^{2}$ para algum $R \in \mathcal{B}(\mathfrak{H})$.

${ }^{5}$ Confira o apêndice A.
} 


$$
L \leq \min (\sigma(A))
$$

e a segunda asserção é análoga.

Corolário 4. Seja $\mathfrak{H}$ um espaço de Hilbert e $A, B \in \mathcal{B}(\mathfrak{H})$ autoadjuntos. As seguintes afirmações são verdadeiras:
a) $A \geq 0 \Longleftrightarrow\langle\psi, A \psi\rangle_{\mathfrak{H}} \geq 0 \quad \forall \psi \in \mathfrak{H}$
b) $A \leq B \Longrightarrow \min (\sigma(A)) \leq \min (\sigma(B)), \max (\sigma(A)) \leq \max (\sigma(B))$.

Demonstração. De fato,

$$
A \geq 0 \Longleftrightarrow \sigma(A) \subseteq[0,+\infty) \Longleftrightarrow \min (\sigma(A)) \geq 0 \Longleftrightarrow\langle\psi, A \psi\rangle_{\mathfrak{H}} \geq 0 \quad \forall \psi \in \mathbb{S}^{1}(\mathfrak{H})
$$

prova a), e nos permite aplicá-lo em $B-A \geq 0$, resultando

$$
0 \leq\langle\psi,(B-A) \psi\rangle_{\mathfrak{H}}=\langle\psi, B \psi\rangle_{\mathfrak{H}}-\langle\psi, A \psi\rangle_{\mathfrak{H}} \quad \forall \psi \in \mathbb{S}^{1}(\mathfrak{H})
$$

ou seja,

$$
\langle\psi, A \psi\rangle_{\mathfrak{H}} \leq\langle\psi, B \psi\rangle_{\mathfrak{H}} \quad \forall \psi \in \mathbb{S}^{1}(\mathfrak{H})
$$

e portanto b).

Dado que nas demais aplicações seja conveniente que $\Omega_{\psi}$ esteja definida nos borelianos de $\mathbb{R}$, rebatizamos a medida espectral de $A$ subordinada ao vetor $\psi$ por

$$
\hat{\Omega}_{\psi}(B) \doteq \Omega_{\psi}(B \cap \sigma(A)), \quad B \in \mathfrak{B}_{\mathbb{R}}
$$

Note que a finitude de $\hat{\Omega}_{\psi}$ junto a compacidade de $\sigma(A)$ torna toda $g \in \mathfrak{B}_{\mathrm{c}}(\mathbb{R}) \hat{\Omega}_{\psi}$-integrável, valendo

$$
\int_{\mathbb{R}} g(\lambda) \mathrm{d} \hat{\Omega}_{\psi}(\lambda)=\int_{\sigma(A)} g(\lambda) \mathrm{d} \Omega_{\psi}(\lambda)
$$

Por fim, voltamos a denotar $\hat{\Omega}_{\psi}$ sem o uso do chapéu.

Nosso próximo grande objetivo é dar sentido a expressões do tipo $g(A)$, em que $g \in \mathfrak{B}_{\mathrm{c}}(\mathbb{R})$. Para tal, exporemos um fato técnico do qual muitas das construções que faremos daqui pra frente se beneficiam:

Teorema 5 (Lema da Classe Monótona Funcional). Seja $\mathcal{M} \subseteq \mathfrak{B}_{\mathrm{c}}(\mathbb{R})$ tal que:

a) $S e\left(g_{n}\right)_{n \in \mathbb{N}}$ é uma sequência de funções em $\mathcal{M}$ uniformemente limitada nos compactos de $\mathbb{R} e$ tal que $g_{n} \stackrel{p .}{\longrightarrow} g$, então $g \in \mathcal{M}$.

b) $\mathrm{C}(\mathbb{R}) \subseteq \mathcal{M}$

Então, vale $\mathcal{M}=\mathfrak{B}_{\mathrm{c}}(\mathbb{R})$. Noutras palavras, nenhum subconjunto próprio de $\mathfrak{B}_{\mathrm{c}}(\mathbb{R})$ cumpre ambas as sentenças acima.

Demonstração. Seja $\mathfrak{S}$ a família formada pelos subconjuntos de $\mathfrak{B}_{\mathrm{c}}(\mathbb{R})$ que cumpre as exigências estipuladas no enunciado. Lembrando que funções contínuas são limitadas nos compactos em conformidade 
com o teorema de Weierstrass ${ }^{6}$, tal coleção se vê não vazia por que contém o próprio $\mathfrak{B}_{\mathrm{c}}(\mathbb{R})$ como um de seus elementos. Eleita uma subcoleção $\mathfrak{C} \subseteq \mathfrak{S}$ qualquer, ponha

$$
X \doteq \bigcap\{Y: Y \in \mathcal{C}\}
$$

Por hipótese

$$
\mathrm{C}(\mathbb{R}) \subseteq Y \quad \forall Y \in \mathcal{C} \Longrightarrow \mathrm{C}(\mathbb{R}) \subseteq X
$$

e se dada $f$, limite pontual de uma sequência de funções em $X$ (e portanto em cada $Y \in \mathcal{C}$ ) uniformemente limitada nos compactos, teremos

$$
f \in Y \quad \forall Y \in \mathcal{C} \Longrightarrow f \in X
$$

e em suma $X \in \mathfrak{S}$. Constatado que a família $\mathfrak{S}$ é fechada por intersecções, o próximo passo trata-se de avaliar se

$$
\mathcal{A} \doteq \bigcap\{R: R \in \mathfrak{S}\} \in \mathfrak{S}
$$

constitui uma álgebra de funções. De fato, sob o auxílio de alguma $f_{0} \in \mathrm{C}(\mathbb{R})$, ponha

$$
\mathcal{A}_{f_{0}} \doteq\left\{g \in \mathfrak{B}_{\mathrm{c}}(\mathbb{R}): g+f_{0} \in \mathcal{A}\right\}
$$

Tal conjunto contém as contínuas por que dada $g \in \mathrm{C}(\mathbb{R})$, efetua-se

$$
\begin{aligned}
& \mathfrak{B}_{\mathrm{c}}(\mathbb{R}) \in \mathfrak{S} \Longrightarrow \mathrm{C}(\mathbb{R}) \subseteq \mathfrak{B}_{\mathrm{c}}(\mathbb{R}) \Longrightarrow g \in \mathfrak{B}_{\mathrm{c}}(\mathbb{R})
\end{aligned}
$$

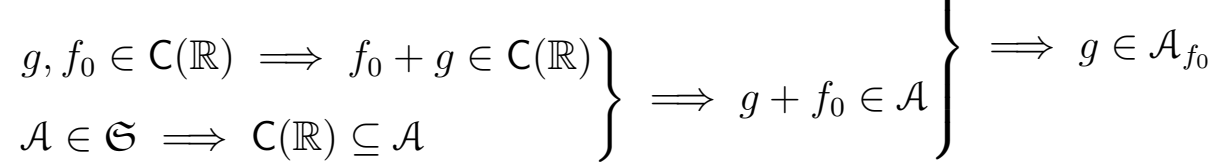

Aliás, se $\left(h_{n}\right)_{n \in \mathbb{N}}$ é uma sequência de funções em $\mathcal{A}_{f_{0}}$ uniformemente limitada nos compactos e convergindo pontualmente para $h$, também vale

$$
\left.\begin{array}{l}
\left.\begin{array}{l}
\mathcal{A}_{f_{0}} \subseteq \mathfrak{B}(\mathbb{R}) \Longrightarrow h_{n} \in \mathfrak{B}(\mathbb{R}) \\
h_{n} \stackrel{p .}{\longrightarrow} h
\end{array}\right\} \Longrightarrow h \in \mathfrak{B}(\mathbb{R}) \\
\left.\begin{array}{l}
h_{n} \stackrel{p .}{\longrightarrow} h \\
\sup _{n, K}\left|h_{n}\right|<+\infty \quad \forall K \subseteq \mathbb{R} \text { cpt. }
\end{array}\right\} \Longrightarrow \sup _{K}|h|<+\infty \quad \forall K \subseteq \mathbb{R} \text { cpt. }
\end{array}\right\} \Longrightarrow h \in \mathfrak{B}_{\mathrm{c}}(\mathbb{R})
$$

\footnotetext{
${ }^{6}$ Karl Wilhelm Theodor Weierstraß, $\odot(31 / 10 / 1815)-\dagger(19 / 02 / 1897)$
} 
e portanto $h \in \mathcal{A}_{f_{0}}$, ou seja, $\mathcal{A}_{f_{0}}$ se apresenta como um elemento de $\mathfrak{S}$, e como qualquer outro precisa conter $\mathcal{A}$. Em atenção a arbitrariedade na escolha de $f_{0}$, concluí-se

$$
f \in \mathrm{C}(\mathbb{R}), g \in \mathcal{A}\left(\subseteq \mathcal{A}_{f}\right) \Longrightarrow f+g \in \mathcal{A}
$$

e agora retomamos o argumento com uma nova função auxiliar $g_{0} \in \mathcal{A}$. De fato,

$$
\mathcal{A}_{g_{0}} \doteq\left\{g \in \mathfrak{B}_{\mathrm{c}}(\mathbb{R}): g+g_{0} \in \mathcal{A}\right\}
$$

contém $C(\mathbb{R})$ pelo o que acabamos de provar, e se trocamos $f_{0}$ por $g_{0}$ no segundo esquemograma ${ }^{7}$, vê-se que a condição a) também se cumpre. $\operatorname{Logo} \mathcal{A}_{g_{0}} \in \mathfrak{S}$, portanto $\mathcal{A} \subseteq \mathcal{A}_{g_{0}}$, e sendo $g_{0}$ arbitrário

$$
f \in \mathcal{A}, g \in \mathcal{A}\left(\subseteq \mathcal{A}_{f}\right) \Longrightarrow f+g \in \mathcal{A}
$$

O modelo argumentativo está lançado, e cabe ao leitor reproduzí-lo para o produto pontual de funções ${ }^{8}$. Concluído mais um objetivo, a próxima tarefa consiste em mostrar que

$$
\Delta \doteq\left\{G \in \mathfrak{B}_{\mathbb{R}}: \chi_{G} \in \mathcal{A}\right\}
$$

contempla todos os borelianos de $\mathbb{R}$. Para tal, basta mostrar que $\Delta$ define uma $\sigma$-álgebra de subconjuntos de $\mathbb{R}$ contendo todo e qualquer aberto do mesmo. De fato,

- Se $E, F \in \Delta$ são tais que $E \subseteq F$, então $\chi_{F \backslash E}=\chi_{F}-\chi_{E} \in \mathcal{A} \Longrightarrow F \backslash E \in \Delta$

- Se $E, F \in \Delta$, então $\chi_{E \cap F}=\chi_{E} \cdot \chi_{F} \in \mathcal{A} \Longrightarrow E \cap F \in \Delta$

- Se $\left\{E_{n}\right\}_{n \in \mathbb{N}} \subseteq \Delta$ são tais que $E_{k} \cap E_{l}=\varnothing$ quando $k \neq l$, então

$$
\left.\begin{array}{c}
\sum_{k=1}^{n} \chi_{E_{k}} \in \mathcal{A} \\
\sum_{k=1}^{n} \chi_{E_{k}} \stackrel{p .}{\longrightarrow} \chi_{\bigcup_{1}^{\infty} E_{n}} \\
\sum_{k=1}^{n} \chi_{E_{k}} \leq 1
\end{array}\right\} \Longrightarrow \chi_{\cup_{1}^{\infty} E_{n}} \in \mathcal{A} \Longrightarrow \bigcup\left\{E_{n}: n \in \mathbb{N}\right\} \in \Delta
$$

o que em palavras quer dizer que $\Delta$ é fechado por diferença própria, intersecção finita, reunião enumerável disjunta, e além disso, contém $\mathbb{R}$. Pois é um fato que tais propriedades configuram uma definição alternativa de $\sigma$-álgebra. Na sequência, seja $F \subseteq \mathbb{R}$ um fechado e ponha

$$
u_{n}(t) \doteq 2^{-\mathrm{d}(t, F) n}, \quad t \in \mathbb{R}
$$

Note que toda $u_{n} \in \mathcal{A}$ por que é contínua, além do que $u_{n} \leq 1$. Pra completar,

$$
t \in F \Longrightarrow \mathrm{d}(t, F)=0 \Longrightarrow u_{n}(t)=1 \Longrightarrow \lim _{n} u_{n}(t)=1=\chi_{F}(t)
$$

e por causa que $F$ é fechado,

$$
t \notin F \Longrightarrow \mathrm{d}(t, F)>0 \Longrightarrow \lim _{n} u_{n}(t)=0=\chi_{F}(t)
$$

\footnotetext{
${ }^{7}$ Salvo a parte enfática " $f_{0} \in \mathrm{C}(\mathbb{R}) \Longrightarrow$ ", que simplesmente desconsideramos.

${ }^{8} \mathrm{~A}$ mutiplicação por escalar é consequência pois pode ser vista como produto em que um dos fatores é uma função constante.
} 
De qualquer forma $u_{n} \stackrel{p .}{\longrightarrow} \chi_{F}$, e que por $\mathcal{A} \in \mathfrak{S}$, implica $\chi_{F} \in \mathcal{A}$, e $\operatorname{logo} F \in \Delta$. Por complementação, uma $\sigma$-álgebra que contém todo fechado também contém todo aberto, impreterivelmente.

Finalmente estamos em posição de concluir a demonstração. Efetivamente, $\mathcal{A}$ contém todas as funções simples por que é um espaço vetorial que abriga as funções características de borelianos de $\mathbb{R}$. Em adição, sabe-se que toda função boreliana, em particular aquelas que se encontram em $\mathfrak{B}_{\mathrm{c}}(\mathbb{R})$, pode ser aproximada por baixo por funções simples. Como $\mathcal{A} \in \mathfrak{S}$, segue $\mathcal{A}=\mathfrak{B}_{\mathrm{c}}(\mathbb{R})$, e $\log \mathrm{S}=\left\{\mathfrak{B}_{\mathrm{c}}(\mathbb{R})\right\}$.

Proposição 6. Seja $\mathfrak{H}$ um espaço de Hilbert e $A \in \mathcal{B}(\mathfrak{H})$ autoadjunto. Para toda $g \in \mathfrak{B}_{\mathrm{c}}(\mathbb{R})$, existe um único operador $g(A) \in \mathcal{B}(\mathfrak{H})$ de forma que

$$
\langle\psi, g(A) \psi\rangle_{\mathfrak{H}}=\int_{\mathbb{R}} g(\lambda) \mathrm{d} \Omega_{\psi}(\lambda) \quad \forall \psi \in \mathfrak{H}
$$

Demonstração. Se para um mesmo $h \in \mathfrak{B}_{\mathrm{c}}(\mathbb{R})$, existem dois operadores $B, B^{\prime} \in \mathcal{B}(\mathfrak{H})$ de forma que

$$
\langle\psi, B \psi\rangle_{\mathfrak{H}}=\int_{\mathbb{R}} h \mathrm{~d} \Omega_{\psi}=\left\langle\psi, B^{\prime} \psi\right\rangle_{\mathfrak{H}} \quad \forall \psi \in \mathfrak{H} \Longrightarrow\left\langle\psi,\left(B-B^{\prime}\right) \psi\right\rangle_{\mathfrak{H}}=0 \quad \forall \psi \in \mathfrak{H} \stackrel{9}{\Longrightarrow} B=B^{\prime}
$$

e a questão da unicidade está resolvida. O conjunto $\mathfrak{X}$ formado pelas funções de $\mathfrak{B}_{\mathrm{c}}(\mathbb{R})$ que cumpre a tese do enunciado contém $\mathrm{C}(\mathbb{R})$ em função do (T.1), e para uma dada sequência de funções $\left(h_{n}\right)_{n \in \mathbb{N}}$ em $\mathfrak{X}$ uniformemente limitada nos compactos e tal que $h_{n} \stackrel{p .}{\longrightarrow} h$, efetua-se

$$
\lim _{n \rightarrow \infty}\left\langle\psi, h_{n}(A) \psi\right\rangle_{\mathfrak{H}}=\lim _{n \rightarrow \infty} \int_{\mathbb{R}} h_{n}(\lambda) \mathrm{d} \Omega_{\psi}(\lambda)=\int_{\mathbb{R}} h(\lambda) \mathrm{d} \Omega_{\psi}(\lambda) \quad \forall \psi \in \mathfrak{H}
$$

pois visto que $\Omega_{\psi}$ é finita e tem suporte no compacto $\sigma(A)$, o teorema da convergência dominada se aplica. Invocando um dos resultados situado no apêndice $\mathrm{A}$, segue $h \in \mathfrak{X}$ e portanto $\mathfrak{X}=\mathfrak{B}_{\mathrm{c}}(\mathbb{R})$.

Ainda nos resta munir $\mathfrak{B}_{\mathrm{c}}(\mathbb{R})$ de uma topologia apropriada, sendo esta a candidata mais promissora:

Definição 7 (Topologia da Convergência Compacta). Se (X, T) é um espaço topológico e $(M, \mathrm{~d})$ um espaço métrico, então

$$
\mathcal{U} \doteq\left\{\mathcal{B}(f, \epsilon ; K): f \in M^{X}, \epsilon>0, K \subseteq X \text { compacto }\right\}
$$

onde

$$
\mathcal{B}(f, \epsilon ; K) \doteq\left\{g \in M^{X}: \sup _{x \in K} \mathrm{~d}(f(x), g(x))<\epsilon\right\}
$$

define uma base de abertos para uma topologia em $M^{X}$, a qual denominamos topologia da convergência compacta.

De fato, $\mathcal{U}$ recobre $M^{X}$ pois toda $u$ neste ultimo está contida em $\mathcal{B}(u, \varrho ; H)$ para qualquer escolha de $\varrho>0$ e $H \subseteq X$ compacto, e se

$$
h \in \mathcal{B}(f, \epsilon ; K) \cap \mathcal{B}(g, \delta ; G)
$$

\footnotetext{
${ }^{9}$ Toda forma sesquilinear $g$ num $\mathbb{C}$-espaço vetorial $\mathcal{X}$ que se anula na diagonal $\{(x, x): x \in \mathcal{X}\}$ se anula por completo por que cumpre a identidade de polarização. Em nosso caso, considere $g(x, y) \doteq\left\langle x,\left(B-B^{\prime}\right) y\right\rangle_{\mathfrak{H}}$.
} 
então

$$
h \in \mathcal{B}(h, \sigma ; K \cup G) \subseteq \mathcal{B}(f, \epsilon ; K) \cap \mathcal{B}(g, \delta ; G)
$$

onde

$$
0<\sigma<\min \left\{\epsilon-\sup _{x \in K} \mathrm{~d}(f(x), h(x)), \delta-\sup _{x \in G} \mathrm{~d}(g(x), h(x))\right\}
$$

A razão pela qual se elege esta terminologia advém do fato de que a convergência nesta topologia equivale a convergência uniforme nos compactos. Com precisão:

Proposição 8. Seja $(X, \mathcal{T})$ um espaço topológico e $(M, \mathrm{~d})$ um espaço métrico. São verdadeiras as seguintes afirmações:

a) O conjunto

$$
\mathcal{U}_{f} \doteq\{\mathcal{B}(f, \epsilon ; K): \epsilon>0, K \subseteq X \text { compacto }\}
$$

define um sistema fundamental de vizinhanças (abertas) para o ponto $f \in M^{X}$ na topologia da convergência compacta.

b) Uma sequência $\left(f_{n}\right)_{n \in \mathbb{N}}$ em $M^{X}$ converge para $f$ na topologia da convergência compacta se, e somente se,

$$
f_{n} \uparrow K \stackrel{u \cdot}{\longrightarrow} f \uparrow K
$$

em todo $K \subseteq X$ compacto.

c) Se $X$ é hemicompacto ${ }^{10}, M^{X}$ satisfaz o primeiro axioma da enumerabilidade.

Demonstração. Para o primeiro item, devemos provar que todo aberto básico de $\mathcal{U}$ contendo uma dada $h \in M^{X}$ abarca um elemento de $\mathcal{U}_{h}$, obrigatoriamente. Pois bem, assuma $h \in \mathcal{B}(g, \epsilon ; K)$ e escolha

$$
0<\delta<\epsilon-\sup _{K} \mathrm{~d}(h(x), g(x))
$$

Para todo $u \in \mathcal{B}(h, \delta ; K)$, tem-se

$$
\mathrm{d}(u(z), g(z)) \leq \mathrm{d}(u(z), h(z))+\mathrm{d}(h(z), g(z))<\delta+\sup _{K} \mathrm{~d}(h(x), g(x)) \quad \forall z \in K
$$

o que significa que

$$
\sup _{K} \mathrm{~d}(u(x), g(x)) \leq \delta+\sup _{K} \mathrm{~d}(h(x), g(x))<\epsilon
$$

Logo $u \in \mathcal{B}(g, \epsilon ; K)$, que por ser arbitrário culmina em

$$
h \in \mathcal{B}(h, \delta ; K) \subseteq \mathcal{B}(g, \epsilon ; K)
$$

Com base no item a), sabe-se que o critério de convergência das $f_{n}$ só precisa ser verificado nos abertos de $\mathcal{U}_{f}$, e daí segue b). Por fim, seja $\mathcal{K}$ uma família enumerável de subconjuntos compactos de $X$ responsável pela hemicompacidade desse espaço. Queremos mostrar que

\footnotetext{
${ }^{10}$ Um espaço topológico $Y$ diz-se hemicompacto quando existe uma família enumerável $\mathcal{K}$ de subconjuntos compactos de $Y$ com a seguinte propriedade: Se $G \subseteq Y$ é compacto $\Longrightarrow G \subseteq K$, para algum $K \in \mathcal{K}$.
} 


$$
\{\mathcal{B}(h, 1 / n, K): n \in \mathbb{N}, K \in \mathcal{K}\}
$$

define um sistema fundamental de vizinhanças (abertas) para um dado ponto $h \in M^{X}$. Efetivamente, escolhido ao acaso $\epsilon>0$ e $G \subseteq X$ compacto, a propriedade arquimediana diz que o conjunto dos números naturais é ilimitado superiormente em $\mathbb{R}$, e por isso existe $k \in \mathbb{N}$ de modo que $1 / k<\epsilon .{ }^{11}$ Tomando $K \in \mathcal{K}$ contendo $G$, temos então

$$
\mathcal{B}(h, 1 / k, K) \subseteq \mathcal{B}(h, \epsilon, G)
$$

pois

$$
\sup _{G} \mathrm{~d}(u(x), h(x)) \leq \sup _{K} \mathrm{~d}(u(x), h(x))<\frac{1}{k}<\epsilon
$$

seja qual for $u \in \mathcal{B}(h, 1 / k, K)$. Uma vez checado que $\mathcal{U}_{h}$ define um sistema fundamental de vizinhanças do ponto $h$, a demonstração está encerrada.

Teorema 9 (Cálculo Funcional Boreliano). Seja $\mathfrak{H}$ um espaço de Hilbert e $A \in \mathcal{B}(\mathfrak{H})$ autoadjunto. Existe um único mapa $\Phi_{A}: \mathfrak{B}_{\mathrm{c}}(\mathbb{R}) \longrightarrow \mathcal{B}(\mathfrak{H})$ cumprindo as seguintes exigências:

a) $\Phi_{A}$ é um homomorfismo de álgebra involutiva unital.

b) $\Phi_{A}$ é contínua na topologia da convergência compacta.

c) $\Phi_{A}(\mathrm{id})=A$.

d) $S e\left(f_{n}\right)_{n \in \mathbb{N}}$ é uma sequência em $\mathfrak{B}_{\mathrm{c}}(\mathbb{R})$ uniformemente limitada nos compactos e tal que $f_{n} \stackrel{p \text {. }}{\longrightarrow}$ $f$, ent $\tilde{a} O \Phi_{A}\left(f_{n}\right) \stackrel{p .}{\longrightarrow} \Phi_{A}(f)$.

Para toda $f \in \mathfrak{B}_{\mathrm{c}}(\mathbb{R})$, também se verifica as seguintes condições suplementares:

e) $\left\|\Phi_{A}(f)\right\|_{\mathrm{op}} \leq \sup _{\sigma(A)}|f|$. [Tal condição fortifica b)]

f) $S e A \psi=\lambda \psi$, ent $\tilde{a} o \Phi_{A}(f) \psi=f(\lambda) \psi$.

g) Se $f \geq 0$, então $\Phi_{A}(f) \geq 0$.

h) Se $B A=A B$ com $B$ limitado, então $\Phi_{A}(f) B=B \Phi_{A}(f)$.

i) Um subespaço fechado $\mathcal{S}$ que é A-invariante também é $\Phi_{A}(f)$-invariante. Em caso afirmativo, vale $\Phi_{A\lceil\mathcal{S}}(f)=\Phi_{A}(f) \uparrow \mathcal{S}$.

Dado um segundo espaço de Hilbert $\mathcal{H}$, acrescenta-se:

j) Se $\pi$ é um homomorfismo de álgebra involutiva unital de $\mathcal{B}(\mathfrak{H})$ em $\mathcal{B}(\mathcal{H})$, então $\Phi_{\pi(A)}=\pi \circ \Phi_{A}$.

k) Se $B \in \mathcal{B}(\mathcal{H})$ é autoadjunto, então $\Phi_{A \oplus B}(f)=\Phi_{A}(f) \oplus \Phi_{B}(f)$.

Demonstração. Seja $\Phi_{A}$ e $\widehat{\Phi}_{A}$ dois mapas de $\mathfrak{B}_{\mathrm{c}}(\mathbb{R})$ em $\mathcal{B}(\mathfrak{H})$ cumprindo as exigências estipuladas no enunciado. De início, já sabemos

$$
\Phi_{A}(\mathrm{id})=\widehat{\Phi}_{A}(\mathrm{id}) \stackrel{\mathrm{a})}{\Longrightarrow} \Phi_{A}(f)=\widehat{\Phi}_{A}(f) \quad \forall f \in \mathfrak{A}^{*}(\mathrm{id})=\mathbb{C}[t \in \mathbb{R}]
$$

\footnotetext{
${ }^{11}$ Do contrário $1 / \epsilon$ seria cota superior de $\mathbb{N}$.
} 
Em sequência, perceba que pelo teorema de Stone-Weierstrass, todo aberto básico contemplado na reunião

$$
\bigcup\left\{\mathcal{U}_{f}: f \in \mathrm{C}(\mathbb{R})\right\}
$$

abarca algum polinômio, e por esse motivo

$$
\overline{\mathbb{C}[t \in \mathbb{R}]}=\mathrm{C}(\mathbb{R})
$$

relativamente à topologia da convergência compacta. Oras, se $\Phi_{A}$ e $\widehat{\Phi}_{A}$ cumprem b) e o contradomínio $\mathcal{B}(\mathfrak{H})$ é Hausdorff,

$$
\mathcal{M} \doteq\left\{f \in \mathfrak{B}_{\mathrm{c}}(\mathbb{R}): \Phi_{A}(f)=\widehat{\Phi}_{A}(f)\right\}
$$

contém $C(\mathbb{R})$. Por fim, se $\left(f_{n}\right)_{n \in \mathbb{N}}$ é uma sequência em $\mathcal{M}$ uniformemente limitada nos compactos e tal que $f_{n} \stackrel{p .}{\longrightarrow} f$, a propriedade d) nos rende

$$
\widehat{\Phi}_{A}(f) \stackrel{p .}{\longleftarrow} \widehat{\Phi}_{A}\left(f_{n}\right)=\Phi_{A}\left(f_{n}\right) \stackrel{p \cdot}{\longrightarrow} \Phi_{A}(f)
$$

e por consequência $\widehat{\Phi}_{A}(f)=\Phi_{A}(f)$, graças a unicidade do limite em espaços Hausdorff. Com isso $f \in \mathcal{M}$, e $\operatorname{logo} \mathcal{M}=\mathfrak{B}_{\mathrm{c}}(\mathbb{R})$. Concluído a unicidade, ponhamos

$$
\begin{aligned}
\Phi_{A}: \mathfrak{B}_{\mathrm{c}}(\mathbb{R}) & \longrightarrow \mathcal{B}(\mathfrak{H}) \\
f & \longmapsto f(A)
\end{aligned}
$$

para resolver a questão da existência. Salvo o produto, a estabilidade das demais estruturas algébricas por $\Phi_{A}$ segue imediatamente da (P.6), a qual nos fornece meios de convertê-las em manipulações com integrais abstratas, que bem sabemos serem compatíveis com as operações ponto-a-ponto. Pois bem, fixada $h \in \mathrm{C}(\mathbb{R})$, sabe-se que

$$
U_{h} \doteq\left\{u \in \mathfrak{B}_{\mathrm{c}}(\mathbb{R}):(u h)(A)=u(A) h(A)\right\}
$$

contém $\mathrm{C}(\mathbb{R})$ pois em virtude do (T.1) aliado a unicidade firmada na (P.6), $u(A)$ se reduz ao cálculo funcional contínuo de $u \uparrow \sigma(A)$ em $A$ quando $u \in \mathrm{C}(\mathbb{R})$, e assim recaímos em terreno onde temos ciência de que tal propriedade é respeitada. Se $\left(u_{n}\right)_{n \in \mathbb{N}}$ é uma sequência em $U_{h}$ uniformemente limitada nos compactos e tal que $u_{n} \stackrel{p .}{\longrightarrow} u$, efetue

$$
\begin{aligned}
&\langle\psi, u(A) h(A) \psi\rangle_{\mathfrak{H}} \stackrel{12}{=} \lim _{n \rightarrow \infty}\left\langle\psi, u_{n}(A) h(A) \psi\right\rangle_{\mathfrak{H}}=\lim _{n \rightarrow \infty}\left\langle\psi,\left(u_{n} h\right)(A) \psi\right\rangle_{\mathfrak{H}}= \\
& \quad=\lim _{n \rightarrow \infty} \int_{\mathbb{R}} u_{n} h \mathrm{~d} \Omega_{\psi}=\int_{\mathbb{R}} u h \mathrm{~d} \Omega_{\psi}=\langle\psi,(u h)(A) \psi\rangle_{\mathfrak{H}}
\end{aligned}
$$

pela justifica de sempre: Como $\Omega_{\psi}$ é finita e tem suporte no compacto $\sigma(A)$, o teorema da convergência dominada está liberado. Sendo $\psi \in \mathfrak{H}$ arbitrário, provamos

$$
(u h)(A)=u(A) h(A)
$$

$\operatorname{logo} u \in U_{h}$, e portanto $U_{h}=\mathfrak{B}_{\mathrm{c}}(\mathbb{R})$. Pra concluir, $h$ também é qualquer, e com isso

$$
V_{g} \doteq\left\{u \in \mathfrak{B}_{\mathrm{c}}(\mathbb{R}): u(A) g(A)=(u g)(A)\right\} \supseteq \mathrm{C}(\mathbb{R})
$$

\footnotetext{
${ }^{12}$ Aplique polarização na forma sesquilinear $g(x, y) \doteq\left\langle x, u_{n}(A) y\right\rangle_{\mathfrak{H}}$, e com isso obtenha expressões que podem ser convertidas em integrais com respeito a alguma medida espectral. Feito isso, aplique o teorema da convergência dominada em cada uma das partes, e ao final reagrupe os termos remanescente novamente com polarização.
} 
seja qual for $g \in \mathfrak{B}_{\mathrm{c}}(\mathbb{R})$. A condição faltante para que o lema da classe monótona funcional novamente se aplique segue trocando $h$ por $g$ no caso anterior. Constatado que $\Phi_{A}$ é um homomorfismo, na sequência temos

$$
\begin{aligned}
&\|h(A) \psi\|_{\mathfrak{H}}^{2}=\left\langle\psi, h(A)^{*}\right.h(A) \psi\rangle_{\mathfrak{H}}=\langle\psi, \bar{h}(A) h(A) \psi\rangle_{\mathfrak{H}}=\langle\psi,(\bar{h} h)(A) \psi\rangle_{\mathfrak{H}}= \\
& \quad=\left\langle\psi,|h|^{2}(A) \psi\right\rangle_{\mathfrak{H}}=\int_{\mathbb{R}}|h|^{2} \mathrm{~d} \Omega_{\psi} \leq \Omega_{\psi}(\mathbb{R}) \sup _{\sigma(A)}|h|^{2}=\left(\sup _{\sigma(A)}|h|\right)^{2}\|\psi\|_{\mathfrak{H}}^{2} \quad \forall \psi \in \mathfrak{H}
\end{aligned}
$$

qualquer que seja $h \in \mathfrak{B}_{\mathrm{c}}(\mathbb{R})$, provando e). Dado que a reta real é hemicompacta ${ }^{13}$ e o primeiro axioma da enumerabilidade integra uma propriedade topológica hereditária, $\mathfrak{B}_{\mathrm{c}}(\mathbb{R})$ é primeiro contável, e por isso vale a caracterização da continuidade por sequências. Por certo, se $\left(f_{n}\right)_{n \in \mathbb{N}}$ é uma sequência em $\mathfrak{B}_{\mathrm{c}}(\mathbb{R})$ convergindo à $f$ na topologia da convergência compacta, em particular

$$
f_{n}\left\lceil\sigma(A) \stackrel{u .}{\longrightarrow} f \uparrow \sigma(A) \Longrightarrow\left\|f_{n}(A)-f(A)\right\|_{\mathrm{op}}=\left\|\left(f_{n}-f\right)(A)\right\|_{\mathrm{op}} \leq \sup _{\sigma(A)}\left|f_{n}-f\right| \longrightarrow 0\right.
$$

e com isso provou-se b). Supondo agora que as $f_{n}$ nos confere uma sequência de funções em $\mathfrak{B}_{\mathrm{c}}(\mathbb{R})$ uniformemente limitada nos compactos e tal que $f_{n} \stackrel{p .}{\longrightarrow} f$, efetuamos

$$
\left\|f_{n}(A) \psi-f(A) \psi\right\|_{\mathfrak{H}}^{2}=\left\|\left(f_{n}-f\right)(A) \psi\right\|_{\mathfrak{H}}^{2}=\left\langle\psi,\left|f_{n}-f\right|^{2}(A) \psi\right\rangle_{\mathfrak{H}}=\int_{\mathbb{R}}\left|f_{n}-f\right|^{2} \mathrm{~d} \Omega_{\psi} \longrightarrow 0 \quad \forall \psi \in \mathfrak{H}
$$

o que quer dizer $f_{n}(A) \stackrel{p .}{\longrightarrow} f(A)$, donde d). Por causa do (C.4),

$$
f \in \mathfrak{B}_{\mathrm{c}}(\mathbb{R}): f \geq 0 \Longrightarrow\langle\psi, f(A) \psi\rangle_{\mathfrak{H}}=\int_{\mathbb{R}} f \mathrm{~d} \Omega_{\psi} \geq 0 \quad \forall \psi \in \mathfrak{H} \Longrightarrow f(A) \geq 0
$$

e assim segue g). Adiante, $A \psi=\lambda \psi$ ocasiona

$$
0=\langle\psi,(A-\lambda \mathbb{1}) \psi\rangle_{\mathfrak{H}}=\int_{\mathbb{R}}(t-\lambda) \mathrm{d} \Omega_{\psi}(t)
$$

e que só é possível quando $t=\lambda$ em $\Omega_{\psi}$-quase toda parte. Mas então $f(t)=f(\lambda)$ em $\Omega_{\psi}$-quase toda parte, e daí

$$
\|f(A) \psi-f(\lambda) \psi\|_{\mathfrak{H}}^{2}=\left\langle\psi,|f-f(\lambda)|^{2}(A) \psi\right\rangle_{\mathfrak{H}}=\int_{\mathbb{R}}|f(t)-f(\lambda)|^{2} \mathrm{~d} \Omega_{\psi}(t)=0
$$

Logo $f(A) \psi=f(\lambda) \psi$, e o item $\mathrm{f}$ ) também está provado. A seguir, veja que

$$
\mathcal{N} \doteq\left\{f \in \mathfrak{B}_{\mathrm{c}}(\mathbb{R}): B f(A)=f(A) B\right\}
$$

contém $C(\mathbb{R})$ por que tal propriedade se verifica para o cálculo funcional contínuo, e se $\left(f_{n}\right)_{n \in \mathbb{N}}$ configura uma sequência em $\mathcal{N}$ uniformemente limitada nos compactos e tal que $f_{n} \stackrel{p \text {. }}{\longrightarrow} f$, em particular $f_{n}(A) B \stackrel{p .}{\longrightarrow} f(A) B$, mas também $B f_{n}(A) \stackrel{p .}{\longrightarrow} B f(A)$ por que $B$ é limitado. Em atenção a unicidade do limite em espaços métricos, ficamos com $f \in \mathcal{N}$, e finalmente h).

O conjunto $\mathcal{M} \doteq\left\{f \in \mathfrak{B}_{\mathrm{c}}(\mathbb{R}): \mathcal{S}\right.$ é $f(A)$-invariante $\}$ contém as contínuas pela mesma razão de agora pouco, e se $\left(f_{n}\right)_{n \in \mathbb{N}}$ é uma sequência em $\mathcal{M}$ uniformemente limitada nos compactos e tal que $f_{n} \stackrel{p .}{\longrightarrow} f$, atribui-se

$$
f_{n}(A) \psi \in \mathcal{S} \quad \forall \psi \in \mathcal{S}, n \in \mathbb{N} \Longrightarrow f(A) \psi=\lim _{n} f_{n}(A) \psi \in \overline{\mathcal{S}}=\mathcal{S} \quad \forall \psi \in \mathcal{S}
$$

\footnotetext{
${ }^{13}$ Basta considerar a família de intervalos $[-n, n]$, com $n \in \mathbb{N}$.
} 
$\operatorname{logo} f \in \mathcal{M}$, e enfim $\mathcal{M}=\mathfrak{B}_{\mathrm{c}}(\mathbb{R})$. A segunda parte do enunciado segue do item subsequente com

$$
\begin{aligned}
\pi: \mathcal{B}(\mathfrak{H}) & \longrightarrow \mathcal{B}(\mathcal{S}) \\
B & \longmapsto B \uparrow S
\end{aligned}
$$

e pra terminar, tanto $\pi \circ \Phi_{A}$ quanto $\Phi_{A} \oplus \Phi_{B}$ verificam quase que trivialmente os quatro primeiros itens que definem univocamente o cálculo funcional boreliano de $\pi(A)$ e $A \oplus B$, respectivamente. ${ }^{14}$

Com isso ampliamos nosso repertório de funções para o qual podemos produzir algum tipo de cálculo funcional em um operador autoadjunto limitado.

Definição 10 (Projetor Espectral). Seja $\mathfrak{H}$ um espaço de Hilbert e $A \in \mathcal{B}(\mathfrak{H})$ autoadjunto. Dizemos que

$$
\mathrm{P}_{G}(A) \doteq \chi_{G}(A)
$$

é o projetor espectral de $A$ no boreliano $G$ de $\mathbb{R}$.

Aplicando o cálculo funcional na expressão $\chi_{G}^{2}=\chi_{G}=\overline{\chi_{G}}$, obtém-se $\mathrm{P}_{G}(A)^{2}=\mathrm{P}_{G}(A)=\mathrm{P}_{G}(A)^{*}$, o que o caracteriza como um projetor ortogonal em $\mathfrak{H}$.

Proposição 11. Seja $\mathfrak{H}$ um espaço de Hilbert, $A \in \mathcal{B}(\mathfrak{H})$ autoadjunto e $E, G$ borelianos de $\mathbb{R}$. São verdadeiras as seguintes afirmações:

a) $\mathrm{P}_{\varnothing}(A)=0, \mathrm{P}_{\mathbb{R}}(A)=\mathbb{1}, \mathrm{P}_{G}(A) \mathrm{P}_{E}(A)=\mathrm{P}_{G \cap E}(A)$

b) $S e E \subseteq G$, então $\mathrm{P}_{E}(A) \leq \mathrm{P}_{G}(A)$

c) Se $E \cap G=\varnothing$, então $\mathrm{P}_{G \cup E}(A)=\mathrm{P}_{E}(A)+\mathrm{P}_{G}(A)$

d) $S e G \cap \sigma(A)=\varnothing$, então $\mathrm{P}_{G}(A)=0$

e) Para toda coleção $\left\{G_{n}\right\}_{n \in \mathbb{N}}$ de borelianos de $\mathbb{R}$ dois-a-dois disjuntos, tem-se

$$
\sum_{1}^{n} \mathrm{P}_{G_{i}}(A) \stackrel{p .}{\longrightarrow} \mathrm{P}_{\bigcup_{1}^{\infty} G_{n}}(A)
$$

f) $\lambda$ é autovalor de $A$ se, e somente se, $\mathrm{P}_{\{\lambda\}}(A) \neq 0$. Em caso afirmativo, $\mathrm{P}_{\{\lambda\}}(A)$ é precisamente o projetor ortogonal de $\mathfrak{H}$ em $\mathfrak{H}_{\lambda}(A)$.

Demonstração. Os três primeiros itens podem ser obtidos passando o cálculo nas expressões

$$
\chi_{\varnothing} \equiv 0 \quad, \quad \chi_{\mathbb{R}} \equiv 1 \quad, \quad \chi_{G \cap E}=\chi_{G} \chi_{E} \quad, \quad \chi_{G \backslash E}=\chi_{G}-\chi_{E} \quad, \quad \chi_{G \cup E}=\chi_{G}+\chi_{E}
$$

sendo que na penúltima supomos $E \subseteq G$, e na última $G \cap E=\varnothing$. Na sequência,

$$
\operatorname{supp}\left(\Omega_{\psi}\right)=\sigma(A) \quad \forall \psi \in \mathfrak{H} \Longrightarrow\left\langle\psi, \mathrm{P}_{G}(A) \psi\right\rangle_{\mathfrak{H}}=\int_{\mathbb{R}} \chi_{G} \mathrm{~d} \Omega_{\psi}=\Omega_{\psi}(G)=0 \quad \forall \psi \in \mathfrak{H} \Longrightarrow \mathrm{P}_{G}(A)=0
$$

\footnotetext{
${ }^{14}$ Vale lembrar que a continuidade de $\pi$ é automática, uma vez que seu domínio e contradominío são álgebras $\mathrm{C}^{*}$.
} 
e com isso provamos o item d). Dado que $\left(\sum_{1}^{n} \chi_{G_{i}}\right)_{n \in \mathbb{N}}$ é uma sequência em $\mathfrak{B}_{\mathrm{c}}(\mathbb{R})$ uniformemente limitada (por 1) e tal que

$$
\sum_{1}^{n} \chi_{G_{i}} \stackrel{p .}{\longrightarrow} \chi_{\bigcup_{1}^{\infty} G_{i}}
$$

segue

$$
\sum_{1}^{n} \mathrm{P}_{G_{i}}(A)=\Phi_{A}\left(\sum_{1}^{n} \chi_{G_{i}}\right) \stackrel{p .}{\longrightarrow} \Phi_{A}\left(\chi_{\cup_{1}^{\infty} G_{n}}\right)=\mathrm{P}_{\cup_{1}^{\infty} G_{n}}(A)
$$

e portanto e). Para o ultimo item, é suficiente que se estabeleça $\operatorname{Im}\left(\mathrm{P}_{\{\lambda\}}(A)\right)=\mathfrak{H}_{\lambda}(A)$. Com efeito, passando o cálculo na expressão $t \chi_{\{\lambda\}}(t)=\lambda \chi_{\{\lambda\}}(t)$, válida para todo $t$ real, obtém-se

$$
A \mathrm{P}_{\{\lambda\}}(A)=\lambda \mathrm{P}_{\{\lambda\}}(A)
$$

e por consequência

$$
\psi \in \operatorname{Im}\left(\mathrm{P}_{\{\lambda\}}(A)\right) \Longrightarrow \psi=\mathrm{P}_{\{\lambda\}}(A) \psi \Longrightarrow A \psi=\lambda \psi \Longrightarrow \psi \in \mathfrak{H}_{\lambda}(A)
$$

Reciprocamente,

$$
\psi \in \mathfrak{H}_{\lambda}(A) \Longrightarrow A \psi=\lambda \psi \Longrightarrow \mathrm{P}_{\{\lambda\}}(A) \psi=\chi_{\{\lambda\}}(\lambda) \psi=\psi \Longrightarrow \psi \in \operatorname{Im}\left(\mathrm{P}_{\{\lambda\}}(A)\right)
$$

por causa do item $\mathrm{f})$ do (T.9).

Corolário 12. Seja $\mathfrak{H}$ um espaço de Hilbert e $A \in \mathcal{B}(\mathfrak{H})$ autoadjunto. Todo ponto isolado de $\sigma(A)$ é necessariamente um autovalor de $A$.

Demonstração. Seja, pois, $\lambda \in \mathbb{R}$ e $\varepsilon>0$ de forma que

$$
\sigma(A) \cap(\lambda-\varepsilon, \lambda+\varepsilon)=\{\lambda\}
$$

Caso $\lambda$ não seja um autovalor de $A$, a proposição anterior garante

$$
\mathrm{P}_{(\lambda-\varepsilon, \lambda+\varepsilon)}(A)=\mathrm{P}_{\{\lambda\}}(A)=0
$$

e por isso que $f_{\varepsilon} \in \mathfrak{B}_{\mathrm{c}}(\mathbb{R})$ definida por

$$
f_{\varepsilon}(\lambda)=\left\{\begin{array}{cl}
(t-\lambda)^{-1} & , t \in \mathbb{R} \backslash(\lambda-\varepsilon, \lambda+\varepsilon) \\
0 & , t \in(\lambda-\varepsilon, \lambda+\varepsilon)
\end{array}\right.
$$

ao cumprir

$$
(t-\lambda) f_{\varepsilon}(\lambda)=\chi_{\mathbb{R} \backslash(\lambda-\varepsilon, \lambda+\varepsilon)}(t)=1-\chi_{(\lambda-\varepsilon, \lambda+\varepsilon)}(t) \quad \forall t \in \mathbb{R}
$$

ocasiona

$$
(A-\lambda \mathbb{1}) f_{\varepsilon}(A)=\mathbb{1}-\mathrm{P}_{(\lambda-\varepsilon, \lambda+\varepsilon)}(A)=\mathbb{1}-0=\mathbb{1}
$$


Mas dado que $A-\lambda \mathbb{1}$ e $f_{\varepsilon_{0}}(A)$ comutam $^{15}$, concluímos $\lambda \notin \sigma(A)$, e portanto uma contradição.

Teorema 13. Seja $\mathfrak{H}$ um espaço de Hilbert e $A \in \mathcal{B}(\mathfrak{H})$ autoadjunto. São equivalentes:

a) $\lambda$ é um ponto no espectro de $A$.

b) Para todo $\varepsilon>0, \mathrm{P}_{(\lambda-\varepsilon, \lambda+\varepsilon)}(A) \neq 0$.

c) Existe uma sequência $\left(\psi_{n}\right)_{n \in \mathbb{N}}$ em $\mathbb{S}^{1}(\mathfrak{H})$ de forma que $\lim _{n}\left\|(A-\lambda \mathbb{1}) \psi_{n}\right\|_{\mathfrak{H}}=0$.

Demonstração. A implicação a) $\Longrightarrow$ b) já foi provada no corolário acima por contra-positiva. Provemos que b) $\Longrightarrow$ c). De fato, tomando $f_{n} \in \mathfrak{B}_{\mathrm{c}}(\mathbb{R})$ de modo que

$$
f_{n}(t)=(t-\lambda)^{2} \chi_{\left(\lambda-\frac{1}{n}, \lambda+\frac{1}{n}\right)}(t), \quad t \in \mathbb{R}
$$

e $\psi_{n}$ um vetor unitário na imagem de $\mathrm{P}_{\left(\lambda-\frac{1}{n}, \lambda+\frac{1}{n}\right)}(A)$, temos

$$
\begin{aligned}
\left\|(A-\lambda \mathbb{1}) \psi_{n}\right\|_{\mathfrak{H}}^{2}=\left\|(A-\lambda \mathbb{1}) \mathrm{P}_{\left(\lambda-\frac{1}{n}, \lambda+\frac{1}{n}\right)}(A) \psi_{n}\right\|_{\mathfrak{H}}^{2}=\left\langle\psi_{n},(A-\lambda \mathbb{1})^{2} \mathrm{P}_{\left(\lambda-\frac{1}{n}, \lambda+\frac{1}{n}\right)}(A)^{2} \psi_{n}\right\rangle_{\mathfrak{H}} \leq \\
\leq\left\|(A-\lambda \mathbb{1})^{2} \mathrm{P}_{\left(\lambda-\frac{1}{n}, \lambda+\frac{1}{n}\right)}(A)\right\|\left\|_{\text {op }}|| \psi_{n}\right\|_{\mathfrak{H}}^{2} \leq \sup _{\mathbb{R}}\left|f_{n}\right| \leq \frac{1}{n^{2}} \longrightarrow 0
\end{aligned}
$$

Para mostrar c) $\Longrightarrow$ a), basta observar que se $\lambda$ não fosse um ponto espectral, teríamos

$$
1=\left\|\psi_{n}\right\|_{\mathfrak{H}}=\left\|(A-\lambda \mathbb{1})^{-1}(A-\lambda \mathbb{1}) \psi_{n}\right\|_{\mathfrak{H}} \leq\left\|(A-\lambda \mathbb{1})^{-1}\right\|_{\text {op }}\left\|(A-\lambda \mathbb{1}) \psi_{n}\right\|_{\mathfrak{H}} \longrightarrow 0
$$

o que é um absurdo.

Definição 14 (Espaço Espectral Absolutamente Contínuo). Seja $\mathfrak{H}$ um espaço de Hilbert. O espaço espectral absolutamente contínuo de um operador autoajunto $A \in \mathcal{B}(\mathfrak{H})$ é definido como

$$
\mathfrak{H}_{a c}(A) \doteq\left\{\psi \in \mathfrak{H}: \Omega_{\psi} \ll \mathfrak{m}\right\}
$$

Este conceito terá relevância notória apenas na seção 3.2, com o advento dos operadores de onda. Contudo, um exemplo proeminente é discutido no (Ex.42) à frente.

Proposição 15. Seja $A$ e $B$ operadores limitados e autoadjuntos agindo nos espaços de Hilbert $\mathfrak{H} e$ $\mathcal{H}$, respectivamente. São verdadeiras as seguintes sentenças:

a) $\mathfrak{H}_{a c}(A)$ configura um subespaço fechado e A-invariante de $\mathfrak{H}$.

b) Se $B=U^{*} A U \operatorname{com} U \in \mathcal{B}(\mathcal{H}, \mathfrak{H})$ unitário, então $\mathfrak{H}_{\mathrm{ac}}(A) \simeq_{U} \mathcal{H}_{\mathrm{ac}}(B)$.

c) Se $\mathcal{S}$ é um subespaço fechado e $A$-invariante de $\mathfrak{H}$, então $\mathfrak{H}_{\mathrm{ac}}(A) \cap \mathcal{S}=\mathcal{S}_{\mathrm{ac}}(A\lceil\mathcal{S})$.

d) $(\mathfrak{H} \oplus \mathcal{H})_{\text {ac }}(A \oplus B)=\mathfrak{H}_{\mathrm{ac}}(A) \oplus \mathcal{H}_{\mathrm{ac}}(B)$.

e) Se $P_{\mathrm{ac}}(A)$ denota o projetor ortogonal de $\mathfrak{H}$ em $\mathfrak{H}_{\mathrm{ac}}(A)$, então $A P_{\mathrm{ac}}(A)=P_{\mathrm{ac}}(A) A$.

\footnotetext{
${ }^{15} \operatorname{Im}\left(\Phi_{A}\right)$ configura uma subálgebra comutativa de $\mathcal{B}(\mathfrak{H})$ por que $\mathfrak{B}_{\mathrm{c}}(\mathbb{R})$ é comutativa e $\Phi_{A}$ um homomorfismo.
} 
Demonstração. Se $\psi$ é limite de uma sequência de vetores $\left(\psi_{n}\right)_{n \in \mathbb{N}}$ em $\mathfrak{H}_{\mathrm{ac}}(A)$ e $G$ um boreliano de $\mathbb{R}$ com medida de Lebesgue nula, tem-se

$$
\lim _{n \rightarrow \infty} \Omega_{\psi_{n}}(G)=\lim _{n \rightarrow \infty} \int_{\mathbb{R}} \chi_{G} \mathrm{~d} \Omega_{\psi_{n}}=\lim _{n \rightarrow \infty}\left\langle\psi_{n}, \mathrm{P}_{G}(A) \psi_{n}\right\rangle_{\mathfrak{H}}=\left\langle\psi, \mathrm{P}_{G}(A) \psi\right\rangle_{\mathfrak{H}}=\int_{\mathbb{R}} \chi_{G} \mathrm{~d} \Omega_{\psi}=\Omega_{\psi}(G)
$$

por conta da continuidade das aplicações envolvidas, mas também

$$
\Omega_{\psi_{n}} \ll \mathfrak{m} \Longrightarrow \Omega_{\psi_{n}}(G)=0 \Longrightarrow \lim _{n \rightarrow \infty} \Omega_{\psi_{n}}(G)=0
$$

Em atenção a unicidade do limite de sequências numéricas, $G$ tem medida espectral (subordinada ao vetor $\psi$ ) nula, e por ter sido escolhido ao acaso obtemos

$$
\Omega_{\psi} \ll \mathfrak{m} \Longrightarrow \psi \in \mathfrak{H}_{\mathrm{ac}}(A)
$$

provando que $\mathfrak{H}_{\mathrm{ac}}(A)$ é fechado em $\mathfrak{H}$. Para todo $\psi \in \mathfrak{H}, \varphi \in \mathcal{H}, \phi \in \mathcal{S}$ e $G \in \mathfrak{B}_{\mathbb{R}}$,

$$
\Omega_{A \psi}(G)=\int_{\mathbb{R}} \chi_{G} \mathrm{~d} \Omega_{A \psi}=\left\langle A \psi, \mathrm{P}_{G}(A) A \psi\right\rangle_{\mathfrak{H}}=\left\langle\psi, A^{2} \mathrm{P}_{G}(A) \psi\right\rangle_{\mathfrak{H}}=\int_{G} t^{2} \mathrm{~d} \Omega_{\psi}(t) \leq \Omega_{\psi}(G) \sup _{G} t^{2}
$$

conclui a prova do item a),

$$
\begin{aligned}
\Omega_{U^{*} \psi, B}(G)=\int_{\mathbb{R}} \chi_{G} \mathrm{~d} \Omega_{U^{*} \psi, B}=\left\langle U^{*} \psi, \mathrm{P}_{G}(B) U^{*} \psi\right\rangle_{\mathcal{H}} \stackrel{16}{=}\left\langle U^{*} \psi, U^{*} \mathrm{P}_{G}(A) U U^{*} \psi\right\rangle_{\mathcal{H}} & = \\
& =\left\langle\psi, \mathrm{P}_{G}(A) \psi\right\rangle_{\mathfrak{H}}=\int_{\mathbb{R}} \chi_{G} \mathrm{~d} \Omega_{\psi, A}=\Omega_{\psi, A}(G)
\end{aligned}
$$

demonstra b),

$$
\Omega_{\phi, A \uparrow \mathcal{S}}(G)=\int_{\mathbb{R}} \chi_{G} \mathrm{~d} \Omega_{\phi, A \uparrow \mathcal{S}}=\left\langle\phi, \mathrm{P}_{G}(A\lceil\mathcal{S}) \phi\rangle_{\mathfrak{H}}=\left\langle\phi, \mathrm{P}_{G}(A) \phi\right\rangle_{\mathfrak{H}}=\int_{\mathbb{R}} \chi_{G} \mathrm{~d} \Omega_{\phi, A}=\Omega_{\phi, A}(G)\right.
$$

comprova c), e pra não tornar o argumento muito repetitivo, d) fica para o leitor. Por fim, e) é consequência deveras de que $\mathfrak{H}_{\text {ac }}(A)$ é $A$-invariante.

\subsection{Critério de Weyl}

"Concentre todos seus pensamentos na tarefa que está realizando. Os raios de sol não queimam até que sejam colocados em foco."

Graham Bell ${ }^{17}$

Definição 16 (Convergência Fraca). Dizemos que uma sequência de vetores $\left(x_{n}\right)_{n \in \mathbb{N}}$ em um $\mathbb{K}$-espaço vetorial normado $\mathcal{X}$ converge fracamente para o vetor $x \in \mathcal{X}$ quando

$$
\lim _{n \rightarrow \infty} \ell\left(x_{n}\right)=\ell(x) \quad \forall \ell \in X^{*}
$$

Em caso afirmativo, escreve-se $x_{n} \stackrel{\text { w. }}{\longrightarrow} x$.

${ }^{16}$ Basta aplica o $(\mathrm{T} .9) \operatorname{com} \mathcal{B}(\mathfrak{H}) \ni T \stackrel{{ }^{\pi}}{\longmapsto} U^{*} T U \in \mathcal{B}(\mathcal{H})$.

${ }^{17}$ Alexander Graham Bell, $\odot(03 / 03 / 1847)-\dagger(02 / 08 / 1922)$ 
Em contraste com a definição que acabamos de enunciar, é comum neste contexto denominar por convergência forte aquela dada de maneira ordinária pela norma de $X$. Reunimos os resultados básicos acerca desta última definição na seguinte proposição:

Proposição 17. Se $\mathcal{X}$ e qualificam dois $\mathbb{K}$-espaços vetoriais normados, então:

a) Toda sequência fortemente convergente é fracamente convergente. Neste caso, o limite forte e fraco coincidem.

b) Toda subsequência de uma sequência fracamente convergente converge fracamente, e para o mesmo limite fraco da sequência ordinária.

c) O limite fraco, quando existe, é único.

d) Operadores limitados de $\mathcal{X}$ em $\boldsymbol{Y}$ transformam sequências fracamente convergente em sequências fracamente convergente.

e) Toda sequência fracamente convergente é limitada.

f) Operadores compactos de $x$ em $y$ transformam sequências fracamente convergente em sequências fortemente convergente.

Se $\chi$ for um espaço de Hilbert, acrescenta-se

g) Toda sequência ortonormal converge fracamente para o vetor nulo.

Demonstração. Nos encarregamos apenas das asserções que julgamos ser não triviais. Com efeito, se $\left(u_{n}\right)_{n \in \mathbb{N}}$ é uma sequência em $\mathcal{X}$ que tem $u$ e $u^{\prime}$ como limite fraco, cada $\left(\ell\left(u_{n}\right)\right)_{n \in \mathbb{N}}$ tem $\ell(u)$ e $\ell\left(u^{\prime}\right)$ como limite forte, e por unicidade

$$
\begin{aligned}
\ell(u)=\ell\left(u^{\prime}\right) \quad \forall \ell \in X^{*} \Longrightarrow \varkappa\left(u-u^{\prime}\right) \ell=\ell\left(u-u^{\prime}\right)=0 \quad \forall \ell \in X^{*} \Longrightarrow \\
\Longrightarrow \varkappa\left(u-u^{\prime}\right)=0 \Longrightarrow\left\|u-u^{\prime}\right\|_{x}=\left\|\varkappa\left(u-u^{\prime}\right)\right\|_{\text {op }}=0 \Longrightarrow u=u^{\prime}
\end{aligned}
$$

Por força da convergência fraca, também sabemos que para cada $\ell \in X^{*}$, a sequência numérica $\left(\ell\left(u_{n}\right)\right)_{n \in \mathbb{N}}$ é limitada, e portanto existe $c_{\ell}>0$ de forma que

$$
\left|\varkappa\left(u_{n}\right) \ell\right|=\left|\ell\left(u_{n}\right)\right|<c_{\ell} \quad \forall n \in \mathbb{N}
$$

Dado que $\left\{\varkappa\left(x_{n}\right)\right\}_{n \in \mathbb{N}}$ se mostrou uma família pontualmente limitada de funcionais definidos no espaço de Banach $X^{*}$, o saudoso teorema de Banach-Steinhauss nos fornece $c>0$ tal que

$$
\left\|u_{n}\right\|_{x}=\left\|\varkappa\left(u_{n}\right)\right\|_{\text {op }}<c \quad \forall n \in \mathbb{N}
$$

Agora, suponha que para um dado $K \in \mathrm{K}(\mathcal{X}, y),\left(K\left(u_{n}\right)\right)_{n \in \mathbb{N}}$ não convirja fortemente pra $K(u)$. Logo encontramos uma subsequência $\left(K\left(u_{n_{k}}\right)\right)_{k \in \mathbb{N}}$ e $r>0$ de modo que

$$
K\left(u_{n_{k}}\right) \notin B_{r}(K(u)) \quad \forall k \in \mathbb{N}
$$

Por conta da limitação de $\left(u_{n_{k}}\right)_{k \in \mathbb{N}}$ e compacidade de $K,\left(K\left(u_{n_{k}}\right)\right)_{k \in \mathbb{N}}$ possui uma subsequência $\left(K\left(u_{n_{k_{j}}}\right)\right)_{j \in \mathbb{N}}$ convergindo fortemente (e portanto fracamente) para algum $y \in \mathcal{y}$, diferente de $K(u)$ graças a expressão acima. Todavia 


$$
u_{n} \stackrel{\text { w. }}{\longrightarrow} u \stackrel{\text { d) }}{\Longrightarrow} K\left(u_{n}\right) \stackrel{\text { w. }}{\longrightarrow} K(u) \stackrel{\text { b) }}{\Longrightarrow} K\left(u_{n_{k_{j}}}\right) \stackrel{\text { w. }}{\longrightarrow} K(u) \stackrel{\text { c) }}{\Longrightarrow} y=K(u)
$$

e caímos em uma contradição. Por ultimo, $\left(\left\langle x, u_{n}\right\rangle_{\mathfrak{H}}\right)_{n \in \mathbb{N}}$ converge à 0 quando os $u_{n}$ são ortonormais pois, em atenção a desigualdade de Bessel, é termo geral de uma série convergente.

É curioso como muito dos resultados partilhados entre a convergência forte e fraca se apresentam como consequência óbvia para uma, e um fato altamente não trivial a outra.

No que segue, apresentamos uma versão simplificada do teorema de Banach-Alaoglu que pode e deve ser encarada como uma generalização (no sentido fraco) do teorema de Bolzanno-Weierstrass.

Teorema 18 (Banach-Alaoglu). Toda sequência limitada em um espaço de Hilbert separável $\mathfrak{H}$ admite uma subsequência fracamente convergente.

Demonstração. Seja então $\left(x_{n}\right)_{n \in \mathbb{N}}$ uma sequência limitada. Como $\mathfrak{H}$ é separável e

$$
\mathfrak{H} \ni v \longmapsto v^{*} \in \mathfrak{H}^{*}
$$

contínua sobrejetora, segue que $\mathfrak{H}^{*}$ é separável, e por isso admite um subconjunto denso e enumerável Q. Para auxiliar o argumento subsequente, consideremos $f$ uma enumeração de Q. Pois bem, dado que transformações lineares limitadas de modo geral enviam conjuntos limitados em limitados, $\left(f_{1}\left(x_{n}\right)\right)_{n \in \mathbb{N}}$ nos confere uma sequência numérica limitada, e por força do teorema de Bolzanno-Weierstrass admite uma subsequência convergente $\left(f_{1}\left(x_{1, n}\right)\right)_{n \in \mathbb{N}}$. Só que por ser subsequência de $\left(x_{n}\right)_{n \in \mathbb{N}},\left(x_{1, n}\right)_{n \in \mathbb{N}}$ também é limitada, e com maior razão $\left(f_{2}\left(x_{1, n}\right)\right)_{n \in \mathbb{N}}$, possuindo assim uma subsequência convergente $\left(f_{2}\left(x_{2, n}\right)\right)_{n \in \mathbb{N}}$. Prosseguindo de forma indutiva, obtemos uma coleção de sequências $\left(x_{k, n}\right)_{n \in \mathbb{N}}$ verificando as seguintes alegações:

- $\left(x_{k+1, n}\right)_{n \in \mathbb{N}}$ é subsequência de $\left(x_{k, n}\right)_{n \in \mathbb{N}}$ para todo $k \in \mathbb{N}$.

- $\left(f_{k}\left(x_{k, n}\right)\right)_{n \in \mathbb{N}}$ é convergente para todo $k \in \mathbb{N}$

Pelo argumento padrão da sequência diagonal, $\left(z_{k}=x_{k, k}\right)_{k \in \mathbb{N}}$ define uma subsequência comum a todas $\left(x_{k, n}\right)_{n \in \mathbb{N}}$, e portanto aquela para o qual $\left(\varphi\left(z_{k}\right)\right)_{k \in \mathbb{N}}$ converge sempre que $\varphi \in \mathcal{Q}$. Concluído nosso primeiro objetivo, o próximo baseia-se em tirar proveito da densidade de 2 com o intuito de propagar essa propriedade à todo funcional compreendido no dual de $\mathfrak{H}$.

Com efeito, se $\varphi \in \mathfrak{H}^{*}$ pode ser aproximado por uma sequência de funcionais $\left(\varphi_{n}\right)_{n \in \mathbb{N}}$ em $\mathcal{Q}$, então para um dado $\varepsilon>0$, tem que existir $n_{0} \in \mathbb{N}$ de forma que

$$
\left\|\varphi-\varphi_{n}\right\|_{\text {op }}<\frac{\varepsilon}{4 M}
$$

sempre que $n>n_{0}$. [A constante $M>0$ é um majorante da norma dos $\left.x_{n}\right]$ Sendo $\left(\varphi_{n_{0}+1}\left(z_{k}\right)\right)_{k \in \mathbb{N}}$ de Cauchy, também se obtém $k_{0} \in \mathbb{N}$ para o qual

$$
k, l>k_{0} \Longrightarrow\left|\varphi_{n_{0}+1}\left(x_{k}\right)-\varphi_{n_{0}+1}\left(x_{l}\right)\right|<\frac{\varepsilon}{2}
$$


e portanto

$$
\begin{aligned}
\left|\varphi\left(z_{k}\right)-\varphi\left(z_{l}\right)\right| & \leq\left|\varphi\left(z_{k}\right)-\varphi_{n_{0}+1}\left(z_{k}\right)\right|+\left|\varphi_{n_{0}+1}\left(z_{k}\right)-\varphi_{n_{0}+1}\left(z_{l}\right)\right|+\left|\varphi_{n_{0}+1}\left(z_{l}\right)-\varphi\left(z_{l}\right)\right| \\
& <\left\|\varphi-\varphi_{n_{0}+1}||_{\text {op }}|| z_{k}||_{\mathfrak{H}}+\left|\varphi_{n_{0}+1}\left(z_{k}\right)-\varphi_{n_{0}+1}\left(z_{l}\right)\right|+|| \varphi-\varphi_{n_{0}+1}||_{\text {op }}|| z_{l}\right\|_{\mathfrak{H}} \\
& \leq 2 M\left\|\varphi-\varphi_{n_{0}+1}\right\|_{\text {op }}+\left|\varphi_{n_{0}+1}\left(z_{k}\right)-\varphi_{n_{0}+1}\left(z_{l}\right)\right| \\
& <\frac{\varepsilon}{2}+\frac{\varepsilon}{2}=\varepsilon \quad \forall k, l>k_{0}
\end{aligned}
$$

Como $\varepsilon>0$ é arbitrário, provamos que $\left(\varphi\left(z_{k}\right)\right)_{k \in \mathbb{N}}$ é de Cauchy, e logo convergente. Concluído mais uma etapa, fica bem definido o funcional linear

$$
\Theta\left(u^{*}\right) \doteq \lim _{k \rightarrow \infty} u^{*}\left(z_{k}\right), \quad u \in \mathfrak{H}
$$

Note que para cada $u \in \mathfrak{H}$ que fixamos, vale

$$
\left|u^{*}\left(z_{k}\right)\right| \leq\left\|u^{*}\right\|_{\text {op }}|| z_{k}\left\|_{\mathfrak{H}} \leq M\right\| u^{*} \|_{\text {op }} \quad \forall k \in \mathbb{N}
$$

e no limite

$$
\left|\Theta\left(u^{*}\right)\right|=\lim _{k \rightarrow \infty}\left|u^{*}\left(z_{k}\right)\right| \leq\left. M|| u^{*}\right|_{\text {op }}
$$

ou seja, $\Theta$ é um elemento genuíno do bidual topológico de $\mathfrak{H}$. Como todo espaço de Hilbert é reflexivo (isto é, $\varkappa$ é sobrejetora), existe $z \in \mathfrak{H}$ de modo que $\varkappa(z)=\Theta$, e assim

$$
\lim _{k \rightarrow \infty} u^{*}\left(z_{k}\right)=\Theta\left(u^{*}\right)=\varkappa(z)\left(u^{*}\right)=u^{*}(z) \quad \forall u \in \mathfrak{H}
$$

ou seja, $z_{k} \stackrel{\text { w. }}{\longrightarrow} z$.

Definição 19 (Sequência de Weyl). Uma sequência de vetores $\left(\psi_{n}\right)_{n \in \mathbb{N}}$ em um espaço de Hilbert $\mathfrak{H}$ é dita ser de Weyl para $A \in \mathcal{B}(\mathfrak{H})$ e $\lambda \in \mathbb{C}$ quando cumpre as seguintes condições:
a) $\psi_{n} \in \mathbb{S}^{1}(\mathfrak{H}) \quad \forall n \in \mathbb{N}$
b) $\mathrm{w}-\lim _{n \rightarrow \infty} \psi_{n}=0$
c) $\lim _{n \rightarrow \infty}\left\|(A-\lambda \mathbb{1}) \psi_{n}\right\|_{\mathfrak{H}}=0$

Recorde que o espectro de um operador limitado $T$ agindo em um espaço de Banach $X$ é dividido em duas unidades básicas, sendo a primeira delas

$$
\sigma_{\mathrm{d}}(T) \doteq\left\{\lambda \in \mathbb{C}: \lambda \text { é um autovalor isolado de } T, \mathrm{~m}_{g}(\lambda ; T)<+\infty\right\}
$$

designada como espectro discreto, e o que sobra

$$
\sigma_{\mathrm{ess}}(T) \doteq \sigma(T) \backslash \sigma_{\mathrm{d}}(T)
$$


denominado por espectro essencial. Entendemos por isolado no sentido topológico, ou seja, existe uma bola aberta (no plano complexo) de centro $\lambda$ que o isola completamente dos demais pontos do espectro de $T$.

Definição 20 (Espectro de Weyl). Seja $\mathfrak{H}$ um espaço de Hilbert. O espectro de Weyl de um operador $T \in \mathcal{B}(\mathfrak{H})$ é definido como

$$
\sigma_{\mathrm{w}}(T) \doteq\{\lambda \in \mathbb{C} \text { : existe uma sequência de Weyl para } T \text { e } \lambda\}
$$

A questão que prevalece é saber onde se localiza essa terceira instância do espectro, e disso que trata o critério de Weyl à seguir. Mas primeiro, vejamos com que simplicidade o espectro de Weyl de uma soma direta de operadores autoadjuntos se relaciona com o das partes.

Proposição 21. Seja $A$ e $B$ operadores limitados e autoadjuntos agindo nos espaços de Hilbert $\mathfrak{H} e$ $\mathcal{H}$, respectivamente. São verdadeiras as seguintes afirmações:
a) $\sigma_{\mathrm{w}}(A \oplus B)=\sigma_{\mathrm{w}}(A) \cup \sigma_{\mathrm{w}}(B)$
b) $\sigma(A \oplus B)=\sigma(A) \cup \sigma(B)$

Demonstração. Se $\left(\psi_{n}, \varphi_{n}\right)_{n \in \mathbb{N}}$ é uma sequência de Weyl para $A \oplus B$ e $\lambda$, então

$$
\left\|(A-\lambda \mathbb{1}) \psi_{n}\right\|_{\mathfrak{H}}+\left\|(B-\lambda \mathbb{1}) \varphi_{n}\right\|_{\mathcal{H}}=\left\|(A \oplus B-\lambda \mathbb{1})\left(\psi_{n}, \varphi_{n}\right)\right\|_{\mathfrak{H} \oplus \mathcal{H}} \longrightarrow 0
$$

o que obriga cada parcela tender à zero individualmente. Se $\pi$ denota a projeção na primeira coordenada, efetua-se

$$
\left(\psi_{n}, \varphi_{n}\right) \stackrel{\text { w. }}{\longrightarrow}(0,0) \Longrightarrow \psi_{n}=\pi\left(\psi_{n}, \varphi_{n}\right) \stackrel{\text { w. }}{\longrightarrow} 0
$$

por conta da (P.17), e através de um raciocínio análogo conclui-se $\varphi_{n} \stackrel{\text { w. }}{\longrightarrow} 0$. No tocante a normalização, sabe-se

$$
1=\left\|\left(\psi_{n}, \varphi_{n}\right)\right\|_{\mathfrak{H} \oplus \mathcal{H}}^{2}=\left\|\psi_{n}\right\|_{\mathfrak{H}}^{2}+\left\|\varphi_{n}\right\|_{\mathcal{H}}^{2} \quad \forall n \in \mathbb{N}
$$

Assim, se os $\psi_{n}$ (respec. $\varphi_{n}$ ) possui uma subsequência formada única e exclusivamente pelo vetor nulo, a subsequência dos $\varphi_{n}$ (respec. $\psi_{n}$ ) correspondente é constituída de vetores normalizados, e com isso Weyl para $B$ (respec. $A)$ e $\lambda$.

No pior dos casos, ambas as sequências não se anulam a partir de um dado momento, mas pelo fato da norma das $\psi_{n}$ estar restrita ao intervalo $[0,1]$, existe uma subsequência para o qual $\left(\left\|\psi_{n_{k}}\right\|_{\mathfrak{H}}\right)_{k \in \mathbb{N}}$, e portanto $\left(\left\|\varphi_{n_{k}}\right\|_{\mathfrak{H}}\right)_{k \in \mathbb{N}}$, converge. Com base na relação acima, sabe-se ainda que ao menos um desses limites é diferente de zero. Supondo que seja o da primeira sequência, $\left(\left\|\psi_{n_{k}}\right\|_{\mathfrak{H}}^{-1} \psi_{n_{k}}\right)_{k>k_{0}}$ é Weyl para $A$ e $\lambda$.

A recíproca é evidente, pois se $\left(\psi_{n}\right)_{n \in \mathbb{N}}$ for Weyl pra $A$ (respec. $\left.B\right)$ e $\lambda,\left(\psi_{n}, 0\right)_{n \in \mathbb{N}}\left(\right.$ respec. $\left.\left(0, \psi_{n}\right)_{n \in \mathbb{N}}\right)$ é Weyl pra $A \oplus B$ e $\lambda$. Graças ao (T.13), a prova de b) segue do mesmo modo, só que ignorando os trechos relativos à convergência fraca.

Lema 22. Seja $\mathfrak{H}$ um espaço de Hilbert e $A \in \mathcal{B}(\mathfrak{H})$ autoadjunto. Se $\lambda$ é um ponto isolado de $\sigma(A)$, então $A-\lambda \mathbb{1}\left\lceil\mathfrak{H}_{\lambda}(A)^{\perp}\right.$ é inversível. 
Demonstração. Pelo teorema da decomposição ortogonal, sabemos que $\mathfrak{H}$ se decompõe na soma direta

$$
\mathfrak{H}=\mathfrak{H}_{\lambda}(A) \oplus \mathfrak{H}_{\lambda}(A)^{\perp}
$$

onde cada um desses espaços é $A$-invariente ${ }^{18}$. Posto isto,

$$
A=\left(A \lceil \mathfrak { H } _ { \lambda } ( A ) ) \oplus \left(A\left\lceil\mathfrak{H}_{\lambda}(A)^{\perp}\right)\right.\right.
$$

e por consequência

$$
\sigma(A) \stackrel{19}{=} \sigma\left(A \lceil \mathfrak { H } _ { \lambda } ( A ) ) \cup \sigma \left(A\left\lceil\mathfrak{H}_{\lambda}(A)^{\perp}\right)\right.\right.
$$

Claro que $\lambda$ não é um autovalor de $B \doteq A\left\lceil\mathfrak{H}_{\lambda}(A)^{\perp}\right.$, afinal os autovetores de $A$ associados a esse valor foram banidos do espaço. Mas então $\lambda \in \mathcal{R}(B)$, caso contrário a relação acima diria que $\lambda$ é um ponto isolado de $\sigma(B)$, e portanto um autovalor de $B$, vide (C.12).

Teorema 23 (Critério de Weyl). Em um espaço de Hilbert separável $\mathfrak{H}$, o espectro de todo operador $A \in \mathcal{B}(\mathfrak{H})$ autoadjunto se decompõe na união disjunta de seu espectro de Weyl com seu espectro discreto. Simbolicamente,

$$
\sigma(A)=\sigma_{\mathrm{d}}(A) \dot{\cup} \sigma_{\mathrm{w}}(A)
$$

ou ainda, $\sigma_{\mathrm{ess}}(A)=\sigma_{\mathrm{w}}(A)$.

Demonstração. Suponha que $\lambda \in \sigma_{\text {ess }}(A)$. O (T.13) garante que é possível arranjar uma sequência $\left(\psi_{n}\right)_{n \in \mathbb{N}}$ em $\mathbb{S}^{1}(\mathfrak{H})$ de modo a

$$
\lim _{n}\left\|(A-\lambda \mathbb{1}) \psi_{n}\right\|_{\mathfrak{H}}=0
$$

e que por passagem a uma subsequência, se necessário, também podemos assumir que converge fracamente para um determinado $\psi \in \mathfrak{H}$. Se $\psi=0$, já temos $\lambda \in \sigma_{\mathrm{w}}(A)$. Caso contrário,

$$
0 \leq\left|\left\langle\varphi,(A-\lambda \mathbb{1}) \psi_{n}\right\rangle_{\mathfrak{H}}\right| \leq\left\|\left.\varphi\right|_{\mathfrak{H}}||(A-\lambda \mathbb{1}) \psi_{n}\right\|_{\mathfrak{H}} \longrightarrow 0 \quad \forall \varphi \in \mathfrak{H}
$$

e por consequência

$$
(A-\lambda \mathbb{1}) \psi_{n} \stackrel{\text { w. }}{\longrightarrow} 0
$$

Em compensação, a (P.17) afirma

$$
(A-\lambda \mathbb{1}) \psi_{n} \stackrel{\text { w. }}{\longrightarrow}(A-\lambda \mathbb{1}) \psi
$$

e por unicidade $A \psi=\lambda \psi$, ou seja, $\lambda$ é um autovalor de $A$, que por se tratar de um ponto espectral essencial, ou possui multiplicidade (geométrica) infinita, ou é ponto de acumulação de $\sigma(A)$.

No primeiro caso, qualquer base ortonormal de $\mathfrak{H}_{\lambda}(A)$ define prontamente uma sequência de Weyl para $A$ e $\lambda$, e com isso $\lambda \in \sigma_{\mathrm{w}}(A)$. Já no segundo, considere uma sequência $\left(\lambda_{j}\right)_{j \in \mathbb{N}}$ em $\sigma(A) \backslash\{\lambda\}$ cujos elementos são dois-a-dois distintos e convergem à $\lambda$. Caso haja uma subsequência $\left(\lambda_{j_{k}}\right)_{k \in \mathbb{N}}$ constituída integralmente de autovalores de $A$, a sequência $\left(\varphi_{k}\right)_{k \in \mathbb{N}}$ formada por seus autovetores correspondentes e normalizados configura uma sequência de Weyl para $A$ e $\lambda$, e de novo $\lambda \in \sigma_{\mathrm{w}}(A)$. De fato,

\footnotetext{
${ }^{18}$ De modo geral, se $T \in \mathcal{B}(\mathfrak{H})$ e $\mathcal{S} \leq \mathfrak{H}$ é $T$-invariante, então $\mathcal{S}^{\perp}$ é $T^{*}$-invariante.

${ }^{19}$ Embora tenhamos provado a (P.21) para uma soma direta externa (e não interna) de operadores, essas noções estão conectadas por uma conjugação unitária.
} 


$$
\left\|(A-\lambda \mathbb{1}) \varphi_{k}\right\|_{\mathfrak{H}}=\left\|\left(A-\lambda_{j_{k}} \mathbb{1}\right) \varphi_{k}+\left(\lambda_{j_{k}}-\lambda\right) \varphi_{k}\right\|_{\mathfrak{H}}=\left|\lambda_{j_{k}}-\lambda\right| \cdot\left\|\varphi_{k}\right\|_{\mathfrak{H}}=\left|\lambda_{j_{k}}-\lambda\right| \longrightarrow 0
$$

além do que $\varphi_{k} \stackrel{\text { w. }}{\longrightarrow} 0$, visto que autovalores distintos de um mesmo operador autoadjunto estão associados a autovetores ortogonais.

Agora, se a partir de um certo $j_{0} \in \mathbb{N}$ os $\lambda_{j}$ não for autovalor de $A$, podemos reargumentar tudo de novo para cada $\lambda_{j} \in \sigma_{\text {ess }}(A)$ com $j>j_{0}$, obtendo em cada caso uma sequência $\left(\phi_{j, k}\right)_{k \in \mathbb{N}}$ em $\mathbb{S}^{1}(\mathfrak{H})$ verificando o limite

$$
\lim _{k}\left\|\left(A-\lambda_{j} \mathbb{1}\right) \phi_{j, k}\right\|_{\mathfrak{H}}=0
$$

e convergindo fracamente para o vetor nulo, pois agora sabemos de antemão que $\lambda_{j}$ não é autovalor. Pelo argumento da sequência diagonal, extraímos então uma sequência de Weyl para $A$ e $\lambda$.

Reciprocamente, suponha $\lambda \in \sigma_{\mathrm{w}}(A) \cap \sigma_{\mathrm{d}}(A)$, e seja $\left(\psi_{n}\right)_{n \in \mathbb{N}}$ uma sequência de Weyl correspondente. Seja também $\left\{\varphi_{1}, \ldots, \varphi_{l}\right\}$ uma base ortonormal de $\mathfrak{H}_{\lambda}(A)$ e denote com $P$ o projetor ortogonal de $\mathfrak{H}$ em $\mathfrak{H}_{\lambda}(A)^{\perp}$. Temos

$$
\left\|P \psi_{n}\right\|_{\mathfrak{H}}^{2}=1-\sum_{k=1}^{l}\left|\left\langle\varphi_{k}, \psi_{n}\right\rangle_{\mathfrak{H}}\right|^{2} \longrightarrow 1
$$

por causa que $\psi_{n} \stackrel{\text { w. }}{\longrightarrow} 0$, e além disso

$$
\left\|(A-\lambda \mathbb{1}) P \psi_{n}\right\|_{\mathfrak{H}}=\left\|(A-\lambda \mathbb{1}) \psi_{n}\right\|_{\mathfrak{H}} \longrightarrow 0
$$

Pois sendo $B \doteq(A-\lambda \mathbb{1}) \uparrow \mathfrak{H}_{\lambda}(A)^{\perp}$ inversível,

$$
1 \longleftarrow\left\|P \psi_{n}\right\|_{\mathfrak{H}}^{2} \leq\left\|B^{-1} B P \psi_{n}\right\|_{\mathfrak{H}}^{2} \leq\left\|B^{-1}\right\|_{\text {op }}\left\|(A-\lambda \mathbb{1}) P \psi_{n}\right\|_{\mathfrak{H}} \longrightarrow 0
$$

o que é um absurdo, visto que a teoria geral de sequências numéricas prevê a manutenção da desigualdade no limite. Logo não podemos supor que espectro discreto e o de Weyl possua algum ponto em comum. Também sabemos pelo (T.13) que $\sigma_{\mathrm{w}}(A) \subseteq \sigma(A)$, e daí $\sigma_{\mathrm{w}}(A) \subseteq \sigma_{\text {ess }}(A)$.

Com base no critério de Weyl, é possível provar que a parte essencial do espectro não se altera quando o perturbamos por meio de um segundo operador autoadjunto e compacto. Eis o enunciado preciso:

Teorema 24. Seja $\mathfrak{H}$ um espaço de Hilbert separável e $A, K \in \mathcal{B}(\mathfrak{H})$ autoadjuntos. Se $K$ for compacto, então

$$
\sigma_{\mathrm{ess}}(A)=\sigma_{\mathrm{ess}}(A+K)
$$

Demonstração. Se $\left(\psi_{n}\right)_{n \in \mathbb{N}}$ é uma sequência de Weyl para $A$ e $\lambda$, computamos

$$
\left\|(A+K-\lambda \mathbb{1}) \psi_{n}\right\|_{\mathfrak{H}}=\left\|(A-\lambda \mathbb{1}) \psi_{n}+K \psi_{n}\right\|_{\mathfrak{H}} \leq\left\|(A-\lambda \mathbb{1}) \psi_{n}\right\|_{\mathfrak{H}}+\left\|K \psi_{n}\right\|_{\mathfrak{H}} \longrightarrow 0
$$

e caso seja Weyl para $A+K$ e $\lambda$, efetuamos

$$
\left\|(A-\lambda \mathbb{1}) \psi_{n}\right\|_{\mathfrak{H}}=\left\|(A+K-\lambda \mathbb{1}) \psi_{n}-K \psi_{n}\right\|_{\mathfrak{H}} \leq\left\|(A+K-\lambda \mathbb{1}) \psi_{n}\right\|_{\mathfrak{H}}+\left\|K \psi_{n}\right\|_{\mathfrak{H}} \longrightarrow 0
$$

Em qualquer casos, $\psi_{n} \stackrel{\text { w. }}{\longrightarrow} 0 \Longrightarrow K \psi_{n} \longrightarrow 0$ por conta da compacidade de $K$, vide (P.17). 


\subsection{Princípio de Birman-Schwinger}

"O que pode ser afirmado sem provas, pode ser descartado sem provas."

Christopher Hitchens ${ }^{20}$

Existem inúmeras formulações do princípio de Birman-Schwinger, eis aqui uma delas:

Teorema 25 (Birman-Schwinger). Seja $X$ um $K$-espaço vetorial, $H_{0}, V \in \mathrm{L}(\mathcal{X})$ e $\lambda \in K$ de forma que $H_{0}-\lambda \mathbb{1}$ seja inversivel. Assuma ainda que $V$ seja não nulo e admita uma raiz quadrada $R$. Então, $\lambda$ é autovalor de $H_{0}-V$ se, e somente se, 1 for autovalor de

$$
\mathrm{B}(\lambda) \doteq R\left(H_{0}-\lambda \mathbb{1}\right)^{-1} R
$$

Em caso afirmativo,

$$
\mathrm{m}_{g}\left(\lambda ; H_{0}-V\right)=\mathrm{m}_{g}(1 ; \mathrm{B}(\lambda))
$$

Demonstração. Suponha que $\lambda$ seja um autovalor de $H_{0}-V$ e tomemos $\left\{\varphi_{i}\right\}_{i \in I}$ uma base de seu correspondente autoespaço. Pondo $\psi_{i} \doteq R \varphi_{i}$, tem-se

$$
\left(H_{0}-\lambda \mathbb{1}\right) \varphi_{i}=\left(H_{0}-V\right) \varphi_{i}+(V-\lambda \mathbb{1}) \varphi_{i}=\lambda \varphi_{i}+(V-\lambda \mathbb{1}) \varphi_{i}=V \varphi_{i}
$$

e $\log O$

$$
\varphi_{i}=\left(H_{0}-\lambda \mathbb{1}\right)^{-1} V \varphi_{i}=\left(H_{0}-\lambda \mathbb{1}\right)^{-1} R \psi_{i}
$$

seja qual for $i \in I$. Dado que transformações lineares enviam vetores LD em vetores LD, vemos que $\left\{\psi_{i}\right\}_{i \in I}$ só pode ser LI. Aplicando $R$ na equação acima, também deduzimos

$$
\mathrm{B}(\lambda) \psi_{i}=R \varphi_{i}=\psi_{i} \quad \forall i \in I
$$

o que significa que $\left\{\psi_{i}\right\}_{i \in I}$ está contido no autoespaço de $\mathrm{B}(\lambda)$ associado ao valor 1 , e em suma

$$
\mathrm{m}_{g}(1 ; \mathrm{B}(\lambda)) \geq \mathrm{m}_{g}\left(\lambda ; H_{0}-V\right)
$$

A recíproca é análoga: Se $\left\{\phi_{j}\right\}_{j \in J}$ configura uma base do autoespaço de $\mathrm{B}(\lambda)$ associado ao valor 1 , ponha $\eta_{j} \doteq\left(H_{0}-\lambda \mathbb{1}\right)^{-1} R \phi_{j}$ que pela mesma razão de outrora

$$
\phi_{j}=\mathrm{B}(\lambda) \phi_{j}=R \eta_{j}
$$

e

$$
\left(H_{0}-V\right) \eta_{j}=\left(H_{0}-\lambda \mathbb{1}\right) \eta_{j}+(\lambda \mathbb{1}-V) \eta_{j}=R \phi_{j}+(\lambda \mathbb{1}-V) \eta_{j}=\lambda \eta_{j}
$$

garantem que $\left\{\eta_{j}\right\}_{j \in J}$ é LI e está contido no autoespaço de $H_{0}-V$ associado ao valor $\lambda$. Em particular

$$
\mathrm{m}_{g}\left(\lambda ; H_{0}-V\right) \geq \mathrm{m}_{g}(1 ; \mathrm{B}(\lambda))
$$

\footnotetext{
${ }^{20}$ Christopher Eric Hitchens, $\nabla(13 / 04 / 1949)-\dagger(15 / 12 / 2011)$
} 
e o teorema está provado.

Apesar de nossa exposição puramente algébrica, este princípio se encontra bastante difundido no seguinte contexto:

Definição 26 (Operador de Birman-Schwinger). Seja $\mathfrak{H}$ um espaço de Hilbert e $H_{0}, V \in \mathcal{B}(\mathfrak{H})$ com $V \geq 0$. Dizemos que

$$
\mathrm{B}_{H_{0}, V}(\lambda) \doteq V^{1 / 2} \mathcal{R}\left(\lambda ; H_{0}\right) V^{1 / 2}
$$

é o operador de Birman Schwinger associado ao par $\left(H_{0}, V\right)$ no ponto $\lambda \in \mathcal{R}\left(H_{0}\right)$.

Com isso encerramos o conteúdo programado para este capítulo. 


\section{Capítulo 2}

\section{Integral Direta de Von Neumann}

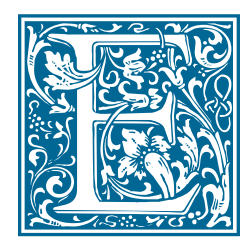

M MATEmática e, principalmente na análise funcional, integral direta trata-se de uma tentativa bem sucedida de se generalizar o conceito de soma direta que usualmente concebemos. Este conceito foi pioneiramente introduzido em 1949 por John von Neumann em um de seus papers da série "On Rings of Operators", e com o tempo vem sendo empregado em diversas áreas da física matemática como a teoria quântica de campos, mecânica quântica de muitas partículas, física de corpos rígidos, etc; ainda que desconhecida pela maior parte da comunidade científica.

Noções Gerais e Teoremas: Mensurabilidade Fraca, Teorema de Riesz-Fischer, Mensurabilidade na Topologia Fraca de Operador, Integral Direta de um Espaço de Hilbert, Integral Direta de Operadores, Teorema da Convergência Pontual, Cálculo Funcional Boreliano e Espectro de um Operador Decomponível, Espectro e Espaço Espectral Absolutamente Contínuo de um Operador de Multiplicação.

\subsection{Espaços L ${ }^{p}$ Generalizados}

"Faça as coisas o mais simples que você puder, porém não se restrinja às mais simples."

\section{Albert Einstein ${ }^{1}$}

Definição 27 (Função Fracamente Mensurável). Seja y um $\mathbb{K}$-espaço vetorial normado e $(X, \Omega)$ um espaço mensurável. Uma função $f: X \longrightarrow y$ diz-se fracamente mensurável quando

$$
\ell \circ f: X \longrightarrow \mathbb{K}
$$

for $\Omega$-mensurável (no sentido usual), qualquer que seja $\ell \in \mathrm{y}^{*}$.

O conjunto $\mathcal{W}(X, y)$ de tais aplicações forma um $\Sigma(\Omega)$-módulo quando munido das operações ponto-aponto de funções porque se $f, g \in \mathcal{W}(X, y)$ e $\alpha \in \Sigma(\Omega)$, também são fracamente mensuráveis as seguintes aplicações:

- $f+g(x) \doteq f(x)+g(x)$, já que para cada $\ell \in y^{*}, \ell \circ(f+g)=\ell \circ f+\ell \circ g$ é soma pontual de duas funções (por hipótese) $\Omega$-mensuráveis, e logo $\Omega$-mensurável.

${ }^{1}$ Albert Einstein, $\odot(14 / 03 / 1879)-\dagger(18 / 04 / 1955)$ 
- $\alpha f(x) \doteq \alpha(x) f(x)$, já que para cada $\ell \in \mathrm{y}^{*}, \ell \circ(\alpha f)=\alpha \cdot(\ell \circ f)$ resulta do produto ponto-a-ponto de duas funções (por hipótese) $\Omega$-mensuráveis, portanto $\Omega$-mensurável.

Esta nova noção de mensurabilidade é estável com relação ao limite pontual, composição com operadores limitados e justaposição. Com precisão:

- Se $\left(f_{n}\right)_{n \in \mathbb{N}}$ é uma sequência em $\mathcal{W}(X, \boldsymbol{y})$ tal que $f_{n} \stackrel{p \text {. }}{\longrightarrow} f$, a continuidade de todo funcional $\ell \in \mathcal{y}^{*}$ implica $\ell \circ f_{n} \stackrel{p .}{\longrightarrow} \ell \circ f$, o que expressa essa última como limite pontual de uma sequência de funções (por hipótese) $\Omega$-mensuráveis, e logo $\Omega$-mensurável.

- Se $z$ denota um segundo $\mathbb{K}$-espaço vetorial e $T \in \mathcal{B}(y, z)$, pela associatividade da composição

$$
\ell \circ(T \circ f)=(\ell \circ T) \circ f \in \Sigma(\Omega)
$$

já que $\ell \circ T \in \mathrm{y}^{*}$, seja qual for $\ell \in z^{*}$.

- Se $\iota_{y}$ e $\iota_{z}$ denotam as inclusões do espaço indicado em $y \times z$, computamos

$$
(f, g)=\iota_{y} \circ f+\iota_{z} \circ g
$$

o que exprime $(f, g)$ como soma de duas funções fracamente mensuráveis em $y \times z$, haja visto o fato anterior.

- Se $\pi_{y}$ e $\pi_{z}$ denotam as projeções de $y \times z$ no espaço indicado, escrevemos

$$
f=\pi_{y} \circ(f, g) \quad, \quad g=\pi_{z} \circ(f, g)
$$

e o penúltimo item se aplica novamente, ou seja, a mensurabilidade (no sentido fraco) do par $(f, g)$ também garante a mensurabilidade de suas coordenadas de maneira recíproca.

Levando em conta apenas as funções de $\Sigma(\Omega)$ que são constantes, também provamos que $\mathcal{W}(X, y)$ é um $\mathbb{K}$-espaço vetorial, cujo vetor nulo é a função constante nula.

Em virtude do teorema de representação de Riesz para um funcional linear limitado, uma aplicação $f$, definida em $X$ e assumindo valores em um espaço de Hilbert $\mathfrak{H}$ é fracamente mensurável quando

$$
X \ni x \longmapsto\langle y, f(x)\rangle_{\mathfrak{H}} \in \mathbb{C}
$$

for $\Omega$-mensurável para todo $y \in \mathfrak{H}$. Se exigirmos de $\mathfrak{H}$ o terceiro axioma da enumerabilidade, então dada uma segunda $g \in \mathcal{W}(X, \mathfrak{H})$, são $\Omega$-mensuráveis as aplicações:

- $X \ni x \longmapsto\|f(x)\|_{\mathfrak{H}} \in \mathbb{R}$, pois de acordo com a identidade de Parseval, é limite pontual de uma sequência de funções (por hipótese) $\Omega$-mensuráveis.

- $X \ni x \longmapsto\langle f(x), g(x)\rangle_{\mathfrak{H}} \in \mathbb{C}$, já que a identidade de polarização o exprime como combinação linear de funções $\Omega$-mensuráveis.

É por isso que se dotamos $(X, \Omega)$ de uma medida $\mu$, faz sentido considerar o conjunto

$$
\mathcal{L}_{\mathfrak{H}}^{p}(X, \Omega, \mu) \doteq\left\{f \in \mathcal{W}(X, \mathfrak{H}): \int_{X}\|f(x)\|_{\mathfrak{H}}^{p} \mathrm{~d} \mu(x)<+\infty\right\}, \quad 1 \leq p<+\infty
$$

do qual facilmente nos convencemos ser um $\mathbb{C}$-subespaço vetorial de $\mathcal{W}(X, \mathfrak{H})$, uma vez conhecidos as propriedades e resultados básicos para espaços $L^{p}$ ordinários. Pela mesma razão, 


$$
Q(f) \doteq\left(\int_{X}\|f(x)\|_{\mathfrak{H}}^{p} \mathrm{~d} \mu(x)\right)^{\frac{1}{p}}, \quad f \in \mathcal{L}_{\mathfrak{H}}^{p}(X)
$$

define uma seminorma em $\mathcal{L}_{\mathfrak{H}}^{p}(X)$, e

$$
\mathrm{L}_{\mathfrak{H}}^{p}(X, \Omega, \mu) \doteq \frac{\mathcal{L}_{\mathfrak{H}}^{p}(X, \Omega, \mu)}{\{f: Q(f)=0\}}
$$

por sua vez, um $\mathbb{C}$-espaço vetorial normado, quando munido das operações usuais para classes de equivalência

- $(f+\mathcal{N})+(g+\mathcal{N})=(f+g)+\mathcal{N}$

(Soma)

- $\alpha(f+\mathcal{N})=(\alpha f)+\mathcal{N}$

(Multiplicação por Escalar)

- $\|f+\mathcal{N}\|_{p, \mathfrak{H}} \doteq Q(f)$

(Norma)

conforme é previsto pela teoria geral dos espaços quocientes. Usamos a notação $\mathcal{N} \doteq Q^{-1}(0)$.

Vale lembra que, por definição, duas funções $f$ e $g$ induzem a mesma classe de equivalência se $f-g \in \mathcal{N}$, ou seja, se $Q(f-g)=0$, e portanto quando coincidem em $\mu$-quase toda parte.

Teorema 28 (Riesz-Fischer). Seja $\mathfrak{H}$ um espaço de Hilbert separável, $(X, \Omega, \mu)$ um espaço de medida e $p \in[1,+\infty)$. Então, $\mathrm{L}_{\mathfrak{H}}^{p}(X, \Omega, \mu)$ forma um espaço de Banach.

Demonstração. Seja, pois, $\left(f_{n}+\mathcal{N}\right)_{n \in \mathbb{N}}$ uma sequência em $\mathrm{L}_{\mathfrak{H}}^{p}$ que converge absolutamente, isto é,

$$
\sum_{1}^{\infty}\left\|f_{n}+\mathcal{N}\right\|_{p, \mathfrak{H}}=B<+\infty
$$

Pondo $G_{n} \doteq\left\|f_{n}(\cdot)\right\|_{\mathfrak{H}} \in \mathrm{L}^{p}(X)$, veja em primeiro lugar que $G \doteq \sum_{1}^{\infty} G_{n}$ é limite pontual de uma sequência de funções $\Omega$-mensuráveis, e portanto ela própria deve ser $\Omega$-mensurável. Além disso

$$
\left\|G_{n}\right\|_{p}=Q\left(f_{n}\right)=\left\|f_{n}+\mathcal{N}\right\|_{p, \mathfrak{H}} \forall n \in \mathbb{N}
$$

o que nos permite obter a seguinte majoração

$$
\left(\int_{X}\left|\sum_{1}^{k} G_{n}\right|^{p} \mathrm{~d} \mu\right)^{\frac{1}{p}}=\left\|\sum_{1}^{k} G_{n}\right\|_{p} \leq \sum_{1}^{k}\left\|G_{n}\right\|_{p} \leq \sum_{1}^{\infty}\left\|G_{n}\right\|_{p}=B<+\infty \quad \forall k \in \mathbb{N}
$$

mantida inclusive no limite quando $k \rightarrow \infty$, caso ele exista. Oras, segue da positividade das $G_{n}$ que $\left(\left|\sum_{1}^{k} G_{n}\right|^{p}\right)_{k \in \mathbb{N}}$ é crescente, e pelo Teorema da Convergência Monótona

$$
\int_{X}|G|^{p} \mathrm{~d} \mu=\lim _{k} \int_{X}\left|\sum_{1}^{k} G_{n}\right|^{p} \mathrm{~d} \mu \leq B^{p}<+\infty
$$

que além de consagrar $G$ como um elemento legítimo de $\mathrm{L}^{p}(X)$, nos informa que a mesma deve ser finita em $\mu$-quase toda parte. Voltando em sua definição, concluímos que em $\mu$-quase toda parte $\sum_{1}^{\infty} f_{n}$ converge absolutamente, e portanto simplesmente, uma vez que $\mathfrak{H}$ é Banach. Definindo o conjunto de medida total

$$
E \doteq\left\{x \in X: \sum_{1}^{\infty}\left\|f_{n}(x)\right\|_{\mathfrak{H}}<+\infty\right\}
$$

ponha $F=\sum_{1}^{\infty} f_{n}$ em $E$ e anule-a no complementar. Para todo $y \in \mathfrak{H}$, a restrição de $y^{*} \circ F$ em $X \backslash E$ é $(\Omega \nmid X \backslash E)$-mensurável por que é constante (igual à 0$)$ e restrição de $y^{*} \circ F$ em $E$ é $(\Omega\lceil E$ )-mensurável por que se exprime como limite pontual de uma sequência de funções $(\Omega \uparrow E)$-mensuráveis. Isso prova que $y^{*} \circ F$ é $\Omega$-mensurável, e logo $F \in \mathcal{W}(X, \mathfrak{H})$. Por conseguinte, computamos 


$$
\left\|\sum_{1}^{n} f_{k}(x)\right\|_{\mathfrak{H}} \leq \sum_{1}^{n}\left\|f_{k}(x)\right\|_{\mathfrak{H}} \leq \sum_{1}^{\infty}\left\|f_{k}(x)\right\|_{\mathfrak{H}}=G(x) \quad \forall x \in E, n \in \mathbb{N}
$$

que sob as diretrizes da continuidade da norma de $\mathfrak{H}$, nos confere a desigualdade

$$
\|F(x)\|_{\mathfrak{H}}=\lim _{n}\left\|\sum_{1}^{n} f_{k}(x)\right\|_{\mathfrak{H}} \leq G(x)
$$

válida inclusive fora de $E$, e portanto em toda extensão de $X$. Disso segue $F \in \mathcal{L}_{\mathfrak{H}}^{p}(X)$ e finaliza a construção do candidato

$$
\sum_{1}^{\infty}\left(f_{k}+\mathcal{N}\right) \stackrel{!}{=} F+\mathcal{N}
$$

na norma $\|\cdot\|_{p, \mathfrak{H}} \cdot$ Com efeito,

$$
\left\|F(x)-\sum_{1}^{n} f_{k}(x)\right\|_{\mathfrak{H}} \leq\|F(x)\|_{\mathfrak{H}}+\sum_{1}^{n}\left\|f_{k}(x)\right\|_{\mathfrak{H}} \leq 2 G(x) \quad \forall x \in E
$$

donde

$$
\left\|F(\cdot)-\sum_{1}^{n} f_{k}(\cdot)\right\|_{\mathfrak{H}}^{p} \leq(2 G)^{p} \in \mathrm{L}^{1}(X) \quad \mu \text {-q.t.p }
$$

o que nos coloca em posição de aplicar o teorema da convergência dominada, e assim deduzir

$$
\begin{aligned}
\lim _{n \rightarrow \infty}\left\|(F+\mathcal{N})-\sum_{k=1}^{n}\left(f_{k}+\mathcal{N}\right)\right\|_{p, \mathfrak{H}}^{p} & =\lim _{n \rightarrow \infty}\left\|\left(F-\sum_{k=1}^{n} f_{k}\right)+\mathcal{N}\right\|_{p, \mathfrak{H}}^{p}= \\
& =\lim _{n \rightarrow \infty} Q\left(F-\sum_{k=1}^{n} f_{k}\right)^{p}=\lim _{n \rightarrow \infty} \int_{X}\left\|F(x)-\sum_{k=1}^{n} f_{k}(x)\right\|_{\mathfrak{H}}^{p} \mathrm{~d} \mu(x)=0
\end{aligned}
$$

Com isso se encerra a demonstração.

Como de praxe no contexto dos espaços $\mathrm{L}^{p}$, nos permitimos um pequeno abuso de linguagem ao denotar os membros de $L_{\mathfrak{H}}^{p}$ simplesmente por $f$ (abandonando a notação de classes $f+\mathcal{N}$ ) e agindo de acordo, ou seja, entendendo que as propriedades de mensurabilidade (no sentido fraco) e integrabilidade correspondem aos elementos de $L_{\mathfrak{H}}^{p}$.

Definição 29 (Função Mensurável na Topologia Fraca de Operador). Seja $\mathfrak{H}$ um espaço de Hilbert separável e $(X, \Omega)$ um espaço mensurável. Uma aplicação $A: X \longrightarrow \mathcal{B}(\mathfrak{H})$ diz-se mensurável na topologia fraca de operador quando cumpre a seguinte condição:

$$
A(\cdot) \psi \in \mathcal{W}(X, \mathfrak{H}) \quad \forall \psi \in \mathfrak{H}
$$

Do que sabemos à respeito de $\mathcal{W}(X, \mathfrak{H})$, fica óbvio que o conjunto $\mathfrak{A}(X, \mathfrak{H})$ de tais aplicações forma uma $\Sigma(\Omega)$-álgebra involutiva associativa e com unidade se munido das seguintes operações ponto-a-ponto:

- $A+B(x) \doteq A(x)+B(x)$

(Soma)

- $\alpha A(x) \doteq \alpha(x) A(x)$

(Multiplicação por Escalar)

- $A^{*}(x) \doteq A(x)^{*}$

(Involução)

- $A B(x) \doteq A(x) B(x)$

(Produto) 
A razão pela qual o produto é fechado em $\mathfrak{A}(X, \mathfrak{H})$ é digna de maiores comentários: Fixada uma base ortonormal $\left\{\eta_{k}\right\}_{k \in \mathbb{N}}$ de $\mathfrak{H}$, computamos

$$
\langle\phi, A B(x) \psi\rangle_{\mathfrak{H}}=\left\langle A(x)^{*} \phi, B(x) \psi\right\rangle_{\mathfrak{H}}=\sum_{k=1}^{\infty}\left\langle A(x)^{*} \phi, \eta_{k}\right\rangle_{\mathfrak{H}}\left\langle\eta_{k}, B(x) \psi\right\rangle_{\mathfrak{H}}=\sum_{k=1}^{\infty}\left\langle\phi, A(x) \eta_{k}\right\rangle_{\mathfrak{H}}\left\langle\eta_{k}, B(x) \psi\right\rangle_{\mathfrak{H}}
$$

o que nos permite aplicar as hipóteses, agora que $A(x)$ e $B(x)$ estão desacoplados. Como antes, se levamos em conta apenas as funções de $\Sigma(\Omega)$ que são constantes, $\mathfrak{A}(X, \mathfrak{H})$ torna-se uma $\mathbb{C}$-álgebra involutiva associativa unital, onde a aplicação que encaminha todo elemento de $X$ no operador nulo (respec. identidade) desempenha o papel de neutro aditivo (respec. multiplicativo).

Segue um compilado de algumas propriedades úteis acerca da ultima definição:

- Se $\left(C_{n}\right)_{n \in \mathbb{N}}$ é uma sequência em $\mathfrak{A}(X, \mathfrak{H})$ tal que $C_{n}(x) \stackrel{p}{\longrightarrow} C(x)$ para todo $x \in X$, então

$$
C_{n}(\cdot) \psi \in \mathcal{W}(X, \mathfrak{H}) \quad \forall \psi \in \mathfrak{H} \Longrightarrow C(\cdot) \psi \in \mathcal{W}(X, \mathfrak{H}) \quad \forall \psi \in \mathfrak{H} \Longrightarrow C \in \mathfrak{A}(X, \mathfrak{H})
$$

já que pela definição de convergência pontual, são equivalentes

$$
\begin{aligned}
C(x) \stackrel{p \cdot}{\longrightarrow} C(x) \quad \forall x \in X \Longleftrightarrow\|\cdot\|_{\mathfrak{H}}-\lim _{n \rightarrow \infty} C_{n}(x) \psi=C(x) \psi \quad \forall \psi \in \mathfrak{H}, x \in X & \Longleftrightarrow \\
& \Longleftrightarrow C_{n}(\cdot) \psi \stackrel{p \cdot}{\longrightarrow} C(\cdot) \psi \quad \forall \psi \in \mathfrak{H}
\end{aligned}
$$

invertendo-se a ordem dos quantificadores.

- $A \in \mathfrak{A}(X, \mathfrak{H})$ sempre faz de $X \ni x \longmapsto\|A(x)\|_{\text {op }} \in \mathbb{R}$ uma aplicação $\Omega$-mensurável, pois dado $\left\{\beta_{j}\right\}_{j \in \mathbb{N}}$ denso em $\mathbb{S}^{1}(\mathfrak{H})^{2}$, tem-se

$$
\|A(x)\|_{\text {op }}=\sup _{\psi, \phi \in \mathbb{S}^{1}(\mathfrak{H})}\left|\langle\phi, A(x) \psi\rangle_{\mathfrak{H}}\right| \stackrel{3}{=} \sup _{i, j \in \mathbb{N}}\left|\left\langle\beta_{j}, A(x) \beta_{i}\right\rangle_{\mathfrak{H}}\right|
$$

o que exprime-a como supremo de uma sequência de aplicações $\Omega$-mensuráveis.

Em função deste ultimo fato, cabe definir o conjunto

$$
\mathcal{L}_{\mathfrak{H}}^{\infty}(X, \Omega, \mu) \doteq\left\{A \in \mathfrak{A}(X, \mathfrak{H}): \operatorname{essip}_{x \in X}\|A(x)\|_{\text {op }}<+\infty\right\}
$$

que trivialmente forma uma $\mathbb{C}$-subálgebra involutiva associativa e com unidade de $\mathfrak{A}(X, \mathfrak{H})$, uma vez assimilado as propriedades e resultados básicos dos espaços $\mathrm{L}^{\infty}$ convencionais. Pelo mesmo motivo,

$$
r(A) \doteq \operatorname{ess~sup}_{x \in X}\|A(x)\|_{\text {op }}, \quad A \in \mathfrak{A}(X, \mathfrak{H})
$$

define uma seminorma em $\mathcal{L}_{\mathfrak{H}}^{\infty}(X)$. Na realidade, o subespaço $\mathcal{Q} \doteq r^{-1}(0)$ é um ideal autoadjunto de $\mathcal{L}_{\mathfrak{H}}^{\infty}(X)$ por que $r$ é submultiplicativo e a norma de operador não diferencia um operador de seu adjunto. Isso viabiliza o quociente

\footnotetext{
${ }^{2}$ No âmbito dos espaços métricos, a separabilidade é uma propriedade topológica hereditária, e logo herdada por cada subconjunto de $\mathfrak{H}$.

${ }^{3}$ Os supremos coincidem pois o segundo conjunto está contido no primeiro, e todo elemento do primeiro pode ser arbitrariamente aproximado por elementos do segundo, devido a continuidade das aplicações envolvidas.
} 


$$
\mathrm{L}_{\mathfrak{H}}^{\infty}(X, \Omega, \mu) \doteq \frac{\mathcal{L}_{\mathfrak{H}}^{\infty}(X, \Omega, \mu)}{\{A: r(A)=0\}}
$$

que por sua vez forma uma $\mathbb{C}$-álgebra normada involutiva associativa e com unidade, se munido adequadamente das operações de classe de equivalência

- $(A+\mathcal{Q})+(B+\mathcal{Q})=(A+B)+\mathcal{Q}$

- $\alpha(A+\mathcal{Q})=(\alpha A)+\mathcal{Q}$

(Multiplicação por Escalar)

- $(A+\mathcal{Q}) \cdot(B+\mathcal{Q})=A B+\mathcal{Q}$

(Produto)

- $(A+\mathcal{Q})^{*}=A^{*}+\mathcal{Q}$

(Involução)

- $\|A+\mathcal{Q}\|_{\infty, \mathfrak{H}} \doteq r(A)$

(Norma)

conforme é previsto pela teoria geral do quociente de álgebras por ideais.

Teorema 30. Seja $\mathfrak{H}$ um espaço de Hilbert separável e $(X, \Omega, \mu)$ um espaço de medida. Então, $\mathrm{L}_{\mathfrak{H}}^{\infty}(X, \Omega, \mu)$ forma uma álgebra $\mathrm{C}^{*}$.

Demonstração. Seja, pois, $\left(A_{n}+\mathcal{Q}\right)_{n \in \mathbb{N}}$ uma sequência de Cauchy em $\mathrm{L}_{\mathfrak{H}}^{\infty}(X)$. Isso significa que para um dado $\varepsilon>0$, existe $n_{0} \in \mathbb{N}$ de modo que

$$
\begin{aligned}
\left\|A_{m}(x)-A_{n}(x)\right\|_{\text {op }} \leq r\left(A_{m}-A_{n}\right)=\left\|\left(A_{m}-A_{n}\right)+\mathcal{Q}\right\|_{\infty, \mathfrak{H}} & \\
& =\left\|\left(A_{m}+\mathcal{Q}\right)-\left(A_{n}+\mathcal{Q}\right)\right\|_{\infty, \mathfrak{H}}<\varepsilon \quad \forall m, n>n_{0} \quad \mu \text {-q.t.p. }
\end{aligned}
$$

ou seja, $\left(A_{n}(x)\right)_{n \in \mathbb{N}}$ é de Cauchy em $\mu$-quase toda parte, e portanto convergente, em virtude da completude de $\mathcal{B}(\mathfrak{H})$. Denotando com $E$ o conjunto (de medida total) formado pelos pontos $x$ de $X$ para o qual $\left(A_{n}(x)\right)_{n \in \mathbb{N}}$ converge, ponha

$$
A(x) \doteq\|\cdot\|_{\mathrm{op}}-\lim _{n \rightarrow \infty} \chi_{E}(x) A_{n}(x)
$$

o que implica $\chi_{E}(x) A_{n}(x) \stackrel{p .}{\longrightarrow} A(x)$ e assegura $A \in \mathfrak{A}(X, \mathfrak{H})$. Dado um novo $\theta>0$, seja $m_{0}$ o respectivo inteiro positivo fornecido pelo critério de Cauchy como antes. Pondo

$$
G \doteq\left\{x \in X:\left\|A_{n}(x)-A_{m}(x)\right\|_{\text {op }}<\theta \quad \forall m, n>m_{0}\right\}
$$

veja que para todo $x \in G$ e $n>m_{0}$ que fixamos, a sequência numérica $\left(\left\|A_{n}(x)-A_{m}(x)\right\|_{\text {op }}\right)_{m \in \mathbb{N}}$ fica menor que $\theta$ a partir de um certo índice (a saber $m_{0}$ ), e com isso seu limite (caso exista) também não deve exceder esse mesmo valor. Isto posto,

$$
\left\|A_{n}(x)-A(x)\right\|_{\text {op }}=\lim _{m \rightarrow \infty}\left\|A_{n}(x)-A_{m}(x)\right\|_{\text {op }} \leq \theta \quad \forall x \in E \cap G, n>m_{0}
$$

donde extraímos duas informações valiosas, sendo a primeira

$$
\|A(x)\|_{\text {op }} \leq\left\|A_{m_{0}+1}(x)-A(x)\right\|_{\text {op }}+\left\|A_{m_{0}+1}(x)\right\|_{\text {op }} \leq \theta+r\left(A_{m_{0}+1}\right)<+\infty \quad \forall x \in E \cap G
$$

ou seja, $A$ é essencialmente limitado ${ }^{4}$, e portanto membro de $\mathcal{L}_{\mathfrak{H}}^{\infty}(X)$. Para todo $n>m_{0}, \theta$ também se mostrou um majorante essencial de $\left\|A_{n}(x)-A(x)\right\|_{\text {op }}$, e por isso

\footnotetext{
${ }^{4} 0 \leq \mu(X \backslash(E \cap G))=\mu((X \backslash E) \cup(X \backslash G)) \leq \mu(X \backslash E)+\mu(X \backslash G)=0+0=0 \Longrightarrow \mu(X \backslash(E \cap G))=0$.
} 


$$
\left\|\left(A_{n}+\mathcal{Q}\right)-(A+\mathcal{Q})\right\|_{\infty, \mathfrak{H}}=\left\|\left(A_{n}-A\right)+\mathcal{Q}\right\|_{\infty, \mathfrak{H}}=r\left(A_{n}-A\right) \leq \theta \quad \forall n>m_{0}
$$

Pela arbitrariedade do $\theta>0$ fornecido no início, segue

$$
\|\cdot\|_{\infty, \mathfrak{H}}-\lim _{n \rightarrow \infty}\left(A_{n}+\mathcal{Q}\right)=A+\mathcal{Q}
$$

e portanto a completeza de $\mathrm{L}_{\mathfrak{H}}^{\infty}(X)$. Por fim, não é difícil ver que as operações atribuídas à $\mathrm{L}_{\mathfrak{H}}^{\infty}(X)$ colaboram para que a submultiplicatividade da norma $\|\cdot\|_{\infty, \mathfrak{H}}$ e a propriedade $\mathrm{C}^{*}$ reincidam em $r$.

Por meio das mesmas justificativas de outrora, deixamos de lado a notação de classes de equivalência $A+Q$.

\subsection{Integral Direta de um Espaço de Hilbert}

"O matemático está envolvido num jogo do qual ele mesmo escreve as regras, enquanto o físico joga com as regras fornecidas pela natureza."

Paul Dirac ${ }^{5}$

Se $\mathfrak{H}$ é um espaço de Hilbert separável e $(X, \Omega, \mu)$ um espaço de medida, verifica-se sem qualquer dificuldade que $\|\cdot\|_{2, \mathfrak{H}}$ realiza a identidade do trapézio porque $\|\cdot\|_{\mathfrak{H}}$ o faz. Aplicando o célebre teorema de Jordan ${ }^{6}-$ Von-Neumann ${ }^{7}$, deduzimos que $\|\cdot\|_{2, \mathfrak{H}}$ provém de um produto interno (que denotamos por $\left.\langle\cdot, \cdot\rangle_{2, \mathfrak{H}}\right)$, e $\mathbf{L}_{\mathfrak{H}}^{2}(X)$ por sua vez define um espaço de Hilbert. Por fim, a identidade de polarização se presta na obtenção de uma fórmula explícita para o produto interno

$$
\langle f, g\rangle_{2, \mathfrak{H}}=\int_{X}\langle f(x), g(x)\rangle_{\mathfrak{H}} \mathrm{d} \mu(x), \quad f, g \in \mathrm{L}_{\mathfrak{H}}^{2}(X)
$$

Exemplo 31. Seja $\mathfrak{H}$ um espaço de Hilbert separável e $X$ um conjunto enumerável arbitrário. Então,

$$
\mathrm{L}_{\mathfrak{H}}^{2}(X, \mathbb{P}(X), \mathfrak{c}) \simeq \bigoplus_{n=1}^{|X|} \mathfrak{H}
$$

valendo a igualdade caso $X=\{1, \ldots, n\}$, em que $n \in \mathbb{N} \cup\{\infty\}$.

Com efeito, a $\sigma$-álgebra considerada faz com que toda e qualquer função de $X$ em $\mathbb{C}$ seja mensurável, e portanto que toda função de $X$ em $\mathfrak{H}$ seja fracamente mensurável. Invocando um dos resultados que acomodamos no apêndice $\mathrm{A}$, segue

$\mathrm{L}_{\mathfrak{H}}^{2}(X, \mathbb{P}(X), \mathfrak{c})=\left\{f: X \longrightarrow \mathfrak{H} \mid \sum_{x \in X}\|f(x)\|_{\mathfrak{H}}^{2}<+\infty\right\} \simeq\left\{v: \mathbb{N} \longrightarrow \mathfrak{H} \mid \sum_{k=1}^{|X|}\left\|v_{k}\right\|_{\mathfrak{H}}^{2}<+\infty\right\}=\bigoplus_{n=1}^{|X|} \mathfrak{H}$

onde a equivalência de espaços de Hilbert $\simeq$ se justifica no seguinte: Dada uma enumeração $\sigma$ de $X$, o mapa $f \longmapsto f \circ \sigma$ é claramente linear, tem $v \longmapsto v \circ \sigma^{-1}$ como inversa e cumpre

\footnotetext{
${ }^{5}$ Paul Adrien Maurice Dirac, $\odot(08 / 08 / 1902)-\dagger(20 / 10 / 1984)$

${ }^{6}$ Ernst Pascual Jordan, $\varnothing(18 / 10 / 1902)-\dagger(31 / 07 / 1980)$

${ }^{7}$ John von Neumann, $\odot(28 / 12 / 1903)-\dagger(08 / 02 / 1957)$
} 


$$
\|f\|_{2, \mathfrak{H}}^{2}=\sum_{x \in X}\|f(x)\|_{\mathfrak{H}}^{2}=\sum_{n=1}^{|X|}\|f \circ \sigma(n)\|_{\mathfrak{H}}^{2}=\|f \circ \sigma\|_{\oplus_{1}^{\infty} \mathfrak{H}}^{2}
$$

seja qual for $f \in \mathrm{L}_{\mathfrak{H}}^{2}(X)$.

O exemplo anterior sugere que $L_{\mathfrak{H}}^{2}$ seja uma generalização autêntica da noção de soma direta de espaços de Hilbert que usualmente concebemos. Por respeito a tradição, pelo apelo estético e, principalmente, porque isto valoriza o conceito heurístico discutido, redefinimos:

Definição 32 (Integral Direta de um Espaço de Hilbert). Seja $\mathfrak{H}$ um espaço de Hilbert separável e $(X, \Omega, \mu)$ um espaço de medida. A integral direta de $\mathfrak{H}$ em $X$ relativo à medida $\mu$ (ou simplesmente espaço de $\mathfrak{H}$-fibras) é definido como

$$
\int_{X}^{\oplus} \mathfrak{H} \mathrm{d} \mu \equiv \mathrm{L}_{\mathfrak{H}}^{2}(X, \Omega, \mu)
$$

A teoria moderna de integral direta dá conta de generalizar inclusive somas do tipo $\bigoplus_{1}^{\infty} \mathfrak{H}_{n}$, onde não somamos necessariamente sobre o mesmo $\mathfrak{H}$. Isso dá vida a expressões do tipo

$$
\int_{X}^{\oplus} \mathfrak{H}_{x} \mathrm{~d} \mu(x)
$$

em cujo caso $\left\{\mathfrak{H}_{x}\right\}_{x \in X}$ é uma família de espaços de Hilbert. Quanto a nós, permanecemos com a definição acima, visto que todo esplendor e glória do caso geral é completamente inócuo aos nossos objetivos.

Se $f$ e $g$ são duas funções que compartilham de um mesmo domínio, $(f, g)$ serve tanto para denotar o par ordenado quanto para designar o mapa $t \longmapsto(f(t), g(t))$. Afim de evitar uma ambiguidade na proposição que segue, suspendemos temporariamente a interpretação de mapa.

Proposição 33. Se $\mathfrak{H}_{1}$ e $\mathfrak{H}_{2}$ são espaços de Hilbert separáveis e $(X, \Omega, \mu)$ um espaço de medida, então

$$
\left(\int_{X}^{\oplus} \mathfrak{H}_{1} \mathrm{~d} \mu\right) \oplus\left(\int_{X}^{\oplus} \mathfrak{H}_{2} \mathrm{~d} \mu\right) \simeq_{\mathrm{U}} \int_{X}^{\oplus} \mathfrak{H}_{1} \oplus \mathfrak{H}_{2} \mathrm{~d} \mu
$$

onde o operador unitário $\mathrm{U}$ é definido por

$$
\mathrm{U}(f, g)(x) \doteq(f(x), g(x)) \quad \mu \text {-q.t.p }
$$

sejam quais forem $f \in \mathrm{L}_{\mathfrak{H}_{1}}^{2}(X)$ e $g \in \mathrm{L}_{\mathfrak{H}_{2}}^{2}(X)$.

Demonstração. $\mathrm{U}(f, g) \in \mathcal{W}\left(X, \mathfrak{H}_{1} \oplus \mathfrak{H}_{2}\right)$ porque suas funções coordenadas $f \in \mathcal{W}\left(X, \mathfrak{H}_{1}\right)$ e $g \in$ $\mathcal{W}\left(X, \mathfrak{H}_{2}\right)$, além do que

$$
\begin{aligned}
\int_{X}\|\mathrm{U}(f, g)(x)\|_{\mathfrak{H}_{1} \oplus \mathfrak{H}_{2}}^{2} \mathrm{~d} \mu(x)=\int_{X}\left(\|f(x)\|_{\mathfrak{H}_{1}}^{2}+\|g(x)\|_{\mathfrak{H}_{2}}^{2}\right) \mathrm{d} \mu(x)= & \\
& =\int_{X}\|f(x)\|_{\mathfrak{H}_{1}}^{2} \mathrm{~d} \mu(x)+\int_{X}\|g(x)\| \|_{\mathfrak{H}_{2}}^{2} \mathrm{~d} \mu(x)<+\infty
\end{aligned}
$$


visto que cada uma das parcelas acima é (por hipótese) finita. Em suma

$$
\mathrm{U}(f, g) \in \int_{X}^{\oplus} \mathfrak{H}_{1} \oplus \mathfrak{H}_{2} \mathrm{~d} \mu
$$

e portanto a boa definição de U. O cálculo anterior também provou

$$
\|\mathrm{U}(f, g)\|_{2, \mathfrak{H}_{1} \oplus \mathfrak{H}_{2}}^{2}=\|f\|_{2, \mathfrak{H}_{1}}^{2}+\|g\|_{2, \mathfrak{H}_{2}}^{2}=\|(f, g)\|_{\mathfrak{H}_{1} \oplus \mathfrak{H}_{2}}^{2}
$$

de forma que $\mathrm{U}$ conserva normas. Por fim, se $h \in \mathrm{L}_{\mathfrak{H}_{1} \oplus \mathfrak{H}_{2}}^{2}(X)$ e $\pi_{i}$ denota a projeção de $\mathfrak{H}_{1} \oplus \mathfrak{H}_{2}$ em $\mathfrak{H}_{i}$, evidentemente

$$
h=\mathrm{U}\left(\pi_{1} \circ h, \pi_{2} \circ h\right)
$$

o que nos confere a sobrejetividade de U, ficando a cargo do leitor sua linearidade.

\subsection{Operadores Decomponíveis em Fibras}

"Em algum lugar, alguma coisa incrível está esperando para ser descoberta."

\section{Carl Sagan $^{8}$}

Os espaços $L_{\mathfrak{H}}^{\infty}$ estão intimamente ligados a uma classe muito especial de operadores agindo em $L_{\mathfrak{H}}^{2}$, os quais descrevemos no que segue.

Teorema 34. Se $\mathfrak{H}$ um espaço de Hilbert separável e $(X, \Omega, \mu)$ um espaço de medida semifinito, então

$$
\begin{aligned}
& \pi: \mathrm{L}_{\mathfrak{H}}^{\infty}(X, \Omega, \mu) \longrightarrow \mathcal{B}\left(\int_{X}^{\oplus} \mathfrak{H} \mathrm{d} \mu\right) \\
& A \quad \longmapsto \int_{X}^{\oplus} A(x) \mathrm{d} \mu(x): \int_{X}^{\oplus} \mathfrak{H} \mathrm{d} \mu \longrightarrow \int_{X}^{\oplus} \mathfrak{H} \mathrm{d} \mu \\
& \Psi \quad \longmapsto \pi(A) \Psi \quad: \quad \longrightarrow \quad \mathfrak{H} \\
& x \longmapsto A(x) \Psi(x)
\end{aligned}
$$

define um homomorfismo de álgebra involutiva normada e unital.

Demonstração. Fixe $A \in \mathrm{L}_{\mathfrak{H}}^{\infty}(X), \Psi \in \mathrm{L}_{\mathfrak{H}}^{2}(X),\left\{\eta_{k}\right\}_{k \in \mathbb{N}}$ uma base ortonormal de $\mathfrak{H}$ e ponha

$$
\varphi_{n}(x) \doteq \sum_{k=1}^{n}\left\langle\eta_{k}, \Psi(x)\right\rangle_{\mathfrak{H}} A(x) \eta_{k}
$$

Assim definida, cada $\varphi_{n}$ é fracamente mensurável por que $\mathcal{W}(X, \mathfrak{H})$ é um $\Sigma(\Omega)$-módulo, $\eta_{k}^{*} \circ \Psi \in \Sigma(\Omega)$ e $A(\cdot) \eta_{k} \in \mathcal{W}(X, \mathfrak{H})$ para todo $k \in \mathbb{N}$. Em virtude da segunda identidade de Parseval, podemos exprimir cada vetor $\Psi(x)$ de $\mathfrak{H}$ através do limite

${ }^{8}$ Carl Edward Sagan, D(09/11/1934) - †(20/12/1996) 


$$
\Psi(x)=\|\cdot\|_{\mathfrak{H}^{-}}-\lim _{n \rightarrow \infty} \sum_{k=1}^{n}\left\langle\eta_{k}, \Psi(x)\right\rangle_{\mathfrak{H}} \eta_{k}
$$

que junto da continuidade e linearidade do operador $A(x)$ acarreta em

$$
\pi(A) \Psi(x)=A(x) \Psi(x)=\|\cdot\|_{\mathfrak{H}}-\lim _{n \rightarrow \infty} A(x)\left(\sum_{k=1}^{n}\left\langle\eta_{k}, \Psi(x)\right\rangle_{\mathfrak{H}} \eta_{k}\right)=\|\cdot\|_{\mathfrak{H}}-\lim _{n \rightarrow \infty} \varphi_{n}(x)
$$

Como acabamos de escrever $\pi(A) \Psi$ como limite pontual de uma sequência de aplicações fracamente mensuráveis, consuma-se

$$
\pi(A) \Psi \in \mathcal{W}(X, \mathfrak{H})
$$

Por conseguinte e as custas da desigualdade de Bessel, computa-se

$$
\left\|\varphi_{n}(x)\right\|_{\mathfrak{H}}^{2} \leq\|A(x)\|_{\text {op }}^{2} \sum_{k=1}^{n}\left|\left\langle\eta_{k}, \Psi(x)\right\rangle_{\mathfrak{H}}\right|^{2} \leq\|A\|_{\infty, \mathfrak{H}}^{2}\|\Psi(x)\|_{\mathfrak{H}}^{2} \quad \mu \text {-q.t.p }
$$

o que significa

$$
\left\|\varphi_{n}(\cdot)\right\|_{\mathfrak{H}}^{2} \leq\|A\|_{\infty, \mathfrak{H}}^{2}\|\Psi(\cdot)\|_{\mathfrak{H}}^{2} \in \mathrm{L}^{1}(X) \quad \forall n \in \mathbb{N}
$$

e graças ao teorema da convergência dominada,

$$
\int_{X}\|\pi(A) \Psi(x)\|_{\mathfrak{H}}^{2} \mathrm{~d} \mu(x)=\lim _{n \rightarrow \infty} \int_{X}\left\|\varphi_{n}(x)\right\|_{\mathfrak{H}}^{2} \mathrm{~d} \mu(x) \leq\|A\|_{\infty, \mathfrak{H}}^{2}\|\Psi\|_{2, \mathfrak{H}}^{2}<+\infty
$$

O fruto de todo nosso esforço manifesta-se na pertinência $\pi(A) \Psi \in \mathrm{L}_{\mathfrak{H}}^{2}(X)$, e portanto na boa definição de $\pi(A)$, uma vez que $\Psi$ é arbitrário. Encarregamos o leitor de se certificar que $\pi(A)$ é linear, e por efeito da expressão acima,

$$
\|\pi(A)\|_{\text {op }} \leq\|A\|_{\infty, \mathfrak{H}}
$$

o que finalmente assegura a boa definição de $\pi$, visto que $A$ também é arbitrário. A tarefa mecânica de que $\pi$ qualifica um homomorfismo de álgebra involutiva também não oferece desafio algum. Por exemplo, para todo $\Psi$ e $\Phi$ em $L_{\mathfrak{H}}^{2}(X)$, tem-se

$$
\begin{aligned}
&\langle\pi(A) \Psi, \Phi\rangle_{2, \mathfrak{H}}=\int_{X}\langleA(x) \Psi(x), \Phi(x)\rangle_{\mathfrak{H}} \mathrm{d} \mu(x)=\int_{X}\left\langle\Psi(x), A(x)^{*} \Phi(x)\right\rangle_{\mathfrak{H}} \mathrm{d} \mu(x)= \\
& \quad=\int_{X}\left\langle\Psi(x), A^{*}(x) \Phi(x)\right\rangle_{\mathfrak{H}} \mathrm{d} \mu(x)=\int_{X}\left\langle\Psi(x), \pi\left(A^{*}\right) \Phi(x)\right\rangle_{\mathfrak{H}} \mathrm{d} \mu(x)=\left\langle\Psi, \pi\left(A^{*}\right) \Phi\right\rangle_{2, \mathfrak{H}}
\end{aligned}
$$

o que implica $\pi(A)^{*}=\pi\left(A^{*}\right)$, e portanto que $\pi$ preserva involuções. Ilustrado a simplicidade de um desses fatos, o resto fica por conta do leitor, e conosco a tarefa mais envolvente de que $\pi$ preserva normas. Com efeito, se $\left\{\beta_{k}\right\}_{k \in \mathbb{N}}$ é denso em $\mathbb{S}^{1}(\mathfrak{H})$, assuma por um momento que seja possível decompor uma dada $f \in \mathrm{L}^{1}(X)$ num produto de duas funções $g, h \in \mathrm{L}^{2}(X)$ que cumprem mutuamente a relação

$$
\|g\|_{2}^{2}=\|h\|_{2}^{2}=\|f\|_{1}
$$

Definindo $\xi_{i j} \in \mathrm{L}^{\infty}(X)$ por $\xi_{i j}(x) \doteq\left\langle\beta_{j}, A(x) \beta_{i}\right\rangle_{\mathfrak{H}}$, tem-se

$$
\xi_{i j}(x) f(x)=g(x) h(x)\left\langle\beta_{j}, A(x) \beta_{i}\right\rangle_{\mathfrak{H}}=\left\langle\overline{g(x)} \beta_{j}, A(x)\left(h(x) \beta_{i}\right)\right\rangle_{\mathfrak{H}} \quad \forall x \in X
$$


e por força de um resultado acomodado no apêndice A,

$$
\begin{aligned}
\left|\Sigma_{\xi_{i j}}(f)\right|=\left|\left\langle\overline{g(\cdot)} \beta_{j}, \pi(A)\left(h(\cdot) \beta_{i}\right)\right\rangle_{2, \mathfrak{H}}\right| \leq\left\|\overline{g(\cdot)} \beta_{j}\right\|_{2, \mathfrak{H}}|| h(\cdot) \beta_{i}\left\|_{2, \mathfrak{H}}\right\| \pi(A) \|_{\text {op }}= \\
=\left\|\beta_{j}\right\|\left\|_{\mathfrak{H}}\right\| \beta_{i}\|\|_{\mathfrak{H}}\|g\|_{2}\|h\|_{2}\|\pi(A)\|_{\text {op }}=\|f\|_{1}\|\pi(A)\| \|_{\text {op }}
\end{aligned}
$$

Em atenção a arbitrariedade da $f$ solicitada, obtemos

$$
\left|\left\langle\beta_{j}, A(x) \beta_{i}\right\rangle_{\mathfrak{H}}\right| \leq\left\|\xi_{i j}\right\|_{\infty}=\left\|\Sigma_{\xi_{i j}}\right\|_{\text {op }} \leq\|\pi(A)\|_{\text {op }} \quad \mu \text {-q.t.p. } \quad \forall i, j \in \mathbb{N}
$$

e que trocando a ordem dos quantificadores ocasiona ${ }^{9}$

$$
\left|\left\langle\beta_{j}, A(x) \beta_{i}\right\rangle_{\mathfrak{H}}\right| \leq\|\pi(A)\|_{\text {op }} \quad \forall i, j \in \mathbb{N} \quad \mu \text {-q.t.p. }
$$

ou seja,

$$
\|A(x)\|_{\text {op }}=\sup _{i, j \in \mathbb{N}}\left|\left\langle\beta_{j}, A(x) \beta_{i}\right\rangle_{\mathfrak{H}}\right| \leq\|\pi(A)\|_{\text {op }} \quad \mu \text {-q.t.p. }
$$

e finalmente $\|A\|_{\infty, \mathfrak{H}} \leq\|\pi(A)\|_{\text {op. }}$ Como ultima consideração, a decomposição empregada em $f$ efetua-se com $g=|f|^{\frac{1}{2}}, h=f$ em $f^{-1}(0)$ e $h=f / g$ no complementar de $f^{-1}(0)$.

Corolário 35. Seja $\mathfrak{H}$ um espaço de Hilbert separável, $(X, \Omega, \mu)$ um espaço de medida semifinito e $B \in \mathrm{L}_{\mathfrak{H}}^{\infty}(X)$. Então,

$$
\int_{X}^{\oplus} B(x) \mathrm{d} \mu(x)=0 \Longleftrightarrow B(x)=0 \quad \mu \text {-q.t.p }
$$

Demonstração. Evidente, pois como $\pi$ é isométrica,

$$
\int_{X}^{\oplus} B(x) \mathrm{d} \mu(x)=0 \Longleftrightarrow\left\|\int_{X}^{\oplus} B(x) \mathrm{d} \mu(x)\right\|_{\mathrm{op}}=0 \Longleftrightarrow\|B\|_{\infty, \mathfrak{H}}=0 \Longleftrightarrow B(x)=0 \quad \mu \text {-q.t.p }
$$

Definição 36 (Operador Decomponível). Seja $\mathfrak{H}$ um espaço de Hilbert separável e $(X, \Omega, \mu)$ um espaço de medida semifinito. Um operador $T \in \mathcal{B}\left(\mathrm{L}_{\mathfrak{H}}^{2}(X)\right.$ ) diz-se decomponível (em fibras pela integral direta de $\mathfrak{H})$ quando existe $A \in \mathrm{L}_{\mathfrak{H}}^{\infty}(X)$ de modo que

$$
T=\int_{X}^{\oplus} A(x) \mathrm{d} \mu(x)
$$

Neste caso, denominamos os operadores $A(x)$ como fibras de $T$.

Reiteramos que as propriedades de homomorfismo de $\pi$ se convertem nas seguintes manipulações algébricas com integral direta de operadores:

- $\int_{X}^{\oplus} A(x)+B(x) \mathrm{d} \mu(x)=\int_{X}^{\oplus} A(x) \mathrm{d} \mu(x)+\int_{X}^{\oplus} B(x) \mathrm{d} \mu(x)$

\footnotetext{
${ }^{9}$ Lembre-se de que reunião enumerável de conjuntos de medida nula ainda possui medida nula.
} 


$$
\begin{aligned}
& \text { - } \int_{X}^{\oplus} A(x) B(x) \mathrm{d} \mu(x)=\left(\int_{X}^{\oplus} A(x) \mathrm{d} \mu(x)\right)\left(\int_{X}^{\oplus} B(x) \mathrm{d} \mu(x)\right) \\
& \text { - } \int_{X}^{\oplus} A(x)^{*} \mathrm{~d} \mu(x)=\left(\int_{X}^{\oplus} A(x) \mathrm{d} \mu(x)\right)^{*} \\
& \text { - } \int_{X}^{\oplus} \alpha A(x) \mathrm{d} \mu(x)=\alpha\left(\int_{X}^{\oplus} A(x) \mathrm{d} \mu(x)\right) \\
& \text { - }\left\|\int_{X}^{\oplus} A(x) \mathrm{d} \mu(x)\right\|_{\text {op }}=\operatorname{ess~sup}_{x \in X}\|A(x)\|_{\text {op }}
\end{aligned}
$$

Neste contexto, os teoremas de convergência de integrais abstratas se manifestam da seguinte forma:

Proposição 37 (Teorema da Convergência Pontual). Seja $\mathfrak{H}$ um espaço de Hilbert separável, $(X, \Omega, \mu)$ um espaço de medida semifinito e $\left(A_{n}\right)_{n \in \mathbb{N}}$ uma sequência de funçôes em $\mathbf{L}_{\mathfrak{H}}^{\infty}(X)$ uniformemente limitada. Se $A_{n}(x) \stackrel{p .}{\longrightarrow} A(x)$ para todo $x \in X$, então $A \in \mathrm{L}_{\mathfrak{H}}^{\infty}(X)$ e

$$
\mathrm{s}-\lim _{n \rightarrow \infty} \int_{X}^{\oplus} A_{n}(x) \mathrm{d} \mu(x)=\int_{X}^{\oplus} A(x) \mathrm{d} \mu(x)
$$

Demonstração. Pelo o que foi discutido duas seções atrás, $A \in \mathfrak{A}(X, \mathfrak{H})$. Ademais, uma constante $M>0$ que majora a norma de operador de todos os $A_{n}(x)$ também cumpre

$$
\left\|A_{n}(x) \psi\right\|_{\mathfrak{H}} \leq M\|\psi\|_{\mathfrak{H}} \quad \forall n \in \mathbb{N}, \psi \in \mathfrak{H}, x \in X
$$

e daí

$$
\|A(x) \psi\|_{\mathfrak{H}}=\lim _{n \rightarrow \infty}\left\|A_{n}(x) \psi\right\|_{\mathfrak{H}} \leq M\|\psi\|_{\mathfrak{H}} \quad \forall \psi \in \mathfrak{H}, x \in X
$$

ou seja,

$$
\|A(x)\|_{\text {op }} \leq M \quad \forall x \in X
$$

de modo que $A \in \mathrm{L}_{\mathfrak{H}}^{\infty}(X)$. Finalmente, seja qual for $\Psi \in \mathrm{L}_{\mathfrak{H}}^{2}(X)$, estima-se

$$
\begin{aligned}
\left\|A_{n}(x) \Psi(x)-A(x) \Psi(x)\right\|_{\mathfrak{H}} \leq \| A_{n}(x)- & A(x)\|\|_{\text {op }}\|\Psi(x)\|_{\mathfrak{H}} \leq \\
& \leq\left(\left\|A_{n}(x)\right\|_{\text {op }}+\|A(x)\| \|_{\text {op }}\right)\|\Psi(x)\|_{\mathfrak{H}} \leq 2 M\|\Psi(x)\|_{\mathfrak{H}} \quad \forall x \in X
\end{aligned}
$$

o que acarreta

$$
\left\|\pi\left(A_{n}\right) \Psi(\cdot)-\pi(A) \Psi(\cdot)\right\|_{\mathfrak{H}}^{2} \leq(2 M)^{2}\|\Psi(\cdot)\|_{\mathfrak{H}}^{2} \in \mathrm{L}^{1}(X)
$$

e o teorema da convergência dominada se aplica, resultando

$$
\lim _{n \rightarrow \infty}\left\|\pi\left(A_{n}\right) \Psi-\pi(A) \Psi\right\|_{2, \mathfrak{H}}^{2}=\lim _{n \rightarrow \infty} \int_{X}\left\|A_{n}(x) \Psi(x)-A(x) \Psi(x)\right\|_{\mathfrak{H}}^{2} \mathrm{~d} \mu(x)=0
$$


Exemplo 38. Se $(X, \Omega, \mu)$ é um espaço de medida semifinito e $\mathrm{M}_{f}$ o operador de multiplicação por $f \in \mathrm{L}^{\infty}(X)$ agindo em $\mathrm{L}^{2}(X)$, então

$$
\mathrm{M}_{f}=\int_{X}^{\oplus} f(x) \mathrm{d} \mu(x)
$$

Bem entendido, $f(x)$ denota a homotetia ${ }^{10}$ que multiplica todo número complexo por $f(x)$. Em particular,

$$
\left\|\mathrm{M}_{f}\right\|_{\mathrm{op}}=\|f\|_{\infty}
$$

Tendo em vista $\mathcal{W}(X, \mathbb{C})=\Sigma(\Omega)$ e $|f(x)|=\|f(x)\|_{\text {op }}$ para todo $x \in X$, o resto é questão meramente tautológica $^{11}$.

Exemplo 39. Seja $(X, \mathcal{T})$ um espaço topológico, $\mu$ uma medida semifinita definida nos borelianos de $X$ e $f: X \longrightarrow \mathrm{L}^{\infty}(X)$ contínua e $\mu$-essencialmente limitada. Logo,

$$
\mathcal{L}_{f} \doteq \int_{X}^{\oplus} \mathrm{M}_{f(x)} \mathrm{d} \mu(x)
$$

define uma espécie de operador de multiplicação na integral direta de $\mathrm{L}^{2}(X)$ pois age como $h \longmapsto f h$, e além disso

$$
\left\|\mathcal{L}_{f}\right\|_{\text {op }}=\operatorname{ess}_{x \in X}\|f(x)\|_{\infty}
$$

$\mathrm{M}_{f(\cdot)}$ reflete as hipóteses impostas sobre $f$ porque é composição desta com o mapa isométrico

$$
\mathrm{L}^{\infty}(X) \in g \longmapsto \mathrm{M}_{g} \in \mathcal{B}\left(\int_{X}^{\oplus} \mathbb{C} \mathrm{d} \mu\right)
$$

Logo $\mathcal{L}_{f}$ define um elemento legítimo do $\mathrm{L}^{\infty}$ de $\mathrm{L}^{2}(X)$, cumprindo

$$
\mathcal{L}_{f} h[y]=\mathrm{M}_{f[y]} h[y]=f[y] h[y]=f h[y] \quad \mu \text {-q.t.p }
$$

conforme sugere o enunciado.

O próximo resultado mostra como a integral direta de operadores reage ao cálculo funcional boreliano, e como podemos extrair propriedades espectrais a partir disto.

\footnotetext{
${ }^{10}$ Um operador linear diz-se uma homotetia quando for múltiplo do operador identidade. Todo operador linear agindo em um espaço vetorial de dimensão 1 é necessariamente uma homotetia.

${ }^{11}$ Proposição analítica que permanece sempre verdadeira, uma vez que o atributo é uma repetição do sujeito. Exemplo: $O$ sal é salgado.
} 
Teorema 40. Seja $(X, \Omega, \mu)$ um espaço de medida semifinito, $\mathfrak{H}$ um espaço de Hilbert separável e

$$
T=\int_{X}^{\oplus} A(x) \mathrm{d} \mu(x)
$$

tal que suas fibras sejam todas autoadjuntas. São verdadeiras as seguintes sentenças:

a) Para toda $f \in \mathfrak{B}_{\mathrm{c}}(\mathbb{R}), f(T)$ é igualmente decomponível, e tem $f(A(x))$ como fibras. Simbolicamente,

$$
f\left(\int_{X}^{\oplus} A(x) \mathrm{d} \mu(x)\right)=\int_{X}^{\oplus} f(A(x)) \mathrm{d} \mu(x)
$$

b) $\lambda$ é autovalor de $T$ se, e somente se,

$$
\mu(\{x \in X: \lambda e ́ \text { autovalor de } A(x)\})>0
$$

c) $\lambda \in \sigma(T)$ se, e somente se,

$$
\forall \varepsilon>0, \quad \mu(\{x \in X: \sigma(A(x)) \cap(\lambda-\varepsilon, \lambda+\varepsilon) \neq \varnothing\})>0
$$

Demonstração. $T$ é claramente autoadjunto pois $A \in \mathrm{L}_{\mathfrak{H}}^{\infty}(X)$ é autoadjunto e $\pi$ definida no (T.34) preserva involuções. Pois bem,

$$
\mathcal{M} \doteq\left\{f \in \mathfrak{B}_{\mathrm{c}}(\mathbb{R}): f(A(\cdot)) \in \mathfrak{A}(X, \mathfrak{H})\right\}
$$

contém $\mathbb{C}[t \in \mathbb{R}]$ por razões óbvias, e visto que toda $f \in \mathrm{C}(\mathbb{R})$ pode ser aproximada na topologia da convergência compacta por uma sequência de polinômios $\left(p_{n}\right)_{n \in \mathbb{N}}$, a continuidade do cálculo funcional de cada uma das fibras de $T$ se aplica, de forma que

$$
\|\cdot\|_{\mathrm{op}}-\lim _{n \rightarrow \infty} p_{n}(A(x))=f(A(x)) \Longrightarrow p_{n}(A(x)) \stackrel{p \cdot}{\longrightarrow} f(A(x)) \Longrightarrow f(A(\cdot)) \in \mathfrak{A}(X, \mathfrak{H}) \Longrightarrow f \in \mathcal{M}
$$

Agora, se $\left(f_{n}\right)_{n \in \mathbb{N}}$ é uma sequência em $\mathcal{M}$ uniformemente limitada nos compactos e tal que $f_{n} \stackrel{p \text {. }}{\longrightarrow} f$, uma outra propriedade do cálculo aplicado a cada fibra diz que

$$
f_{n}(A(x)) \stackrel{p \cdot}{\longrightarrow} f(A(x))
$$

e de novo $f \in \mathcal{M}$. Pelo $(\mathrm{T} .5)$, só pode ser $\mathcal{M}=\mathfrak{B}_{\mathrm{c}}(\mathbb{R})$. Sendo $K \doteq\left[-\|T\|_{\mathrm{op}},\|T\|_{\mathrm{op}}\right]$, em verdade

$$
\|f(A(x))\|_{\text {op }} \leq \sup _{\sigma(A(x))}|f| \leq \sup _{K}|f|
$$

para toda $f \in \mathfrak{B}_{\mathrm{c}}(\mathbb{R})$ e $\mu$-quase todo $x \in X$, de modo que

$$
\mathcal{L}[f] \doteq \int_{X}^{\oplus} f(A(x)) \mathrm{d} \mu(x), \quad f \in \mathfrak{B}_{\mathrm{c}}(\mathbb{R})
$$

encontra-se bem definida. Para concluir a prova o item a), basta então mostrar que $\mathcal{L}$ cumpre todas as cláusulas que definem univocamente o cálculo funcional boreliano de T. Com efeito,

$$
\|\mathcal{L}[f]\|_{\text {op }}=\operatorname{essips}_{x \in X}\|f(A(x))\|_{\text {op }} \leq \sup _{K}|f|
$$


para toda $f \in \mathfrak{B}_{\mathrm{c}}(\mathbb{R})$,

$$
\mathcal{L}[\mathrm{id}]=\int_{X}^{\oplus} A(x) \mathrm{d} \mu(x)=T
$$

e se $\left(f_{n}\right)_{n \in \mathbb{N}}$ é uma sequência em $\mathcal{M}$ uniformemente limitada nos compactos e tal que $f_{n} \stackrel{p .}{\longrightarrow} f$, em particular

$$
|| f_{n}(A(x)) \|_{\text {op }} \leq \sup _{K}\left|f_{n}\right| \leq \sup _{n, K}\left|f_{n}\right|<+\infty
$$

mas também já vimos que $f_{n}(A(x)) \stackrel{p .}{\longrightarrow} f(A(x))$. Deferidas as premissas do (T.37), segue

$$
\mathcal{L}\left[f_{n}\right] \stackrel{p .}{\longrightarrow} \mathcal{L}[f]
$$

Em atenção as manipulações algébricas com integrais diretas, $\mathcal{L}$ também é um homomorfismo de álgebra involutiva com unidade, e acabamos o primeiro item. Na sequência, segue do (C.35) e da caracterização de um autovalor advinda do (P.11) que

$$
\begin{aligned}
\lambda \text { é autovalor de } T & \Longleftrightarrow \int_{X}^{\oplus} \mathrm{P}_{\{\lambda\}}(A(x)) \mathrm{d} \mu(x)=\mathrm{P}_{\{\lambda\}}\left(\int_{X}^{\oplus} A(x) \mathrm{d} \mu(x)\right) \neq 0 \\
& \Longleftrightarrow \mu\left(\left\{x \in X: \mathrm{P}_{\{\lambda\}}(A(x)) \neq 0\right\}\right)>0 \\
& \Longleftrightarrow \mu(\{x \in X: \lambda \text { é autovalor de } A(x)\})>0
\end{aligned}
$$

Por fim, resulta do (C.35)

$$
\begin{aligned}
\lambda \in \sigma(T) & \Longleftrightarrow \forall \varepsilon>0, \quad \int_{X}^{\oplus} \mathrm{P}_{(\lambda-\varepsilon, \lambda+\varepsilon)}(A(x)) \mathrm{d} \mu(x)=\mathrm{P}_{(\lambda-\varepsilon, \lambda+\varepsilon)}\left(\int_{X}^{\oplus} A(x) \mathrm{d} \mu(x)\right) \neq 0 \\
& \Longleftrightarrow \forall \varepsilon>0, \quad \mu\left(\left\{x \in X: \mathrm{P}_{(\lambda-\varepsilon, \lambda+\varepsilon)}(A(x)) \neq 0\right\}\right)>0 \\
& \Longleftrightarrow \forall \varepsilon>0, \quad \mu(\{x \in X: \sigma(A(x)) \cap(\lambda-\varepsilon, \lambda+\varepsilon) \neq \varnothing\})>0
\end{aligned}
$$

com um comentário adicional na ultima passagem: Se o intervalo $(\lambda-\varepsilon, \lambda+\varepsilon)$ não possui pontos espectrais de $A(x)$, a (P.11) indica que $\mathrm{P}_{(\lambda-\varepsilon, \lambda+\varepsilon)}(A(x))$ é nulo. Reciprocamente, se este projetor é nulo, mas o intervalo $(\lambda-\varepsilon, \lambda+\varepsilon)$ acomoda algum ponto espectral $\mu$ de $A(x)$, então dado $\delta>0$ de modo que $(\mu-\delta, \mu+\delta)$ esteja contido em $(\lambda-\varepsilon, \lambda+\varepsilon)$, teríamos pela (P.11)

$$
0 \leq \mathrm{P}_{(\mu-\delta, \mu+\delta)}(A(x)) \leq \mathrm{P}_{(\lambda-\varepsilon, \lambda+\varepsilon)}(A(x))=0
$$

mas em compensação viola-se o que foi firmado no (T.13).

Neste ponto, esperamos que a notação heurística de integral direta esteja justificada. Para concluir, mostraremos a força das integrais diretas de von Neumann provando alguns resultados conhecidos de forma rápida e elegante, como é o caso do:

Corolário 41. Seja $(X, \Omega, \mu)$ um espaço de medida semifinito e $f \in \mathrm{L}^{\infty}(X)$. São verdadeiras as seguintes alegações:

a) Para todo $h \in \mathfrak{B}_{\mathrm{c}}(\mathbb{R})$, tem-se $h\left(\mathrm{M}_{f}\right)=\mathrm{M}_{h \circ f}$.

b) $\sigma\left(\mathrm{M}_{f}\right)=\operatorname{im} \operatorname{ess}(f)$

Demonstração. Evidente, pois em reverência ao (Ex.38), tem-se 


$$
h\left(\mathrm{M}_{f}\right)=h\left(\int_{X}^{\oplus} f(x) \mathrm{d} \mu(x)\right)=\int_{X}^{\oplus} h(f(x)) \mathrm{d} \mu(x)=\mathrm{M}_{h \circ f}
$$

bem como

$$
\begin{aligned}
\lambda \in \sigma\left(\mathrm{M}_{f}\right) & \Longleftrightarrow \forall \varepsilon>0, \mu(\{x \in X: \sigma(f(x)) \cap(\lambda-\varepsilon, \lambda+\varepsilon) \neq \varnothing\})>0 \\
& \Longleftrightarrow \forall \varepsilon>0, \mu(\{x \in X:\{f(x)\} \cap(\lambda-\varepsilon, \lambda+\varepsilon) \neq \varnothing\})>0 \\
& \Longleftrightarrow \forall \varepsilon>0, \mu(\{x \in X:|\lambda-f(x)|<\varepsilon\})>0 \\
& \Longleftrightarrow \lambda \in \operatorname{essim}(f)
\end{aligned}
$$

e o corolário está provado.

Exemplo 42. Seja $X \in \mathscr{L}\left(\mathbb{R}^{n}\right)$ e $f \in \mathrm{L}^{\infty}(X)$ tal que a pré-imagem de todo conjunto com medida de Lebesgue nula tenha medida nula ${ }^{12}$. Então,

$$
\mathrm{L}^{2}(X)_{\mathrm{ac}}\left(\mathrm{M}_{f}\right)=\mathrm{L}^{2}(X)
$$

De fato, pois independente de qual seja o boreliano $G$ de $\mathbb{R}$,

$$
\mathrm{P}_{G}\left(\mathrm{M}_{f}\right)=\mathrm{M}_{\chi_{G} \circ f}=\mathrm{M}_{\chi_{f^{-1}(G)}}
$$

e portanto

$$
\Omega_{h}(G)=\int_{\mathbb{R}} \chi_{G} \mathrm{~d} \Omega_{h}=\left\langle h, \mathrm{P}_{G}\left(\mathrm{M}_{f}\right) h\right\rangle_{2}=\left\langle h, \chi_{f^{-1}(G)} h\right\rangle_{2}=\int_{f^{-1}(G)}|h(x)|^{2} \mathfrak{m}\left(\mathrm{d}^{n} x\right)
$$

para todo for $h \in \mathrm{L}^{2}(X)$.

\footnotetext{
${ }^{12}$ Isso ocorre, por exemplo, quando $X$ é um aberto de $\mathbb{R}^{n}$ e $f$ real analítica não constante.
} 


\section{Capítulo 3}

\section{Mecânica Quântica}

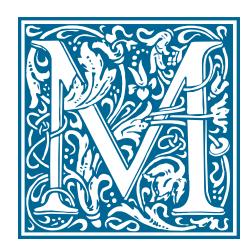

ECÂNICA QUÂNTICA, ou ainda, física quântica, é como denominamos a área da física que se encarrega de estudar e descrever de modo adequado as leis naturais que regem o universo em escalas atômicas. Ela surgiu da impossibilidade de se atribuir uma explicação satisfatória à certos fenômenos através da então física clássica, ou seja, a partir das leis que regem o universo em escalas macroscópicas. O termo "quântico" refere-se a uma unidade discreta que a teoria atribui a certas grandezas físicas observáveis. Apesar das desavenças filosóficas que a mecânica quântica promove, ela é o sustentáculo de todo o mecanismo da atual era digital e a única das teorias físicas a possuir um sistema axiomático coeso.

Noções Gerais e Teoremas: Produto Tensorial Simétrico e Alternado, Determinante e Permanente de Slater, Espaço Bosônico e Fermiônico de Muitos Corpos, Vácuo Quântico, Operador de Criação e Aniquilação Fermiônico, Entrelaçamento (Emaranhamento) Quântico, Princípio de Exclusão de Pauli, Operador de Criação e Aniquilação Bosônico e Fermiônico, Operador Número de Partículas Bosônicas e Fermiônicas, Grupo Unitário (à um Parâmetro Real) Fortemente Contínuo, Equações Diferenciais Lineares Autônomas em Espaços de Hilbert, Gerador Infinitesimal de um Grupo Unitário, Dinâmica Livre e Interagente, Estados Assintoticamente Livres num Futuro e Passado Distantes, Operador de Onda de Kato (Completo), Regra da Cadeira, Teorema de Pearson, Teorema de Kato-Rosenblum.

\subsection{Segunda Quantização}

"Deus não joga dados com o universo."

Albert Einstein

Nota Histórica. Foram muitos os físicos que se opuseram a formulação da mecânica quântica, por ser intrinsecamente probabilística. Tanto Einstein como o próprio Schrödinger tiveram dificuldades em aceitála, debatendo exaustivamente sobre o assunto. Numa dessas ocasiões, Einstein expressa seu desconforto sobre o assunto proferindo a bendita frase, talhada na literatura científica desde então.

A teoria geral determina que se $\mathfrak{H}$ é um espaço de Hilbert separável que descreve o espaço de estados de um sistema quântico composto por uma determinada partícula, então $\mathfrak{H}^{\otimes n}$ representa o espaço de estados do sistema composto por $n$ dessas partículas. Dependendo ainda do tipo de partícula que se lida (bósons ou férmions), há certas simetrias envolvendo o sistema físico em questão que sugerem uma atenção especial a certos subespaços de $\mathfrak{H}^{\otimes n}$, os quais descrevemos no que segue. 
Definição 43 (de Produto Tensorial Simétrico e Alternado). O produto tensorial alternado de uma $(k \geq 2)$-upla de vetores $u_{1}, \ldots, u_{k}$ em um espaço de Hilbert separável $\mathfrak{H}$ é definido por

$$
u_{1} \wedge \cdots \wedge u_{k} \doteq \frac{1}{\left|\mathcal{S}_{k}\right|} \sum_{\sigma \in \mathcal{S}_{k}} \operatorname{sgn}(\sigma) u_{\sigma(1)} \otimes \cdots \otimes u_{\sigma(k)}
$$

e o simétrico

$$
u_{1} \odot \cdots \odot u_{k} \doteq \frac{1}{\left|\mathcal{S}_{k}\right|} \sum_{\sigma \in \mathcal{S}_{k}} u_{\sigma(1)} \otimes \cdots \otimes u_{\sigma(k)}
$$

Eis alguns fatos gerais à respeito de um grupo $G$ e um conjunto arbitrário $X$ que estaremos invocando ao longo da presente seção:

- Para todo $h \in G$, o mapa $G \ni g \stackrel{\varrho_{h}}{\longmapsto} g h \in G$ define um automorfismo denominado translação $\grave{a}$ direita por $h$.

- $\mathcal{S}(X) \ni \sigma \longmapsto \operatorname{sgn}(\sigma) \in\{-1,1\}$ qualifica um homomorfismo de grupos.

Proposição 44. Seja $u_{1}, \ldots, u_{k}$ uma $(k \geq 2)$-upla de vetores em um espaço de Hilbert separável $\mathfrak{H}$. As seguintes afirmações são verdadeiras:

a) $u_{\varepsilon(1)} \wedge \cdots \wedge u_{\varepsilon(k)}=\operatorname{sgn}(\varepsilon) u_{1} \wedge \cdots \wedge u_{k} \quad \forall \varepsilon \in \mathcal{S}_{k}$

b) O produto tensorial alternado é multilinear nos seus parâmetros.

c) $u_{1} \wedge \cdots \wedge u_{k}$ se anula sempre que $\left\{u_{1}, \ldots, u_{k}\right\}$ for linearmente dependente.

d) Dada uma segunda $k$-upla $w_{1}, \ldots, w_{k} \in \mathfrak{H}$, tem-se

$$
\begin{aligned}
\left\langle u_{1} \wedge \cdots \wedge u_{k}, w_{1} \wedge \cdots \wedge w_{k}\right\rangle_{\mathfrak{H} \otimes k} & =\left\langle u_{1} \wedge \cdots \wedge u_{k}, w_{1} \otimes \cdots \otimes w_{k}\right\rangle_{\mathfrak{H} \otimes k} \\
& =\left\langle u_{1} \otimes \cdots \otimes u_{k}, w_{1} \wedge \cdots \wedge w_{k}\right\rangle_{\mathfrak{H} \otimes k} \\
& =(1 / k) ! \operatorname{det}\left(\left\langle u_{i}, w_{j}\right\rangle_{\mathfrak{H}}\right)_{i, j=1}^{k}
\end{aligned}
$$

Demonstração. Conforme nos adiantamos, $\varepsilon$ tem mesmo sinal que sua inversa por força da relação $\mathcal{S}_{k} \simeq_{\text {sgn }}\{-1,1\}$, e assim

$$
\begin{aligned}
u_{\varepsilon(1)} \wedge \cdots \wedge u_{\varepsilon(k)} & =\frac{1}{\left|\mathcal{S}_{k}\right|} \sum_{\sigma \in \mathcal{S}_{k}} \operatorname{sgn}(\sigma) u_{\sigma(\varepsilon(1))} \otimes \cdots \otimes u_{\sigma(\varepsilon(k))} \\
& =\operatorname{sgn}\left(\varepsilon^{-1}\right)\left(\frac{1}{\left|\mathcal{S}_{k}\right|} \sum_{\sigma \in \mathcal{S}_{k}} \operatorname{sgn}(\sigma \varepsilon) u_{\sigma \varepsilon(1)} \otimes \cdots \otimes u_{\sigma \varepsilon(k)}\right) \\
& =\operatorname{sgn}(\varepsilon) u_{1} \wedge \cdots \wedge u_{k}
\end{aligned}
$$

também por que $\varrho_{\varepsilon} \in \operatorname{Aut}\left(\mathcal{S}_{k}\right)$. Adiante, suponha que $u_{m}=u_{n}$ para algum $1 \leq m, m \leq k$ com $m \neq n$. Pondo $\tau=(m n) \in \mathcal{S}_{k}$, computamos 


$$
u_{1} \wedge \cdots \wedge u_{k}=u_{\tau(1)} \wedge \cdots \wedge u_{\tau(k)}=-u_{1} \wedge \cdots \wedge u_{k}
$$

o que implica $u_{1} \wedge \cdots \wedge u_{k}=0$, uma vez que a característica dos complexos é $\neq 2$. Provado que o produto tensorial alternado dos $u_{i}$ se anula sempre que houver alguma repetição dentre esses vetores, o caso geral resulta da multilinearidade de $\wedge$, e que por sua vez é fruto da multilinearidade de $\otimes$. Em sequência,

$$
\begin{aligned}
\left\langle u_{1} \wedge \cdots \wedge u_{k}, w_{1} \wedge \cdots \wedge w_{k}\right\rangle_{\mathfrak{H}}^{\otimes k} & =\frac{1}{\left|\mathcal{S}_{k}\right|^{2}} \sum_{\sigma \in \mathcal{S}_{k}} \operatorname{sgn}(\sigma)\left(\sum_{\varepsilon \in \mathcal{S}_{k}} \operatorname{sgn}(\varepsilon)\left\langle u_{\sigma(1)} \otimes \cdots \otimes u_{\sigma(k)}, w_{\varepsilon(1)} \otimes \cdots \otimes w_{\varepsilon(k)}\right\rangle_{\mathfrak{H} \otimes k}\right) \\
& =\frac{1}{\left|\mathcal{S}_{k}\right|^{2}} \sum_{\sigma \in \mathcal{S}_{k}} \operatorname{sgn}(\sigma)\left(\sum_{\varepsilon \in \mathcal{S}_{k}} \operatorname{sgn}(\varepsilon)\left\langle u_{\sigma(1)}, w_{\varepsilon(1)}\right\rangle_{\mathfrak{H}} \cdots\left\langle u_{\sigma(k)}, w_{\varepsilon(k)}\right\rangle_{\mathfrak{H}}\right) \\
& =\frac{1}{\left|\mathcal{S}_{k}\right|^{2}} \sum_{\sigma \in \mathcal{S}_{k}} \operatorname{sgn}(\sigma)\left(\sum_{\varepsilon \in \mathcal{S}_{k}} \operatorname{sgn}\left(\varepsilon \sigma^{-1} \sigma\right)\left\langle u_{\sigma(1)}, w_{\varepsilon \sigma^{-1}(\sigma(1))}\right\rangle_{\mathfrak{H}} \cdots\left\langle u_{\sigma(k)}, w_{\varepsilon \sigma^{-1}(\sigma(k))}\right\rangle_{\mathfrak{H}}\right) \\
& =\frac{1}{\left|\mathcal{S}_{k}\right|^{2}} \sum_{\sigma \in \mathcal{S}_{k}} \operatorname{sgn}(\sigma)^{2}\left(\sum_{\varepsilon \in \mathcal{S}_{k}} \operatorname{sgn}\left(\varepsilon \sigma^{-1}\right)\left\langle u_{1}, w_{\varepsilon \sigma^{-1}(1)}\right\rangle_{\mathfrak{H}} \cdots\left\langle u_{k}, w_{\varepsilon \sigma^{-1}(k)}\right\rangle_{\mathfrak{H}}\right) \\
& =\frac{1}{\left|\mathcal{S}_{k}\right|^{2}} \sum_{\sigma \in \mathcal{S}_{k}} \operatorname{det}\left(\left\langle u_{i}, w_{j}\right\rangle_{\mathfrak{H}}\right)_{i, j=1}^{k}=\frac{1}{\left|\mathcal{S}_{k}\right|} \operatorname{det}\left(\left\langle u_{i}, w_{j}\right\rangle_{\mathfrak{H}}\right)_{i, j=1}^{k}
\end{aligned}
$$

observando que na quarta passagem se aproveitamos da comutatividade em $\mathbb{C}$ para reordenar os produtos internos, e na quinta e ultima que $\varrho_{\sigma}^{-1}=\varrho_{\sigma^{-1}} \in \operatorname{Aut}\left(\mathcal{S}_{k}\right)$. Por fim

$$
\begin{aligned}
\left.\left\langle u_{1} \otimes \cdots \otimes u_{k}, w_{1} \wedge \cdots \wedge w_{k}\right)\right\rangle_{\mathfrak{H}^{\otimes k}} & =\frac{1}{\left|\mathcal{S}_{k}\right|} \sum_{\sigma \in \mathcal{S}_{k}} \operatorname{sgn}(\sigma)\left\langle u_{1} \otimes \cdots \otimes u_{k}, w_{\sigma(1)} \otimes \cdots \otimes w_{\sigma(k)}\right\rangle_{\mathfrak{H}^{\otimes k}} \\
& =\frac{1}{\left|\mathcal{S}_{k}\right|} \sum_{\sigma \in \mathcal{S}_{k}} \operatorname{sgn}(\sigma)\left\langle u_{1}, w_{\sigma(1)}\right\rangle_{\mathfrak{H}} \cdots\left\langle u_{k}, w_{\sigma(k)}\right\rangle_{\mathfrak{H}} \\
& =\frac{1}{\left|\mathcal{S}_{k}\right|} \operatorname{det}\left(\left\langle u_{i}, w_{j}\right\rangle_{\mathfrak{H}}\right)_{i, j=1}^{k}
\end{aligned}
$$

e analogamente

$$
\left\langle u_{1} \wedge \cdots \wedge u_{k}, w_{1} \otimes \cdots \otimes w_{k}\right\rangle_{\mathfrak{H} \otimes k}=\frac{1}{\left|\mathcal{S}_{k}\right|} \operatorname{det}\left(\left\langle u_{j}, w_{i}\right\rangle_{\mathfrak{H}}\right)_{i, j=1}^{k}=\frac{1}{\left|\mathcal{S}_{k}\right|} \operatorname{det}\left(\left\langle u_{i}, w_{j}\right\rangle_{\mathfrak{H}}\right)_{i, j=1}^{k}
$$

uma vez que toda matriz tem mesmo determinante que sua transposta.

Grande parte dos físicos e químicos reconhecem a ultima expressão do item d) como sendo o Determinante de Slater ${ }^{1}$ dos vetores $u_{1}, \ldots, u_{k}$ no ponto $\left(w_{1}, \ldots, w_{k}\right)$. Para darmos continuidade em nossa exposição, fixe $\Omega$ uma base ortonormal de $\mathfrak{H}$. Para cada $\sigma \in \mathcal{S}_{k}$, existe um único operador linear

$$
\mathrm{U}_{\sigma}:\left\langle\otimes^{k} \Omega\right\rangle \leq \mathfrak{H}^{\otimes k} \longrightarrow \mathfrak{H}^{\otimes k}
$$

satisfazendo

$$
\mathrm{U}_{\sigma}\left(e_{1} \otimes \cdots \otimes e_{k}\right)=e_{\sigma(1)} \otimes \cdots \otimes e_{\sigma(k)}
$$

\footnotetext{
${ }^{1}$ John Clarke Slater, $\odot(22 / 12 / 1900)-\dagger(25 / 07 / 1976)$
} 
para toda $k$-upla $e_{1}, \ldots, e_{k} \in \Omega$. Assim definido, $\mathrm{U}_{\sigma}$ é claramente limitado, e por consequência

$$
\Lambda_{k} \doteq \frac{1}{\left|\mathcal{S}_{k}\right|} \sum_{\sigma \in \mathcal{S}_{k}} \operatorname{sgn}(\sigma) \mathrm{U}_{\sigma}
$$

admite uma única extensão definida em todo $\mathfrak{H}^{\otimes k}$ que também seja linear e limitada, igualmente denotada por $\Lambda_{k}$. Quando uma $k$-upla $e_{1}, \ldots, e_{k}$ é tomada em $\Omega$, vale

$$
\Lambda_{k}\left(e_{1} \otimes \cdots \otimes e_{k}\right)=e_{1} \wedge \cdots \wedge e_{k}
$$

sendo que essa ação persiste num tensor puro genérico: Primeiro se verifica a validade da expressão para toda $k$-upla em $\langle\Omega\rangle$, e o caso geral segue por passagem ao limite. Daí resulta que a construção de $\Lambda_{k}$ não depende da escolha da base $\Omega$ que fixamos no início, dado que sua ação num tensor puro não faz menção aos vetores de $\Omega$. Por fim,

$$
\begin{aligned}
\Lambda_{k}\left(u_{1} \wedge \cdots \wedge u_{k}\right) & =\frac{1}{\left|\mathcal{S}_{k}\right|} \sum_{\sigma \in \mathcal{S}_{k}} \operatorname{sgn}(\sigma) \Lambda_{k}\left(u_{\sigma(1)} \otimes \cdots \otimes u_{\sigma(k)}\right) \\
& =\frac{1}{\left|\mathcal{S}_{k}\right|} \sum_{\sigma \in \mathcal{S}_{k}} \operatorname{sgn}(\sigma) u_{\sigma(1)} \wedge \cdots \wedge u_{\sigma(k)} \\
& =\frac{1}{\left|\mathcal{S}_{k}\right|} \sum_{\sigma \in \mathcal{S}_{k}} \operatorname{sgn}(\sigma)\left(\frac{1}{\left|\mathcal{S}_{k}\right|} \sum_{\varepsilon \in \mathcal{S}_{k}} \operatorname{sgn}(\varepsilon) u_{\varepsilon(\sigma(1))} \otimes \cdots \otimes u_{\varepsilon(\sigma(k)))}\right) \\
& =\frac{1}{\left|\mathcal{S}_{k}\right|} \sum_{\sigma \in \mathcal{S}_{k}}\left(\frac{1}{\left|\mathcal{S}_{k}\right|} \sum_{\varepsilon \in \mathcal{S}_{k}} \operatorname{sgn}(\varepsilon \sigma) u_{\varepsilon \sigma(1)} \otimes \cdots \otimes u_{\varepsilon \sigma(k)}\right) \\
& =\frac{1}{\left|\mathcal{S}_{k}\right|} \sum_{\sigma \in \mathcal{S}_{k}} u_{1} \wedge \cdots \wedge u_{k}=u_{1} \wedge \cdots \wedge u_{k}
\end{aligned}
$$

que aliado da (P.44) e arbitrariedade dos $u_{i} \in \mathfrak{H}$, caracteriza $\Lambda_{k}$ como um projetor ortogonal. Com efeito, pois se $\Lambda_{k}^{2}, \Lambda_{k}$ e $\Lambda_{k}^{*}$ coincidem nos tensores puros, devem coincidir em $\left\langle\otimes^{k} \mathfrak{H}\right\rangle$ por linearidade, e logo em $\mathfrak{H}^{\otimes k}$ por continuidade. De modo análogo, mostra-se que para toda $k$-upla $u_{1}, \ldots, u_{k} \in \mathfrak{H}$ :

- $u_{\sigma(1)} \odot \cdots \odot u_{\sigma(k)}=u_{1} \odot \cdots \odot u_{k} \quad \forall \sigma \in \mathcal{S}_{k}$.

- Dado uma $m$-upla de escalares $\alpha_{1}, \ldots, \alpha_{m} \in \mathbb{C}$ e outra de vetores $v_{1}, \ldots, v_{m} \in \mathfrak{H}$, tem-se

$$
u_{1} \odot \cdots \odot u_{i-1} \odot\left(\sum_{j=1}^{m} \alpha_{j} v_{j}\right) \odot u_{i+1} \odot \cdots \odot u_{k}=\sum_{j=1}^{m} \alpha_{j} \cdot\left(u_{1} \odot \cdots u_{i-1} \odot v_{j} \odot u_{i+1} \odot \cdots \odot u_{k}\right)
$$

- Existe um, e somente um operador $\Gamma_{k} \in \mathcal{B}\left(\mathfrak{H}^{\otimes k}\right)$ satisfazendo $\Gamma_{k}\left(w_{1} \otimes \cdots \otimes w_{k}\right)=w_{1} \odot \cdots \odot w_{k}$ para toda e qualquer $k$-upla de vetores $w_{1}, \ldots, w_{k} \in \mathfrak{H}$.

- $\Gamma_{k}$ é um projetor ortogonal.

- Por último mas não menos importante, tem-se para toda $k$-upla $w_{1}, \ldots, w_{k} \in \mathfrak{H}$ que

$$
\begin{aligned}
\left\langle u_{1} \odot \cdots \odot u_{k}, w_{1} \odot \cdots \odot w_{k}\right\rangle_{\mathfrak{H} \otimes k} & =\left\langle u_{1} \odot \cdots \odot u_{k}, w_{1} \otimes \cdots \otimes w_{k}\right\rangle_{\mathfrak{H} \otimes k} \\
& =\left\langle u_{1} \otimes \cdots \otimes u_{k}, w_{1} \odot \cdots \odot w_{k}\right\rangle_{\mathfrak{H} \otimes k} \\
& =(1 / k) ! \operatorname{Per}^{2}\left(\left\langle u_{i}, w_{j}\right\rangle_{\mathfrak{H}}\right)_{i, j=1}^{k}
\end{aligned}
$$


Se $G$ é um subconjunto de $\mathfrak{H}$ qualquer, convém denotar

$$
\wedge^{k} G \doteq\left\{u_{1} \wedge \cdots \wedge u_{k}: u_{1}, \ldots, u_{k} \in G\right\} \quad \odot^{k} G \doteq\left\{u_{1} \odot \cdots \odot u_{k}: u_{1}, \ldots, u_{k} \in G\right\}
$$

Definição 45 (Espaço Fermiônico e Bosônico de Muitos Corpos). O espaço de $k \geq 2$ férmions sobre um espaço de Hilbert separável $\mathfrak{H}$ é definido como

$$
\operatorname{Alt}^{k}(\mathfrak{H}) \doteq \operatorname{Im}\left(\Lambda_{k}\right)
$$

e o espaço de $k$ bósons por

$$
\operatorname{Sym}^{k}(\mathfrak{H}) \doteq \operatorname{Im}\left(\Gamma_{k}\right)
$$

Embora o espaço de $k$ férmions (respec. bósons) seja batizado na literatura matemática como espaço dos tensores alternados (respec. simétricos) de ordem $k$, neste contexto se preza por uma nomenclatura que remeta ao conteúdo físico imbuído neste conceito, e daí nossa terminologia. Dado ainda que as simetrias envolvendo partículas idênticas só se manifestam em sistemas com duas ou mais destas, convencionamos

$$
\operatorname{Alt}^{1}(\mathfrak{H})=\operatorname{Sym}^{1}(\mathfrak{H})=\mathfrak{H}
$$

como sendo o espaço de um bóson ou férmion, e de modo similar,

$$
\mathfrak{H}^{\otimes 0}=\operatorname{Alt}^{0}(\mathfrak{H})=\operatorname{Sym}^{0}(\mathfrak{H})=\mathbb{C}
$$

para designar o espaço ausente de partículas, isto é, o vácuo quântico. Afim de manter as notações inclusive nos casos de uma ou nenhuma partícula, pomos $\Lambda_{1}=\Gamma_{1} \doteq \mathbb{1}$ e $\Lambda_{0}=\Gamma_{0} \doteq \mathbb{1}$.

Aqui vai alguns fatos úteis acerca desta ultima definição:

Proposição 46. Seja $\mathfrak{H}$ um espaço de Hilbert separável, $\Omega \doteq\left\{e_{i}\right\}_{i=1}^{\operatorname{dim}(\mathfrak{H})}$ uma base ortonormal, $k \geq 2$ $e$

$$
\mathcal{E} \doteq \sqrt{k !}\left\{e_{i_{1}} \wedge \cdots \wedge e_{i_{k}}: 1 \leq i_{1}<\cdots<i_{k} \leq \operatorname{dim}(\mathfrak{H})\right\}
$$

São verdadeiras as seguintes afirmações:

a) Os tensores alternados puros geram um subespaço denso em $\operatorname{Alt}^{k}(\mathfrak{H})$.

b) Se $k \geq \operatorname{dim}(\mathfrak{H})$, então $\operatorname{Alt}^{k}(\mathfrak{H})=\{0\}$.

c) $\mathcal{E}$ forma uma base ortonormal de $\mathrm{Alt}^{k}(\mathfrak{H})$.

Demonstração. Dado que os tensores puros convencionais geram um subespaço denso em $\mathfrak{H}^{\otimes k}$ e $\Lambda_{k}$ é linear contínua, segue a). Adiante, se o valor de $k$ excede a dimensão do espaço ambiente, anula-se todo tensor alternado puro (e portanto o espaço de $k$ férmions na íntegra) por que qualquer $k$-upla de vetores será inevitavelmente LD. Na sequência, se $\left(i_{1}, \ldots, i_{k}\right)$ e $\left(j_{1}, \ldots, j_{k}\right)$ são duas $k$-listas ordenadas distintas e

\footnotetext{
${ }^{2} \mathrm{O}$ permanente de uma matriz $A \doteq\left(a_{i j}\right)_{i, j=1}^{k} \in \mathrm{M}_{k}(\mathbb{C})$ é definido como $\operatorname{Per}(A) \doteq \sum_{\sigma \in \mathcal{S}_{k}} a_{1 \sigma(1)} \cdots a_{k \sigma(k)}$, ou seja, é na essência o determinante de $A$, só que sem as trocas de sinais referente a paridade de cada permutação de $\mathcal{S}_{k}$.
} 
cujas entradas não excedam a dimensão de $\mathfrak{H}$, ao menos um dos $i_{p}$ difere de todo $j_{q}{ }^{3}$, e portanto alguma coluna da matriz $\left(\left\langle e_{i_{p}}, e_{j_{q}}\right\rangle_{\mathfrak{H}}\right)_{p, q=1}^{k} \in \mathrm{M}_{k}(\mathbb{C})$ encontra-se recheada de zeros. Isso anula seu determinante, e por consequência

$$
\left\langle e_{i_{1}} \wedge \cdots \wedge e_{i_{k}}, e_{j_{1}} \wedge \cdots \wedge e_{j_{k}}\right\rangle_{\mathfrak{H}^{\otimes k}}=\frac{1}{\left|\mathcal{S}_{k}\right|} \operatorname{det}\left(\left\langle e_{i_{p}}, e_{j_{q}}\right\rangle_{\mathfrak{H}}\right)_{p, q=1}^{k}=0
$$

Além disso,

$$
\|\left. e_{i_{1}} \wedge \cdots \wedge e_{i_{k}}\right|_{\mathfrak{H}^{\otimes k}} ^{2}=\frac{1}{\left|\mathcal{S}_{k}\right|} \operatorname{det}\left(\left\langle e_{i_{p}}, e_{i_{q}}\right\rangle_{\mathfrak{H}}\right)_{p, q=1}^{k}=\frac{1}{k !} \operatorname{det}\left(\delta_{p q}\right)_{p, q=1}^{k}=\frac{1}{k !}
$$

o que efetiva a ortonormalidade do conjunto $\mathcal{E}$. Enfim, basta mostrar que o subespaço gerado por $\mathcal{E}$ é denso nos tensores alternados puros. De fato, dado uma $k$-upla de vetores $u_{1}, \ldots, u_{k}$ em $\mathfrak{H}$, a continuidade ${ }^{4} \mathrm{e}$ multilinearidade do produto tensorial nos proporciona

$$
\begin{aligned}
u_{1} \otimes \cdots \otimes u_{k} & =\|\cdot\|_{\mathfrak{H}^{\otimes k}}-\lim _{m \rightarrow \infty}\left(\sum_{n_{1}=1}^{m}\left\langle e_{n_{1}}, u_{1}\right\rangle_{\mathfrak{H}} e_{n_{1}}\right) \otimes \cdots \otimes\left(\sum_{n_{k}=1}^{m}\left\langle e_{n_{k}}, u_{k}\right\rangle_{\mathfrak{H}} e_{k_{n}}\right) \\
& =\|\cdot\|_{\mathfrak{H}^{\otimes k}}-\lim _{m \rightarrow \infty} \sum_{n_{1}=1}^{m} \cdots \sum_{n_{k}=1}^{m}\left\langle e_{n_{1}}, u_{1}\right\rangle_{\mathfrak{H}} \cdots\left\langle e_{n_{k}}, u_{k}\right\rangle_{\mathfrak{H}} e_{n_{1}} \otimes \cdots \otimes e_{n_{k}}
\end{aligned}
$$

e que às custas da continuidade e linearidade de $\Lambda_{k}$, origina

$$
u_{1} \wedge \cdots \wedge u_{k}=\|\cdot\|_{\mathfrak{H} \otimes k} \lim _{m \rightarrow \infty} \sum_{n_{1}=1}^{m} \cdots \sum_{n_{k}=1}^{m}\left\langle e_{n_{1}}, u_{1}\right\rangle_{\mathfrak{H}} \cdots\left\langle e_{n_{k}}, u_{k}\right\rangle_{\mathfrak{H}} e_{n_{1}} \wedge \cdots \wedge e_{n_{k}} \in \overline{\left\langle\wedge^{k} \Omega\right\rangle}
$$

Embora $\mathcal{E}$ esteja contido propriamente em $\wedge^{k} \Omega$, note que seus subespaços gerados coincidem pois todo vetor do segundo (inclusive os excedentes) é colinear a algum vetor do primeiro, de novo pela (P.44).

Encerramos esta seção com a exposição de uma classe de operadores que exercem um papel central no formalismo da segunda quantização.

De fato, se $\Omega$ é uma base ortonormal de um espaço de Hilbert separável $\mathfrak{H}$, então para cada natural $k$ e vetor $u \in \mathfrak{H}$ que fixamos, existe um único operador linear

$$
c_{k}^{-}(u):\left\langle\otimes^{k} \Omega\right\rangle \leq \mathfrak{H}^{\otimes k} \longrightarrow \mathfrak{H}^{\otimes(k-1)}
$$

satisfazendo

$$
c_{k}^{-}(u)\left(e_{1} \otimes \cdots \otimes e_{k}\right)=u^{*}\left(e_{1}\right) e_{2} \otimes \cdots \otimes e_{k}
$$

para toda $k$-upla de vetores $e_{1}, \ldots, e_{k} \in \Omega$. Assim definido, $c_{k}^{-}(u)$ é limitado pois preserva a ortogonalidade de $\otimes^{k} \Omega$, além de verificar a estimativa

$$
\left\|\left.c_{k}^{-}(u)\left(e_{1} \otimes \cdots \otimes e_{k}\right)\right|_{\mathfrak{H}^{\otimes(k-1)}}=\left|u^{*}\left(e_{1}\right)\right| \cdot\right\| e_{2}||_{\mathfrak{H}} \cdots\left\|e_{k}\right\|_{\mathfrak{H}} \leq\left\|u^{*}\right\|_{\text {op }}=\|u\|_{\mathfrak{H}}
$$

\footnotetext{
${ }^{3}$ Do contrário essas $k$-listas contemplariam, a menos da ordem, os mesmos números naturais. Oras, se só existe uma maneira de ordenar um mesmo pacote de números, elas teriam de ser a mesma.

${ }^{4}$ Claro, pois como a norma do produto tensorial de uma $k$-upla de vetores é o produto das normas individuais de cada vetor, sua convergência recai sobre a convergência de cada parâmetro.
} 
também nos vetores de $\otimes^{k} \Omega$. Logo se estende univocamente a um operador limitado definido na totalidade de $\mathfrak{H}^{\otimes k}$ e igualmente denotado por $c_{k}^{-}(u)$. Como das outras vezes, mostra-se que a ação de $c_{k}^{-}(u)$ dada acima persiste num tensor puro qualquer, o que em particular revela sua autonomia com relação a escolha da base $\Omega$ fixada no início.

Supondo agora que $k \in \mathbb{N}_{0}$, ponha $c_{k}^{+}(u) \doteq c_{k+1}^{-}(u)^{*}$. Para toda $(2 k+1)$-upla $u_{1}, \ldots, u_{k}, w_{0}, \ldots, w_{k} \in \mathfrak{H}$, tem se

$$
\begin{aligned}
\left\langle u_{1} \otimes \cdots \otimes u_{k}, c_{k+1}^{-}(u)\left(w_{0} \otimes \cdots \otimes w_{k}\right)\right\rangle_{\mathfrak{H}^{\otimes k}} & =u^{*}\left(w_{0}\right)\left\langle u_{1} \otimes \cdots \otimes u_{k}, w_{1} \otimes \cdots \otimes w_{k}\right\rangle_{\mathfrak{H} \otimes k} \\
& =\left\langle u, w_{0}\right\rangle_{\mathfrak{H}} \cdot\left\langle u_{1}, w_{1}\right\rangle_{\mathfrak{H}} \cdots\left\langle u_{k}, w_{k}\right\rangle_{\mathfrak{H}} \\
& =\left\langle u \otimes u_{1} \otimes \cdots \otimes u_{k}, w_{1} \otimes \cdots \otimes w_{k}\right\rangle_{\mathfrak{H}} \otimes(k+1)
\end{aligned}
$$

e portanto $c_{k}^{+}(u)\left(u_{1} \otimes \cdots \otimes u_{k}\right)=u \otimes u_{1} \otimes \cdots \otimes u_{k}$. Depois de tanto preparo, eis o ponto a que queríamos chegar:

Definição 47 (Operador de Criação e Aniquilação Fermiônico). Seja $\mathfrak{H}$ um espaço de Hilbert separável. O operador de aniquilação fermiônico do estado $u \in \mathfrak{H}$ na ordem $k \in \mathbb{N}$ é definido por

$$
a_{k}(u) \doteq \sqrt{k} \Lambda_{k-1} c_{k}^{-}(u) \Lambda_{k}
$$

e o de criação na ordem $k \in \mathbb{N}_{0}$ por

$$
a_{k}^{\dagger}(u) \doteq a_{k+1}(u)^{*}=\sqrt{k+1} \Lambda_{k+1} c_{k}^{+}(u) \Lambda_{k}
$$

Antes de mais nada, vejamos como esses operadores agem no produto tensorial alternado de uma $k$-upla de vetores $u_{1}, \ldots, u_{k} \in \mathfrak{H}, \operatorname{com} k \geq 1$. Com efeito, denotando $u_{k+1} \equiv u$ e pondo

$$
\theta_{\sigma}(i)= \begin{cases}\sigma(i) & , \quad 1 \leq i \leq k \\ k+1 & , i=k+1\end{cases}
$$

para cada $\sigma \in \mathcal{S}_{k}$, computamos

$$
\begin{aligned}
a_{k}^{\dagger}(u)\left(u_{1} \wedge \cdots \wedge u_{k}\right) & =\sqrt{k+1} \cdot \frac{1}{\left|\mathcal{S}_{k}\right|} \sum_{\sigma \in \mathcal{S}_{k}} \operatorname{sgn}(\sigma) u \wedge u_{\sigma(1)} \wedge \cdots \wedge u_{\sigma(k)} \\
& =\sqrt{k+1} \cdot \frac{1}{\left|\mathcal{S}_{k}\right|} \sum_{\sigma \in \mathcal{S}_{k}} \operatorname{sgn}\left(\theta_{\sigma}\right) u_{\theta_{\sigma}(k+1)} \wedge u_{\theta_{\sigma}(1)} \wedge \cdots \wedge u_{\theta_{\sigma}(k)} \\
& =\sqrt{k+1} \cdot \frac{1}{\left|\mathcal{S}_{k}\right|} \sum_{\sigma \in \mathcal{S}_{k}} u_{k+1} \wedge u_{1} \wedge \cdots \wedge u_{k} \\
& =\sqrt{k+1} u \wedge u_{1} \wedge \cdots \wedge u_{k}
\end{aligned}
$$

visto que $\theta_{\sigma}$ tem (por construção) mesma paridade que $\sigma^{5}$. Já a ação do operador de aniquilação do estado

\footnotetext{
${ }^{5}$ Se $\sigma$ se decompõe no produto $\sigma=\tau_{1} \cdots \tau_{l}$, onde cada $\tau_{i}$ é um 2-ciclo em $\mathcal{S}_{k}$, então $\theta_{\sigma}=\mathcal{T}_{1} \cdots \mathcal{T}_{l}$, em que $\mathcal{T}_{i}$ é um 2-ciclo em $\mathcal{S}_{k+1}$ que fixa $k+1$, e coincide $\operatorname{com} \tau_{i}$ nos demais.
} 
$u$ nessa mesma $k$-upla de vetores é dada por

$$
\begin{aligned}
a_{k}(u)\left(u_{1} \wedge \cdots \wedge u_{k}\right) & =\sqrt{k} \cdot \frac{1}{\left|\mathcal{S}_{k}\right|} \sum_{\sigma \in \mathcal{S}_{k}} \operatorname{sgn}(\sigma) u^{*}\left(u_{\sigma(1)}\right) u_{\sigma(2)} \wedge \cdots \wedge u_{\sigma(k)} \\
& =\sqrt{k} \cdot \frac{1}{\left|\mathcal{S}_{k}\right|} \sum_{i=1}^{k} u^{*}\left(u_{i}\right) \sum_{\sigma \in \mathcal{S}_{k}: \sigma(1)=i} \operatorname{sgn}(\sigma) u_{\sigma(2)} \wedge \cdots \wedge u_{\sigma(n)} \\
& =\frac{1}{\sqrt{k}} \sum_{i=1}^{k}(-1)^{i+1}\left\langle u, u_{i}\right\rangle_{\mathfrak{H}} u_{1} \wedge \cdots \wedge \widehat{u}_{i} \wedge \cdots \wedge u_{k}
\end{aligned}
$$

Quando $\mathfrak{H}$ representa o espaço de estados de um determinado férmion, $\mathrm{Alt}^{k}(\mathfrak{H})$ descreve o espaço de estados do sistema composto por $k$ desses férmions. Para uma dada $k$-upla de vetores $u_{1}, \ldots, u_{k} \in \mathfrak{H}$ dois a dois ortogonais ou colineares, interpretamos o estado composto $u_{1} \wedge \cdots \wedge u_{k}$ como aquele em que um dos férmions se encontra no estado $u_{1}$, outro no estado $u_{2}$, e assim por diante. Contudo, advertimos o leitor de que estímulos atribuídos a um dos férmions será respondido pelo sistema como um todo, ou seja, não se tem mais uma descrição individual de cada férmion do sistema sem que a sua contra-parte seja mencionada. Com efeito, este fato está relacionado com a indistinguibilidade de férmions e entrelaçamento (ou emaranhamento) quântico a ela relacionado. Feitas estas cosiderações, veja então que $a_{k}^{\dagger}(u)$ e $a_{k}(u)$ foram concebidos para injetar e subtrair (respectivamente) férmions com estado $u$ no sistema em questão, e daí a terminologia para estes operadores.

Com essas definições, verificam-se as famosas relações canônicas de anticomutação (CAR)

$$
\{a(f), a(g)\}=\left\{a^{\dagger}(f), a^{\dagger}(g)\right\}=0 \quad, \quad\left\{a(f), a^{\dagger}(g)\right\}=\langle f \mid g\rangle
$$

escritas desta maneira apenas como um reforço visual. Nos poupamos desta tarefa pois estamos interessados num problema de três corpos em que não se almeja todo esplendor e glória de tais relações. Por fim, sempre que tentamos injetar dois férmions de mesmo estado no sistema, acontece

$$
\begin{aligned}
a_{k+1}^{\dagger}(u) a_{k}^{\dagger}(u)\left(u_{1} \wedge \cdots \wedge u_{k}\right) & =\sqrt{k+1} \cdot a_{k+1}^{\dagger}(u)\left(u \wedge u_{1} \wedge \cdots \wedge u_{k}\right) \\
& =\sqrt{(k+1)(k+2)} \cdot u \wedge u \wedge u_{1} \wedge \cdots \wedge u_{k} \\
& =0
\end{aligned}
$$

resguardando um importantíssimo postulado físico:

"Dois ou mais férmions idênticos não podem ocupar o mesmo estado dentro de um sistema quântico simultaneamente."

Também conhecido como Princípio de Exclusão de Pauli, devido ao físico austríaco Wolfgang Pauli ${ }^{6}$, ele explica uma grande variedade de fenômenos físicos e químicos que se observam experimentalmente, como o fato dos elétrons se empilharem em estados quânticos diversos no interior de um átomo, provocando uma enorme gama de configurações moleculares que temos hoje, e assim possibilitando a química e vidas complexas possíveis.

${ }^{6}$ Wolfgang Ernst Pauli, $\odot(25 / 04 / 1900)-\dagger(15 / 12 / 1958)$ 
Definição 48 (Operador de Criação e Aniquilação Bosônico). Seja $\mathfrak{H}$ um espaço de Hilbert separável. O operador de aniquilação bosônico do estado $u \in \mathfrak{H}$ na ordem $k \in \mathbb{N}$ é definido por

$$
b_{k}(u) \doteq \sqrt{k} \Gamma_{k-1} c_{k}^{-}(u) \Gamma_{k}
$$

e o de criação na ordem $k \in \mathbb{N}_{0}$ por

$$
b_{k}^{\dagger}(u) \doteq b_{k+1}(u)^{*}=\sqrt{k+1} \Gamma_{k+1} c_{k}^{+}(u) \Gamma_{k}
$$

De modo análogo, mostra-se que para toda $k$-upla $u_{1}, \ldots, u_{k} \in \mathfrak{H}$ :

- Os tensores simétricos puros geram um subespaço denso em $\operatorname{Sym}^{k}(\mathfrak{H})$.

- Se $\Omega=\left\{e_{i}\right\}_{i=1}^{\operatorname{dim}(\mathfrak{H})}$ é uma base ortonormal de $\mathfrak{H}$,

$$
\mathcal{G} \doteq \sqrt{k !}\left\{e_{i_{1}} \odot \cdots \odot e_{i_{k}}: 1 \leq i_{1}<\cdots i_{k} \leq \operatorname{dim}(\mathfrak{H})\right\}
$$

é base ortonormal de $\operatorname{Sym}^{k}(\mathfrak{H})$.

- $b_{k}^{\dagger}(u)\left(u_{1} \odot \cdots \odot u_{k}\right)=\sqrt{k+1} u \odot u_{1} \odot \cdots \odot u_{k}$

- $b_{n}(u)\left(u_{1} \odot \cdots \odot u_{k}\right)=\frac{1}{\sqrt{k}} \sum_{i=1}^{k}\left\langle u, u_{i}\right\rangle_{\mathfrak{H}} u_{1} \odot \cdots \odot \widehat{u_{i}} \odot \cdots \odot u_{k}$

- Com excessão do princípio de exclusão de Pauli, vale considerações físicas análoga para os bósons.

- Se $k \geq 2$, então $\operatorname{Sym}^{k}(\mathfrak{H}) \perp \mathrm{Alt}^{k}(\mathfrak{H})$.

Com base em tudo o que já sabemos, basta mostrar que todo tensor alternado puro é ortogonal a todo tensor simétrico puro. Claramente, pois

$$
\left\langle u_{1} \wedge \cdots \wedge u_{k}, w_{1} \odot \cdots \odot w_{k}\right\rangle_{\mathfrak{H}^{\otimes k}}=\frac{1}{\left|\mathcal{S}_{k}\right|^{2}} \sum_{\sigma \in \mathcal{S}_{k}} \operatorname{sgn}(\sigma) \operatorname{det}\left(\left\langle u_{i}, w_{j}\right\rangle_{\mathfrak{H}}\right)_{i, j=1}^{k} \stackrel{7}{=} 0
$$

Definição 49 (Operador Número de Partículas Fermiônicas e Bosônicas). Seja $\mathfrak{H}$ um espaço de Hilbert separável. O operador número de partículas fermiônicas no estado $u \in \mathfrak{H}$ de ordem $k \in \mathbb{N}$ é definido como

$$
n_{k}^{-}(u) \doteq a_{k-1}^{\dagger}(u) a_{k}(u)
$$

e o operador número de partículas bosônicas em $u$ de ordem $k$ por

$$
n_{k}^{+}(u) \doteq b_{k-1}^{\dagger}(u) b_{k}(u)
$$

\footnotetext{
${ }^{7}$ Confira o bestiário de resultados ao final do trabalho.
} 
Para uma dada $k$-upla de vetores $u_{1}, \ldots, u_{k} \in \mathfrak{H}$, tem-se

$$
\begin{aligned}
n_{k}^{+}(u)\left(u_{1} \odot \cdots \odot u_{k}\right) & =\sum_{i=1}^{k}\left\langle u, u_{i}\right\rangle_{\mathfrak{H}} u \odot u_{1} \odot \cdots \odot u_{k} \\
& =\sum_{i=1}^{k}\left\langle u, u_{i}\right\rangle_{\mathfrak{H}} u_{1} \odot \cdots \odot u_{i-1} \odot u \odot u_{i+1} \odot \cdots \odot u_{k}
\end{aligned}
$$

e supondo agora que $u, u_{1}, \ldots, u_{k}$ são dois a dois ortogonais ou colineares, obtemos

$$
n_{k}^{+}(u)\left(u_{1} \odot \cdots \odot u_{k}\right)=p \cdot u_{1} \odot \cdots \odot u_{k}
$$

onde $0 \leq p \leq k$ quantifica quantos dos $u_{i}$ são iguais à $u$. Com isso vemos que $n_{k}^{+}(u)$ funciona como uma espécie de contador, informando quantos bósons estão ocupando o estado $u$ quando o sistema se encontra no estado $u_{1} \odot \cdots \odot u_{k}$. No caso dos férmions, devemos lembrar que os valores de $p$ sempre ficam restritos a 0 e 1 , dado que repetições não são admitidas.

Na segunda parte do trabalho,

$$
\|u\|_{\wedge} \doteq \sqrt{k !}\|u\|_{\mathfrak{H}^{\otimes k}}, \quad u \in \mathrm{Alt}^{k}(\mathfrak{H}) \quad\|v\|_{\odot} \doteq \sqrt{k !}\|v\|_{\mathfrak{H}^{\otimes k}}, \quad v \in \operatorname{Sym}^{k}(\mathfrak{H})
$$

passarão a ser as normas utilizadas, por conveniência, para os espaços de $k$ férmions e bósons sobre $\mathfrak{H}$, respectivamente. Todos os fenômenos de natureza topológica que provamos permanecem válidos, dado que essas normas são (por construção) equivalentes às antiga.

\subsection{Operadores de Onda de Kato}

"A matemática não apenas descreve e explica a natureza, mas também faz parte do jogo interno entre a natureza e nós mesmos."

Werner Heisenberg 8

Definição 50 (Grupo Unitário Fortemente Contínuo). Uma família $\{U(t): t \in \mathbb{R}\}$ de operadores lineares agindo em um espaço de Hilbert $\mathfrak{H}$ diz-se um grupo unitário (à um parâmetro real) quando cumpre as seguintes condições:

a) $U(t)$ é unitário para todo $t \in \mathbb{R}$

b) $U(t+s)=U(t) U(s) \quad \forall t, s \in \mathbb{R}$

Se além disso, a função $\mathbb{R} \ni t \longmapsto U(t) \varphi \in \mathfrak{H}$ for contínua para todo $\varphi \in \mathfrak{H}$, dizemos que esse grupo unitário é fortemente contínuo.

As condições acima implicam, em particular, que $\{U(t)\}_{t \in \mathbb{R}}$ é de fato um grupo (abeliano) no sentido convencional: A composição de operadores define uma operação binária comutativa na família em questão por conta de b); além disso

${ }^{8}$ Werner Karl Heisenberg, $\bigcirc(05 / 12 / 1901)-\dagger(01 / 02 / 1976)$ 


$$
U\left(t_{0}\right) \psi=U\left(0+t_{0}\right) \psi=U(0) U\left(t_{0}\right) \psi \quad \forall \psi \in \mathfrak{H}
$$

e que graças a sobrejetividade de $U\left(t_{0}\right)$, equivale a

$$
\varphi=U(0) \varphi \quad \forall \varphi \in \mathfrak{H}
$$

e portanto $U(0)=\mathbb{1}$. Finalmente, $U(-t)$ desempenha o papel do inverso de $U(t)$ para todo $t \in \mathbb{R}$.

Aliás, para que um grupo unitário $\{U(t)\}_{t \in \mathbb{R}}$ seja fortemente contínuo, é suficiente que a função $U(\cdot) \varphi$ seja contínua apenas na origem. Com efeito, pois dado $\varepsilon>0$ e fornecido um $\delta>0$ correspondente, isto é, de modo a

$$
s \in \mathbb{R}:|s|<\delta \Longrightarrow\|U(s) \varphi-\varphi\|_{\mathfrak{H}}<\varepsilon
$$

segue da unitariedade de $U\left(t_{0}\right)$ que

$$
t \in \mathbb{R}:\left|t-t_{0}\right|<\delta \Longrightarrow\left\|U(t) \varphi-U\left(t_{0}\right) \varphi\right\|_{\mathfrak{H}}=\left\|U\left(-t_{0}\right)\left[U(t) \varphi-U\left(t_{0}\right) \varphi\right]\right\|_{\mathfrak{H}}=\left\|U\left(t-t_{0}\right) \varphi-\varphi\right\|_{\mathfrak{H}}<\varepsilon
$$

Exemplo 51. Seja $\mathfrak{H}$ um espaço de Hilbert e $A \in \mathcal{B}(\mathfrak{H})$ autoadjunto. Para cada $\psi \in \mathfrak{H}$ que fixamos, $\omega(t) \doteq e^{\imath t A} \psi$ é solução do problema linear autônomo de valor inicial

$$
\left\{\begin{array}{l}
x^{\prime}=\imath A x \\
x(0)=\psi
\end{array}\right.
$$

Em particular, $\left\{e^{\imath t A}\right\}_{t \in \mathbb{R}}$ constitui um grupo unitário fortemente contínuo.

Para cada $t \in \mathbb{R}$, ponha $f_{t}(\lambda) \doteq e^{\imath \lambda t}$. Dado que o cálculo boreliano de $A$ é um homomorfismo de álgebra involutiva e as $f_{t}$ verificam $f_{r} f_{s}=f_{r+s}$ e $\overline{f_{s}}=f_{-s}=f_{s}^{-1}$ para todo $r, s \in \mathbb{R}$,

$$
\left\{e^{\imath t A}\right\}_{t \in \mathbb{R}}=\Phi_{A}\left(\left\{f_{t}\right\}_{t \in \mathbb{R}}\right)
$$

cumpre as exigências que o define como um grupo unitário. Vejamos agora que $\omega$ confere a solução da equação diferencial linear proposta no enunciado. Com efeito, para todo $\lambda \in \mathbb{R}$, tem-se

$$
\left.\frac{\mathrm{d}\left(e^{\imath s \lambda}\right)}{\mathrm{d} s}\right|_{s=0}=\imath \lambda \Longrightarrow \lim _{s \rightarrow 0}\left|\frac{e^{\imath s \lambda}-1}{s}-\imath \lambda\right|=0
$$

bem como

$$
\left|\frac{e^{\imath s \lambda}-1}{s}-\imath \lambda\right| \leq{ }^{9} 2|\lambda| \in \mathrm{L}^{1}\left(\Omega_{\psi}\right) \quad \forall s \neq 0
$$

e por efeito do teorema da convergência dominada,

$$
\begin{aligned}
& \lim _{t \rightarrow t_{0}}\left\|\frac{\omega(t)-\omega\left(t_{0}\right)}{t-t_{0}}-\imath A \omega\left(t_{0}\right)\right\|_{\mathfrak{H}}^{2}=10 \lim _{t \rightarrow t_{0}}\left\|\frac{\omega\left(t-t_{0}\right)-\omega(0)}{t-t_{0}}-\imath A \omega(0)\right\|_{\mathfrak{H}}^{2}= \\
& \quad=\lim _{s \rightarrow 0}\left\|\frac{\omega(s)-\omega(0)}{s}-\imath A \omega(0)\right\|_{\mathfrak{H}}^{2}=\lim _{s \rightarrow 0}\left\|\frac{e^{\imath s A} \psi-\psi}{s}-\imath A \psi\right\|_{\mathfrak{H}}^{2}=\lim _{s \rightarrow 0} \int_{\mathbb{R}}\left|\frac{e^{\imath \lambda s}-1}{s}-\imath \lambda\right|^{2} \mathrm{~d} \Omega_{\psi}(\lambda)=0
\end{aligned}
$$

\footnotetext{
${ }^{9}$ Confira o apêndice A.
} 
Como $t_{0}$ é arbitrário, provamos que $\omega$ é diferenciável, valendo

$$
\omega^{\prime}(t)=\imath A \omega(t)
$$

Definição 52 (Gerador Infinitesimal). Seja $\mathfrak{H}$ um espaço de Hilbert. Dizemos que um operador autoadjunto $A \in \mathcal{B}(\mathfrak{H})$ é gerador infinitesimal de um grupo unitário $\{U(t)\}_{t \in \mathbb{R}}$ se para todo $t \in \mathbb{R}$, vale

$$
U(t)=e^{\imath t A}
$$

Note que o gerador infinitesimal de grupo unitário, quando existe, é único. De fato, se os $U(t)$ possuíssem dois geradores $A$ e $B$, concluiríamos

$$
\imath A \psi \stackrel{t \rightarrow 0}{\longleftarrow} \frac{e^{\imath t A} \psi-\psi}{t}=\frac{e^{\imath t B} \psi-\psi}{t} \stackrel{t \rightarrow 0}{\longrightarrow} \imath B \psi \quad \forall \psi \in \mathfrak{H}
$$

a partir do exemplo acima, e portanto $A=B$, pela unicidade do limite em espaços métricos.

$\mathrm{Na}$ realidade, o ilustre teorema de Stone garante que todo grupo unitário fortemente contínuo admite um gerador infinitesimal, e portanto (Ex.51) não se trata mais de um exemplo em particular, mas sim da forma geral desses grupos.

$\mathrm{Na}$ concepção moderna, um sistema quântico é descrito por um espaço de Hilbert $\mathfrak{H}$ cujos vetores representam seus estados e um grupo unitário fortemente contínuo $\{U(t)\}_{t \in \mathbb{R}}$ agindo nesse espaço. O Hamiltoniano referente a esse sistema é definido por $\hat{H} \doteq-\hbar A$, onde $A$ é o gerador infinitesimal dos $U(t)$. Em virtude do exemplo acima, perceba que a evolução temporal de cada estado $\varphi \in \mathfrak{H}$ conforme a dinâmica implementada pelo grupo unitário $\{U(t)\}_{t \in \mathbb{R}}$, ou seja,

$$
\Psi(t) \doteq U(t) \varphi=e^{\imath t A} \varphi=e^{-\frac{2 t}{\hbar} \hat{H}}, \quad t \in \mathbb{R}
$$

é governada pela célebre equação de Schrödinger ${ }^{11}$

$$
\imath \hbar \frac{\mathrm{d} \Psi}{\mathrm{d} t}(t)=\hat{H} \Psi(t)
$$

A teoria de espalhamento está relacionada a dois tipos de dinâmicas associadas a um mesmo sistema; a dinâmica interagente dada por um Hamiltoniano $H$ e a dinâmica livre dada por um segundo Hamiltoniano $H_{0}$ diferente do primeiro. Tipicamente, a dinâmica interagente é difícil de se resolver analiticamente, enquanto que a livre é mais tratável e conserva o momento das partes individuais do sistema. Porém, se a diferença entre essas dinâmicas for pequena em algum sentido, é razoável pensar que informações sobre o sistema quando sujeito a dinâmica livre possa nos fornecer informações sobre o sistema quando sujeito a dinâmica interagente. Neste sentido, a teoria de espalhamento pode ser vista como um tipo de teoria de perturbação.

Dizemos que um estado $\varphi \in \mathfrak{H}$ é assistoticamente livre num passado distante quando existe um estado $\varphi_{-} \in \mathfrak{H}$ tal que

$$
\lim _{t \rightarrow-\infty}\left\|e^{-\imath t H_{0}} \varphi_{-}-e^{-\imath t H} \varphi\right\|_{\mathfrak{H}}=0
$$

\footnotetext{
${ }^{10}$ Lembre-se de que $A$ comuta com $e^{-\imath t_{0} A}$ por que esse ultimo é cálculo funcional do primeiro.

${ }^{11}$ Erwin Rudolf Josef Alexander Schrödinger, O(12/08/1887) - †(04/01/1961)
} 
Em palavras, tais estados são aqueles cuja evolução por meio da dinâmica interagente num passado distante equiparáva-se a de outro estado devido à dinâmica livre. Equivalentemente ao limite acima, note que

$$
\lim _{t \rightarrow-\infty}\left\|e^{\imath t H} e^{-\imath t H_{0}} \varphi_{-}-\varphi\right\|_{\mathfrak{H}}=0
$$

de modo que a busca por estados que são assintoticamente livres num passado distante se reduz a existência de limites fortes (pontuais). Similarmente, define-se um estado assintoticamente livre num futuro distante, e com base nessa discussão que enunciamos a definição principal desta seção:

Definição 53 (Operador de Onda de Kato). Sejam A e B operadores limitados e autoadjuntos agindo nos espaços de Hilbert $\mathfrak{H}$ e $\mathcal{H}$, respectivamente. Os operadores de onda de Kato do par $(A, B)$ associado ao operador de identificação $J \in \mathcal{B}(\mathcal{H}, \mathfrak{H})$ são definidos por

$$
W^{ \pm}(A, B ; J) \psi \doteq \lim _{t \rightarrow \mp \infty} e^{\imath t A} J e^{-\imath t B} P_{a c}(B) \psi, \quad \psi \in \mathcal{H}
$$

quando esses limites existem.

Ao contrário do que sugere [RS79], faremos uma breve exposição da teoria de espalhamento em dois espaços devido a sua maior flexibilidade, e quando houver necessidade de $A$ e $B$ atuarem em um mesmo espaço de Hilbert, escreveremos $\Omega^{ \pm}(A, B) \equiv W^{ \pm}(A, B ; \mathbb{1})$ em concordância com a referida fonte.

Proposição 54. Sejam $A$ e $B$ operadores limitados e autoadjuntos agindo nos espaços de Hilbert $\mathfrak{H} e$ $\mathcal{H}$, respectivamente. Assuma também que $W^{ \pm}(A, B ; J)$ exista para um dado operador de identificação $J \in \mathcal{B}(\mathcal{H}, \mathfrak{H})$. São verdadeiras as seguintes afirmações:

a) $A W^{ \pm}(A, B ; J)=W^{ \pm}(A, B ; J) B$

b) $\operatorname{Im}\left(W^{ \pm}\right)$são subespaços A-invariantes de $\mathfrak{H}$.

Se J é uma isometria parcial com espaço inicial $\mathcal{H}_{\mathrm{ac}}(B)$, acrescenta-se:

c) $W^{ \pm}(A, B ; J)$ são isometrias parciais com espaço inicial $\mathcal{H}_{\mathrm{ac}}(B)$ e final $\operatorname{Im}\left(W^{ \pm}\right)$.

d) $\operatorname{Im}\left(W^{ \pm}\right) \subseteq \mathfrak{H}_{\mathrm{ac}}(A)$

Demonstração. Dado que o limite de função é invariante por translações, ainda é verdade

$$
\begin{aligned}
W^{ \pm}(A, B ; J) \psi=\lim _{t \mp \infty} e^{\imath(t+s) A} J e^{-\imath(t+s) B} P_{\mathrm{ac}}(B) \psi=\lim _{t \mp \infty} e^{\imath s A} e^{\imath t A} J e^{-\imath t B} P_{\mathrm{ac}}(B) e^{-\imath s B} \psi & = \\
& =e^{\imath s A} W^{ \pm}(A, B ; J) e^{-\imath s B} \psi
\end{aligned}
$$

sendo que na terceira passagem se sujeitamos a continuidade do operador $e^{\imath A s}$ para extraí-lo do limite, e na segunda que $P_{\text {ac }}(B)$ comuta com $e^{-\imath s B}$ por que comuta com o próprio $B$, vide (P.15). Aplicando a inversa de $e^{\imath A s}$ na equação resultante, obtemos

$$
e^{-\imath s A} W^{ \pm}(A, B ; J) \psi=W^{ \pm}(A, B ; J) e^{-\imath s B} \psi \quad \forall s \in \mathbb{R}
$$


que por ser válida em toda uma vizinhança aberta do ponto $s=0$, implica

$$
\begin{aligned}
-\imath A W^{ \pm}(A, B ; J) \psi=\left.\frac{\mathrm{d}}{\mathrm{d} s}\left(e^{-\imath s A} W^{ \pm}(A, B ; J) \psi\right)\right|_{s=0} & =\left.\frac{\mathrm{d}}{\mathrm{d} s}\left(W^{ \pm}(A, B ; J) e^{-\imath s B} \psi\right)\right|_{s=0}= \\
& =\left.W^{ \pm}(A, B ; J) \frac{\mathrm{d}}{\mathrm{d} s}\left(e^{-\imath s B} \psi\right)\right|_{s=0}=-\imath W^{ \pm}(A, B ; J) B \psi
\end{aligned}
$$

uma vez que a continuidade do operador $W^{ \pm}(A, B ; J)$ permite comutá-lo com a derivada (que se trata de um tipo de limite). Como $\psi \in \mathcal{H}$ é arbitrário, provamos a). Em sequência,

$$
\begin{aligned}
\varphi \in \operatorname{Im}\left(W^{ \pm}\right) \Longrightarrow \exists \psi \in \mathcal{H}: \varphi=W^{ \pm}(A, B ; J) \psi \Longrightarrow A \varphi=A W^{ \pm}(A, B ; J) \psi= \\
\quad=W^{ \pm}(A, B ; J) B \psi \in \operatorname{Im}\left(W^{ \pm}\right)
\end{aligned}
$$

donde b). Em frente as hipóteses adicionais quanto a $J$, computamos

$$
\left\|W^{ \pm}(A, B ; J) \psi\right\|_{\mathfrak{H}}=\lim _{t \rightarrow \mp \infty}\left\|e^{\imath t A} J e^{-\imath t B} P_{\mathrm{ac}}(B) \psi\right\|_{\mathfrak{H}}=\lim _{t \rightarrow \mp \infty}\left\|P_{\mathrm{ac}}(B) \psi\right\|_{\mathcal{H}}=\left\|P_{\mathrm{ac}}(B) \psi\right\|_{\mathcal{H}}
$$

pois observe que

$$
e^{-\imath t B} P_{\mathrm{ac}}(B) \psi=P_{\mathrm{ac}}(B) e^{-\imath t B} \psi \in \mathcal{H}_{\mathrm{ac}}(B)
$$

e claro, $\|\cdot\|_{\mathfrak{H}}$ é contínua. Oras, por um lado essas manipulações mostram que $W^{ \pm}(A, B ; J)$ e $P_{\text {ac }}(B)$ partilham do mesmo núcleo, e sendo assim

$$
\operatorname{Ker}\left(W^{ \pm}\right)^{\perp}=\operatorname{Ker}\left(P_{\mathrm{ac}}(B)\right)^{\perp}=\operatorname{Im}\left(P_{\mathrm{ac}}(B)\right)=\mathcal{H}_{\mathrm{ac}}(B)
$$

Por outro, que para todo vetor $\psi$ compreendido no espaço espectral absolutamente contínuo de $B$ verifica

$$
\left\|W^{ \pm}(A, B ; J) \psi\right\|_{\mathfrak{H}}=\|\psi\|_{\mathcal{H}}
$$

Finalmente, identificamos o espaço final dos $W^{ \pm}$por meio da identidade

$$
W^{ \pm}(A, B ; J)=W^{ \pm}(A, B ; J) P_{\mathrm{ac}}(B)
$$

e o item c) está provado. Por último mas não menos importante, ponha

$$
\begin{aligned}
U_{ \pm}: \mathcal{H}_{\mathrm{ac}}(B) & \longrightarrow \operatorname{Im}\left(W^{ \pm}\right) \\
\psi & \longmapsto W^{ \pm}(A, B ; J) \psi
\end{aligned}
$$

Refrisando que $\mathcal{H}_{\mathrm{ac}}(B)$ é um subespaço $B$-invariante de $\mathcal{H}$, vide (P.15), equacionamos

$$
A U_{ \pm} \psi=A W^{ \pm}(A, B ; J) \psi=W^{ \pm}(A, B ; J) B \psi=U_{ \pm} B \psi \quad \forall \psi \in \mathcal{H}_{\mathrm{ac}}(B)
$$

o que quer dizer

$$
A \uparrow \operatorname{Im}\left(W^{ \pm}\right) \sim B \uparrow \mathcal{H}_{\mathrm{ac}}(B)
$$

e consequentemente

$$
\mathfrak{H}_{\mathrm{ac}}(A) \cap \operatorname{Im}\left(W^{ \pm}\right)=U_{ \pm}\left(\mathcal{H}_{\mathrm{ac}}(B)\right)=\operatorname{Im}\left(W^{ \pm}\right)
$$


também por mérito da (P.15).

Proposição 55 (Regra da Cadeia). Sejam A, B e C operadores limitados e adutoadjuntos agindo nos espaços de Hilbert $\mathfrak{H}, \mathcal{H}$ e $\mathcal{H}$, respectivamente. Dado $J_{0} \in \mathcal{B}(\mathcal{H}, \mathfrak{H})$ e $J_{1} \in \mathcal{B}(\mathcal{H}, \mathcal{H})$ uma isometria parcial com espaço inicial $\mathcal{H}_{\mathrm{ac}}(C)$, suponha que tanto $W^{ \pm}\left(A, B ; J_{0}\right)$ quanto $W^{ \pm}\left(A, B ; J_{1}\right)$ existam. Então, $W^{ \pm}\left(A, C ; J_{1} J_{0}\right)$ existe e se relaciona com os demais por meio da seguinte fórmula:

$$
W^{ \pm}\left(A, C ; J_{0} J_{1}\right)=W^{ \pm}\left(A, B ; J_{0}\right) W^{ \pm}\left(B, C ; J_{1}\right)
$$

Demonstração. Visto que a imagem de $W^{ \pm}\left(B, C ; J_{1}\right)$ está contida no espaço absolutamente contínuo de $B$, efetua-se

$$
\lim _{t \rightarrow \mp \infty}\left(\mathbb{1}-P_{\mathrm{ac}}(B)\right) e^{\imath t B} J_{1} e^{-\imath t C} P_{\mathrm{ac}}(C) \varphi=\left(\mathbb{1}-P_{\mathrm{ac}}(B)\right) W^{ \pm}\left(B, C ; J_{1}\right) \varphi=0
$$

até por que $\mathbb{1}-P_{\mathrm{ac}}(B)$ é contínuo, e portanto imune ao limite. Aplicando um dos resultados suplementares que situamos no apêndice $A$, segue

$$
\lim _{t \rightarrow \mp \infty} e^{\imath t A} J_{0} e^{-\imath t B}\left(\mathbb{1}-P_{\mathrm{ac}}(B)\right) e^{\imath t B} J_{1} e^{-\imath t C} P_{\mathrm{ac}}(C) \varphi=0
$$

e pela mesma razão

$$
\lim _{t \rightarrow \mp \infty} e^{\imath t A} J_{0} e^{-\imath t B} P_{\text {ac }}(B) e^{\imath t B} J_{1} e^{-\imath t C} P_{\text {ac }}(C) \varphi=W^{ \pm}\left(A, B ; J_{0}\right) W^{ \pm}\left(B, C ; J_{1}\right) \varphi
$$

Enquanto isso, vale

$e^{\imath t A} J_{0} J_{1} e^{-\imath t C} P_{\mathrm{ac}}(C) \varphi=e^{\imath t A} J_{0} e^{-\imath t B} P_{\mathrm{ac}}(B) e^{\imath t B} J_{1} e^{-\imath t C} P_{\mathrm{ac}}(C) \varphi+e^{\imath t A} J_{0} e^{-\imath t B}\left(\mathbb{1}-P_{\mathrm{ac}}(B)\right) e^{\imath t B} J_{1} e^{-\imath t C} P_{\mathrm{ac}}(C) \varphi$ para todo $t \in \mathbb{R}$, e o resultado está provado.

A seguir, introduzimos uma classe muito especial de operadores de onda presente na teoria de KatoBirman:

Definição 56 (Operador de Onda de Kato Completo). Seja A e B operadores limitados e autoadjuntos agindo nos espaços de Hilbert $\mathfrak{H}$ e $\mathcal{H}$, respectivamente. Suponha que $W^{ \pm}(A, B ; J)$ exista para um dado operador de identificação $J \in \mathcal{B}(\mathcal{H}, \mathfrak{H})$. Dizemos que os operadores de onda de Kato do par $(A, B)$ são $J$-completos se

$$
\operatorname{Im}\left(W^{ \pm}(A, B ; J)\right)=\mathfrak{H}_{\mathrm{ac}}(A)
$$

Sob certas hipóteses, todo problema de completude se converte em um problema de existência. Este é o conteúdo que emana da seguinte proposição:

Proposição 57. Seja $A$ e $B$ operadores limitados e autoadjuntos agindo nos espaços de Hilbert $\mathfrak{H} e$ $\mathcal{H}$, respectivamente. Assuma ainda que para um dado operador de identificação unitário $J \in \mathcal{B}(\mathcal{H}, \mathfrak{H})$, $W^{ \pm}(A, B ; J)$ exista. Então, $W^{ \pm}(A, B ; J)$ é completo se, e somente se, $W^{ \pm}\left(B, A ; J^{*}\right)$ existe.

Demonstração. Suponha que $W^{ \pm}\left(B, A ; J^{*}\right)$ exista. Pela regra da cadeia, 


$$
P_{\mathrm{ac}}(A)=\Omega^{ \pm}(A, A)=W^{ \pm}\left(A, A ; J J^{*}\right)=W^{ \pm}(A, B ; J) W^{ \pm}\left(B, A ; J^{*}\right)
$$

e com isso todo $\psi \in \mathfrak{H}_{\text {ac }}(A)$ é imagem de $W^{ \pm}\left(B, A ; J^{*}\right) \psi$ por $W^{ \pm}(A, B ; J)$. Logo

$$
\mathfrak{H}_{\mathrm{ac}}(A) \subseteq \operatorname{Im}\left(W^{ \pm}(A, B ; J)\right)
$$

e que em função da (P.54), se trata de uma igualdade autêntica.

Reciprocamente, se $W^{ \pm}(A, B ; J)$ for completo, um dado $\varphi \in \mathfrak{H}_{\mathrm{ac}}(A)$ é imagem de algum $\psi \in \mathcal{H}$ por $W^{ \pm}(A, B ; J)$, e daí

$$
\begin{aligned}
\| e^{\imath t B} J^{*} e^{-\imath t A} P_{\mathrm{ac}}(A) \varphi-P_{\mathrm{ac}}(B) \psi & \left\|_{\mathcal{H}}=\right\| J^{*} e^{-\imath t A} \varphi-e^{-\imath t B} P_{\mathrm{ac}}(B) \psi \|_{\mathcal{H}}= \\
& =\left\|e^{-\imath t A} \varphi-J e^{-\imath t B} P_{\mathrm{ac}}(B) \psi\right\|_{\mathfrak{H}}=\left\|W^{ \pm}(A, B ; J) \psi-e^{\imath t A} J e^{-\imath t B} P_{\mathrm{ac}}(B) \psi\right\|_{\mathfrak{H}}
\end{aligned}
$$

Fazendo $t \rightarrow \mp \infty$, concretizamos

$$
\lim _{t \rightarrow \mp \infty} e^{\imath t B} J^{*} e^{-\imath t A} P_{\mathrm{ac}}(A) \varphi=P_{\mathrm{ac}}(B) \psi
$$

e com isso a existência de $W^{ \pm}\left(B, A ; J^{*}\right)$ em $\mathfrak{H}_{\text {ac }}(A)$, dado que $\varphi$ é arbitrário. Isso encerra a demonstração por que $W^{ \pm}\left(B, A ; J^{*}\right)$ também existe em $\mathfrak{H}_{\mathrm{ac}}(A)^{\perp}$ de maneira trivial.

Para encerrar, apresentamos dois resultados que asseguram a existência e completude de operadores de onda por meio de critérios extremamente práticos.

Teorema 58 (Pearson). Seja $\mathfrak{H}$ um espaço de Hilbert e $A, B \in \mathcal{B}(\mathfrak{H})$ autoadjuntos. Se $J \in \mathcal{B}(\mathfrak{H})$ satisfaz $A J-J B \in \mathscr{S}_{1}$, então $W^{ \pm}(A, B ; J)$ existe.

Demonstração. Ver [RS79].

Corolário 59 (Teorema de Kato-Rosenblum). Seja $\mathfrak{H}$ um espaço de Hilbert e $A, B \in \mathcal{B}(\mathfrak{H})$ autoadjuntos. Se $A-B \in \mathscr{S}_{1}(\mathfrak{H})$, então $\Omega^{ \pm}(A, B)$ existe e é completo.

Demonstração. Como $\mathscr{S}_{1}(\mathfrak{H})$ é um espaço vetorial, também vale

$$
B-A=-(A-B) \in \mathscr{S}_{1}(\mathfrak{H})
$$

de forma que a (P.57) é aplicável. 


\section{Parte II}

\section{Estudo Microscópico de Supercondutores com Alta Temperatura de Transição}





\section{Capítulo 4}

\section{Um Modelo de Pareamento em Supercondutores Não-Convencionais}

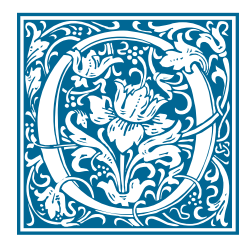

S SUPERCONDUTORES não convencionais atingem o estado de supercondutividade em temperaturas próximas a $77 \mathrm{~K}$, temperaturas críticas estas muito mais elevadas que as dos supercondutores usuais. Ao contrário dos supercondutores com baixa temperatura de transição, que são condutores em temperaturas mais elevadas que a temperatura crítica $T_{c}$ e explicados com sucesso pela teoria BCS, supercondutores não convencionais são isolantes em condições normais. Outra propriedade notória é a simetria $d$-wave no parâmetro de ordem observado experimentalmente, diferentemente dos supercondutores convencionais para os quais prevalece simetrias do tipo $s$-wave. A descoberta de supercondutores com alta temperatura de transição $\left(T_{c}\right)$ em 1986 representou um grande avanço experimental (prêmio Nobel de 1987), apesar das explicações teóricas à respeito deste novo estado da matéria não serem conclusivas até os dias de hoje.

Tudo isso revela que a diferença entre supercondutores convencionais e não convencionais não está associada única e exclusivamente a diferença de temperatura crítica, do mesmo modo que materiais diferentes possuem pontos de fusão diferentes, e por isso o entendimento de um modelo microscópico geral para esta classe de materiais pode revelar mecanismos que expliquem as propriedades que observamos experimentalmente. Como dito na introdução, analisamos um problema específico de três corpos formado por dois férmions (elétrons) e um bóson (bipolaron) na rede cristalina de um dado material. De fatos experimentais e por simplicidade são consideradas algumas suposições no modelo ${ }^{1}$ :

- O spin total do bipolaron é zero.

- O transporte de carga ocorre em uma rede bidimensional, isto devido a estrutura molecular dos cupratos supercondutores, onde o material é constituído de camadas que podem ser consideradas independentes.

- Em contraste com trabalhos precedentes, assumiremos num primeiro momento que o par eletrônico experimenta uma repulsão completamente genérica.

- Com base num princípio de isotropia espacial, todo tipo de interação que há entre as partículas é invariante por rotações de $90^{\circ}$.

- De fatos experimentais tem sido proposto que nos supercondutores com alta $T_{c}$, a interação atrativa entre dois elétrons é mediada por um bipolaron, isto é, um par espacial de elétrons interagindo por meio de fônons ou mágnons. Em vista disso, um bipolaron pode decair em dois elétrons, que por sua vez podem fundir-se num bipolaron.

\footnotetext{
${ }^{1}$ Para mais detalhes, veja os artigos originais.
} 
- Dado que a massa do bipolaron é muito maior que a dos elétrons, por conservação do momento linear sua energia cinética é pequena quando comparada a dos elétrons.

\subsection{Hamiltoniano do Tipo Hubbard com Termo de Interação Bipolaron-Elétron}

"Nenhuma grande descoberta foi feita jamais sem um palpite ousado."

Isaac Newton ${ }^{2}$

Levando em conta tudo o que foi previamente discutido, o espaço de Hilbert para um elétron é representado por

$$
\ell^{2}\left(\mathbb{Z}^{2} \times\{\uparrow, \downarrow\}\right)
$$

e o correspondente para um bipolaron (com spin zero) será

$$
\ell^{2}\left(\mathbb{Z}^{2}\right)
$$

Por consequência, o espaço de Hilbert associado ao sistema composto ora por dois elétrons ora por um bipolaron corresponde a soma direta

$$
\begin{aligned}
\mathbb{H} & \doteq \operatorname{Alt}^{2}\left(\ell^{2}\left(\mathbb{Z}^{2} \times\{\uparrow, \downarrow\}\right)\right) \oplus \operatorname{Sym}^{1}\left(\ell^{2}\left(\mathbb{Z}^{2}\right)\right) \\
& =\operatorname{Alt}^{2}\left(\ell^{2}\left(\mathbb{Z}^{2} \times\{\uparrow, \downarrow\}\right)\right) \oplus \ell^{2}\left(\mathbb{Z}^{2}\right)
\end{aligned}
$$

Com base na interpretação probabilística da mecânica quântica, inferimos também que um bipolaron cuja função de onda normalizada é

$$
\delta_{x} \in \ell^{2}\left(\mathbb{Z}^{2}\right)=\mathrm{L}^{2}\left(\mathbb{Z}^{2}, \mathbb{P}\left(\mathbb{Z}^{2}\right), \mathfrak{c}\right)
$$

encontra-se na posição $x \in \mathbb{Z}^{2}$ da rede. De fato, $\left|\delta_{x}\right|^{2}$ é visto pela interpretação de Born como a densidade de probabilidade relativo à ocupação espacial, e daí

$$
\mathbb{P}[x]=\int_{\{x\}}\left|\delta_{x}(z)\right|^{2} \mathfrak{c}(\mathrm{d} z)=\int_{\mathbb{Z}^{2}} \chi_{\{x\}}(z) \mathfrak{c}(\mathrm{d} z)=\mathfrak{c}(\{x\})=1
$$

Embora o princípio de incerteza de Heisenberg estabeleça a impossibilidade de se localizar uma partícula com precisão absoluta, lembre-se de que os pontos da rede representam células elementares de um determinado cristal, e que obviamente possui alguma extensão espacial.

Similarmente, um elétron cuja função de onda normalizada é $\delta_{x, s}$ diz-se estar na posição $x$ com spin $s$. Compreendido a pré disposição do sistema, encurtaremos (por questões estéticas) a notação dos seguintes operadores:

$$
b_{x}=b_{1}\left(\delta_{x}\right) \quad, \quad b_{x}^{\dagger}=b_{0}^{\dagger}\left(\delta_{x}\right) \quad, \quad n_{x, s}=n_{2}^{-}\left(\delta_{(x, s)}\right) \quad, \quad a_{x, s}=a_{i}\left(\delta_{(x, s)}\right) \quad, \quad a_{x, s}^{\dagger}=a_{j}^{\dagger}\left(\delta_{(x, s)}\right)
$$

onde $i \in\{1,2\}$ e $j \in\{0,1\}$. Assumimos o risco de denotar operadores diferentes pelo mesmo símbolo na crença de que o leitor irá facilmente distinguí-los conforme o contexto.

Com isso propomos um Hamiltoniano (de volume infinito) cuja parte fermiônica é descrita pelo operador

\footnotetext{
${ }^{2}$ Isaac Newton, $\odot(04 / 01 / 1643)-\dagger(31 / 03 / 1727)$
} 


$$
H_{f}:\left\langle\wedge^{2}\left\{\delta_{(x, s)}: s \in\{\uparrow, \downarrow\}, x \in \mathbb{Z}^{2}\right\}\right\rangle \longrightarrow \operatorname{Alt}^{2}\left(\ell^{2}\left(\mathbb{Z}^{2} \times\{\uparrow, \downarrow\}\right)\right)
$$

definido pontualmente conforme a expressão

$$
H_{f} \doteq \epsilon\left(-\frac{1}{2} \sum_{\substack{x, y \in \mathbb{Z}^{2}:|x-y|=1 \\ s \in\{\uparrow, \downarrow\}}} a_{x, s}^{\dagger} a_{y, s}+2 \sum_{x \in \mathbb{Z}^{2}, s \in\{\uparrow, \downarrow\}} a_{x, s}^{\dagger} a_{x, s}\right)+\sum_{x, z \in \mathbb{Z}^{2}} \mathrm{U}(z) n_{x, \uparrow} n_{x+z, \downarrow}
$$

O parâmetro $\epsilon \geq 0$ quantifica a amplitude de hopping e

$$
\mathrm{U}: \mathbb{Z}^{2} \longrightarrow[0,+\infty)
$$

é uma função $\mathbb{Z}^{2}$-somável e invariante por rotações de $90^{\circ}$ que representa a repulsão eletrônica. Do ponto de vista físico, estamos sendo bastante generosos ao assumir $\mathrm{U} \in \mathrm{L}^{1}\left(\mathbb{Z}^{2}\right)$, visto que por razões empíricas o potencial de repulsão em cristais é extremamente localizado e decai (pelo menos em teoria) exponencialmente devido ao efeito de screening.

Para $(p, t),(q, r) \in \mathbb{Z}^{2} \times\{\uparrow, \downarrow\}$ distintos, note que a expressão

$$
a_{y, s}\left(\delta_{(p, t)} \wedge \delta_{(q, r)}\right)=\frac{1}{\sqrt{2}}\left\{\left\langle\delta_{(y, s)}, \delta_{(p, t)}\right\rangle_{2} \delta_{(q, r)}-\left\langle\delta_{(y, s)}, \delta_{(q, r)}\right\rangle_{2} \delta_{(p, t)}\right\}
$$

se anula sempre que o par $(y, s)$ for diferente tanto de $(p, t)$ quanto de $(q, r)$, e daí

$$
\begin{aligned}
\sum_{\substack{x, y \in \mathbb{Z}^{2}:|x-y|=1 \\
s \in\{\uparrow, \downarrow\}}} a_{x, s}^{\dagger} a_{y, s}\left(\delta_{(p, t)} \wedge \delta_{(q, r)}\right) & =\sum_{x \in \mathbb{Z}^{2}:|x-p|=1} a_{x, t}^{\dagger} a_{p, t}\left(\delta_{(p, t)} \wedge \delta_{(q, r)}\right)+\sum_{x \in \mathbb{Z}^{2}:|x-q|=1} a_{x, r}^{\dagger} a_{q, r}\left(\delta_{(p, t)} \wedge \delta_{(q, r)}\right) \\
& =\frac{1}{\sqrt{2}} \sum_{x \in \mathbb{Z}^{2}:|x-p|=1} a_{x, t}^{\dagger}\left(\delta_{(q, r)}\right)-\frac{1}{\sqrt{2}} \sum_{x \in \mathbb{Z}^{2}:|x-q|=1} a_{x, r}^{\dagger}\left(\delta_{(p, t)}\right) \\
& =\sum_{x \in \mathbb{Z}^{2}:|x-p|=1} \delta_{(x, t)} \wedge \delta_{(q, r)}+\sum_{x \in \mathbb{Z}^{2}:|x-q|=1} \delta_{(p, t)} \wedge \delta_{(x, r)}
\end{aligned}
$$

Da somatória subsequente provém mais dois termos

$$
\begin{aligned}
\sum_{x \in \mathbb{Z}^{2}, s \in\{\uparrow, \downarrow\}} n_{x, s}\left(\delta_{(p, t)} \wedge \delta_{(q, r)}\right) & =n_{p, t}\left(\delta_{(p, t)} \wedge \delta_{(q, r)}\right)+n_{q, r}\left(\delta_{(p, t)} \wedge \delta_{(q, r)}\right) \\
& =\delta_{(p, t)} \wedge \delta_{(q, r)}+\delta_{(p, t)} \wedge \delta_{(q, r)}
\end{aligned}
$$

e com isso vemos que parte cinética de $H_{f}$ (ou seja, tudo o que está entre parênteses) atua em $\delta_{(p, t)} \wedge \delta_{(q, r)}$ superpondo mais oito funções (de onda), cada um delas dizendo respeito ao sistema quando um dos elétrons encontra-se deslocado para uma das células adjacentes à sua posição inicial. Note que isto implementa o movimento de difusão das partículas na rede. Por fim, a ultima somatória se anula completamente quando os spins dos elétrons apontam no mesmo sentido, e do contrário

$$
\begin{aligned}
\sum_{x, z \in \mathbb{Z}^{2}} \mathrm{U}(z) n_{x, \uparrow} n_{x+z, \downarrow}\left(\delta_{(p, \downarrow)} \wedge \delta_{(q, \uparrow)}\right) & =\mathrm{U}(p-q) n_{q, \uparrow} n_{q+(p-q), \downarrow}\left(\delta_{(p, \downarrow)} \wedge \delta_{(q, \uparrow)}\right) \\
& =\mathrm{U}(p-q) n_{q, \uparrow} n_{p, \downarrow}\left(\delta_{(p, \downarrow)} \wedge \delta_{(q, \uparrow)}\right) \\
& =\mathrm{U}(p-q) \delta_{(p, \downarrow)} \wedge \delta_{(q, \uparrow)}
\end{aligned}
$$


ou ainda

$$
\begin{aligned}
\sum_{x, z \in \mathbb{Z}^{2}} \mathrm{U}(z) n_{x, \uparrow} n_{x+z, \downarrow}\left(\delta_{(p, \uparrow)} \wedge \delta_{(q, \downarrow)}\right) & =\mathrm{U}(q-p) n_{p, \uparrow} n_{p+(q-p), \downarrow}\left(\delta_{(p, \uparrow)} \wedge \delta_{(q, \downarrow)}\right) \\
& =\mathrm{U}(p-q) n_{p, \uparrow} n_{q, \downarrow}\left(\delta_{(p, \uparrow)} \wedge \delta_{(q, \downarrow)}\right) \\
& =\mathrm{U}(p-q) \delta_{(p, \uparrow)} \wedge \delta_{(q, \downarrow)}
\end{aligned}
$$

uma vez que $p-q$ e $q-p$ encontram-se defasados por uma rotação de $180^{\circ}$, tornando-se idênticos relativamente a U. Em síntese,

$$
\begin{array}{r}
H_{f}\left(\delta_{(p, t)} \wedge \delta_{(q, r)}\right)=-\frac{\epsilon}{2}\left(\sum_{z \in \mathbb{Z}^{2}:|z|=1} \delta_{(p+z, t)} \wedge \delta_{(q, r)}+\sum_{z \in \mathbb{Z}^{2}:|z|=1} \delta_{(p, t)} \wedge \delta_{(q+z, r)}\right)+ \\
+\left\{\left(1-\delta_{t, r}\right) \mathrm{U}(p-q)+4 \epsilon\right\} \delta_{(p, t)} \wedge \delta_{(q, r)}
\end{array}
$$

sempre que $(p, t)$ e $(q, r) \in \mathbb{Z}^{2} \times\{\uparrow, \downarrow\}$ forem distintos. Elucidado a boa definição de $H_{f}$, a parte bosônica do Hamiltoniano não passa de uma mera reformulação do termo cinético de $H_{f}$ para bósons, sendo ele

$$
H_{b}:\left\langle\delta_{x}: x \in \mathbb{Z}^{2}\right\rangle \longrightarrow \ell^{2}\left(\mathbb{Z}^{2}\right)
$$

definido pontualmente conforme a regra

$$
H_{b} \doteq \epsilon\left(-\frac{h_{b}}{2} \sum_{x, y \in \mathbb{Z}^{2}:|x-y|=1} b_{x}^{\dagger} b_{y}+2 h_{b} \sum_{x \in \mathbb{Z}^{2}} b_{x}^{\dagger} b_{x}\right)
$$

em que $h_{b} \geq 0$ quantifica a energia do bipolaron relativa a dos elétrons. Não há objeções quanto a limitação de $H_{b}$, afinal de contas

$$
H_{b}\left(\delta_{p}\right)=\epsilon h_{b}\left(2 \delta_{p}-\frac{1}{2} \sum_{z \in \mathbb{Z}^{2}:|z|=1} \delta_{p+z}\right), \quad p \in \mathbb{Z}^{2}
$$

sendo que $\delta_{p} \longmapsto \delta_{p+z}$ é unitário. O termo que codifica o decaimento do bipolaron em dois elétrons é descrito pelo operador

$$
W_{b \rightarrow f}:\left\langle\delta_{x}: x \in \mathbb{Z}^{2}\right\rangle \longrightarrow \operatorname{Alt}^{2}\left(\ell^{2}\left(\mathbb{Z}^{2} \times\{\uparrow, \downarrow\}\right)\right)
$$

pontualmente definido pela expressão

$$
W_{b \rightarrow f} \doteq \frac{1}{\sqrt{2}} \sum_{x, y \in \mathbb{Z}^{2}} v(x-y) c_{y}^{\dagger} b_{x}
$$

Como foi dito nas suposições originais, também deve-se assumir que a função de acoplamento $v$ é real, $\mathbb{Z}^{2}$-somável e invariante por rotações de $90^{\circ}$. Em símbolos,

$$
v(x, y)=v(-y, x), \quad x, y \in \mathbb{Z}
$$


O próximo resultado mostra que a transformada de Fourier discreta $\hat{v}$ conserva estas simetrias.

Proposição 60. São verdadeiras as seguintes afirmações:

a) $\hat{v}$ é invariante por rotações de $90^{\circ}$, isto é, $\hat{v}(h, k)=\hat{v}(-k, h)$ para todo $h, k \in \mathbb{R}$.

b) $\hat{v}$ assume apenas valores reais.

Demonstração. As ideias envolvidas nas demonstrações de ambos os itens são praticamente as mesmas, e por isso só provaremos b). Com efeito, $v$ também é invariante por rotações de $180^{\circ}$, e daí

$$
\overline{\hat{v}(k)}=\int_{\mathbb{Z}^{2}} e^{\imath\langle k, x\rangle} v(x) \mathfrak{c}(\mathrm{d} x)=\int_{\mathbb{Z}^{2}} e^{-\imath\langle k,-x\rangle} v(-x) \mathfrak{c}(\mathrm{d} x) \stackrel{3}{=} \int_{\mathbb{Z}^{2}} e^{-\imath\langle k, x\rangle} v(x) \mathfrak{c}(\mathrm{d} x)=\hat{v}(k)
$$

completando assim a demonstração.

Na definição de $W_{b \rightarrow f}$, resta dizer que

$$
c_{y}^{\dagger} \doteq \sum_{z \in \mathbb{Z}^{2},|z| \leq 1}\left(a_{y+z, \uparrow}^{\dagger} a_{y, \downarrow}^{\dagger}+a_{y+z, \uparrow}^{\dagger} a_{y-z, \downarrow}^{\dagger}\right), \quad y \in \mathbb{Z}^{2}
$$

Sua atuação pode ser vista como a criação de um par eletrônico (com spins trocados) nas adjacências do ponto $y$. Com isso, perceba que a função de $v$ na definição de $W_{b \rightarrow f}$ é estabelecer pesos de modo que a chance de se criar um par eletrônico na vizinhança do ponto $y$ diminui a medida que este se afasta do ponto $x$ onde o bipolaron foi destruído. Para todo $p \in \mathbb{Z}^{2}$, observe que a série

$$
\begin{aligned}
\frac{1}{\sqrt{2}} \sum_{x, y \in \mathbb{Z}^{2}} v(x-y) c_{y}^{\dagger} b_{x}\left(\delta_{p}\right)=\frac{1}{\sqrt{2}} \sum_{x, y \in \mathbb{Z}^{2}} v(x-y) c_{y}^{\dagger}\left(\delta_{x, p}\right)=\frac{1}{\sqrt{2}} \sum_{y \in \mathbb{Z}^{2}} v(p-y) c_{y}^{\dagger}(1)= \\
=\sum_{y \in \mathbb{Z}^{2}} v(p-y) \sum_{z \in \mathbb{Z}^{2}:|z| \leq 1}\left(\delta_{(y+z, \uparrow)} \wedge \delta_{(y, \downarrow)}+\delta_{(y+z, \uparrow)} \wedge \delta_{(y-z, \downarrow)}\right)
\end{aligned}
$$

é absolutamente (e portanto simplesmente) convergente, uma vez que

$$
\frac{1}{\sqrt{2}} \sum_{x, y \in \mathbb{Z}^{2}}\left\|v(x-y) c_{y}^{\dagger} b_{x}\left(\delta_{p}\right)\right\|_{\wedge} \leq 10 \sum_{y \in \mathbb{Z}^{2}}|v(p-y)| \stackrel{4}{=} 10 \cdot\|v\|_{1}<+\infty
$$

Por fim, o processo de combinação de dois elétrons para formar um bipolaron é implementado pelo operador

$$
W_{f \rightarrow b} \doteq W_{b \rightarrow f}^{*}
$$

Finalmente, o Hamiltoniano total do sistema é definido via a seguinte matriz de operadores:

$$
H \doteq\left(\begin{array}{cc}
H_{f} & W_{b \rightarrow f} \\
W_{f \rightarrow b} & H_{b}
\end{array}\right) \stackrel{5}{\in} \mathcal{B}(\mathbb{H})
$$

${ }^{3} \mathbb{Z}^{2} \ni x \longmapsto-x \in \mathbb{Z}^{2}$ é inversa de sí mesma, e portanto bijetora.

${ }^{4} y \longrightarrow p-y$ também é inversa de sí mesma. 
Ao contrário do que se aprende nos cursos elementares de mecânica quântica onde os observáveis são dados por operadores ilimitados, podemos dizer que a natureza limitada do Hamiltoniano acima provém, em última análise, do aspecto discreto do modelo: O operador momento (por exemplo) em sua representação usual no contínuo é ilimitado e obtido por derivação. Embora esta operação não seja concebível em um espaço discreto, o que melhor o representa neste caso são operadores de diferença (que são limitados), ou seja, tais quantidades físicas que no contínuo são usualmente ilimitadas tornam-se limitadas no caso discreto. Existem vários outros fenômenos exóticos e contraintuitivos que ocorrem em sistemas discretos como o de massa negativa que discutimos mais à frente.

Definição 61 (Subespaço de Spin Zero). Denominamos

$$
\mathfrak{s p i n}_{0}(\mathbb{H}) \doteq \overline{\left\langle\delta_{(x, \uparrow)} \wedge \delta_{(y, \downarrow)}: x, y \in \mathbb{Z}^{2}\right\rangle} \oplus \ell^{2}\left(\mathbb{Z}^{2}\right)
$$

como sendo o subespaço de spin zero de $\mathbb{H}$.

Como assumimos que o bipolaron tem spin total zero, por conservação do momento do angular os elétrons resultantes devem ter spins trocados, de forma que todos os estados acessíveis ao sistema físico estejam incorporados no espaço de spin zero. Em virtude da seguinte proposição, passaremos então a estudar

$$
\hat{H} \doteq H\left\lceil\mathfrak{s p i n}_{0}(\mathbb{H})\right.
$$

na próxima seção.

Proposição 62. $\mathfrak{s p i n}_{0}(\mathbb{H})$ é um subespaço $H$-invariante de $\mathbb{H}$.

Demonstração. Nitidamente, pois

$$
W_{b \rightarrow f}\left(\delta_{x}\right) \in \overline{\left\langle\delta_{(x, \uparrow)} \wedge \delta_{(y, \downarrow)}: x, y \in \mathbb{Z}^{2}\right\rangle}
$$

e

$$
H_{f}\left(\delta_{(p, \uparrow)} \wedge \delta_{(q, \downarrow)}\right) \in\left\langle\delta_{(x, \uparrow)} \wedge \delta_{(y, \downarrow)}: x, y \in \mathbb{Z}^{2}\right\rangle
$$

sejam quais forem $p, q, x \in \mathbb{Z}^{2}$.

\subsection{Decomposição em Fibras do Setor 2-Férmion-1-Bóson com Spin Total Zero}

"No xadrez, as vezes é preciso entregar uma peça para vencer. Em matemática, as vezes é preciso entregar todo o jogo $6 . "$

Nossa primeira tarefa é mostrar que o espaço de spin zero é isomorfo a alguma integral direta.

\footnotetext{
${ }^{5}$ Confira o apêndice A.

${ }^{6}$ Frase de autoria desconhecida, referindo-se a demonstrações por absurdo em matemática.
} 
Teorema 63. Ponha $E_{\kappa}(x) \doteq e^{\imath\langle x, \kappa\rangle}$. O conjunto $\left\{E_{\kappa}: \kappa \in \mathbb{Z}^{n}\right\}$ forma uma base ortonormal de $\mathrm{L}^{2}\left(\mathbb{T}^{n}, \mathscr{L}\left(\mathbb{T}^{n}\right), \varrho\right)$, em cujo caso

$$
\varrho \doteq \frac{1}{(2 \pi)^{n}}\left(\otimes_{1}^{n} \mathfrak{m}\right)
$$

Demonstração. Dado que $\varrho$ é finita e cada $E_{\kappa}$ contínuo e com valor absoluto identicamente 1 ,

$$
E_{\kappa} \in \mathrm{L}^{2}\left(\mathbb{T}^{n}\right) \subseteq \mathrm{L}^{1}\left(\mathbb{T}^{n}\right) \quad \forall \kappa \in \mathbb{Z}^{n}
$$

o que por um lado assegura a legitimidade de $\left\{E_{\kappa}\right\}_{\kappa \in \mathbb{Z}^{n}}$ como um subconjunto de $\mathrm{L}^{2}\left(\mathbb{T}^{n}\right)$, e por outro que o Teorema de Fubini (adaptado para medidas completas) está liberado nos seguintes cálculos:

$$
\begin{aligned}
\int_{\mathbb{T}^{n}} E_{\kappa} \mathrm{d} \varrho & =\frac{1}{(2 \pi)^{n}} \int_{\mathbb{T}^{n}} E_{\kappa}(x) \mathfrak{m}\left(\mathrm{d}^{n} x\right) \\
& =\frac{1}{(2 \pi)^{n}} \int_{[-\pi, \pi]} \cdots \int_{[-\pi, \pi]} e^{\imath x_{1} \kappa_{1}} \cdots e^{\imath x_{n} \kappa_{n}} \mathrm{~d} \mathfrak{m}\left(x_{1}\right) \cdots \mathrm{d} \mathfrak{m}\left(x_{n}\right) \\
& =\frac{1}{(2 \pi)^{n}}\left(\int_{[-\pi, \pi]} e^{\imath x_{1} \kappa_{1}} \mathrm{~d} \mathfrak{m}\left(x_{1}\right)\right) \cdots\left(\int_{[-\pi, \pi]} e^{\imath x_{n} \kappa_{n}} \mathrm{~d} \mathfrak{m}\left(x_{n}\right)\right) \\
& =\frac{1}{(2 \pi)^{n}} \prod_{i=1}^{n} \int_{-\pi}^{\pi} e^{\imath t \kappa_{i}} \mathrm{~d} t=\delta_{\kappa_{1}, 0} \cdots \delta_{\kappa_{n}, 0}=\delta_{\kappa, 0}
\end{aligned}
$$

Por consequência,

$$
\left\langle E_{\kappa}, E_{\lambda}\right\rangle_{2}=\int_{\mathbb{T}^{n}} \overline{E_{\kappa}} E_{\lambda} \mathrm{d} \varrho=\int_{\mathbb{T}^{n}} E_{\lambda-\kappa} \mathrm{d} \varrho=\delta_{\kappa-\lambda, 0}=\delta_{\kappa, \lambda} \quad \forall \kappa, \lambda \in \mathbb{Z}^{n}
$$

e a ortonormalidade dos $E_{\kappa}$ está comprovada. Para o passo seguinte, recorde que os polinômios trigonométricos formam um conjunto denso em $\mathrm{C}\left(\mathbb{T}^{n}\right)$ relativamente a norma da convergência uniforme ${ }^{7}$. Mas por

$$
\|f\|_{2}^{2}=\int_{\mathbb{T}^{n}}|f|^{2} \mathrm{~d} \varrho \leq \varrho\left(\mathbb{T}^{n}\right) \cdot \max _{\mathbb{T}^{n}}|f|^{2}=\|f\|_{\infty}^{2} \quad \forall f \in \mathrm{C}\left(\mathbb{T}^{n}\right)
$$

vemos que $\|\cdot\|_{2}$ é mais fina que $\|\cdot\|_{\infty}$ em $C\left(\mathbb{T}^{n}\right)$, e por isso $\left\langle E_{\kappa}: \kappa \in \mathbb{Z}^{n}\right\rangle$ também é denso em $C\left(\mathbb{T}^{n}\right)$ na ótica da norma $\|\cdot\|_{2}$. Por fim, sabemos que

$$
\mathrm{C}_{c}\left(\mathbb{T}^{n}\right)=\mathrm{C}\left(\mathbb{T}^{n}\right)
$$

é denso (particularmente) em $\mathrm{L}^{2}\left(\mathbb{T}^{n}\right)$ pois $\otimes_{1}^{n} \mathfrak{m}$ é de Radon.

Teorema 64. São verdadeiras as seguintes equivalências de espaços de Hilbert:
a) $\ell^{2}\left(\mathbb{Z}^{2}\right) \simeq \mathrm{L}^{2}\left(\mathbb{T}^{2}, \mathscr{L}\left(\mathbb{T}^{2}\right), \varrho\right)$
b) $\ell^{2}\left(\mathbb{Z}^{2} \times \mathbb{Z}^{2}\right) \simeq \int_{\mathbb{T}^{2}}^{\oplus} \mathrm{L}^{2}\left(\mathbb{T}^{2}, \mathscr{L}\left(\mathbb{T}^{2}\right), \varrho\right) \mathrm{d} \varrho$

Demonstração. Vamos mostrar que os operadores unitários

\footnotetext{
${ }^{7}$ Isto é consequência do Teorema de Fejér e não de Stone-Weierstrass, dado que os $E_{\kappa}$ não separa os pontos de $\mathbb{T}^{n}$.
} 


$$
S_{b}[\varphi](x) \doteq \int_{\mathbb{T}^{2}} e^{\imath\langle k, x\rangle} \varphi(k) \mathrm{d} \varrho(k), \quad \varphi \in \mathrm{L}^{2}\left(\mathbb{T}^{2}\right)
$$

$\mathrm{e}$

$$
S_{f}[\Lambda](x, y) \doteq \int_{\mathbb{T}^{2}} e^{\imath\langle k, x\rangle}\left\{\int_{\mathbb{T}^{2}} e^{\imath\langle h, y-x\rangle} \Lambda[k](h) \mathrm{d} \varrho(h)\right\} \mathrm{d} \varrho(k), \quad \Lambda \in \int_{\mathbb{T}^{2}}^{\oplus} \mathrm{L}^{2}\left(\mathbb{T}^{2}\right) \mathrm{d} \varrho
$$

implementam as equivalências estipuladas no enunciado. Com efeito, a imagem de todo $x \in \mathbb{Z}^{2}$ por $S_{b}[\varphi]$ é bem definida pois trata-se do produto interno

$$
S_{b}[\varphi](x)=\left\langle E_{-x}, \varphi\right\rangle_{2}
$$

Pela identidade de Parseval,

$$
\sum_{x \in \mathbb{Z}^{2}}\left|S_{b}[\varphi](x)\right|^{2}=\sum_{x \in \mathbb{Z}^{2}}\left|\left\langle E_{x}, \varphi\right\rangle_{2}\right|^{2}=\|\varphi\|_{2}^{2}<+\infty
$$

e com isso

$$
S_{b}[\varphi] \in \ell^{2}\left(\mathbb{Z}^{2}\right)
$$

Bem definida, $S_{b}$ é linear por conta da linearidade à direita do produto interno, isométrica pelo o que já computamos, e finalmente, sobrejetora, pois

$$
\delta_{p}(x)=\delta_{p, x}=\delta_{-p,-x}=\left\langle E_{-x}, E_{-p}\right\rangle_{2}=S_{b}\left[E_{-p}\right](x) \quad \forall x, p \in \mathbb{Z}^{2} .
$$

Agora, vamos mostrar que $S_{f}$ é unitário. Primeiramente, $S_{f}[\Lambda](x, y)$ encontra-se bem definido pois

$$
\int_{\mathbb{T}^{2}}\left|e^{\imath\langle k, x\rangle}\left\langle E_{x-y}, \Lambda(k)\right\rangle_{2}\right|^{2} \mathrm{~d} \varrho(k) \leq \int_{\mathbb{T}^{2}}\|\Lambda(k)\|_{2}^{2} \mathrm{~d} \varrho(k)=\|\Lambda\|_{2, L^{2}\left(\mathbb{T}^{2}\right)}^{2}<+\infty
$$

e além disso $\mathrm{L}^{2}\left(\mathbb{T}^{2}\right) \subseteq \mathrm{L}^{1}\left(\mathbb{T}^{2}\right)$, em razão da finitude de $\varrho$. Pondo

$$
g_{z}(k) \doteq\left\langle E_{-z}, \Lambda(k)\right\rangle_{2}, \quad k \in \mathbb{T}^{2}, z \in \mathbb{Z}^{2}
$$

e aplicando Tonelli na medida de contagem $\mathfrak{c}$, deduzimos

$$
\begin{aligned}
\sum_{x, y \in \mathbb{Z}^{2}}\left|S_{f}(\Lambda)(x, y)\right|^{2}=\sum_{x, y \in \mathbb{Z}^{2}}\left|\left\langle E_{-x}, g_{y-x}\right\rangle_{2}\right|^{2} \stackrel{8}{=} \sum_{x, y \in \mathbb{Z}^{2}}\left|\left\langle E_{x}, g_{y}\right\rangle_{2}\right|^{2}=\sum_{y \in \mathbb{Z}^{2}} \sum_{x \in \mathbb{Z}^{2}}\left|\left\langle E_{x}, g_{y}\right\rangle_{2}\right|^{2}=\sum_{y \in \mathbb{Z}^{2}}|| g_{y} \|_{2}^{2}= \\
=\sum_{y \in \mathbb{Z}^{2}} \int_{\mathbb{T}^{2}}\left|g_{y}\right|^{2} \mathrm{~d} \varrho=\int_{\mathbb{T}^{2}} \sum_{y \in \mathbb{Z}^{2}}\left|\left\langle E_{y}, \Lambda(k)\right\rangle_{2}\right|^{2} \mathrm{~d} \varrho(k)=\int_{\mathbb{T}^{2}}\|\Lambda(k)\|_{2}^{2} \mathrm{~d} \varrho(k)=\|\Lambda\|_{2, \mathrm{~L}^{2}\left(\mathbb{T}^{2}\right)}^{2}<+\infty
\end{aligned}
$$

onde a comutatividade da somatória com a integral se dá pelo teorema da convergência monótona. Com isso provamos não só que $S_{f}$ é bem definida, ou seja,

$$
S_{f}[\Lambda] \in \ell^{2}\left(\mathbb{Z}^{2} \times \mathbb{Z}^{2}\right)
$$

mas também que ela conserva normas. Deixamos sua linearidade a cargo do leitor, e por fim

$$
\begin{aligned}
S_{f}\left[e^{-\imath\langle\cdot, p\rangle} E_{p-q}\right](x, y)=\int_{\mathbb{T}^{2}} e^{\imath\langle k, x\rangle}\left\{\int_{\mathbb{T}^{2}} e^{\imath\langle h, y-x\rangle} e^{-\imath\langle k, p\rangle} e^{\imath\langle h, p-q\rangle} \mathrm{d} \varrho(h)\right\} \mathrm{d} \varrho(k) & = \\
= & \left(\int_{\mathbb{T}^{2}} e^{-\imath\langle k, p\rangle} e^{\imath\langle k, x\rangle} \mathrm{d} \varrho(k)\right)\left(\int_{\mathbb{T}^{2}} e^{\imath\langle h, p-q\rangle} e^{\imath\langle h, y-x\rangle} \mathrm{d} \varrho(h)\right)
\end{aligned}
$$

aonde

\footnotetext{
${ }^{8}(x, y) \longmapsto(-x, y-x)$ é uma bijeção em $\mathbb{Z}^{2} \times \mathbb{Z}^{2}$, com inversa $(p, q) \longmapsto(-p, q-p)$.
} 


$$
\left\langle E_{p}, E_{x}\right\rangle_{2}\left\langle E_{q-p}, E_{y-x}\right\rangle_{2}=\delta_{p, x} \delta_{q-p, y-x}=\delta_{p}(x) \delta_{q-p}(y-x)=\delta_{(p, q)}(x, y)
$$

Isso conclui a prova.

Corolário 65. $\mathfrak{s p i n}_{0}(\mathbb{H}) \simeq \int_{\mathbb{T}^{2}}^{\oplus} \mathrm{L}^{2}\left(\mathbb{T}^{2}, \mathscr{L}\left(\mathbb{T}^{2}\right), \varrho\right) \oplus \mathbb{C} \mathrm{d} \varrho$

Demonstração. Por efeito da relação unitária $\delta_{(x, \uparrow)} \wedge \delta_{(y, \downarrow)} \longmapsto \delta_{(x, y)}$, tem-se

$$
\overline{\left\langle\delta_{(x, \uparrow)} \wedge \delta_{(y, \downarrow)}: x, y \in \mathbb{Z}^{2}\right\rangle} \simeq \ell^{2}\left(\mathbb{Z}^{2} \times \mathbb{Z}^{2}\right)
$$

de onde

$$
\begin{aligned}
\mathfrak{s p i n}_{0}(\mathbb{H}) & \simeq \ell^{2}\left(\mathbb{Z}^{2} \times \mathbb{Z}^{2}\right) \oplus \ell^{2}\left(\mathbb{Z}^{2}\right) \\
& \simeq\left(\int_{\mathbb{T}^{2}}^{\oplus} \mathrm{L}^{2}\left(\mathbb{T}^{2}, \mathscr{L}\left(\mathbb{T}^{2}\right), \varrho\right) \mathrm{d} \varrho\right) \oplus\left(\int_{\mathbb{T}^{2}}^{\oplus} \mathbb{C} \mathrm{d} \varrho\right) \\
& \simeq \int_{\mathbb{T}^{2}}^{\oplus} \mathrm{L}^{2}\left(\mathbb{T}^{2}, \mathscr{L}\left(\mathbb{T}^{2}\right), \varrho\right) \oplus \mathbb{C} \mathrm{d} \varrho
\end{aligned}
$$

graças também a (P.33).

Cumprido nosso primeiro objetivo, cabe agora conjugar $\hat{H}$ pela transformação unitária

$$
\begin{aligned}
& U: \quad \operatorname{spin}_{0}(\mathbb{H}) \longrightarrow \ell^{2}\left(\mathbb{Z}^{2} \times \mathbb{Z}^{2}\right) \oplus \ell^{2}\left(\mathbb{Z}^{2}\right) \longrightarrow\left(\int_{\mathbb{T}^{2}}^{\oplus} \mathrm{L}^{2}\left(\mathbb{T}^{2}\right) \mathrm{d} \varrho\right) \oplus\left(\int_{\mathbb{T}^{2}}^{\oplus} \mathbb{C} \mathrm{d} \varrho\right) \longrightarrow \cdots \\
& \left(\delta_{(p, \uparrow)} \wedge \delta_{(q, \downarrow)}, \delta_{z}\right) \longmapsto\left(\delta_{(p, q)}, \delta_{z}\right) \longmapsto\left(e^{-\imath \prec \cdot, p\rangle} E_{p-q}, E_{-z}\right) \\
& \cdots \longrightarrow \int_{\mathbb{T}^{2}}^{\oplus} \mathrm{L}^{2}\left(\mathbb{T}^{2}\right) \oplus \mathbb{C} \mathrm{d} \varrho \\
& \cdots \longmapsto U\left(\delta_{(p, \uparrow)} \wedge \delta_{(q, \downarrow)}, \delta_{z}\right) \quad: \quad \mathbb{T}^{2} \longrightarrow \mathrm{L}^{2}\left(\mathbb{T}^{2}\right) \oplus \mathbb{C} \\
& k \longmapsto\left(e^{-\imath\langle k, p\rangle} E_{p-q}, e^{-\imath\langle k, z\rangle}\right)
\end{aligned}
$$

e sobre isso temos o seguinte resultado: 
Teorema 66 (Bru-Pedra-Pasquale, [BdPdSP15]). A conjugação de $\hat{H}$ por U é decomponível pela integral direta de $\mathrm{L}^{2}\left(\mathbb{T}^{2}\right) \oplus \mathbb{C}$ sobre $\mathbb{T}^{2}$ relativamente a medida $\varrho$. Com precisão:

$$
U \hat{H} U^{*}=\int_{\mathbb{T}^{2}}^{\oplus}\left(\begin{array}{ll}
\Xi_{1,1}(k) & \Xi_{1,2}(k) \\
\Xi_{2,1}(k) & \Xi_{2,2}(k)
\end{array}\right) \mathrm{d} \varrho(k)
$$

onde

$$
\begin{aligned}
& \Xi_{1,1}: \mathbb{T}^{2} \longrightarrow \mathcal{B}\left(\mathrm{L}^{2}\left(\mathbb{T}^{2}\right)\right) \quad ; \quad \Xi_{1,1}(k) \varphi \doteq \mathrm{M}_{\mathfrak{f}(k)} \varphi+\sum_{z \in \mathbb{Z}^{2}} \mathrm{U}(z) \mathrm{P}_{z} \varphi \\
& \Xi_{1,2}: \mathbb{T}^{2} \longrightarrow \mathcal{B}\left(\mathbb{C}, \mathrm{L}^{2}\left(\mathbb{T}^{2}\right)\right) \quad ; \quad \Xi_{1,2}(k) z \doteq \hat{v}(k) z \mathfrak{d}(k) \\
& \Xi_{2,1}: \mathbb{T}^{2} \longrightarrow \mathrm{L}^{2}\left(\mathbb{T}^{2}\right)^{*} \quad ; \quad \Xi_{2,1}(k) \varphi \doteq \hat{v}(k)\langle\mathfrak{d}(k), \varphi\rangle_{2} \\
& \Xi_{2,2}: \mathbb{T}^{2} \longrightarrow \mathcal{B}(\mathbb{C}) \quad ; \quad \Xi_{2,2}(k) z \doteq \mathfrak{b}(k) z
\end{aligned}
$$

sendo que $\mathrm{P}_{z}$ denota o projetor ortogonal de $\mathrm{L}^{2}\left(\mathbb{T}^{2}\right)$ no subespaço fechado

$$
\mathbb{C} e^{-\imath\langle\cdot, z\rangle}, \quad z \in \mathbb{Z}^{2}
$$

$e \mathfrak{d}, \mathfrak{f}: \mathbb{T}^{2} \longrightarrow \mathrm{C}\left(\mathbb{T}^{2}\right)$ e $\mathfrak{b} \in \mathrm{C}\left(\mathbb{T}^{2}\right)$ são definidos por

$$
\begin{gathered}
\mathfrak{b}(k) \doteq \epsilon h_{b}\{2-\cos (k)\} \quad, \quad \mathfrak{f}(k)(h) \doteq \epsilon\{4-\cos (h-k)-\cos (h)\} \\
\mathfrak{d}(k)(h) \doteq 2\{\cos (h-k)+\cos (2 h-k)+1\}
\end{gathered}
$$

com a convenção de que

$$
\cos (k) \equiv \cos \left(k_{1}\right)+\cos \left(k_{2}\right), \quad k=\left(k_{1}, k_{2}\right) \in \mathbb{T}^{2}
$$

Denotando as fibras da integral direta acima com a notação mais curta $\Xi(k)$, segue ainda que

$$
\|\hat{H}\|_{\mathrm{op}}=\max _{k \in \mathbb{T}^{2}}\|\Xi(k)\|_{\mathrm{op}}
$$

Demonstração. Antes de tudo, vamos mostrar que

$$
\Xi \in \mathrm{L}_{\mathfrak{H}}^{\infty}\left(\mathbb{T}^{2}, \mathscr{L}\left(\mathbb{T}^{2}\right), \varrho\right) \quad \mathfrak{H} \doteq \mathrm{L}^{2}\left(\mathbb{T}^{2}\right) \oplus \mathbb{C}
$$

Para tal, é suficiente que $\Xi$ seja contínua, dada a compacidade de $\mathbb{T}^{2}$. Com efeito,

$$
\left\{\mathfrak{f}(\cdot)(h) \in \mathrm{C}\left(\mathbb{T}^{2}\right): h \in \mathbb{T}^{2}\right\}
$$

é um conjunto $\epsilon$-equilipschitz pois o interior do 2-torus é um aberto conexo de $\mathbb{R}^{2}$ (denso em $\mathbb{T}^{2}$ ) onde a diferencial de cada $f_{h}=\mathfrak{f}(\cdot)(h)$ cumpre

$$
\frac{\partial f_{h}}{\partial k_{i}}(k)=-\epsilon \sin \left(h_{i}-k_{i}\right) \Longrightarrow\left\|\mathrm{d} f_{h}(k)\right\|_{\text {op }}=\left|\vec{\nabla} f_{h}(k)\right| \leq \epsilon, \quad k \in \operatorname{int}\left(\mathbb{T}^{2}\right)
$$

Daí resulta a continuidade de $\Xi_{1,1}$, visto que para um dado $\varepsilon>0$ e $k_{0} \in \mathbb{T}^{2}$, a equicontinuidade acima nos 
fornece $\delta>0$ de tal modo que

$$
\begin{array}{r}
k \in \mathbb{T}^{2}:\left|k-k_{0}\right|<\delta \Longrightarrow\left|\mathfrak{f}(k)(h)-\mathfrak{f}\left(k_{0}\right)(h)\right|<\varepsilon \quad \forall h \in \mathbb{T}^{2} \Longrightarrow\left\|\Xi_{1,1}(k)-\Xi_{1,1}\left(k_{0}\right)\right\|_{\text {op }}= \\
\left.=\| \mathrm{M}_{\mathfrak{f}(k)}-\mathrm{M}_{\mathfrak{f}\left(k_{0}\right)}\right)\left\|_{\text {op }}=\right\| \mathfrak{f}(k)-\mathfrak{f}\left(k_{0}\right) \|_{\infty}=\sup _{h \in \mathbb{T}^{2}}\left|\mathfrak{f}(k)(h)-\mathfrak{f}\left(k_{0}\right)(h)\right| \leq \varepsilon
\end{array}
$$

Similarmente, mostra-se que $\mathfrak{d}$, e portanto seu produto pontual com $\hat{v}$, é $\|\cdot\|_{\infty}$-contínuo. Mas então $\hat{v} \cdot \mathfrak{d}$ também é $\|\cdot\|_{2}$-contínua, e se composto com

$$
\mathrm{L}^{2}\left(\mathbb{T}^{2}\right) \ni \varphi \longmapsto \varphi^{*} \in \mathrm{L}^{2}\left(\mathbb{T}^{2}\right)^{*}
$$

que é $\left(\|\cdot\|_{2},\|\cdot\|_{\text {op }}\right)$-contínua, resulta em $\Xi_{2,1}$. A continuidade de $\Xi_{1,2}$ é consequência, uma vez que

$$
\left\langle\Xi_{1,2}(k) z, \varphi\right\rangle_{2}=\bar{z} \cdot \hat{v}(k)\langle\mathfrak{d}(k), \varphi\rangle_{2}=\bar{z} \cdot \Xi_{2,1}(k) \varphi \quad z \in \mathbb{C}, \varphi \in \mathrm{L}^{2}\left(\mathbb{T}^{2}\right)
$$

ou seja, $\Xi_{1,2}(k)=\Xi_{2,1}(k)^{*}$ para todo $k \in \mathbb{T}^{2}$. Por fim, não há o que dizer sobre a continuidade de $\Xi_{2,2}$, e o primeiro objetivo foi concluído. Aliás,

$$
\left\|\int_{\mathbb{T}^{2}}^{\oplus} \Xi(k) \mathrm{d} \varrho(k)\right\|_{\text {op }}=\underset{k \in \mathbb{T}^{2}}{\operatorname{ess} \sup }\|\Xi(k)\|_{\text {op }} \stackrel{9}{=} \max _{k \in \mathbb{T}^{2}}\|\Xi(k)\|_{\text {op }}
$$

Agora, considere

$$
\begin{aligned}
& U_{f}: \overline{\left\langle\delta_{(x, \uparrow)} \wedge \delta_{(y, \downarrow)}: x, y \in \mathbb{Z}^{2}\right\rangle} \longrightarrow \int_{\mathbb{T}^{2}}^{\oplus} \mathrm{L}^{2}\left(\mathbb{T}^{2}\right) \mathrm{d} \varrho \quad U_{b}: \ell^{2}\left(\mathbb{Z}^{2}\right) \longrightarrow \int_{\mathbb{T}^{2}}^{\oplus} \mathbb{C} \mathrm{d} \varrho \\
& \delta_{(p, \uparrow)} \wedge \delta_{(q, \downarrow)} \longmapsto e^{-\imath\langle\cdot p\rangle} E_{p-q} \quad \delta_{z} \longmapsto E_{-z}
\end{aligned}
$$

e calculemos cada entrada da matriz de operadores

$$
\left(\begin{array}{cc}
U_{f} & 0 \\
0 & U_{b}
\end{array}\right)\left(\begin{array}{cc}
H_{f} & W_{b \rightarrow f} \\
W_{f \rightarrow b} & H_{b}
\end{array}\right)\left(\begin{array}{cc}
U_{f}^{*} & 0 \\
0 & U_{b}^{*}
\end{array}\right)=\left(\begin{array}{cc}
U_{f} H_{f} U_{f}^{*} & U_{f} W_{b \rightarrow f} U_{b}^{*} \\
U_{b} W_{f \rightarrow b} U_{f}^{*} & U_{b} H_{b} U_{b}^{*}
\end{array}\right)
$$

Com efeito, para todo $p \in \mathbb{Z}^{2}$, tem-se

$$
\begin{aligned}
U_{b} H_{b} U_{b}^{*}\left(e^{-\imath\langle\cdot, p\rangle}\right)=U_{b} H_{b}\left(\delta_{p}\right)=\epsilon h_{b}\left(2 U_{b}\left(\delta_{p}\right)-\frac{1}{2} \sum_{z \in \mathbb{Z}^{2}:|z|=1} U_{b}\left(\delta_{p+z}\right)\right) & = \\
& =\epsilon h_{b}\left(2 e^{-\imath\langle\cdot, p\rangle}-\frac{1}{2} \sum_{z \in \mathbb{Z}^{2}:|z|=1} e^{-\imath\langle\cdot, p+z\rangle}\right)
\end{aligned}
$$

$\log \mathrm{O}$

$$
U_{b} H_{b} U_{b}^{*}\left[e^{-\imath\langle\cdot, p\rangle}\right](k)=\epsilon h_{b}\left(2 e^{-\imath\langle k, p\rangle}-\frac{1}{2} \sum_{z \in \mathbb{Z}^{2}:|z|=1} e^{-\imath\langle k, p+z\rangle}\right)=\epsilon h_{b}\{2-\cos (k)\} e^{-\imath\langle k, p\rangle}=\mathfrak{b}(k) e^{-\imath\langle k, p\rangle}
$$

\footnotetext{
${ }^{9}$ Confira o apêndice A.
} 
e por efeito do (T.63),

$$
U_{b} H_{b} U_{b}^{*}=\int_{\mathbb{T}^{2}}^{\oplus} \mathfrak{b}(k) \mathrm{d} \varrho(k)=\int_{\mathbb{T}^{2}}^{\oplus} \Xi_{2,2}(k) \mathrm{d} \varrho(k)
$$

Em particular, $\mathfrak{b}$ correponde a energia cinética do bipolaron. Na sequência, temos

$$
\begin{aligned}
U_{f} H_{f} U_{f}^{*}\left(e^{-\imath \prec \cdot p\rangle} E_{p-q}\right)=-\frac{\epsilon}{2} \sum_{z \in \mathbb{Z}^{2}:|z|=1}\left\{U_{f}\left(\delta_{(p+z, \uparrow)} \wedge \delta_{(q, \downarrow)}\right)+U_{f}\left(\delta_{(p, \uparrow)} \wedge\right.\right. & \left.\left.\delta_{(q+z, \downarrow)}\right)\right\}+ \\
& +\{\mathrm{U}(p-q)+4 \epsilon\} U_{f}\left(\delta_{(p, \uparrow)} \wedge \delta_{(q, \downarrow)}\right) \\
=-\frac{\epsilon}{2} \sum_{z \in \mathbb{Z}^{2}:|z|=1}\left\{e^{-\imath\langle\cdot p+z\rangle} E_{(p+z)-q}+e^{-\imath \prec \cdot p\rangle} E_{p-(q+z)}\right\}+ & +\{\mathrm{U}(p-q)+4 \epsilon\} e^{-\imath \prec \cdot p\rangle} E_{p-q}
\end{aligned}
$$

e por consequência

$$
\begin{aligned}
U_{f} H_{f} U_{f}^{*}\left[e^{-\imath\langle\cdot p\rangle} E_{p-q}\right](k)(h) & =-\frac{\epsilon}{2} \sum_{z \in \mathbb{Z}^{2}:|z|=1}\left\{e^{-\imath\langle k, p+z\rangle} e^{\imath\langle h,(p+z)-q\rangle}+e^{-\imath\langle k, p\rangle} e^{\imath\langle h, p-(q+z)\rangle}\right\}+ \\
& +\{\mathrm{U}(p-q)+4 \epsilon\} e^{-\imath\langle k, p\rangle} e^{\langle\langle h, p-q\rangle} \\
& =\epsilon e^{-\imath\langle k, p\rangle} e^{\imath\langle h, p-q\rangle}\left(4-\frac{1}{2} \sum_{z \in \mathbb{Z}^{2}:|z|=1}\left\{e^{\imath\langle h-k, z\rangle}+e^{\imath\langle h, z\rangle}\right\}\right)+ \\
& =\mathfrak{f}(k)(h) e^{-\imath\langle k, p\rangle} e^{-\imath\langle h, q-p\rangle}+\mathrm{U}(p-q) e^{-\imath\langle k, p\rangle} e^{\langle\langle h, p-q\rangle} \\
& =\mathrm{M}_{\mathfrak{f}(k)}\left[e^{-\imath\langle k, p\rangle} E_{p-q}\right](h)+\sum_{z \in \mathbb{Z}^{2}} \mathrm{U}(z) \mathrm{P}_{z}\left[e^{-\imath\langle k, p\rangle} E_{p-q}\right](h)
\end{aligned}
$$

dado que

$$
\sum_{z \in \mathbb{Z}^{2}} \mathrm{U}(z) \mathrm{P}_{z}\left[e^{-\imath\langle k, p\rangle} E_{p-q}\right]=e^{-\imath\langle k, p\rangle} \sum_{z \in \mathbb{Z}^{2}} \mathrm{U}(z) \mathrm{P}_{z}\left[E_{p-q}\right]=e^{-\imath\langle k, p\rangle} \mathrm{U}(q-p) E_{p-q}=e^{-\imath\langle k, p\rangle} \mathrm{U}(p-q) E_{p-q}
$$

Pela arbitrariedade de todas as constantes envolvidas, segue

$$
U_{f} H_{f} U_{f}^{*}=\int_{\mathbb{T}^{2}}^{\oplus}\left(\mathrm{M}_{\mathfrak{f}(k)}+\sum_{z \in \mathbb{Z}^{2}} \mathrm{U}(z) \mathrm{P}_{z}\right) \mathrm{d} \varrho(k)=\int_{\mathbb{T}^{2}}^{\oplus} \Xi_{1,1}(k) \mathrm{d} \varrho(k)
$$

Nunca é tarde para lembrar que

$$
\sum_{\mathbb{Z}^{2}}|| \mathrm{U}(z) \mathrm{P}_{z} \|_{\mathrm{op}}=\sum_{\mathbb{Z}^{2}}|\mathrm{U}(z)|<+\infty
$$

ou seja, $\sum \mathrm{U}(z) \mathrm{P}_{z}$ converge absolutamente, e portanto simplesmente, graças a completude de $\mathcal{B}\left(\mathrm{L}^{2}\left(\mathbb{T}^{2}\right)\right)$. Doravante, 


$$
U_{f} W_{b \rightarrow f} U_{b}^{*}\left[e^{-\imath\langle\cdot, p\rangle}\right]=\sum_{y \in \mathbb{Z}^{2}} v(p-y) \sum_{z \in \mathbb{Z}^{2}:|z| \leq 1} e^{-\imath\langle\cdot, y+z\rangle} E_{(y+z)-y}+e^{-\imath\langle\cdot, y+z\rangle} E_{(y+z)-(y-z)}
$$

de onde se deduz

$$
\begin{aligned}
U_{f} W_{b \rightarrow f} U_{b}^{*}\left[e^{-\imath\langle\cdot, p\rangle}\right](k)(h) & =\sum_{y \in \mathbb{Z}^{2}} v(p-y) \sum_{z \in \mathbb{Z}^{2}:|z| \leq 1} e^{-\imath\langle k, y+z\rangle} e^{\imath\langle h, z\rangle}+e^{-\imath\langle k, y+z\rangle} e^{\imath\langle h, 2 z\rangle} \\
& =\sum_{y \in \mathbb{Z}^{2}} v(y-p) e^{-\imath\langle k, y\rangle} \sum_{z \in \mathbb{Z}^{2}:|z| \leq 1} e^{\imath\langle h-k, z\rangle}+e^{\imath\langle 2 h-k, z\rangle} \\
& =\mathfrak{d}(k)(h) e^{-\imath\langle k, p\rangle} \sum_{y \in \mathbb{Z}^{2}} v(y-p) e^{-\imath\langle k, y-p\rangle} \\
& 10 \\
& =\hat{v}(k) e^{-\imath\langle k, p\rangle} \mathfrak{d}(k)(h) .
\end{aligned}
$$

Como $h, k$ e $p$ foram escolhidos ao acaso, verificamos que $U_{f} W_{b \rightarrow f} U_{b}^{*}$ coincide com

$$
\begin{aligned}
J: \int_{\mathbb{T}^{2}}^{\oplus} \mathbb{C} \mathrm{d} \varrho \longrightarrow \int_{\mathbb{T}^{2}}^{\oplus} \mathrm{L}^{2}\left(\mathbb{T}^{2}\right) \mathrm{d} \varrho \\
\varphi \longmapsto J \varphi: \mathbb{T}^{2} \longrightarrow \mathrm{L}^{2}\left(\mathbb{T}^{2}\right) \\
k \longmapsto \Xi_{1,2}(k) \varphi(k)
\end{aligned}
$$

numa base ortonormal de $\mathrm{L}^{2}\left(\mathbb{T}^{2}\right)$, e logo em toda sua extensão ${ }^{11}$. Para terminar,

$$
\begin{aligned}
\left\langle\left(U_{b} W_{f \rightarrow b} U_{f}^{*}\right) \Psi, \varphi\right\rangle_{2}=\left\langle\Psi,\left(U_{f} W_{b \rightarrow f} U_{b}^{*}\right) \varphi\right\rangle_{2, \mathrm{~L}^{2}\left(\mathbb{T}^{2}\right)} & = \\
& =\int_{\mathbb{T}^{2}}\left\langle\Psi(k), \Xi_{1,2}(k) \varphi(k)\right\rangle_{2} \mathrm{~d} \varrho(k)=\int_{\mathbb{T}^{2}} \overline{\Xi_{2,1}(k) \Psi(k)} \varphi(k) \mathrm{d} \varrho(k)
\end{aligned}
$$

mostra que

$$
\left(U_{b} W_{f \rightarrow b} U_{f}^{*}\right) \Psi(k)=\Xi_{2,1}(k) \Psi(k)
$$

sejam quais forem $k \in \mathbb{T}^{2}$ e $\Psi$ no espaço de $L^{2}\left(\mathbb{T}^{2}\right)$-fibras.

Cabe observar que toda essa abordagem permanece válida em qualquer dimensão de modo que se consideramos $\mathbb{Z}^{n}$ no lugar de $\mathbb{Z}^{2}$, basta trocar $\mathbb{T}^{2}$ por $\mathbb{T}^{n}$ com a convenção de que

$$
\cos (k) \equiv \cos \left(k_{1}\right)+\cdots+\cos \left(k_{n}\right), \quad k=\left(k_{1}, \ldots, k_{n}\right) \in \mathbb{T}^{n}
$$

É claro que dimensões mais elevadas dificultam uma possível análise numérica, mas como observamos no início do capítulo a dimensão que é considerada em física é $d=2$ porque os materiais onde a supercondutividade em alta temperatura ocorre são estratificados, ou seja, a condução elétrica acontece em camadas.

\footnotetext{
${ }^{10} y \longmapsto y-p$ é uma bijeção em $\mathbb{Z}^{2}$, com inversa $x \longmapsto x+p$.

${ }^{11}$ Para não prolongar demais esta demonstração, omitimos a verificação de que tal aplicação define um mapa linear limitado entre estes dois espaços.
} 
Por fim, note que o princípio físico que está por trás da decomposição em fibras acima é a invariância por translação: Em mecânica clássica, um sistema de $n$ partículas cujas leis dinâmicas são invariantes por translações temporais pode ser reduzido a um sistema de $n-1$ partículas indo para o seu centro de massa, e basicamente é este o mecanismo que possibilita a decomposição; trata-se de uma versão de redução ao centro de massa escrita na rede, possível graças a uma simetria presente no sistema.

\subsection{Espectro das Fibras de $\hat{H}$}

"Nunca ande por trilhas, pois assim só irá até onde outros já foram."

\section{Graham Bell}

A parte essencial do espectro de cada fibra de $\hat{H}$ está completamente determinada pela proposição abaixo:

Proposição 67 (Bru-Pedra-Pasquale, [BdPdSP15]). Para cada $k \in \mathbb{T}^{2}$ que fixamos, são verdadeiras as seguintes relações:

a) $\sigma_{\text {ess }}(\Xi(k))=\sigma_{\text {ess }}\left(\Xi_{1,1}(k)\right)=\operatorname{Im}(\mathfrak{f}(k))=2 \epsilon \cos (k / 2)[-1,1]+4 \epsilon$

b) $\min \sigma\left(\Xi_{1,1}(k)\right)=4 \epsilon-2 \epsilon \cos (k / 2)$

Demonstração. Se $\lambda$ é um autovalor de $\mathrm{M}_{\mathfrak{f}(k)}$ e $\varphi \in \mathrm{L}^{2}\left(\mathbb{T}^{2}\right)$ um autovetor corresponde, tem-se

$$
\mathfrak{f}(k)(h) \varphi(h)=\lambda \varphi(h) \quad \sigma \text {-q.t.p }
$$

Mas como $\varphi \neq 0$, existe $S \in \mathscr{L}\left(\mathbb{T}^{2}\right)$ com medida (de Lebesgue) estritamente positiva onde $\varphi$ não se anula, e por isso

$$
\mathfrak{f}(k)(h)=\lambda \quad \forall h \in S .
$$

Dado que a fronteira de $\mathbb{T}^{2}$ tem medida nula, não há problema em supor que $S$ está contido em seu interior. Oras, se

$$
\mathfrak{f}(k)-\lambda \in \mathrm{C}^{\omega}\left(\operatorname{int}\left(\mathbb{T}^{2}\right)\right)
$$

e os zeros de funções reais analíticas formam um conjunto de medida (de Lebesgue) nula, com maior razão $\varrho(S)=0$, sendo que isso é um absurdo. O erro foi supor que $\mathrm{M}_{\mathfrak{f}(k)}$ possui autovalor, e daí

$$
\sigma_{\text {ess }}\left(\mathrm{M}_{\mathfrak{f}(k)}\right)=\sigma\left(\mathrm{M}_{\mathfrak{f}(k)}\right)=\operatorname{ess} \operatorname{im}(\mathfrak{f}(k)) \stackrel{12}{=} \operatorname{Im}(\mathfrak{f}(k))
$$

onde não se ausentamos em mencionar o (C.41). Mas agora, em conformidade com a compacidade de $\Xi_{2,1}(k), \Xi_{1,2}(k)$ e todo $\mathrm{P}_{z}^{13}$, aplica-se (T.24), que junto de (P.21), resulta

$$
\sigma_{\mathrm{ess}}(\Xi(k))=\sigma_{\mathrm{ess}}\left(\mathrm{M}_{\mathfrak{f}(k)} \oplus \Xi_{2,2}(k)\right)=\sigma_{\mathrm{ess}}\left(\mathrm{M}_{\mathfrak{f}(k)}\right)
$$

Como $\mathfrak{f}(k)$ é contínua e $\mathbb{T}^{2}$ um conexo compacto, sua imagem é um intervalo compacto de $\mathbb{R}$, e portanto fica determinado pelos seus extremos. Efetivamente, $\mathfrak{f}(k)$ se escreve como soma independente de duas funções

\footnotetext{
${ }^{12}$ Consulte o apêndice A para mais detalhes.

${ }^{13}$ Claro, pois todos eles possuem posto finito e igual à 1 .
} 


$$
\mathfrak{f}(k)(x, y)=f_{1}(x)+f_{2}(y)
$$

em cujo caso

$$
f_{i}(x) \doteq \epsilon\left\{2-\cos \left(x-k_{i}\right)-\cos (x)\right\}, \quad x \in[-\pi, \pi]
$$

e com isso seu máximo e mínimo resulta da soma dos máximos e mínimos individuais de $f_{1} \operatorname{com} f_{2}$. Oras,

$$
\begin{aligned}
f_{i}(x) & =\epsilon\left\{2-\cos (x) \cos \left(k_{i}\right)-\sin (x) \sin \left(k_{i}\right)-\cos (x)\right\} \\
& =\epsilon\left\{2-\cos (x)\left(\cos \left(k_{i}\right)+1\right)-\sin (x) \sin \left(k_{i}\right)\right\} \\
& =\epsilon\left\{2-2 \cos (x) \cos ^{2}\left(k_{i} / 2\right)-2 \sin (x) \sin \left(k_{i} / 2\right) \cos \left(k_{i} / 2\right)\right\} \\
& =2 \epsilon-2 \epsilon \cos \left(k_{i} / 2\right)\left\{\cos (x) \cos \left(k_{i} / 2\right)+\sin (x) \sin \left(k_{i} / 2\right)\right\} \\
& =2 \epsilon-2 \epsilon \cos \left(k_{i} / 2\right) \cos \left(x-k_{i} / 2\right), \quad x \in[-\pi, \pi]
\end{aligned}
$$

sendo que o termo $\cos \left(x-k_{i} / 2\right)$ atinge seu máximo e mínimo global (no caso \pm 1 ) em $[-\pi, \pi]$ porque este é um intervalo compacto de mesmo comprimento que o período do cosseno. Para o item subsequente, basta aplicar o (C.4) na desigualdade $\mathrm{M}_{\mathfrak{f}(k)} \leq \Xi_{1,1}(k)$.

Para que se tenha

$$
\mathfrak{b}(k) \leq 2 h_{b}\{4 \epsilon-2 \epsilon \cos (k / 2)\}, \quad k \in \mathbb{T}^{2}
$$

a grande maioria dos resultados à frente restringe os valores de $h_{b}$ ao intervalo $\left[0, \frac{1}{2}\right]$. De fato,

$$
\frac{1}{2}\{1-\cos (t)\}=\sin ^{2}\left(\frac{t}{2}\right)=4 \sin ^{2}\left(\frac{t}{4}\right) \cos ^{2}\left(\frac{t}{4}\right) \leq 4 \sin ^{2}\left(\frac{t}{4}\right)=2\left\{1-\cos \left(\frac{t}{2}\right)\right\}, \quad t \in \mathbb{R}
$$

Note também que a igualdade ocorre apenas em $k=0$, pois

$$
\cos ^{2}\left(\frac{t}{4}\right)=1 \Longleftrightarrow \cos \left(\frac{t}{4}\right)= \pm 1 \Longleftrightarrow t=4 n \pi, \quad n \in \mathbb{Z}
$$

sendo $n=0$ o único inteiro para o qual $4 n \pi \in[-\pi, \pi]$.

Vale dizer que não há perda considerável de generalidade ao confinar os valores de $h_{b}$ nesse intervalo pois já é de se esperar que seu valor experimental seja da ordem de $\approx 10^{-2}$, uma vez que a massa do bipolaron é muito maior que a dos elétrons.

Aprimoramos diversos resultados obtidos no conteúdo original [BdPdSP15], começando por: 
Proposição 68 (Bru-Pedra-Pasquale, [BdPdSP15]). Para cada $k \in \mathbb{T}^{2}$ e $h_{b} \in\left[0, \frac{1}{2}\right]$ que fixamos, vale as seguintes sentenças:

a) $\lambda \neq \mathfrak{b}(k)$ é autovalor de $\Xi(k)$ se, e somente se, existe um vetor não nulo $\varphi \in \mathrm{L}^{2}\left(\mathbb{T}^{2}\right)$ no núcleo do operador

$$
\left(\Xi_{1,1}(k)-\lambda \mathbb{l}\right)-(\mathfrak{b}(k)-\lambda)^{-1} \Xi_{1,2}(k) \Xi_{2,1}(k) \in \mathcal{B}\left(\mathrm{L}^{2}\left(\mathbb{T}^{2}\right)\right)
$$

Neste caso, $\lambda$ tem como autovetor associado

$$
\left(\varphi,-(\mathfrak{b}(k)-\lambda)^{-1} \Xi_{2,1}(k) \varphi\right) \in \mathrm{L}^{2}\left(\mathbb{T}^{2}\right) \oplus \mathbb{C}
$$

b) $\mathfrak{b}(k)$ é autovalor de $\Xi(k)$ se, e somente se, $\hat{v}(k)=0$.

Demonstração. Seja $\lambda$ um autovalor de $\Xi(k)$ diferente de $\mathfrak{b}(k)$ e $(\varphi, z)$ um autovetor correspondente. Primeiramente, veja que a equação matricial

$$
\left(\begin{array}{cc}
\Xi_{1,1}(k) & \Xi_{1,2}(k) \\
\Xi_{2,1}(k) & \Xi_{2,2}(k)
\end{array}\right)\left(\begin{array}{l}
\varphi \\
z
\end{array}\right)=\lambda\left(\begin{array}{l}
\varphi \\
z
\end{array}\right)
$$

desponta no sistema linear

$$
\left\{\begin{array}{l}
\left(\Xi_{1,1}(k)-\lambda \mathbb{1}\right) \varphi+\Xi_{1,2}(k) z=0 \\
\Xi_{2,1}(k) \varphi+(\mathfrak{b}(k)-\lambda) z=0
\end{array}\right.
$$

Oras, pela segunda equação

$$
z=-(\mathfrak{b}(k)-\lambda)^{-1} \Xi_{2,1}(k) \varphi
$$

que substituindo na primeira origina

$$
\left(\Xi_{1,1}(k)-\lambda \mathbb{1}\right) \varphi-(\mathfrak{b}(k)-\lambda)^{-1} \Xi_{1,2}(k) \Xi_{2,1}(k) \varphi=0
$$

Note ainda que $\varphi$ não pode ser nulo, do contrário $z$, sendo sua imagem por um certo operador, também se anularia. A recíproca é evidente pois a sucessão lógica acima é uma via de mão dupla. Na sequência,

$$
\hat{v}(k)=0 \Longrightarrow \Xi_{1,2}(k)=0, \Xi_{2,1}(k)=0 \Longrightarrow \Xi(k)(0,1)=\mathfrak{b}(k)(0,1)
$$

Reciprocamente, se $\hat{v}(k)$ é não nulo e $\mathfrak{b}(k)$ persiste como um autovalor de $\Xi(k)$, é porque existe $(f, z) \in$ $\mathrm{L}^{2}\left(\mathbb{T}^{2}\right) \times \mathbb{C}$ não nulo de forma que

$$
\left\{\begin{array}{l}
\left(\Xi_{1,1}(k)-\mathfrak{b}(k) \mathbb{1}\right) f+\Xi_{1,2}(k) z=0 \\
\Xi_{2,1}(k) f=0
\end{array}\right.
$$

A segunda equação indica $f \perp \mathfrak{d}(k)$, e por isso que se aplicamos o funcional $f^{*}$ na primeira, obtém-se

$$
\left\langle f,\left(\Xi_{1,1}(k)-\mathfrak{b}(k) \mathbb{1}\right) f\right\rangle_{2}=0 .
$$

Se $k \neq 0, \mathfrak{b}(k)$ se encontra (estritamente) abaixo do espectro de $\Xi_{1,1}(k), \log \Xi_{1,1}(k)-\mathfrak{b}(k) \mathbb{1}>0$, donde $f=0$. No outro caso, tem-se 


$$
\left\langle f, \mathrm{M}_{\mathfrak{f}(0)} f\right\rangle_{2}+\sum_{\mathbb{Z}^{2}} \mathrm{U}(z)\left\langle f, \mathrm{P}_{z} f\right\rangle_{2}=\left\langle f, \Xi_{1,1}(0) f\right\rangle_{2}=0
$$

mas que por conta da positividade de U e os demais operadores envolvidos, só ocorre quando cada uma das parcelas se anula individualmente. Em particular

$$
0=\left\langle f, \mathrm{M}_{\mathfrak{f}(0)} f\right\rangle_{2}=\int_{\mathbb{T}^{2}}|f|^{2} \mathfrak{f}(0) \mathrm{d} \varrho
$$

e como $\mathfrak{f}(0)$ só se anula na origem do plano euclidiano, de novo $f=0$. De qualquer forma

$$
\Xi_{1,2}(k) z=0
$$

e portanto $z=0$, contradizendo as hipóteses estabelecidas conjuntamente sobre $f$ e $z$.

Para cada $k \in \mathbb{T}^{2}$, ponhamos $\mathfrak{D}_{k} \doteq \mathcal{R}\left(\Xi_{1,1}(k)\right)$. Usando o princípio de Birman-Schwinger, podemos converter o problema de autovalor acima na procura de raízes para uma determinada equação numérica não linear.

Teorema 69 (Bru-Pedra-Pasquale, [BdPdSP15]). Fixe $k \in \mathbb{T}^{2}$ e $h_{b} \in\left[0, \frac{1}{2}\right]$. Então, $\lambda$ é um autovalor de $\Xi(k)$ em $\mathfrak{D}_{k}$ se, e somente se, for solução da equação

$$
\hat{v}(k)^{2} \mathfrak{T}(k, x)+x-\mathfrak{b}(k)=0, \quad x \in \mathcal{R}\left(\Xi_{1,1}(k)\right)
$$

em que

$$
\mathfrak{T}(k, x) \doteq\left\langle\mathfrak{d}(k), \mathcal{R}\left(x ; \Xi_{1,1}(k)\right) \mathfrak{d}(k)\right\rangle_{2}
$$

Demonstração. Trataremos o caso $\hat{v}(k)=0$ em separado. Com efeito, se $\lambda \in \mathfrak{D}_{k}$ é um autovalor de $\Xi(k)$, existe $(f, z) \in \mathrm{L}^{2}\left(\mathbb{T}^{2}\right) \times \mathbb{C}$ não nulo e obedecendo o sistema linear

$$
\left\{\begin{array}{l}
\left(\Xi_{1,1}(k)-\lambda \mathbb{1}\right) f=0 \\
(\mathfrak{b}(k)-\lambda) z=0
\end{array}\right.
$$

Ora, se $\Xi_{1,1}(k)-\lambda \mathbb{1}$ é inversível, $f=0, \log 0 z \neq 0$, e portanto $\lambda-\mathfrak{b}(k)=0$. Aqui o argumento se ramifica em mais dois casos: Se $k \neq 0$,

$$
\hat{v}(k)^{2} \mathfrak{T}(k, \lambda)+\lambda-\mathfrak{b}(k)=0
$$

e a equação está satisfeita por $\lambda$. Reciprocamente, nas condições em que estamos submetidos $\mathfrak{b}(k)$ é a única solução da equação proposta no enunciado, tal como um autovalor de $\Xi(k)$, vide (P.68).

Agora se $k=0$, teríamos $\lambda=\mathfrak{b}(0)=0 \in[0,8 \epsilon] \subseteq \mathbb{R} \backslash \mathfrak{D}_{0}$, e portanto um absurdo causado pela ausência de autovalores de $\Xi(0)$ em $\mathfrak{D}_{0}$. De toda forma,

$$
\hat{v}(0)^{2} \mathfrak{T}(0, x)+x-\mathfrak{b}(0)=0
$$

não possui solução, e o enunciado do teorema também se cumpre neste caso. Por fim, suponhamos $\hat{v}(k) \neq 0$, e para um dado $\lambda \in \mathfrak{D}_{k}$ diferente de $\mathfrak{b}(k)$, defina

$$
V \doteq(\mathfrak{b}(k)-\lambda)^{-1} \Xi_{1,2}(k) \Xi_{2,1}(k) \in \mathcal{B}\left(\mathrm{L}^{2}\left(\mathbb{T}^{2}\right)\right)
$$

Temos

$$
\Xi_{1,2}(k) \Xi_{2,1}(k) \varphi=\hat{v}(k)^{2}\langle\mathfrak{d}(k), \varphi\rangle_{2} \mathfrak{d}(k)
$$


e se $P_{\mathfrak{d}(k)}$ denota o projetor ortogonal de $\mathrm{L}^{2}\left(\mathbb{T}^{2}\right)$ em $\mathbb{C} \mathfrak{d}(k)$, ficamos com

$$
V \varphi=\left(\hat{v}(k)(\mathfrak{b}(k)-\lambda)^{-1 / 2}\|\mathfrak{d}(k)\|_{2} P_{\mathfrak{d}(k)}\right)^{2} \varphi, \quad \varphi \in \mathrm{L}^{2}\left(\mathbb{T}^{2}\right)
$$

Aplicando a (P.68) e (T.25) com

$$
\mathrm{B}(\lambda) \doteq \hat{v}(k)^{2}(\mathfrak{b}(k)-\lambda)^{-1}\|\mathfrak{d}(k)\|_{2}^{2} P_{\mathfrak{d}(k)} \mathcal{R}\left(\lambda ; \Xi_{1,1}(k)\right) P_{\mathfrak{d}(k)}
$$

encontramos

$$
\begin{aligned}
\lambda \text { é autovalor de } \Xi(k) & \Longleftrightarrow \lambda \text { é autovalor de } \Xi_{1,1}(k)-V \\
& \Longleftrightarrow 1 \text { é autovalor de } \mathrm{B}(\lambda) \\
& \Longleftrightarrow \mathrm{B}(\lambda) \mathfrak{d}(k)=\mathfrak{d}(k)
\end{aligned}
$$

esclarecido que todo autovetor $\varphi$ de $\mathrm{B}(\lambda)$ associado ao autovalor 1 é colinear à $\mathfrak{d}(k)$ por efeito da relação

$$
\varphi=\mathrm{B}(\lambda) \varphi \in \mathbb{C} \mathfrak{d}(k)
$$

Advertindo o leitor de que o produto interno de $\mathrm{B}(\lambda) \mathfrak{d}(k)-\mathfrak{d}(k) \operatorname{com} \mathfrak{d}(k)$ só é nulo quando um deles o for (afinal eles são colineares), efetuamos

$$
\begin{aligned}
\mathrm{B}(\lambda) \mathfrak{d}(k)=\mathfrak{d}(k) & \Longleftrightarrow\langle\mathfrak{d}(k), \mathrm{B}(\lambda) \mathfrak{d}(k)-\mathfrak{d}(k)\rangle_{2}=0 \\
& \Longleftrightarrow\langle\mathfrak{d}(k), \mathrm{B}(\lambda) \mathfrak{d}(k)\rangle_{2}=\|\mathfrak{d}(k)\|_{2}^{2} \\
& \Longleftrightarrow \hat{v}(k)^{2} \mathfrak{T}(k, \lambda)=\mathfrak{b}(k)-\lambda
\end{aligned}
$$

completando assim a demonstração.

Corolário 70 (Bru-Pedra-Pasquale, [BdPdSP15]). Seja $k \in \mathbb{T}^{2}$ de modo que $\hat{v}(k) \neq 0$ e fixe $h_{b} \in\left[0, \frac{1}{2}\right]$. Se $\lambda$ é um autovalor de $\Xi(k)$ em $\mathfrak{D}_{k}$, então

$$
\left(\mathrm{L}^{2}\left(\mathbb{T}^{2}\right) \oplus \mathbb{C}\right)_{\lambda}(\Xi(k))=\mathbb{C} \Psi(k)
$$

em que o vetor diretor $\Psi(k)$ é definido por

$$
\Psi(k) \doteq\left(\begin{array}{c}
\hat{v}(k) \mathcal{R}\left(\lambda ; \Xi_{1,1}(k)\right) \mathfrak{d}(k) \\
-1
\end{array}\right) \in \mathrm{L}^{2}\left(\mathbb{T}^{2}\right) \oplus \mathbb{C}
$$

Em particular, todo autovalor de $\Xi(k)$ em $\mathfrak{D}_{k}$ é não degenerado.

Demonstração. Acabamos descobrindo na demonstração do teorema anterior que $\mathfrak{d}(k)$ é base do autoespaço de $\mathrm{B}(\lambda)$ associado ao valor 1. Uma rápida conferida na prova do (T.25) é suficiente para se convencer de que

$$
\varphi_{0} \doteq \hat{v}(k)(\mathfrak{b}(k)-\lambda)^{-\frac{1}{2}}\|\mathfrak{d}(k)\|_{2} \mathcal{R}\left(\lambda ; \Xi_{1,1}(k)\right) \mathfrak{d}(k)
$$

é base do autoespaço de $\Xi_{1,1}(k)-V$ associado ao valor $\lambda$, e pelo (P.68) 


$$
\left(\begin{array}{c}
\varphi_{0} \\
-(\mathfrak{b}(k)-\lambda)^{-1} \Xi_{2,1}(k) \varphi_{0}
\end{array}\right)=\frac{\|\mathfrak{d}(k)\|_{2}}{\sqrt{\mathfrak{b}(k)-\lambda}}\left(\begin{array}{c}
\hat{v}(k) \mathcal{R}\left(\lambda ; \Xi_{1,1}(k)\right) \mathfrak{d}(k) \\
-1
\end{array}\right)
$$

tem que ser base do autoespaço de $\Xi(k)$ associado à $\lambda$. Nas manipulações acima, observe que

$$
\begin{aligned}
-(\mathfrak{b}(k)-\lambda)^{-1} \Xi_{2,1}(k) \varphi_{0} & =-(\mathfrak{b}(k)-\lambda)^{-\frac{3}{2}} \hat{v}(k)^{2}\|\mathfrak{d}(k)\|_{2} \mathfrak{T}(k, \lambda) \\
& =-(\mathfrak{b}(k)-\lambda)^{-\frac{1}{2}}\|\mathfrak{d}(k)\|_{2}
\end{aligned}
$$

afinal de contas $\mathfrak{T}(k, \lambda)=\hat{v}(k)^{-2}(\mathfrak{b}(k)-\lambda)$.

Corolário 71 (Bru-Pedra-Pasquale, [BdPdSP15]). Fixe $h_{b} \in\left[0, \frac{1}{2}\right]$. Para todo $k \in \mathbb{T}^{2}$, há no máximo um autovalor de $\Xi(k)$ em cada componente conexa de $\mathfrak{D}_{k}$.

Demonstração. Dado $x_{0}$ no aberto $\mathfrak{D}_{k}$ e $h \neq 0$ de modo $x_{0}+h \in \mathfrak{D}_{k}$, efetua-se

$$
\begin{aligned}
& \left\|\frac{\mathcal{R}\left(x_{0}+h ; \Xi_{1,1}(k)\right)-\mathcal{R}\left(x_{0} ; \Xi_{1,1}(k)\right)}{h}-\mathcal{R}\left(x_{0} ; \Xi_{1,1}(k)\right)^{2}\right\|_{\mathrm{op}}= \\
& =\left\|\frac{\left\{\left(x_{0}+h\right)-x_{0}\right\} \mathcal{R}\left(x_{0}+h ; \Xi_{1,1}(k)\right) \mathcal{R}\left(x_{0} ; \Xi_{1,1}(k)\right)}{h}-\mathcal{R}\left(x_{0} ; \Xi_{1,1}(k)\right)^{2}\right\|_{\mathrm{op}} \leq \\
& \leq\left\|\mathcal{R}\left(x_{0}+h ; \Xi_{1,1}(k)\right)-\mathcal{R}\left(x_{0} ; \Xi_{1,1}(k)\right)\right\|_{\mathrm{op}}\left\|\mathcal{R}\left(x_{0} ; \Xi_{1,1}(k)\right)\right\|_{\mathrm{op}}
\end{aligned}
$$

por conta da primeira identidade da resolvente. Mas graças a continuidade da aplicação

$$
\mathfrak{D}_{k} \ni x \longmapsto \mathcal{R}\left(x ; \Xi_{1,1}(k)\right) \in \mathcal{B}\left(\mathrm{L}^{2}\left(\mathbb{T}^{2}\right)\right)
$$

fazer $h \rightarrow 0$ ocasiona

$$
\frac{\partial \mathfrak{T}}{\partial x}\left(k, x_{0}\right)=\left\langle\mathfrak{d}(k), \mathcal{R}\left(x_{0} ; \Xi_{1,1}(k)\right)^{2} \mathfrak{d}(k)\right\rangle_{2}=\left\|\mathcal{R}\left(x_{0} ; \Xi_{1,1}(k)\right) \mathfrak{d}(k)\right\|_{2}^{2}>0
$$

afinal $\mathfrak{d}(k)$ é não nulo e $\mathcal{R}\left(x_{0} ; \Xi_{1,1}(k)\right)$ inversível. Por consequência,

$$
\left.\frac{\partial}{\partial x}\left\{\hat{v}(k)^{2} \mathfrak{T}(k, x)+x-\mathfrak{b}(k)\right\}\right|_{x=x_{0}}=\hat{v}(k)^{2}\left\|\mathcal{R}\left(x_{0} ; \Xi_{1,1}(k)\right) \mathfrak{d}(k)\right\|_{2}^{2}+1>0
$$

ou seja,

$$
\mathfrak{D}_{k} \ni x \longmapsto \hat{v}(k)^{2} \mathfrak{T}(k, x)+x-\mathfrak{b}(k) \in \mathbb{R}
$$

é estritamente crescente em cada componente conexa de $\mathfrak{D}_{k}$ (pois recorde que o Teorema do Valor Medio só funciona em intervalos), podendo cruzar a origem no máximo uma vez em cada uma delas.

Seja dito que para todo momento não nulo $k$, é possível identificar a existência genuína de um autovalor para a fibra $\Xi(k)$ ao menos na componente conexa inferior de $\mathfrak{D}_{k}$. Este é o conteúdo que emana do seguinte corolário: 
Corolário 72 (Bru-Pedra-Pasquale, [BdPdSP15]). Seja $k \in \mathbb{T}_{0}^{2}$ e $h_{b} \in\left[0, \frac{1}{2}\right]$. Existe um, e apenas um autovalor $\mathrm{E}(k)$ de $\Xi(k)$ estritamente menor que $4 \epsilon-2 \epsilon \cos (k / 2)$. Neste caso,

$$
\begin{cases}\mathrm{E}(k)<\mathfrak{b}(k) & , \hat{v}(k) \neq 0 \\ \mathrm{E}(k)=\mathfrak{b}(k) & , \hat{v}(k)=0\end{cases}
$$

Demonstração. De acordo com o corolário acima, a semi reta $(-\infty, 4 \epsilon-2 \epsilon \cos (k / 2))$ deve abrigar no máximo um autovalor de $\Xi(k)$. Quando $\hat{v}(k)=0$, já sabemos que este indivíduo é $\mathfrak{b}(k)$. Caso contrário, note que

$$
x<4 \epsilon-2 \epsilon \cos (k / 2)=\min \sigma\left(\Xi_{1,1}(k)\right) \Longrightarrow \mathcal{R}\left(x ; \Xi_{1,1}(k)\right)>0 \Longrightarrow \mathfrak{T}(k, x)>0
$$

e por consequência

$$
\hat{v}(k)^{2} \mathfrak{T}(k, x)+x-\mathfrak{b}(k)>0, \quad x \in[\mathfrak{b}(k), 4 \epsilon-2 \epsilon \cos (k / 2))
$$

Isso nos certifica de que $\Xi(k)$ não possui autovalor no intervalo acima, e agora resta inspecionar $(-\infty, \mathfrak{b}(k))$. Com efeito,

$$
\begin{aligned}
|\mathfrak{T}(k, x)| \leq\|\mathfrak{d}(k)\|_{2}^{2}\left\|\mathcal{R}\left(x ; \Xi_{1,1}(k)\right)\right\|_{\text {op }}=\|\mathfrak{d}(k)\|_{2}^{2} \mathrm{~d}\left(x, \sigma\left(\Xi_{1,1}(k)\right)\right)^{-1}= \\
\quad=\|\mathfrak{d}(k)\|_{2}^{2}|x-(4 \epsilon-2 \epsilon \cos (k / 2))|^{-1} \leq\|\mathfrak{d}(k)\|_{2}^{2}|x-\mathfrak{b}(k)|^{-1}
\end{aligned}
$$

sempre que $x<\mathfrak{b}(k)$, e por isso

$$
\lim _{x \rightarrow-\infty} \mathfrak{T}(k, x)=0
$$

ou seja, $\mathfrak{T}(k, x)$ fica arbitrariamente pequeno à medida que $x$ decresce, e com isso a equação original adquire um comportamento assintótico delineado pela reta $x-\mathfrak{b}(k)$, intitulada como sua assíntota oblíqua. Em suma,

$$
\lim _{x \rightarrow-\infty}\left\{\hat{v}(k)^{2} \mathfrak{T}(k, x)+x-\mathfrak{b}(k)\right\}=-\infty
$$

Por outro lado, resulta da continuidade de $\mathfrak{T}(k, \cdot): \mathfrak{D}_{k} \longrightarrow \mathbb{R}$ no ponto $\mathfrak{b}(k)$ que

$$
\lim _{x \rightarrow \mathfrak{b}(k)}\left\{\hat{v}(k)^{2} \mathfrak{T}(k, x)+x-\mathfrak{b}(k)\right\}=\hat{v}(k)^{2} \mathfrak{T}(k, \mathfrak{b}(k))>0
$$

e assim encontramos $r>0$ de forma que

$$
\hat{v}(k)^{2} \mathfrak{T}(k, x)+x-\mathfrak{b}(k)>0 \quad \forall x \in(\mathfrak{b}(k)-r, \mathfrak{b}(k)+r), x \neq \mathfrak{b}(k)
$$

Pelo teorema do valor intermediário, a equação em pauta cruza o eixo das abscissas em algum valor $\mathrm{E}(k) \in(-\infty, \mathfrak{b}(k))$. 


\subsection{Relação de Dispersão de Pares Ligado com Baixa Energia}

"O investimento em conhecimento sempre paga os melhores juros."

Benjamin Franklin ${ }^{14}$

Definição 73 (Relação de Dispersão). Uma função $\omega: \mathbb{T}_{0}^{2} \longrightarrow \mathbb{R}$ diz-se uma relação de dispersão quando cumpre as seguintes condições:

a) Para todo momento $k \in \mathbb{T}^{2}, \omega(k)$ é autovalor de $\Xi(k)$.

b) $\omega$ é pelo menos de classe $\mathscr{C}^{2}$ no interior de $\mathbb{T}_{0}^{2}$

Em vista disso, o corolário anterior sugere que busquemos por condições que assegure um grau de regularidade satisfatório para E. Ora, o grau de regularidade de

$$
\Phi(k, x) \doteq \hat{v}(k)^{2} \mathfrak{T}(k, x)+x-\mathfrak{b}(k)
$$

definida no aberto ${ }^{15}$

$$
\Omega \doteq\left\{(k, x): k \in \operatorname{int}\left(\mathbb{T}_{0}^{2}\right), x \in \mathfrak{D}_{k}\right\}
$$

de $\mathbb{R}^{3}$ depende única e exclusivamente de $\hat{v}$ porque todas as demais aplicações envolvidas em sua definição são reais analíticas. Como consequência:

Teorema 74. Seja $h_{b} \in\left[0, \frac{1}{2}\right]$. A aplicação E tem mesmo grau de regularidade que $\hat{v}$ no interior de $\mathbb{T}_{0}^{2}$.

Demonstração. Devemos mostrar que E é ao menos contínua pois $\hat{v}$ sempre é. Com efeito, a continuidade de $\Xi$ resulta na continuidade de

$$
\mathbb{T}^{2} \ni k \stackrel{\theta}{\longrightarrow} \min \sigma(\Xi(k)) \in \mathbb{R}
$$

e portanto de $\mathrm{E}=\theta \uparrow \mathbb{T}_{0}^{2}$ em razão da (P.3). Agora, suponha que $\hat{v}$ seja de classe $\mathscr{C}^{d}(\operatorname{com} d \neq 1)$ no interior de $\mathbb{T}_{0}^{2}$ e fixe $\kappa \in \operatorname{int}\left(\mathbb{T}_{0}^{2}\right)$. Tendo em vista que

- $\Phi \in \mathrm{C}^{d}(\Omega)$

- $\Phi(\kappa, \mathrm{E}(\kappa))=0$

- $\frac{\partial \Phi}{\partial x}(\kappa, \mathrm{E}(\kappa))=\hat{v}(\kappa)^{2}\left\|\mathcal{R}\left(\mathrm{E}(\kappa) ; \Xi_{1,1}(\kappa)\right) \mathfrak{d}(\kappa)\right\|_{2}^{2}+1>0$

o teorema da função implícita prevê a existência de dois abertos $U \subseteq \mathbb{R}^{2}$ e $J \subseteq \mathbb{R}$ de forma que

$$
(\kappa, \mathrm{E}(\kappa)) \in U \times J \subseteq \Omega
$$

\footnotetext{
${ }^{14}$ Benjamin Franklin, $\odot(17 / 01 / 1706)-\dagger(17 / 04 / 1790)$

${ }^{15}$ Confira o apêndice A.
} 
e para todo momento $k \in U$, existe um único número real $\xi(k) \in J$ satisfazendo implicitamente a equação

$$
\Phi(k, \xi(k))=0
$$

O teorema também nos diz que $\xi: U \longrightarrow \mathbb{R}$, assim definida, possui mesmo grau de regularidade que $\Phi$, sendo portanto de classe $\mathscr{C}^{d}$. Agora, como

$$
\xi(\kappa)=\mathrm{E}(\kappa)<4 \epsilon-2 \epsilon \cos (\kappa / 2)
$$

por continuidade deve existir uma vizinhança aberta $\mathcal{A} \subseteq \operatorname{int}\left(\mathbb{T}_{0}^{2}\right)$ de $\kappa$ onde

$$
\xi(k)<4 \epsilon-2 \epsilon \cos (k / 2) \quad \forall k \in \mathcal{A}
$$

Mas sendo $\mathrm{E}(k)$ o único autovalor de $\Xi(k)$ estritamente menor que $4 \epsilon-2 \epsilon \cos (k / 2)$, segue

$$
\mathrm{E} \uparrow \mathcal{A}=\xi \uparrow \mathcal{A} \in \mathrm{C}^{d}(\mathcal{A})
$$

Pela arbitrariedade na escolha de $\kappa$, mostramos que E é localmente $\mathscr{C}^{d}$ em cada ponto interior de seu domínio, e portanto globalmente $\mathscr{C}^{d}$ em int $\left(\mathbb{T}_{0}^{2}\right)$.

Quando $\hat{v}$ for pelo menos de classe $\mathscr{C}^{2}$, a conclusão é de que E define uma relação de dispersão que denominamos relação de dispersão de pares ligado com baixa energia (típicamente $d$-wave ${ }^{16}$ ). A partir daí, outras grandezas físicas podem ser consideradas, como por exemplo:

Definição 75 (Velocidade de Grupo e Tensor de Massa). Seja $h_{b} \in\left[0, \frac{1}{2}\right]$. Se vé pelo menos de classe $\mathscr{C}^{2}$ no interior de $\mathbb{T}_{0}^{2}$, então dizemos que

$$
\nu^{*}(k) \doteq \vec{\nabla} \mathrm{E}(k)
$$

é a velocidade de grupo e

$$
m^{*}(k) \doteq \operatorname{Hess}[\mathrm{E}](k)^{-1}
$$

o tensor de massa (de pares ligado com baixa energia) associados ao momento $k \in \mathbb{T}_{0}^{2}$.

Vale lembrar que a regularidade de $\hat{v}$ pode ser obtida atribuindo-se condições de somabilidade na própria função de acoplamento. Com efeito, a teoria geral das transformadas de Fourier estipula que se $x^{\alpha} v(x)$ for $\mathbb{Z}^{2}$-somável para todo multi-índice $\alpha$ de ordem 2 e comprimento $k \in \mathbb{N}_{0}$, então $\hat{v} \in \mathrm{C}^{k}\left(\mathbb{R}^{2}\right)$, valendo

$$
\partial^{\alpha} \hat{v}=(-\imath)^{|\alpha|} \widehat{x^{\alpha} v(x)}
$$

Na demonstração do teorema anterior, como E e $\xi$ coincidem em toda uma vizinhança aberta do ponto $\kappa$, também vale

$$
\frac{\partial \mathrm{E}}{\partial k_{i}}(\kappa)=\frac{\partial \xi}{\partial k_{i}}(\kappa)=-\frac{\frac{\partial \Phi}{\partial k_{i}}(\kappa, \xi(\kappa))}{\frac{\partial \Phi}{\partial x}(\kappa, \xi(\kappa))}=-\left(\frac{\partial \Phi}{\partial x}(\kappa, \mathrm{E}(\kappa))\right)^{-1} \frac{\partial \Phi}{\partial k_{i}}(\kappa, \mathrm{E}(\kappa))
$$

e por consequência

\footnotetext{
${ }^{16}$ Como mostra o artigo [BdSPDdP19], tal relação de dispersão dá origem a pares ligado do tipo $d$-wave quando se ajusta o modelo com os parâmetros prototípicos do cuprato supercondutor LaSr 214 e leva-se em conta repulsões de alcançe zero com alta magnitude. [Veja a (D.78) à frente.]
} 


$$
\nu^{*}(k)=-\left(\frac{\partial \Phi}{\partial x}(k, \mathrm{E}(k))\right)^{-1} \vec{\nabla}_{k} \Phi(k, \mathrm{E}(k)), \quad k \in \mathbb{T}_{0}^{2}
$$

Uma fórmula explícita para o tensor de massa pode ser obtida derivando-se a expressão acima. Com base nisso, o artigo [BdSPDdP19] soluciona o paradoxo da massa mostrando que o tensor de massa de pares com baixa energia $m^{*}$ corresponde de fato a uma massa pequena, quando calculado com parâmetros de cuprato. O que acontece é que o composto com dispersão E formado por bipolaron e elétrons tem massa efetiva pequena, apesar da grande massa "livre" do bipolaron que entra nesse composto; contabilizada na teoria quando assumimos $h_{b}$ pequeno.

Desconsiderando o acoplamento bipolaron-elétron como no modelo de Hubbard $(v \equiv 0)$, note que $\mathrm{E}=\mathfrak{b} \uparrow \mathbb{T}_{0}^{2}$, ou seja, o estado ligado presente no baixo do espectro é precisamente a energia cinética do bipolaron, de modo que a partícula efetiva com baixa energia é o próprio bipolaron livre. Como já comentamos, este caso onde o bipolaron não interage com ninguém e não forma nenhum estado composto não possui interesse físico devido a sua grande massa.

Definição 76 (Energia Fundamental). $E_{0} \doteq \min \sigma(\hat{H})$ é dita ser a energia fundamental do sistema.

Fisicamente, os valores espectrais de $\hat{H}$ representam os níveis energéticos que se encontram à disposição do sistema em questão, e daí a terminologia. No teorema subsequente, fornecemos uma demonstração alternativa aquela apresentada nos artigos base que não remeta à teoria geral de Kato-Birman.

Teorema 77 (Bru-Pedra-Pasquale, [BdPdSP15]). $E_{0}=\min _{k \in \mathbb{T}^{2}} \min \sigma(\Xi(k))$

Demonstração. Antes de tudo, vamos mostrar que a reunião dos espectros das fibras $\Xi(k)$ é fechado em $\mathbb{R}$. Com efeito, se

$$
\lambda \in \overline{\bigcup_{k \in \mathbb{T}^{2}} \sigma(\Xi(k))}
$$

existe uma sequência $\left(\lambda_{n}\right)_{n \in \mathbb{N}}$ convergindo à $\lambda$ em que cada

$$
\lambda_{n} \in \sigma\left(\Xi\left(k_{n}\right)\right), \quad k_{n} \in \mathbb{T}^{2}
$$

Em virtude da compacidade de $\mathbb{T}^{2}$, podemos assumir (por passagem a uma subsequência) que os $k_{n}$ converge para um determinado $k_{0} \in \mathbb{T}^{2}$. Aplicando a continuidade de $\Xi$, obtemos

$$
\|\cdot\|_{\text {op }}-\lim _{n \rightarrow \infty} \Xi\left(k_{n}\right)-\lambda_{n} \mathbb{1}=\Xi\left(k_{0}\right)-\lambda \mathbb{1}
$$

donde $\lambda \in \sigma\left(\Xi\left(k_{0}\right)\right)$, caso contrário $\Xi\left(k_{n}\right)-\lambda_{n} \mathbb{1}$ seria inversível para $j$ suficientemente grande ${ }^{17}$. Pelo (T.40), segue

$$
\mu \notin \bigcup_{k \in \mathbb{T}^{2}} \sigma(\Xi(k)) \Longrightarrow \exists r>0: \mathbf{B}_{r}(\mu) \cap \sigma(\Xi(k))=\varnothing \quad \forall k \in \mathbb{T}^{2} \Longrightarrow \mu \notin \sigma(\hat{H})
$$

e por contra positiva

\footnotetext{
${ }^{17}$ Os operadores limitados e inversíveis atuando em um espaço de Banach $\mathcal{X}$ formam um subconjunto aberto de $\mathcal{B}(X)$ (relativamente a norma de operador).
} 


$$
\sigma(\hat{H}) \subseteq \bigcup_{k \in \mathbb{T}^{2}} \sigma(\Xi(k)) \Longrightarrow \inf _{k \in \mathbb{T}^{2}} \min \sigma(\Xi(k)) \leq E_{0}
$$

Pela continuidade de $\theta$ (definida no teorema anterior) e compacidade de $\mathbb{T}^{2}$, note que

$$
\theta(\kappa)=\inf _{k \in \mathbb{T}^{2}} \min \sigma(\Xi(k))
$$

para algum $\kappa \in \mathbb{T}^{2}$, de forma que para mostrarmos a desigualdade inversa, basta provar que $\theta(\kappa) \in \sigma(\hat{H})$. Com efeito, para todo $\varepsilon>0$ a continuidade de $\theta$ no ponto $\kappa$ nos fornece $\delta>0$ para o qual

$$
k \in \mathbb{T}^{2}:|k-\kappa|<\delta \Longrightarrow \theta(k) \in \sigma(\Xi(k)) \cap(\theta(\kappa)-\varepsilon, \theta(\kappa)+\varepsilon)
$$

implicando

$$
\varrho\left(\left\{k \in \mathbb{T}^{2}: \sigma(\Xi(k)) \cap(\theta(\kappa)-\varepsilon, \theta(\kappa)+\varepsilon) \neq \varnothing\right\}\right) \geq \varrho\left(\mathbb{T}^{2} \cap \mathbf{B}_{\delta}(\kappa)\right)>0
$$

donde a tese, vide (T.40). 


\section{Capítulo 5}

\section{Comportamento Efetivo no Limite de Hard-Core da Repulsão Eletrônica}

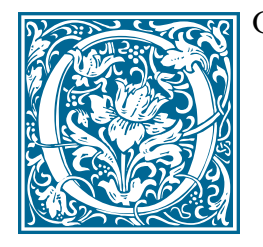

CASO de maior notoriedade e amplamente analisado nos artigos base ocorre no regime em que um elétron sofre a repulsão provocada pela sua contra-parte apenas quando estes se encontram na mesma célula elementar da rede cristalina. Entitulada como repulsão eletrônica de alcance zero por razões óbvias, neste capítulo analisamos o que acontece quando sua magnitude tende ao infinito. Fisicamente, este limite impede que os elétrons ocupem o mesmo ponto da rede de forma simultânea. Com base em tudo isso, ao final do capítulo formalizamos um modelo efetivo de baixas energias que não dependa mais, de detalhes, da interação repulsiva entre os elétrons com o fim de possibilitar uma abordagem mais simples ao efeito coletivo de supercondução.

\subsection{Representando $\mathfrak{T}$ em Termos de Integrais Explícitas}

"No meio das dificuldades encontra-se a oportunidade."

Albert Einstein

Definição 78 (Repulsão Eletrônica de Alcance Zero). Dizemos que a repulsão eletrônica U é de alcance zero quando assume a forma

$$
\mathrm{U}(z)=\delta_{0, z} U_{0}
$$

em que a constante $U_{0} \geq 0$ chama-se magnitude de $\mathrm{U}$.

Nesta situação,

$$
\Xi_{1,1}\left(U_{0}, k\right)=\mathrm{M}_{\mathfrak{f}(k)}+U_{0} \mathrm{P}_{0}
$$

em que $\mathrm{P}_{0}$ designa o projetor ortogonal de $\mathrm{L}^{2}\left(\mathbb{T}^{2}\right)$ na direção de $\mathfrak{s} \equiv 1$. Fixado $k \in \mathbb{T}^{2}$ e $\lambda$ fora da imagem de $\mathfrak{f}(k)$, considere as seguintes quantidades, facilmente exploradas com cálculo numérico:

$$
\mathfrak{R}_{\mathfrak{s}, \mathfrak{s}}^{(0)} \doteq \frac{1}{(2 \pi)^{2}} \int_{-\pi}^{\pi} \int_{-\pi}^{\pi} \frac{1}{\epsilon\{4-\cos (h-k)-\cos (h)\}-\lambda} \mathrm{d} h_{1} \mathrm{~d} h_{2}
$$




$$
\begin{aligned}
& \mathfrak{R}_{\mathfrak{s}, \mathfrak{d}}^{(0)} \doteq \frac{2}{(2 \pi)^{2}} \int_{-\pi}^{\pi} \int_{-\pi}^{\pi} \frac{\cos (h-k)+\cos (2 h-k)+1}{\epsilon\{4-\cos (h-k)-\cos (h)\}-\lambda} \mathrm{d} h_{1} \mathrm{~d} h_{2} \\
& \mathfrak{R}_{\mathfrak{d}, \mathfrak{d}}^{(0)} \doteq \frac{4}{(2 \pi)^{2}} \int_{-\pi}^{\pi} \int_{-\pi}^{\pi} \frac{|\cos (h-k)+\cos (2 h-k)+1|^{2}}{\epsilon\{4-\cos (h-k)-\cos (h)\}-\lambda} \mathrm{d} h_{1} \mathrm{~d} h_{2}
\end{aligned}
$$

Para alcançar nossos objetivos, é preciso estender mais alguns resultados teóricos obtidos nos artigos base, a começar pelo:

Teorema 79 (Bru-Pedra-Pasquale, [BdPdSP15]). Seja $k \in \mathbb{T}^{2}, U_{0} \geq 0$ e $\lambda<4 \epsilon-2 \epsilon \cos (k / 2)$. Então,

$$
\mathfrak{T}\left(U_{0}, k, \lambda\right)=\frac{\mathfrak{R}_{\mathfrak{d}, \mathfrak{d}}^{(0)}}{1+U_{0} \mathfrak{R}_{\mathfrak{s}, \mathfrak{s}}^{(0)}}+U_{0} \frac{\mathfrak{R}_{\mathfrak{d}, \mathfrak{d}}^{(0)} \mathfrak{R}_{\mathfrak{s}, \mathfrak{s}}^{(0)}-\left(\mathfrak{R}_{\mathfrak{s}, \mathfrak{d}}^{(0)}\right)^{2}}{1+U_{0} \mathfrak{R}_{\mathfrak{s}, \mathfrak{s}}^{(0)}}
$$

onde

$$
\frac{\mathfrak{R}_{\mathfrak{d}, \mathfrak{d}}^{(0)}}{1+U_{0} \mathfrak{R}_{\mathfrak{s}, \mathfrak{s}}^{(0)}}>0 \quad, \quad \frac{\mathfrak{R}_{\mathfrak{d}, \mathfrak{d}}^{(0)} \mathfrak{R}_{\mathfrak{s}, \mathfrak{s}}^{(0)}-\left(\mathfrak{R}_{\mathfrak{s}, \mathfrak{d}}^{(0)}\right)^{2}}{1+U_{0} \mathfrak{R}_{\mathfrak{s}, \mathfrak{s}}^{(0)}}>0
$$

Demonstração. De início, defina as seguintes quantidades auxiliares

$$
\mathfrak{R}_{\mathfrak{s}, \mathfrak{s}} \doteq\left\langle\mathfrak{s}, \mathcal{R}\left(\lambda ; \Xi_{1,1}\left(U_{0}, k\right)\right) \mathfrak{s}\right\rangle_{2} \quad, \quad \mathfrak{R}_{\mathfrak{d}, \mathfrak{d}} \doteq \mathfrak{T}\left(U_{0}, k, \lambda\right) \quad, \quad \mathfrak{R}_{\mathfrak{s}, \mathfrak{d}} \doteq\left\langle\mathfrak{s}, \mathcal{R}\left(\lambda ; \Xi_{1,1}\left(U_{0}, k\right)\right) \mathfrak{d}(k)\right\rangle_{2}
$$

Pela segunda identidade da resolvente, temos

$$
\begin{array}{r}
\mathcal{R}\left(\lambda ; \Xi_{1,1}\left(U_{0}, k\right)\right)-\mathcal{R}\left(\lambda ; \mathrm{M}_{\mathfrak{f}(k)}\right)=\mathcal{R}\left(\lambda ; \Xi_{1,1}\left(U_{0}, k\right)\right)\left(\mathrm{M}_{\mathfrak{f}(k)}-\Xi_{1,1}\left(U_{0}, k\right)\right) \mathcal{R}\left(\lambda ; \mathrm{M}_{\mathfrak{f}(k)}\right)= \\
=-U_{0} \mathcal{R}\left(\lambda ; \Xi_{1,1}\left(U_{0}, k\right)\right) \mathrm{P}_{0} \mathcal{R}\left(\lambda ; \mathrm{M}_{\mathfrak{f}(k)}\right)
\end{array}
$$

Avaliando a expressão acima em $\mathfrak{s}$, e aplicando o funcional $\mathfrak{s}^{*}$ no resultado, obtemos

$$
\mathfrak{R}_{\mathfrak{s}, \mathfrak{s}}=\mathfrak{R}_{\mathfrak{s}, \mathfrak{s}}^{(0)}-U_{0}\left\langle\mathfrak{s}, \mathcal{R}\left(\lambda ; \Xi_{1,1}(k)\right) \mathrm{P}_{0} \mathcal{R}\left(\lambda ; \mathrm{M}_{\mathfrak{f}(k)}\right) \mathfrak{s}\right\rangle_{2}
$$

Com relação ao produto interno remanescente, efetua-se

$$
\begin{aligned}
\left\langle\mathcal{R}\left(\lambda ; \Xi_{1,1}(k)\right) \mathfrak{s}, \mathrm{P}_{0} \mathcal{R}\left(\lambda ; \mathrm{M}_{\mathfrak{f}(k)}\right) \mathfrak{s}\right\rangle_{2}=\sum_{z \in \mathbb{Z}^{2}}\left\langle\mathcal{R}\left(\lambda ; \Xi_{1,1}(k)\right) \mathfrak{s}, E_{z}\right\rangle_{2}\left\langle E_{z}, \mathrm{P}_{0} \mathcal{R}\left(\lambda ; \mathrm{M}_{\mathfrak{f}(k)}\right) \mathfrak{s}\right\rangle_{2}= \\
=\left\langle\mathcal{R}\left(\lambda ; \Xi_{1,1}(k)\right) \mathfrak{s}, \mathfrak{s}\right\rangle_{2}\left\langle\mathfrak{s}, \mathrm{P}_{0} \mathcal{R}\left(\lambda ; \mathrm{M}_{\mathfrak{f}(k)}\right) \mathfrak{s}\right\rangle_{2}=\mathfrak{R}_{\mathfrak{s}, \mathfrak{s}} \mathfrak{R}_{\mathfrak{s}, \mathfrak{s}}^{(0)}
\end{aligned}
$$

Repetindo este mecanismo mais algumas vezes, chegamos as equações

$$
\begin{aligned}
& \mathfrak{R}_{\mathfrak{s , \mathfrak { s }}}=\mathfrak{R}_{\mathfrak{s}, \mathfrak{s}}^{(0)}-U_{0} \mathfrak{R}_{\mathfrak{s}, \mathfrak{s}} \mathfrak{R}_{\mathfrak{s}, \mathfrak{s}}^{(0)} \\
& \mathfrak{R}_{\mathfrak{d}, \mathfrak{d}}=\mathfrak{R}_{\mathfrak{d}, \mathfrak{d}}^{(0)}-U_{0} \mathfrak{R}_{\mathfrak{s}, \mathfrak{d}} \mathfrak{R}_{\mathfrak{s}, \mathfrak{d}}^{(0)} \\
& \mathfrak{R}_{\mathfrak{s}, \mathfrak{d}}=\mathfrak{R}_{\mathfrak{s}, \mathfrak{d}}^{(0)}-U_{0} \mathfrak{R}_{\mathfrak{s}, \mathfrak{d}} \mathfrak{R}_{\mathfrak{s}, \mathfrak{s}}^{(0)}
\end{aligned}
$$




$$
\mathfrak{R}_{\mathfrak{s}, \mathfrak{d}}=\mathfrak{R}_{\mathfrak{s}, \mathfrak{d}}^{(0)}-U_{0} \mathfrak{R}_{\mathfrak{s}, \mathfrak{s}} \mathfrak{R}_{\mathfrak{s}, \mathfrak{d}}^{(0)}
$$

que reescritas em termos matriciais, nos confere

$$
\left(\begin{array}{cc}
U_{0} \mathfrak{R}_{\mathfrak{s}, \mathfrak{s}}^{(0)}+1 & 0 \\
U_{0} \mathfrak{R}_{\mathfrak{s}, \mathfrak{d}}^{(0)} & 1
\end{array}\right)\left(\begin{array}{cc}
\mathfrak{R}_{\mathfrak{s}, \mathfrak{s}} & \mathfrak{R}_{\mathfrak{s}, \mathfrak{d}} \\
\mathfrak{R}_{\mathfrak{s}, \mathfrak{d}} & \mathfrak{R}_{\mathfrak{d}, \mathfrak{d}}
\end{array}\right)=\left(\begin{array}{cc}
\mathfrak{R}_{\mathfrak{s}, \mathfrak{s}}^{(0)} & \mathfrak{R}_{\mathfrak{s}, \mathfrak{d}}^{(0)} \\
\mathfrak{R}_{\mathfrak{s}, \mathfrak{d}}^{(0)} & \mathfrak{R}_{\mathfrak{d}, \mathfrak{d}}^{(0)}
\end{array}\right)
$$

Pelas hipóteses atribuídas a $\lambda, \mathcal{R}\left(\lambda ; \mathrm{M}_{\mathfrak{f}(k)}\right)>0$, logo $\mathfrak{R}_{\mathfrak{s}, \mathfrak{s}}^{(0)}>0$, portanto

$$
\operatorname{det}\left(\begin{array}{cc}
U_{0} \mathfrak{R}_{\mathfrak{s}, \mathfrak{s}}^{(0)}+1 & 0 \\
U_{0} \mathfrak{R}_{\mathfrak{s}, \mathfrak{d}}^{(0)} & 1
\end{array}\right)=U_{0} \mathfrak{R}_{\mathfrak{s}, \mathfrak{s}}^{(0)}+1>0
$$

o que a torna inversível. Assim,

$$
\left(\begin{array}{ll}
\mathfrak{R}_{\mathfrak{s}, \mathfrak{s}} & \mathfrak{R}_{\mathfrak{s}, \mathfrak{d}} \\
\mathfrak{R}_{\mathfrak{s}, \mathfrak{d}} & \mathfrak{R}_{\mathfrak{d}, \mathfrak{d}}
\end{array}\right)=\frac{1}{U_{0} \mathfrak{R}_{\mathfrak{s}, \mathfrak{s}}^{(0)}+1}\left(\begin{array}{cc}
1 & 0 \\
-U_{0} \mathfrak{R}_{\mathfrak{s}, \mathfrak{d}}^{(0)} & U_{0} \mathfrak{R}_{\mathfrak{s}, \mathfrak{s}}^{(0)}+1
\end{array}\right)\left(\begin{array}{cc}
\mathfrak{R}_{\mathfrak{s}, \mathfrak{s}}^{(0)} & \mathfrak{R}_{\mathfrak{s}, \mathfrak{d}}^{(0)} \\
\mathfrak{R}_{\mathfrak{s}, \mathfrak{d}}^{(0)} & \mathfrak{R}_{\mathfrak{d}, \mathfrak{d}}^{(0)}
\end{array}\right)
$$

e em particular

$$
\mathfrak{T}\left(U_{0}, k, \lambda\right)=\mathfrak{R}_{\mathfrak{d}, \mathfrak{d}}=\frac{\mathfrak{R}_{\mathfrak{d}, \mathfrak{d}}^{(0)}\left(U_{0} \mathfrak{R}_{\mathfrak{s}, \mathfrak{s}}^{(0)}+1\right)-U_{0}\left(\mathfrak{R}_{\mathfrak{s}, \mathfrak{d}}^{(0)}\right)^{2}}{U_{0} \mathfrak{R}_{\mathfrak{s}, \mathfrak{s}}^{(0)}+1}
$$

Por fim, se $P$ denota o projetor ortogonal no subespaço gerado por $\mathfrak{s}$ e $\mathfrak{d}(k)$, defina

$$
Q \doteq\left(P \mathcal{R}\left(\lambda ; \mathrm{M}_{\mathfrak{f}(k)}\right) P\right) \uparrow\langle\mathfrak{d}(k), \mathfrak{s}\rangle
$$

Note que $\mathfrak{g} \doteq \mathfrak{d}(k)-2 \mathfrak{s} \perp \mathfrak{s}$, e por isso

$$
Q \mathfrak{s}=\frac{1}{\|\mathfrak{g}\|_{2}^{2}}\left\langle\mathfrak{g}, \mathcal{R}\left(\lambda ; \mathrm{M}_{\mathfrak{f}(k)}\right) \mathfrak{s}\right\rangle_{2} \mathfrak{g}+\left\langle\mathfrak{s}, \mathcal{R}\left(\lambda ; \mathrm{M}_{\mathfrak{f}(k)}\right) \mathfrak{s}\right\rangle_{2} \mathfrak{s}=\frac{1}{\|\mathfrak{g}\|_{2}^{2}}\left(\mathfrak{R}_{\mathfrak{s}, \mathfrak{d}}^{(0)}-2 \mathfrak{R}_{\mathfrak{s}, \mathfrak{s}}^{(0)}\right) \mathfrak{g}+\mathfrak{R}_{\mathfrak{s}, \mathfrak{s}}^{(0)} \mathfrak{s}
$$

Do mesmo modo,

$$
Q \mathfrak{g}=\frac{1}{\|\mathfrak{g}\|_{2}^{2}}\left\langle\mathfrak{g}, \mathcal{R}\left(\lambda ; \mathrm{M}_{\mathfrak{f}(k)}\right) \mathfrak{g}\right\rangle_{2} \mathfrak{g}+\left\langle\mathfrak{s}, \mathcal{R}\left(\lambda ; \mathrm{M}_{\mathfrak{f}(k)}\right) \mathfrak{g}\right\rangle_{2} \mathfrak{s}=\frac{1}{\|\mathfrak{g}\|_{2}^{2}}\left(\mathfrak{R}_{\mathfrak{d}, \mathfrak{d}}^{(0)}+4 \mathfrak{R}_{\mathfrak{s , \mathfrak { s }}}^{(0)}-4 \mathfrak{R}_{\mathfrak{s}, \mathfrak{d}}^{(0)}\right) \mathfrak{g}+\left(\mathfrak{R}_{\mathfrak{s}, \mathfrak{d}}^{(0)}-2 \mathfrak{R}_{\mathfrak{s}, \mathfrak{s}}^{(0)}\right) \mathfrak{s}
$$

e finalmente

$$
[Q]_{\mathcal{B}}=\left(\begin{array}{cc}
\frac{1}{\|\mathfrak{g}\|_{2}^{2}}\left(\mathfrak{R}_{\mathfrak{d}, \mathfrak{d}}^{(0)}+4 \mathfrak{R}_{\mathfrak{s}, \mathfrak{s}}^{(0)}-4 \mathfrak{R}_{\mathfrak{s}, \mathfrak{d}}^{(0)}\right) & \mathfrak{R}_{\mathfrak{s}, \mathfrak{d}}^{(0)}-2 \mathfrak{R}_{\mathfrak{s}, \mathfrak{s}}^{(0)} \\
\frac{1}{\|\mathfrak{g}\|_{2}^{2}}\left(\mathfrak{R}_{\mathfrak{s}, \mathfrak{d}}^{(0)}-2 \mathfrak{R}_{\mathfrak{s}, \mathfrak{s}}^{(0)}\right) & \mathfrak{R}_{\mathfrak{s , \mathfrak { s }}}^{(0)}
\end{array}\right)
$$

onde $\mathcal{B}=\{\mathfrak{d}(k)-2 \mathfrak{s}, \mathfrak{s}\}$. Oras, $Q$ é restrição de uma composição de operadores positivo, e logo positivo. Contudo, se existisse $\varphi$ em seu domínio para o qual $\langle\varphi, Q \varphi\rangle_{2}$ fosse nulo, teríamos

$$
\left\langle\varphi, \mathcal{R}\left(\lambda ; \mathrm{M}_{\mathfrak{f}(k)}\right) \varphi\right\rangle_{2}=\left\langle P \varphi, \mathcal{R}\left(\lambda ; \mathrm{M}_{\mathfrak{f}(k)}\right) P \varphi\right\rangle_{2}=\left\langle\varphi, P \mathcal{R}\left(\lambda ; \mathrm{M}_{\mathfrak{f}(k)}\right) P \varphi\right\rangle_{2}=0
$$

o que contraria a positividade estrita de $\mathcal{R}\left(\lambda ; \mathrm{M}_{\mathfrak{f}(k)}\right)$. Logo $Q>0$, e por consequência 


$$
0<\operatorname{det}(Q)=\frac{1}{\|\mathfrak{g}\|_{2}^{2}}\left\{\mathfrak{R}_{\mathfrak{s}, \mathfrak{s}}^{(0)}\left(\mathfrak{R}_{\mathfrak{d}, \mathfrak{d}}^{(0)}+4 \mathfrak{R}_{\mathfrak{s , \mathfrak { s }}}^{(0)}-4 \mathfrak{R}_{\mathfrak{s}, \mathfrak{d}}^{(0)}\right)-\left(\mathfrak{R}_{\mathfrak{s}, \mathfrak{d}}^{(0)}-2 \mathfrak{R}_{\mathfrak{s}, \mathfrak{s}}^{(0)}\right)^{2}\right\}=\frac{1}{\|\mathfrak{g}\|_{2}^{2}}\left\{\mathfrak{R}_{\mathfrak{s}, \mathfrak{s}}^{(0)} \mathfrak{R}_{\mathfrak{d}, \mathfrak{d}}^{(0)}-\left(\mathfrak{R}_{\mathfrak{s}, \mathfrak{d}}^{(0)}\right)^{2}\right\}
$$

Isso conclui a demonstração.

Corolário 80 (Bru-Pedra-Pasquale, [BdPdSP15]). Para todo $k \in \mathbb{T}^{2}, U_{0} \geq 0$ e $\lambda<4 \epsilon-$ $2 \epsilon \cos (k / 2)$, tem-se

$$
\left|\mathfrak{T}\left(U_{0}, k, \lambda\right)-\frac{\mathfrak{R}_{\mathfrak{d}, \mathfrak{d}}^{(0)} \mathfrak{R}_{\mathfrak{s}, \mathfrak{s}}^{(0)}-\left(\mathfrak{R}_{\mathfrak{s}, \mathfrak{d}}^{(0)}\right)^{2}}{\mathfrak{R}_{\mathfrak{s}, \mathfrak{s}}^{(0)}}\right|<10^{2} U_{0}^{-1}
$$

Demonstração. De fato, pois como $\left|\mathfrak{R}_{\mathfrak{s}, \mathfrak{d}}^{(0)}\right| \leq 10 \mathfrak{R}_{\mathfrak{s}, \mathfrak{s}}^{(0)}$, então

$$
\mathfrak{T}\left(U_{0}, k, \lambda\right)-\frac{\mathfrak{R}_{\mathfrak{d}, \mathfrak{d}}^{(0)} \mathfrak{R}_{\mathfrak{s , ~}, \mathfrak{s}}^{(0)}-\left(\mathfrak{R}_{\mathfrak{s}, \mathfrak{d}}^{(0)}\right)^{2}}{\mathfrak{R}_{\mathfrak{s}, \mathfrak{s}}^{(0)}}=\frac{\left(\mathfrak{R}_{\mathfrak{s}, \mathfrak{d}}^{(0)}\right)^{2}}{\left(U_{0} \mathfrak{R}_{\mathfrak{s}, \mathfrak{s}}^{(0)}+1\right) \mathfrak{R}_{\mathfrak{s}, \mathfrak{s}}^{(0)}} \leq \frac{10^{2} \mathfrak{R}_{\mathfrak{s}, \mathfrak{s}}^{(0)}}{U_{0} \mathfrak{R}_{\mathfrak{s}, \mathfrak{s}}^{(0)}+1}<10^{2} U_{0}^{-1}
$$

e com isso a tese.

A independência da estimativa acima com relação a escolha de $k$ e $\lambda$ será crucial no que se segue.

\subsection{Modelo Efetivo de Baixa Energia para o Limite de Hard- Core}

"Daria tudo que sei pela metade do que ignoro."

René Descartes ${ }^{1}$

Definição 81 (Relação de Dispersão Efetiva). Seja $\omega:[0,+\infty) \times \mathbb{T}_{0}^{2} \longrightarrow \mathbb{R}$ uma relação de dispersão dependente da força de repulsão $U_{0}$. Dizemos que $\omega^{(\infty)}: \mathbb{T}_{0}^{2} \longrightarrow \mathbb{R}$ é a relação de dispersão efetiva associado a $\omega$ no limite de hard-core quando:

a) Para todo $k \in \mathbb{T}_{0}^{2}, \omega^{(\infty)}(k)=\lim _{U_{0} \rightarrow \infty} \omega\left(U_{0}, k\right)$.

b) $\omega^{(\infty)}$ é pelo menos de classe $\mathscr{C}^{2}$ no interior de $\mathbb{T}_{0}^{2}$.

Dado um significado preciso a quantidade que estamos procurando, antecipamos que

$$
\mathrm{E}^{(\infty)}(k) \doteq \sup _{U_{0} \geq 0} \mathrm{E}\left(U_{0}, k\right), \quad k \in \mathbb{T}_{0}^{2}
$$

apenas por economia de notação.

\footnotetext{
${ }^{1}$ René Descartes, $\odot(31 / 03 / 1596)-\dagger(11 / 02 / 1650)$
} 
Definição 82 (Energia Fundamental Efetiva). A energia fundamental efetiva do sistema é definida por

$$
E_{0}^{(\infty)} \doteq \lim _{U_{0} \rightarrow \infty} E_{0}\left(U_{0}\right)
$$

Perceba que o item c) à frente evidencia a estabilidade do vetor diretor que define o autoespaço da fibra $\Xi\left(U_{0}, k\right)$ associado ao valor $\mathrm{E}\left(U_{0}, k\right)$ com relação ao limite hard-core.

Teorema 83. Seja $h_{b} \in\left[0, \frac{1}{2}\right]$. São verdadeiras as seguintes afirmações:

a) Para todo $k \in \mathbb{T}_{0}^{2}$, tem-se

$$
\lim _{U_{0} \rightarrow \infty} \mathrm{E}\left(U_{0}, k\right)=\sup _{U_{0} \geq 0} \mathrm{E}\left(U_{0}, k\right) \leq \mathfrak{b}(k)
$$

b) O sistema possui uma energia fundamental efetiva, valendo

$$
E_{0}^{(\infty)}=\sup _{U_{0} \geq 0} E_{0}\left(U_{0}\right) \leq 0
$$

c) Para todo $k \in \mathbb{T}_{0}^{2}$, existe o seguinte limite:

$$
\text { S- } \lim _{U_{0} \rightarrow \infty} \mathcal{R}\left(\mathrm{E}\left(U_{0}, k\right) ; \Xi_{1,1}\left(U_{0}, k\right)\right)
$$

Demonstração. Para todo $0 \leq U_{0} \leq V_{0}$ e $k \in \mathbb{T}^{2}$, tem-se

$$
\Xi_{1,1}\left(V_{0}, k\right)-\Xi_{1,1}\left(U_{0}, k\right)=\left(V_{0}-U_{0}\right) \mathrm{P}_{0} \geq 0
$$

$\operatorname{logo} \Xi\left(U_{0}, k\right) \leq \Xi\left(V_{0}, k\right)$, e portanto

$$
\theta\left(U_{0}, k\right) \leq \theta\left(V_{0}, k\right) \leq \mathfrak{b}(k)
$$

vide (C.4). Por um lado, isso mostra que cada $\mathrm{E}(\cdot, k)$ é monótona crescente, limitada supreriormente, e daí segue a). Por outro, que se minimizamos a desigualdade acima em $\mathbb{T}^{2}$, conseguimos

$$
E_{0}\left(U_{0}\right) \leq E_{0}\left(V_{0}\right) \leq 0
$$

por onde segue b). Na sequência, computa-se

$$
\begin{aligned}
& \left\|\mathcal{R}\left(\mathrm{E}^{(\infty)}(k) ; \Xi_{1,1}\left(U_{0}, k\right)\right)-\mathcal{R}\left(\mathrm{E}\left(U_{0}, k\right) ; \Xi_{1,1}\left(U_{0}, k\right)\right)\right\|_{\text {op }} \leq \\
& \leq\left|\mathrm{E}^{(\infty)}(k)-\mathrm{E}\left(U_{0}, k\right)\right| \cdot\left\|\mathcal{R}\left(\mathrm{E}^{(\infty)}(k) ; \Xi_{1,1}\left(U_{0}, k\right)\right)\right\|\left\|_{\text {op }}\right\| \mathcal{R}\left(\mathrm{E}\left(U_{0}, k\right) ; \Xi_{1,1}\left(U_{0}, k\right)\right) \|_{\text {op }}= \\
& =\left|\mathrm{E}^{(\infty)}(k)-\mathrm{E}\left(U_{0}, k\right)\right| \cdot\left\|\mathcal{R}\left(\mathrm{E}^{(\infty)}(k) ; \Xi_{1,1}\left(U_{0}, k\right)\right)\right\|_{\text {op }}^{2}= \\
& \quad=\frac{\left|\mathrm{E}^{(\infty)}(k)-\mathrm{E}\left(U_{0}, k\right)\right|}{\left|\mathrm{E}^{(\infty)}(k)-(4 \epsilon-2 \epsilon \cos (k / 2))\right|^{2}} \stackrel{U_{0} \rightarrow \infty}{\longrightarrow} 0
\end{aligned}
$$

por efeito da primeira identidade da resolvente e maior proximidade de $\mathrm{E}^{(\infty)}(k)$ com relação ao espectro de $\Xi_{1,1}\left(U_{0}, k\right)$ do que $\mathrm{E}\left(U_{0}, k\right)$. Em palavras, provamos que um dos limites abaixo existe se, e somente se, o outro existe, sendo eles iguais, em caso afirmativo: 


$$
\|\cdot\|_{\text {op }}{ }^{-} \lim _{U_{0} \rightarrow \infty} \mathcal{R}\left(\mathrm{E}\left(U_{0}, k\right) ; \Xi_{1,1}\left(U_{0}, k\right)\right)=\|\cdot\|_{\text {op }}{ }^{-} \lim _{U_{0} \rightarrow \infty} \mathcal{R}\left(\mathrm{E}^{(\infty)}(k) ; \Xi_{1,1}\left(U_{0}, k\right)\right)
$$

Oras, como $\left\{\Xi_{1,1}\left(U_{0}, k\right)\right\}_{U_{0} \geq 0}$ é uma família monótona crescente de operadores estritamente positivos, então

$$
\left\{\mathcal{R}\left(\mathrm{E}^{(\infty)}(k) ; \Xi_{1,1}\left(U_{0}, k\right)\right): U_{0} \geq 0\right\}
$$

é uma família monótona decrescente de operadores estritamente positivos, e daí segue c).

Teorema 84. Seja $h_{b} \in\left[0, \frac{1}{2}\right]$. São verdadeiras as seguintes asserções:

a) Para todo $k \in \mathbb{T}_{0}^{2}$,

$$
\mathrm{E}^{(\infty)}(k)+\hat{v}(k)^{2} \frac{\mathfrak{R}_{\mathfrak{d}, \mathfrak{d}}^{(0)} \mathfrak{R}_{\mathfrak{s}, \mathfrak{s}}^{(0)}-\left(\mathfrak{R}_{\mathfrak{s}, \mathfrak{d}}^{(0)}\right)^{2}}{\mathfrak{R}_{\mathfrak{s}, \mathfrak{s}}^{(0)}}=\mathfrak{b}(k)
$$

Bem entendido, as quantidades $\mathfrak{R}^{(0)}$. agora são dadas relativamente $\grave{a} \lambda=\mathrm{E}^{(\infty)}(k)$.

b) Igualmente a função E, verifica-se

$$
\begin{cases}\mathrm{E}^{(\infty)}(k)<\mathfrak{b}(k) & , \hat{v}(k) \neq 0 \\ \mathrm{E}^{(\infty)}(k)=\mathfrak{b}(k) & , \hat{v}(k)=0\end{cases}
$$

c) Se $\hat{v}$ é de classe $\mathscr{C}^{d}($ com $d \neq 1)$ no interior de $\mathbb{T}_{0}^{2}, \mathrm{E}^{(\infty)}$ também será.

Demonstração. Não há o que provar se $\hat{v}(k)=0$, e do contrário aplicamos o (C.80) para obter

$$
\left|\frac{\mathfrak{b}(k)-\mathrm{E}\left(U_{0}, k\right)}{\hat{v}(k)^{2}}-\frac{\mathfrak{R}_{\mathfrak{d}, \mathfrak{d}}^{(0)} \mathfrak{R}_{\mathfrak{s , s}}^{(0)}-\left(\mathfrak{R}_{\mathfrak{s}, \mathfrak{d}}^{(0)}\right)^{2}}{\mathfrak{R}_{\mathfrak{s}, \mathfrak{s}}^{(0)}}\right|=\left|\mathfrak{T}\left(U_{0}, k, \mathrm{E}\left(U_{0}, k\right)\right)-\frac{\mathfrak{R}_{\mathfrak{d}, \mathfrak{d}}^{(0)} \mathfrak{R}_{\mathfrak{s , \mathfrak { s }}}^{(0)}-\left(\mathfrak{R}_{\mathfrak{s}, \mathfrak{d}}^{(0)}\right)^{2}}{\mathfrak{R}_{\mathfrak{s}, \mathfrak{s}}^{(0)}}\right|<10^{2} U_{0}^{-1}
$$

lembrando que as quantidades $\mathfrak{R}^{(0)}$. estão dadas em função de $\lambda=\mathrm{E}\left(U_{0}, k\right)$. Equivalentemente, tem-se

$$
\left|\mathfrak{b}(k)-\mathrm{E}\left(U_{0}, k\right)-\hat{v}(k)^{2} \frac{\mathfrak{R}_{\mathfrak{d}, \mathfrak{d}}^{(0)} \mathfrak{R}_{\mathfrak{s}, \mathfrak{s}}^{(0)}-\left(\mathfrak{R}_{\mathfrak{s}, \mathfrak{d}}^{(0)}\right)^{2}}{\mathfrak{R}_{\mathfrak{s}, \mathfrak{s}}^{(0)}}\right|<10^{2} U_{0}^{-1} \hat{v}(k)^{2}
$$

para todo $U_{0} \geq 0$, e assim

$$
\lim _{U_{0} \rightarrow \infty}\left\{\mathrm{E}\left(U_{0}, k\right)+\hat{v}(k)^{2} \frac{\mathfrak{R}_{\mathfrak{d}, \mathfrak{d}}^{(0)} \mathfrak{R}_{\mathfrak{s}, \mathfrak{s}}^{(0)}-\left(\mathfrak{R}_{\mathfrak{s}, \mathfrak{d}}^{(0)}\right)^{2}}{\mathfrak{R}_{\mathfrak{s}, \mathfrak{s}}^{(0)}}\right\}=\mathfrak{b}(k)
$$

Pela continuidade da resolvente de $\mathrm{M}_{\mathfrak{f}(k)}$, a expressão acima também tem como limite o lado esquerdo da relação que se deseja mostrar, e daí segue a). Para o item b), basta observar que

$$
\mathfrak{R}_{\mathfrak{d}, \mathfrak{d}}^{(0)} \mathfrak{R}_{\mathfrak{s}, \mathfrak{s}}^{(0)}-\left(\mathfrak{R}_{\mathfrak{s}, \mathfrak{d}}^{(0)}\right)^{2}>0
$$

conforme foi mostrado no (T.79). Enfim, provemos c). Com efeito,

$$
F(k, x) \doteq x+\hat{v}(k)^{2} \frac{\mathfrak{R}_{\mathfrak{d}, \mathfrak{d}}^{(0)} \mathfrak{R}_{\mathfrak{s}, \mathfrak{s}}^{(0)}-\left(\mathfrak{R}_{\mathfrak{s}, \mathfrak{d}}^{(0)}\right)^{2}}{\mathfrak{R}_{\mathfrak{s}, \mathfrak{s}}^{(0)}}-\mathfrak{b}(k)
$$


definida no aberto

$$
\mathcal{A} \doteq\left\{(k, x): k \in \operatorname{int}\left(\mathbb{T}_{0}^{2}\right), x \in \mathcal{R}\left(\mathrm{M}_{\mathfrak{f}(k)}\right)\right\}
$$

tem mesmo grau de regularidade que $\hat{v}$ por conta da real analiticidade das demais aplicações que encontramse envolvidas em sua definição, sendo assim

$$
F \in \mathrm{C}^{d}(\mathcal{A})
$$

Para todo $(k, x) \in \mathcal{A}$, temos

$$
\left\langle\mathfrak{s}, \mathcal{R}\left(x ; \mathrm{M}_{\mathfrak{f}(k)}\right)^{2} \mathfrak{d}(k)\right\rangle_{2}=\left\langle\mathcal{R}\left(x ; \mathrm{M}_{\mathfrak{f}(k)}\right) \mathfrak{s}, \mathcal{R}\left(x ; \mathrm{M}_{\mathfrak{f}(k)}\right) \mathfrak{d}(k)\right\rangle_{2} \leq\left\|\mathcal{R}\left(x ; \mathrm{M}_{\mathfrak{f}(k)}\right) \mathfrak{s}\right\|_{2}\left\|\mathcal{R}\left(x ; \mathrm{M}_{\mathfrak{f}(k)}\right) \mathfrak{d}(k)\right\|_{2}
$$

$\log 0$

$$
\begin{aligned}
& \left(\mathfrak{R}_{\mathfrak{s}, \mathfrak{s}}^{(0)}\right)^{2} \frac{\partial}{\partial x}\left(\frac{\mathfrak{R}_{\mathfrak{d}, \mathfrak{d}}^{(0)} \mathfrak{R}_{\mathfrak{s}, \mathfrak{s}}^{(0)}-\left(\mathfrak{R}_{\mathfrak{s}, \mathfrak{d}}^{(0)}\right)^{2}}{\mathfrak{R}_{\mathfrak{s}, \mathfrak{s}}^{(0)}}\right)(k, x)=\left\{\partial_{x}\left(\mathfrak{R}_{\mathfrak{d}, \mathfrak{d}}^{(0)}\right) \mathfrak{R}_{\mathfrak{s}, \mathfrak{s}}^{(0)}+\mathfrak{R}_{\mathfrak{d}, \mathfrak{d}}^{(0)} \partial_{x}\left(\mathfrak{R}_{\mathfrak{s , ~}, \mathfrak{s}}^{(0)}\right)-2 \mathfrak{R}_{\mathfrak{s}, \mathfrak{d}}^{(0)} \partial_{x}\left(\mathfrak{R}_{\mathfrak{s}, \mathfrak{d}}^{(0)}\right)\right\} \mathfrak{R}_{\mathfrak{s}, \mathfrak{s}}^{(0)}+ \\
& -\partial_{x}\left(\mathfrak{R}_{\mathfrak{s}, \mathfrak{s}}^{(0)}\right)\left\{\mathfrak{R}_{\mathfrak{d}, \mathfrak{d}}^{(0)} \mathfrak{R}_{\mathfrak{s , ~}, \mathfrak{s}}^{(0)}-\left(\mathfrak{R}_{\mathfrak{s}, \mathfrak{d}}^{(0)}\right)^{2}\right\}=\left(\mathfrak{R}_{\mathfrak{s , \mathfrak { s }}}^{(0)}\right)^{2} \partial_{x}\left(\mathfrak{R}_{\mathfrak{d}, \mathfrak{d}}^{(0)}\right)-2 \mathfrak{R}_{\mathfrak{s}, \mathfrak{d}}^{(0)} \mathfrak{R}_{\mathfrak{s , \mathfrak { s }}}^{(0)} \partial_{x}\left(\mathfrak{R}_{\mathfrak{s}, \mathfrak{d}}^{(0)}\right)+\left(\mathfrak{R}_{\mathfrak{s}, \mathfrak{d}}^{(0)}\right)^{2} \partial_{x}\left(\mathfrak{R}_{\mathfrak{s}, \mathfrak{s}}^{(0)}\right) \geq \\
& \geq\left(\left\|\mathcal{R}\left(x ; \mathrm{M}_{\mathfrak{f}(k)}\right) \mathfrak{d}(k)\right\|_{2} \mathfrak{R}_{\mathfrak{s}, \mathfrak{s}}^{(0)}-\left\|\mathcal{R}\left(x ; \mathrm{M}_{\mathfrak{f}(k)}\right) \mathfrak{s}\right\|_{2} \mathfrak{R}_{\mathfrak{s}, \mathfrak{d}}^{(0)}\right)^{2}
\end{aligned}
$$

portanto

$$
\frac{\partial F}{\partial x}(k, x) \geq 1+\hat{v}(k)^{2}\left(\frac{\left\|\mathcal{R}\left(x ; \mathrm{M}_{\mathfrak{f}(k)}\right) \mathfrak{d}(k)\right\|_{2} \mathfrak{R}_{\mathfrak{s}, \mathfrak{s}}^{(0)}-\left\|\mathcal{R}\left(x ; \mathrm{M}_{\mathfrak{f}(k)}\right) \mathfrak{s}\right\|_{2} \mathfrak{R}_{\mathfrak{s , d}}^{(0)}}{\mathfrak{R}_{\mathfrak{s}, \mathfrak{s}}^{(0)}}\right)^{2}>0
$$

e com isso $F(k, \cdot)$ cruza a origem no máximo uma vez em cada um dos intervalos $(-\infty, 4 \epsilon-2 \epsilon \cos (k / 2))$ e $(4 \epsilon+2 \epsilon \cos (k / 2),+\infty)$. A partir daqui, basta repetir o que foi feito no (T.74).

Quando $\hat{v}$ for pelo menos de classe $\mathscr{C}^{2}$, é natural que denominemos a relação de dispersão efetiva $\mathrm{E}^{(\infty)}$ associada a E com o sufixo de pares ligado com baixa energia, em concordância com o caso ordinário. Observe também o caráter espontâneo de $\mathrm{E}^{(\infty)}$, uma vez que o modelo não exige condições adicionais para que a dispersão E admita uma versão efetiva.

Definição 85 (Velocidade de Grupo e Tensor de Massa Efetivo). Seja $h_{b} \in\left[0, \frac{1}{2}\right]$. Se $\hat{v}$ é pelo menos de classe $\mathscr{C}^{2}$ no interior de $\mathbb{T}_{0}^{2}$, então dizemos que

$$
\nu_{\infty}^{*}(k) \doteq \vec{\nabla} \mathrm{E}^{(\infty)}(k)
$$

é a velocidade de grupo efetiva e

$$
m_{\infty}^{*}(k) \doteq \operatorname{Hess}\left[\mathrm{E}^{(\infty)}\right](k)^{-1}
$$

o tensor de massa efetivo (de pares ligado com baixa energia) associado ao momento $k \in \mathbb{T}_{0}^{2}$.

Reforçamos que a presença de um modelo efetivo é uma qualidade do regime de baixa energia: Simulações numéricas divulgadas em [BdSPDdP19] também revelam a existência de um autovalor $\mathrm{F}\left(U_{0}, k\right)$ para a fibra $\Xi\left(U_{0}, k\right)$ acima de seu espectro essencial, quando a magnitude $U_{0}$ é suficientemente grande. Isso dá origem a pares respulsivamente ligados, sendo inclusive reportado em experimentos recentes, vide [WTL $\left.{ }^{+} 06\right]$ e [DD06]. Por essa razão, denomina-se F como relação de dispersão de pares repulsivamente ligado, mas que por efeito de 


$$
\lim _{U_{0} \rightarrow \infty} \mathrm{F}\left(U_{0}, k\right)=+\infty \quad \forall k \in \mathbb{T}^{2}
$$

obtido via cálculo numérico, não conduz a formação de um modelo efetivo (de alta energia no caso). Feitas estas considerações, encerra-se mais um capítulo. 


\section{Capítulo 6}

\section{Canais de Espalhamento}

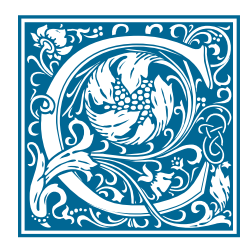

ANAL DE ESPALHAMENTo, ou ainda, canal dispersivo, é o nome que atribuímos ao espalhamento de um sistema referente a uma determinada dinâmica livre, para grandes tempos $(t \rightarrow \pm \infty)$. Neste capítulo, aplica-se a teoria abstrata desenvolvida na seção 3.2 para se estudar dois tipos de canais de espalhamento que aparecem no sistema em questão. O primeiro deles sugere que para grandes tempos, a dinâmica do sistema adquire um comportamento livre de forças repulsivas e interações de acoplamento, causado pelo afastamento progressivo de um elétron com relação ao outro. Em contraste, o segundo estabelece que os eletrons permanecem ligados durante todo instante de tempo, com uma função de onda que descreve a posição relativa dos dois que é constante no tempo. Além disso, o movimento do centro de gravidade dos dois é o de uma partícula livre com dispersão E.

\subsection{Canal de Pares Eletrônicos Não-Ligado}

"Toda grande caminhada começa com um simples passo."

Siddharta Gautama ${ }^{1}$

Para esta seção, considere os seguintes operadores auxiliares:

$$
\begin{array}{cc}
J: \int_{\mathbb{T}^{2}}^{\oplus} \mathrm{L}^{2}\left(\mathbb{T}^{2}\right) \mathrm{d} \varrho \longrightarrow \int_{\mathbb{T}^{2}}^{\oplus} \mathrm{L}^{2}\left(\mathbb{T}^{2}\right) \oplus \mathbb{C} \mathrm{d} \varrho & J^{*}: \int_{\mathbb{T}^{2}}^{\oplus} \mathrm{L}^{2}\left(\mathbb{T}^{2}\right) \oplus \mathbb{C} \mathrm{d} \varrho \longrightarrow \int_{\mathbb{T}^{2}}^{\oplus} \mathrm{L}^{2}\left(\mathbb{T}^{2}\right) \mathrm{d} \varrho \\
\Psi \longmapsto(\Psi, 0) & \Psi=\left(\Psi_{1}, \Psi_{2}\right) \longmapsto \Psi_{1}
\end{array}
$$

Nosso primeiro objetivo é determinar o projetor no espaço absolutamente contínuo $P_{\mathrm{ac}}$ de uma série de operadores, começando por $\Xi_{2,2}(k)$. Com efeito, se $G$ é um boreliano de $\mathbb{R}$ com medida de Lebesgue nula e contendo o ponto $\mathfrak{b}(k)$, então para todo $z \in \mathbb{C}$ diferente de zero, tem-se

$$
\Omega_{z}(G)=\left\langle z, P_{G}\left(\Xi_{2,2}(k)\right) z\right\rangle_{\mathbb{C}}=|z|^{2} \chi_{G}(\mathfrak{b}(k)) \neq 0 \Longrightarrow \mathbb{C}_{\mathrm{ac}}\left(\Xi_{2,2}(k)\right)=\{0\} \Longrightarrow P_{\mathrm{ac}}\left(\Xi_{2,2}(k)\right)=0
$$

Pelo (Ex.42), também já sabemos que $P_{\mathrm{ac}}\left(\mathrm{M}_{\mathfrak{f}(k)}\right)=\mathbb{1}$, e daí

$$
\int_{\mathbb{T}^{2}}^{\oplus} P_{\mathrm{ac}}\left(\mathrm{M}_{\mathfrak{f}(k)} \oplus \Xi_{2,2}(k)\right) \mathrm{d} \varrho=\int_{\mathbb{T}^{2}}^{\oplus} P_{\mathrm{ac}}\left(\mathrm{M}_{\mathfrak{f}(k)}\right) \oplus P_{\mathrm{ac}}\left(\Xi_{2,2}(k)\right) \mathrm{d} \varrho=J J^{*}
$$

\footnotetext{
${ }^{1}$ Siddharta Gautama, $\odot 563$ a.C - † 483 a.C (datas incertas)
} 
graças também a (P.15). Com a notação do $\left(\right.$ Ex.39), enfim vamos mostrar $P_{\mathrm{ac}}\left(\mathcal{L}_{\mathrm{f}}\right)=\mathbb{1}$. Com efeito, se $\Psi$ é um vetor na integral direta de $L^{2}\left(\mathbb{T}^{2}\right)$ e $B$ um boreliano de $\mathbb{R}$ com medida de Lebesgue nula, então

$$
\begin{array}{r}
\Omega_{\Psi, \mathcal{L}_{\mathfrak{f}}}(B)=\left\langle\Psi, \mathrm{P}_{B}\left(\int_{\mathbb{T}^{2}}^{\oplus} \mathrm{M}_{\mathfrak{f}(h)} \mathrm{d} \varrho(h)\right) \Psi\right\rangle_{2, \mathrm{~L}^{2}\left(\mathbb{T}^{2}\right)}=\left\langle\Psi,\left(\int_{\mathbb{T}^{2}}^{\oplus} \mathrm{P}_{B}\left(\mathrm{M}_{\mathfrak{f}(h)}\right) \mathrm{d} \varrho(h)\right) \Psi\right\rangle_{2, \mathrm{~L}^{2}\left(\mathbb{T}^{2}\right)}= \\
=\int_{\mathbb{T}^{2}}\left\langle\Psi(k),\left(\int_{\mathbb{T}^{2}}^{\oplus} \mathrm{P}_{B}\left(\mathrm{M}_{\mathfrak{f}(h)}\right) \mathrm{d} \varrho(h)\right) \Psi(k)\right\rangle_{2} \mathrm{~d} \varrho(k)=\int_{\mathbb{T}^{2}}\left\langle\Psi(k), \mathrm{P}_{B}\left(\mathrm{M}_{\mathfrak{f}(k)}\right) \Psi(k)\right\rangle_{2} \mathrm{~d} \varrho(k)= \\
=\int_{\mathbb{T}^{2}} \Omega_{\Psi(k), \mathrm{M}_{\mathfrak{f}(k)}}(B) \mathrm{d} \varrho(k)=0
\end{array}
$$

dado que pelo (Ex.42), $\Psi(k) \in \mathrm{L}^{2}\left(\mathbb{T}^{2}\right)_{\text {ac }}\left(\mathrm{M}_{\mathfrak{f}(k)}\right)$ para todo $k \in \mathbb{T}^{2}$, inevitavelmente. Sendo $B$ arbitrário, concluímos que $\Psi$ se encontra no espaço absolutamente contínuo de $\mathcal{L}_{\mathfrak{f}}$, exatamente o que queríamos provar.

O próximo resultado diz que para grandes tempos, a dinâmica gerada por $\hat{H}$ torna-se muito similar aquela induzida pelo termo cinético de $H_{f}$. A existência deste canal de espalhamento é esperado, uma vez que as interações presentes no sistema enfraquecem à medida que a distância entre os elétrons cresce.

Teorema 86. Verifica-se a seguinte identidade:

$$
W^{ \pm}\left(\int_{\mathbb{T}^{2}}^{\oplus} \Xi(k) \mathrm{d} \varrho(k), \int_{\mathbb{T}^{2}}^{\oplus} \mathrm{M}_{\mathfrak{f}(k)} \mathrm{d} \varrho(k) ; J\right) J^{*}=\int_{\mathbb{T}^{2}}^{\oplus} \Omega^{ \pm}\left(\Xi(k), \mathrm{M}_{\mathfrak{f}(k)} \oplus \Xi_{2,2}(k)\right) \mathrm{d} \varrho(k)
$$

Demonstração. Com efeito,

$$
\Xi(k)-\mathrm{M}_{\mathrm{f}(k)} \oplus \Xi_{2,2}(k) \in \mathscr{S}_{1}\left(\mathrm{~L}^{2}\left(\mathbb{T}^{2}\right) \oplus \mathbb{C}\right)
$$

porque é compacto, e $\Omega^{ \pm}\left(\Xi(k), \mathrm{M}_{\mathfrak{f}(k)} \oplus \Xi_{2,2}(k)\right)$ existe por conta do (C.59). Aplicando o (T.37), segue

$$
\begin{aligned}
\int_{\mathbb{T}^{2}}^{\oplus} \Omega^{ \pm}\left(\Xi(k), \mathrm{M}_{\mathfrak{f}(k)} \oplus \Xi_{2,2}(k)\right) \mathrm{d} \varrho(k) & =\mathrm{s}-\lim _{t \rightarrow \mp \infty} \int_{\mathbb{T}^{2}}^{\oplus} e^{\imath t \Xi(k)} e^{-\imath t\left(\mathrm{M}_{\mathfrak{f}(k)} \oplus \Xi_{2,2}(k)\right)} P_{\mathrm{ac}}\left(\mathrm{M}_{\mathfrak{f}(k)} \oplus \Xi_{2,2}(k)\right) \mathrm{d} \varrho(k) \\
& =\mathrm{s}-\lim _{t \rightarrow \mp \infty}\left(\int_{\mathbb{T}^{2}}^{\oplus} e^{\imath t \Xi(k)} \mathrm{d} \varrho(k)\right)\left(\int_{\mathbb{T}^{2}}^{\oplus} e^{-\imath t\left(\mathrm{M}_{\mathfrak{f}(k)} \oplus \Xi_{2,2}(k)\right)} \mathrm{d} \varrho(k)\right) J J^{*}
\end{aligned}
$$

que junto de

$$
\left(\int_{\mathbb{T}^{2}}^{\oplus} e^{-\imath t\left(\mathrm{M}_{\mathfrak{f}(k)} \oplus \Xi_{2,2}(k)\right)} \mathrm{d} \varrho(k)\right) J J^{*}=J\left(\int_{\mathbb{T}^{2}}^{\oplus} e^{-\imath t \mathrm{M}_{\mathfrak{f}(k)}} \mathrm{d} \varrho(k)\right) J^{*}
$$


acarreta em

$$
\begin{aligned}
W^{ \pm}\left(\int_{\mathbb{T}^{2}}^{\oplus} \Xi(k) \mathrm{d} \varrho(k), \int_{\mathbb{T}^{2}}^{\oplus} \mathrm{M}_{\mathfrak{f}(k)} \mathrm{d} \varrho(k) ; J\right) J & =\mathrm{s}-\lim _{t \rightarrow \mp \infty} \exp \left(\imath t \int_{\mathbb{T}^{2}}^{\oplus} \Xi(k) \mathrm{d} \varrho(k)\right) J \exp \left(-\imath t \int_{\mathbb{T}^{2}}^{\oplus} \mathrm{M}_{\mathfrak{f}(k)} \mathrm{d} \varrho(k)\right) J^{*} \\
& =\mathrm{s}-\lim _{t \rightarrow \mp \infty}\left(\int_{\mathbb{T}^{2}}^{\oplus} e^{\imath t \Xi(k)} \mathrm{d} \varrho(k)\right) J\left(\int_{\mathbb{T}^{2}}^{\oplus} e^{\left.-\imath t \mathrm{M}_{\mathfrak{f}(k)} \mathrm{d} \varrho(k)\right) J^{*}}\right. \\
& =\int_{\mathbb{T}^{2}}^{\oplus} \Omega^{ \pm}\left(\Xi(k), \mathrm{M}_{\mathfrak{f}(k)} \oplus \Xi_{2,2}(k)\right) \mathrm{d} \varrho(k)
\end{aligned}
$$

e portanto a tese.

\subsection{Canal de Pareamento $d$-Wave}

"Se eu vi mais longe, foi por estar sobre ombros de gigantes."

Isaac Newton

Por questões meramente estéticas, em alguns casos escreveremos $\mathcal{H} \equiv \mathrm{L}^{2}\left(\mathbb{T}^{2}\right) \oplus \mathbb{C}$.

Definição 87 (Operador de Entrelaçamento). Sejam $A$ e $B$ operadores lineares agindo nos $K$-espaços vetoriais $\mathcal{X}$ y, respectivamente. Dizemos que $C \in \mathrm{L}(\mathcal{Y}, \mathcal{X})$ entrelaça $A$ e $B$ se for não nulo, e verifica

$$
A C=C B
$$

É fácil ver que a imagem de $C$ é subespaço $A$-invariante de $X$, pois

$$
\psi \in \operatorname{Im}(C) \Longrightarrow \exists \varphi \in y: \psi=C \varphi \Longrightarrow A \psi=A C \varphi=C B \varphi \in \operatorname{Im}(C)
$$

Observamos outros fatos relevantes acerca desta noção na seguinte proposição:

Proposição 88. Sejam $A$ e $B$ operadores autoadjuntos e limitados agindo nos espaços de Hilbert $\mathfrak{H}$ e $\mathcal{H}$, respectivamente. Se $J \in \mathcal{B}(\mathcal{H}, \mathfrak{H})$ entrelaça $A$ e $B$, são verdadeiras as seguintes afirmações:

a) Para toda $f \in \mathfrak{B}_{\mathrm{c}}(\mathbb{R})$, vale

$$
f(A) J=J f(B)
$$

o que significa que $J$ também entrelaça $f(A)$ e $f(B)$.

b) O par $(A, B)$ admite o operador de onda de Kato associado a identificação J, e neste caso

$$
W^{ \pm}(A, B ; J)=J P_{\mathrm{ac}}(B)
$$

Demonstração. Primeiramente, vamos mostrar por indução que 


$$
A^{k} J=J B^{k}
$$

Com efeito, a relação acima se verifica para $k=1$ porque $J$ entrelaça $A$ e $B$, e se for válida para um dado $k \in \mathbb{N}$, então

$$
A^{k+1} J=A^{k} A J=A J B^{k}=J B B^{k}=J B^{k+1}
$$

Como consequência, se $p(t) \in \mathbb{C}[t \in \mathbb{R}]$ é tal que $p(t)=\sum_{1}^{n} \alpha_{i} t^{i}$, então

$$
p(A) J=\sum_{1}^{n} \alpha_{i} A^{i} J=\sum_{1}^{n} \alpha_{i} J B^{i}=J \sum_{1}^{n} \alpha_{i} B^{i}=J p(B)
$$

Dada $f \in \mathrm{C}(\mathbb{R})$, já vimos que é possível encontrar uma sequência $\left(p_{n}\right)_{n \in \mathbb{N}}$ em $\mathbb{C}[t \in \mathbb{R}]$ para o qual

$$
p_{n}(B) \stackrel{p \cdot}{\longrightarrow} f(B) \quad, \quad p_{n}(A) \stackrel{p \cdot}{\longrightarrow} f(A)
$$

e consequentemente

$$
f(A) J \stackrel{p .}{\longleftarrow} p_{n}(A) J=J p_{n}(B) \stackrel{p .}{\longrightarrow} J f(B)
$$

dado que $C$ é limitado. Pela arbitrariedade na escolha de $f$, provamos que

$$
\mathrm{C}(\mathbb{R}) \subseteq \mathcal{M} \doteq\left\{f \in \mathfrak{B}_{\mathrm{c}}(\mathbb{R}): f(A) J=J f(B)\right\}
$$

Usando (essencialmente) o mesmo argumento acima, obtém-se também a condição faltante para que o (T.5) se aplique em $\mathcal{M}$. Isso prova o item a), e por consequência

$$
\begin{aligned}
W^{ \pm}(A, B ; J) & =\mathrm{s}-\lim _{t \rightarrow \mp \infty} e^{\imath t A} J e^{-\imath t B} P_{\mathrm{ac}}(B) \\
& =\mathrm{s}-\lim _{t \rightarrow \mp \infty} J e^{\imath t B} e^{-\imath t B} P_{\mathrm{ac}}(B)=J P_{\mathrm{ac}}(B)
\end{aligned}
$$

donde b).

Visto que a existência de uma relação de entrelaçamento estabelece (de maneira espontânea) a presença de um determinado canal de espalhamento, ponha

$$
\hat{\Psi}(k) \doteq \Psi(k) /\|\Psi(k)\|_{\mathcal{H}}
$$

respeitando a notação do (C.70), e considere

$$
\begin{aligned}
\mathbf{A}: \int_{\mathbb{T}^{2}}^{\oplus} \mathbb{C} \mathrm{d} \varrho \longrightarrow \int_{\mathbb{T}^{2}}^{\oplus} \mathrm{L}^{2}\left(\mathbb{T}^{2}\right) \oplus \mathbb{C} \mathrm{d} \varrho \\
\varphi \mathrm{A} \varphi: \mathbb{T}^{2} \longrightarrow \mathrm{L}^{2}\left(\mathbb{T}^{2}\right) \oplus \mathbb{C} \\
k \longmapsto \varphi(k) \hat{\Psi}(k)
\end{aligned}
$$

Como $\hat{\Psi}$ é contínua e $\varphi \in \mathscr{L}\left(\mathbb{T}^{2}\right)$-mensurável, certamente $\mathbf{A} \varphi \in \mathcal{W}\left(\mathbb{T}^{2}, \mathrm{~L}^{2}\left(\mathbb{T}^{2}\right) \oplus \mathbb{C}\right)$. Além do mais

$$
\int_{\mathbb{T}^{2}}\|\mathbf{A} \varphi(k)\|_{\mathcal{H}}^{2} \mathrm{~d} \varrho(k)=\int_{\mathbb{T}^{2}}|\varphi(k)|^{2}\|\hat{\Psi}(k)\|_{\mathcal{H}}^{2} \mathrm{~d} \varrho(k)=\|\varphi\|_{2}<+\infty
$$


e com isso A encontra-se bem definido. Uma vez checado que este operador é linear, a manipulação acima também indica que ele preserva normas, sendo assim limitado.

Teorema 89. Seja $h_{b} \in\left[0, \frac{1}{2}\right]$. A entrelaça a integral direta dos $\Xi(k)$ com a integral direta dos $\mathrm{E}(k)$. Em símbolos,

$$
\left(\int_{\mathbb{T}^{2}}^{\oplus} \Xi(k) \mathrm{d} \varrho(k)\right) \mathbf{A}=\mathbf{A}\left(\int_{\mathbb{T}^{2}}^{\oplus} \mathrm{E}(k) \mathrm{d} \varrho(k)\right)
$$

Demonstração. De fato, pois para todo $\varphi \in \mathrm{L}^{2}\left(\mathbb{T}^{2}\right)$ e $k \in \mathbb{T}^{2}$, tem-se

$$
\left[\left(\int_{\mathbb{T}^{2}}^{\oplus} \Xi(h) \mathrm{d} \varrho(h)\right) \mathbf{A} \varphi\right](k)=\varphi(k) \Xi(k) \hat{\Psi}(k)=\varphi(k) \mathrm{E}(k) \hat{\Psi}(k)=\left[\mathbf{A}\left(\int_{\mathbb{T}^{2}}^{\oplus} \mathrm{E}(h) \mathrm{d} \varrho(h)\right) \varphi\right](k)
$$

como queríamos demonstrar.

Corolário 90. Seja $h_{b} \in\left[0, \frac{1}{2}\right]$. Então,

$$
W^{ \pm}\left(\int_{\mathbb{T}^{2}}^{\oplus} \Xi(k) \mathrm{d} \varrho(k), \int_{\mathbb{T}^{2}}^{\oplus} \mathrm{E}(k) \mathrm{d} \varrho(k) ; \mathbf{A}\right)=\mathbf{A} P_{\text {ac }}\left(\int_{\mathbb{T}^{2}}^{\oplus} \mathrm{E}(k) \mathrm{d} \varrho(k)\right)
$$

Voltando ao contexto da repulsão eletrônica de alcance zero, ponhamos

$$
\hat{\Psi}^{(\infty)}(k) \doteq \lim _{U_{0} \rightarrow \infty} \hat{\Psi}\left(U_{0}, k\right), \quad k \in \mathbb{T}_{0}^{2}
$$

lembrando que a existência desse limite foi garantida no (T.83) e (C.70). Como $\Psi\left(U_{0}, \cdot\right)$ é contínua (e portanto fracamente mensurável) para todo $U_{0} \geq 0$, teremos que

$$
\hat{\Psi}^{(\infty)} \in \mathcal{W}\left(\mathbb{T}^{2}, L^{2}\left(\mathbb{T}^{2}\right) \oplus \mathbb{C}\right)
$$

Por conta da continuidade da norma, note também que os $\hat{\Psi}^{(\infty)}(k)$ se encontram normalizados, e com isso temos premissas análogas ao caso anterior que justificam a definição do operador linear limitado

$$
\begin{gathered}
\mathbf{A}^{(\infty)}: \int_{\mathbb{T}^{2}}^{\oplus} \mathbb{C} \mathrm{d} \varrho \longrightarrow \int_{\mathbb{T}^{2}}^{\oplus} \mathrm{L}^{2}\left(\mathbb{T}^{2}\right) \oplus \mathbb{C} \mathrm{d} \varrho \\
\varphi \longmapsto \mathbf{A}^{(\infty)} \varphi: \mathbb{T}^{2} \longrightarrow \mathrm{L}^{2}\left(\mathbb{T}^{2}\right) \oplus \mathbb{C} \\
k \longmapsto \varphi(k) \hat{\Psi}^{(\infty)}(k)
\end{gathered}
$$

que a seu respeito vale destacar: 
Teorema 91. Seja $h_{b} \in\left[0, \frac{1}{2}\right]$. Para toda $f \in \mathrm{C}(\mathbb{R})$, são verdadeiras as seguintes afirmações:
a) $\mathrm{s}-\lim _{U_{0} \rightarrow \infty} \mathbf{A}\left(U_{0}\right)=\mathbf{A}^{(\infty)}$
b) s $-\lim _{U_{0} \rightarrow \infty} f\left(\int_{\mathbb{T}^{2}}^{\oplus} \Xi\left(U_{0}, k\right) \mathrm{d} \varrho(k)\right) \mathbf{A}\left(U_{0}\right)=\mathbf{A}^{(\infty)}\left(\int_{\mathbb{T}^{2}}^{\oplus} f \circ \mathrm{E}^{(\infty)}(k) \mathrm{d} \varrho(k)\right)$

Demonstração. Dado $\varphi \in \mathrm{L}^{2}\left(\mathbb{T}^{2}\right)$, para todo $U_{0} \geq 0$ temos

$$
\begin{aligned}
\left\|\mathbf{A}\left(U_{0}\right) \varphi(k)-\mathbf{A}^{(\infty)} \varphi(k)\right\|_{\mathcal{H}}=\left\|\varphi(k) \hat{\Psi}\left(U_{0}, k\right)-\varphi(k) \hat{\Psi}^{(\infty)}(k)\right\|_{\mathcal{H}}= & \\
& =|\varphi(k)| \cdot|| \hat{\Psi}\left(U_{0}, k\right)-\hat{\Psi}^{(\infty)}(k) \|_{\mathcal{H}} \leq 2|\varphi(k)| \quad \varrho \text {-q.t.p }
\end{aligned}
$$

$\log 0$

$$
\left\|\mathbf{A}\left(U_{0}\right) \varphi(\cdot)-\mathbf{A}^{(\infty)} \varphi(\cdot)\right\|_{\mathcal{H}}^{2} \leq 4|\varphi|^{2} \in \mathrm{L}^{1}\left(\mathbb{T}^{2}\right)
$$

e graças ao teorema da convergência dominada,

$$
\lim _{U_{0} \rightarrow \infty}\left\|\mathbf{A}\left(U_{0}\right) \varphi-\mathbf{A}^{(\infty)} \varphi\right\|_{2, \mathcal{H}}^{2}=\lim _{U_{0} \rightarrow \infty} \int_{\mathbb{T}^{2}}\left\|\mathbf{A}\left(U_{0}\right) \varphi(k)-\mathbf{A}^{(\infty)} \varphi(k)\right\|_{\mathcal{H}}^{2} \mathrm{~d} \varrho(k)=0
$$

Agora, como toda $\mathrm{E}\left(U_{0}, \cdot\right)$ é contínua, vide (T.74), e $\mathbb{T}^{2}$ compacto, certamente que

$$
f \circ \mathrm{E}\left(U_{0}, \cdot\right) \in \mathrm{L}^{\infty}\left(\mathbb{T}^{2}\right), \quad U_{0} \geq 0
$$

Além disso, E é crescente na primeira variável, de forma que a família de aplicações acima fica uniformemente limitada pelo valor máximo que $f$ assume no compacto

$$
\left[\min _{k \in \mathbb{T}^{2}} \mathrm{E}(0, k), \max _{k \in \mathbb{T}^{2}} \mathfrak{b}(k)\right]
$$

Aplicando a (P.37) junto do (T.83) e da continuidade de $f$, deduzimos então que

$$
\lim _{U_{0} \rightarrow \infty}\left(\int_{\mathbb{T}^{2}}^{\oplus} f \circ \mathrm{E}\left(U_{0}, k\right) \mathrm{d} \varrho(k)\right) \varphi=\left(\int_{\mathbb{T}^{2}}^{\oplus} f \circ \mathrm{E}^{(\infty)}(k) \mathrm{d} \varrho(k)\right) \varphi
$$

de onde

$$
\begin{aligned}
\lim _{U_{0} \rightarrow \infty} f\left(\int_{\mathbb{T}^{2}}^{\oplus} \Xi\left(U_{0}, k\right) \mathrm{d} \varrho(k)\right) \mathbf{A}\left(U_{0}\right) \varphi & =\lim _{U_{0} \rightarrow \infty}\left(\int_{\mathbb{T}^{2}}^{\oplus} f\left(\Xi\left(U_{0}, k\right)\right) \mathrm{d} \varrho(k)\right) \mathbf{A}\left(U_{0}\right) \varphi= \\
& =\lim _{U_{0} \rightarrow \infty} \mathbf{A}\left(U_{0}\right)\left(\int_{\mathbb{T}^{2}}^{\oplus} f \circ \mathrm{E}\left(U_{0}, k\right) \mathrm{d} \varrho(k)\right) \varphi=\mathbf{A}^{(\infty)}\left(\int_{\mathbb{T}^{2}}^{\oplus} f \circ \mathrm{E}^{(\infty)}(k) \mathrm{d} \varrho(k)\right) \varphi
\end{aligned}
$$

seja qual for $\varphi \in \mathrm{L}^{2}\left(\mathbb{T}^{2}\right)$, em virtude de um fato situado no apêndice A.

Com isso damos sentido a dinâmica do sistema no limite de hard-core como sendo a dinâmica de uma partícula efetiva com dispersão $\mathrm{E}^{(\infty)}$. Precisamente: 
Corolário 92. Seja $h_{b} \in\left[0, \frac{1}{2}\right]$. Então,

$$
\mathrm{s}-\lim _{U_{0} \rightarrow \infty} \exp \left(-\imath t \int_{\mathbb{T}^{2}}^{\oplus} \Xi\left(U_{0}, k\right) \mathrm{d} \varrho(k)\right) \mathbf{A}\left(U_{0}\right)=\mathbf{A}^{(\infty)}\left(\int_{\mathbb{T}^{2}}^{\oplus} e^{-\imath t \mathrm{E}^{(\infty)}(k)} \mathrm{d} \varrho(k)\right)
$$

Em particular, o resultado mais relevante desta dissertação prevê a estabilidade do canal de pareamento $d$-wave com relação ao limite de hard-core:

Corolário 93. Seja $h_{b} \in\left[0, \frac{1}{2}\right]$. Se ve é real analítica no interior de seu domínio, então

$$
\mathrm{s}-\lim _{U_{0} \rightarrow \infty} W^{ \pm}\left(\int_{\mathbb{T}^{2}}^{\oplus} \Xi\left(U_{0}, k\right) \mathrm{d} \varrho(k), \int_{\mathbb{T}^{2}}^{\oplus} \mathrm{E}\left(U_{0}, k\right) \mathrm{d} \varrho(k) ; \mathbf{A}\left(U_{0}\right)\right)=\mathbf{A}^{(\infty)}
$$

Demonstração. A dispersão E será real analítica no interior de seu domínio em função do (T.74), daí

$$
P_{\mathrm{ac}}\left(\int_{\mathbb{T}^{2}}^{\oplus} \mathrm{E}\left(U_{0}, k\right) \mathrm{d} \varrho(k)\right)=\mathbb{1}, \quad U_{0} \geq 0
$$

conforme o (Ex.42), e portanto

$$
\mathrm{s}-\lim _{U_{0} \rightarrow \infty} W^{ \pm}\left(\int_{\mathbb{T}^{2}}^{\oplus} \Xi\left(U_{0}, k\right) \mathrm{d} \varrho(k), \int_{\mathbb{T}^{2}}^{\oplus} \mathrm{E}\left(U_{0}, k\right) \mathrm{d} \varrho(k) ; \mathbf{A}\left(U_{0}\right)\right)=\mathrm{s}-\lim _{U_{0} \rightarrow \infty} \mathbf{A}\left(U_{0}\right)=\mathbf{A}^{(\infty)}
$$

pelo (C.90) junto ao (T.91). 


\section{Apêndice A}

\section{Resultados Suplementares}

Resultado 1. Dado $\Lambda$ um conjunto arbitrário e $f: \Lambda \longrightarrow[0, \infty]$, tem-se

$$
\int_{\Lambda} f \mathrm{~d} \mathfrak{c}=\sum_{\lambda \in \Lambda} f(\lambda)
$$

Demonstração. Ver [Fol13].

Resultado 2. Dado $\Lambda$ um conjunto qualquer e $f: \Lambda \longrightarrow[0, \infty]$, ponha $\mathcal{A} \doteq f^{-1}(0, \infty]$. São verdadeiras as seguintes afirmações:

a) Se $\mathcal{A}$ é não-enumerável, então $\sum_{\lambda \in \Lambda} f(\lambda)=\infty$.

b) Se $\mathcal{A}$ possui uma infinidade enumerável de elementos, então $\sum_{1}^{\infty} f(\varphi(n))=\sum_{\lambda \in \Lambda} f(\lambda)$ seja qual for $\varphi$ enumeração de $\mathcal{A}$.

Demonstração. Ver [Fol13].

Resultado 3. Seja $(X, \Omega, \mu)$ um espaço de medida semifinito. Então

$$
\begin{aligned}
& \Sigma: \mathrm{L}^{\infty}(X, \Omega, \mu) \longrightarrow \mathrm{L}^{1}(X, \Omega, \mu)^{*} \\
& g \quad \longmapsto \quad \Sigma_{g} \quad: \quad \mathrm{L}^{1}(X, \Omega, \mu) \longrightarrow \quad \mathbb{C} \\
& f \quad \longmapsto \int_{X} g(x) f(x) \mathrm{d} \mu(x)
\end{aligned}
$$

é uma transformação linear $\left(\|\cdot\|_{\infty},\|\cdot\|_{\text {op }}\right)$-isométrica.

Demonstração. Ver [Fol13].

Resultado 4. O grupo alternante $\mathcal{A}_{n}$ tem ordem $\frac{n !}{2}$.

Demonstração. Como $\mathcal{S}_{n} \ni \sigma \stackrel{\varphi}{\longrightarrow} \operatorname{sgn}(\sigma) \in\{-1,1\}$ define um homomorfismo entre o grupo simétrico e o grupo multiplicativo, o primeiro teorema do homomorfismo garante que $\mathcal{A}_{n}=\operatorname{ker}(\varphi) \triangleleft \mathcal{S}_{n}$, valendo 


$$
\frac{\mathcal{S}_{n}}{\mathcal{A}_{n}}=\frac{\mathcal{S}_{n}}{\operatorname{ker}(\varphi)} \simeq \operatorname{Im}(\varphi)=\{-1,1\}
$$

Mas sendo $\mathcal{S}_{n}$ finito, o teorema de Lagrange se aplica, e portanto

$$
\frac{n !}{\left|\mathcal{A}_{n}\right|}=\frac{\left|\mathcal{S}_{n}\right|}{\left|\mathcal{A}_{n}\right|}=\left[\mathcal{S}_{n}: \mathcal{A}_{n}\right]=\left|\frac{\mathcal{S}_{n}}{\mathcal{A}_{n}}\right|=|\{-1,1\}|=2 \Longrightarrow\left|\mathcal{A}_{n}\right|=\frac{n !}{2}
$$

como queríamos.

Resultado 5. Para todo $x \in \mathbb{R}$, vale a seguinte identidade:

$$
\left|\frac{e^{\imath x}-1}{x}\right| \leq 1
$$

Demonstração. Para todo $t \in(0,+\infty)$, o teorema do valor médio nos oferta $c_{t} \in(0, t)$ de modo que

$$
\frac{\sin (t)}{t}=\frac{\sin (t)-\sin (0)}{t-0}=\cos \left(c_{t}\right) \leq 1
$$

Consequentemente

$$
\left|e^{i x}-1\right|^{2}=(\cos (x)-1)^{2}+\sin ^{2}(x)=2(1-\cos (x))=(2 \sin (x / 2))^{2} \leq x^{2}
$$

se $x \geq 0, \mathrm{e}$

$$
\left|e^{\imath x}-1\right|=\left|e^{-\imath x}-1\right| \leq(-x)^{2}=x^{2}
$$

caso contrário. De qualquer forma, a tese se verifica.

Resultado 6. Seja $\mathfrak{H}$ um espaço de Hilbert e $\left\{B_{i}\right\}_{i \in \mathbb{N}}$ uma família em $\mathcal{B}(\mathfrak{H})$ tal que

$$
\lim _{i \rightarrow \infty}\left\langle u, B_{i} v\right\rangle_{\mathfrak{H}} \quad \forall u, v \in \mathfrak{H}
$$

Logo, existe um único $B \in \mathcal{B}(\mathfrak{H})$ de forma que

$$
\lim _{i \rightarrow \infty}\left\langle u, B B_{i} v\right\rangle_{\mathfrak{H}}=\langle u, B v\rangle_{\mathfrak{H}} \quad \forall u, v \in \mathfrak{H}
$$

Demonstração. Ver [RS80].

Resultado 7. Seja $\mathfrak{H}$ um espaço de Hilbert, $B \in \mathcal{B}(\mathfrak{H})$ autoadjunto e $c$ uma constante real positiva de modo que

$$
0<c \leq\|B \psi\|_{\mathfrak{H}} \quad \forall \psi \in \mathbb{S}^{1}(\mathfrak{H})
$$

Logo, $B$ é inversível.

Demonstração. Como $B \psi \neq 0$ seja qual for $\psi \in \mathbb{S}^{1}(\mathfrak{H})$, de início já sabemos que $B$ é injetivo. Também provém das hipóteses que

$$
c\|\psi\|_{\mathfrak{H}} \leq\|B \psi\|_{\mathfrak{H}} \quad \forall \psi \in \mathfrak{H}
$$


Se $\left(B \psi_{n}\right)_{n \in \mathbb{N}}$ é uma sequência de Cauchy, então para um dado $\varepsilon>0$, nos é fornecido $n_{0} \in \mathbb{N}$ de forma que

$$
c|| \psi_{m}-\psi_{n}\left\|_{\mathfrak{H}} \leq\right\| B\left(\psi_{m}-\psi_{n}\right)\left\|_{\mathfrak{H}}=\right\| B \psi_{m}-B \psi_{n}\left\|_{\mathfrak{H}}<c \cdot \varepsilon \Longrightarrow\right\| \psi_{m}-\psi_{n} \|_{\mathfrak{H}}<\varepsilon
$$

sempre que $m, n>n_{0}$. Isso prova que $\left(\psi_{n}\right)_{n \in \mathbb{N}}$ também é de Cauchy, e logo convergente. Aplicando a continuidade de $B$, deduzimos que $\left(B \psi_{n}\right)_{n \in \mathbb{N}}$ é convergente, e $\operatorname{Im}(B)$ por sua vez completo. Mas daí

$$
\operatorname{Im}(B)=\overline{\operatorname{Im}(B)}=\operatorname{Ker}\left(B^{*}\right)^{\perp}=\operatorname{Ker}(B)^{\perp}=\{0\}^{\perp}=\mathfrak{H}
$$

e estamos conversados.

Resultado 9. Seja $R$ um bloco $n$-dimensional fechado não degenerado ${ }^{1}$ e $f \in \mathrm{C}(R)$. São verdadeiras as seguintes alegações:

a) $\operatorname{ess} \operatorname{im}(f)=\operatorname{Im}(f)$

b) $\operatorname{ess} \sup (f)=\max _{R}|f|$

Demonstração. Para todo $\varepsilon>0$ e $y$ na imagem de $f, f^{-1}\left(B_{\varepsilon}(y)\right)$ tem medida de Lebesgue estritamente positiva pois graças a continuidade de $f$, é um aberto não vazio de $R^{2}$. Reciprocamente, $\operatorname{sendo} \operatorname{Im}(f)$ compacto (logo fechado) por causa da continuidade de $f$,

$$
y \notin \operatorname{Im}(f) \Longrightarrow \exists r>0: B_{r}(y) \cap \operatorname{Im}(f)=\varnothing \Longrightarrow f^{-1}\left(B_{r}(y)\right)=\varnothing \Longrightarrow y \notin \operatorname{essim}(f)
$$

Para o item b), veja [Fol13].

Resultado 8. Seja $X, y, z \mathbb{K}$-espaços vetoriais normados, $\{X(t)\}_{t \in \mathbb{R}}$ uma família em $\mathcal{B}(X, y)$ e $\{Y(t)\}_{t \in \mathbb{R}}$ outra em $\mathcal{B}(y, z)$ limitada. Se

$$
\lim _{t \pm \infty} X(t) x=X x \quad, \quad \lim _{t \pm \infty} Y(t) y=Y y
$$

para todo $x \in \mathcal{X}$ e $y \in \mathcal{y}$, então

$$
\lim _{t \pm \infty} Y(t) X(t) x=Y X x
$$

para todo $x \in \mathcal{X}$.

Demonstração. Claro, pois se $M>0$ limita da norma de operador dos $Y(t)$, então

$$
\begin{aligned}
\|Y(t) X(t) x-Y X x\|_{z} \leq\|Y(t) X(t) x-Y(t) X x\|_{z}+\|Y(t) X x-Y X x\|_{z} & \leq \\
& \leq M\|X(t) x-X x\|_{y}+\|Y(t) X x-Y X x\|_{z}
\end{aligned}
$$

para todo $t$ real e $x \in \mathcal{X}$.

\footnotetext{
${ }^{1}$ Isto é, $R$ se exprime como produto cartesiano de $n$ intervalos fechados e não degenerados de $\mathbb{R}$.

${ }^{2}$ Se $R$ fosse um compacto qualquer de $\mathbb{R}$, não seria verdade que todo aberto não vazio de $R$ tem medida estritamente positiva.
} 
- $\Omega$ definido na seção 4.4 qualifica um aberto de $\mathbb{R}^{3}$.

Dado $\left(k_{0}, x_{0}\right) \in \Omega$, seja $r>0$ de forma que $\mathbf{B}_{r}\left(k_{0}\right) \subseteq \operatorname{int}\left(\mathbb{T}_{0}^{2}\right)$ e $\varepsilon>0$ tal que todo operador limitado na bola de centro $\Xi_{1,1}\left(k_{0}\right)-x_{0} \mathbb{1}$ e raio $\varepsilon$ seja inversível. A continuidade de $\Xi_{1,1}$ nos fornece $\delta>0$ de tal modo que

$$
k \in \mathbb{T}^{2}:\left|k-k_{0}\right|<\delta \Longrightarrow\left\|\Xi_{1,1}(k)-\Xi_{1,1}\left(k_{0}\right)\right\|_{\text {op }}<\frac{\varepsilon}{2}
$$

Pondo $s \doteq \min \{\varepsilon / 2, \delta, r\}>0$, obtemos

$$
\begin{aligned}
\|\left(\Xi_{1,1}(k)-x \mathbb{1}\right) & -\left(\Xi_{1,1}\left(k_{0}\right)-x_{0} \mathbb{1}\right)\left\|_{\text {op }} \leq\right\|\left(\Xi_{1,1}(k)-x \mathbb{1}\right)-\left(\Xi_{1,1}(k)-x_{0} \mathbb{1}\right) \|_{\text {op }}+ \\
& +\left\|\left(\Xi_{1,1}(k)-x_{0} \mathbb{1}\right)-\left(\Xi_{1,1}\left(k_{0}\right)-x_{0} \mathbb{1}\right)\right\|_{\text {op }}=\left|x-x_{0}\right|+\left.\left\|\Xi_{1,1}(k)-\Xi_{1,1}\left(k_{0}\right)\right\|\right|_{\text {op }}<\varepsilon
\end{aligned}
$$

sempre que

$$
(k, x) \in \mathbb{R}^{3}:\left|x-x_{0}\right|+\left|k-k_{0}\right|=\left|(k, x)-\left(k_{0}, x_{0}\right)\right|<s
$$

Em síntese,

$$
(k, x) \in \mathbf{B}_{s}\left(k_{0}, x_{0}\right) \Longrightarrow\left\{\begin{array}{l}
\Xi_{1,1}(k)-x \mathbb{1} \in \mathbf{B}_{\varepsilon}\left(\Xi_{1,1}\left(k_{0}\right)-x_{0} \mathbb{1}\right) \Longrightarrow x \in \mathcal{R}\left(\Xi_{1,1}(k)\right) \\
k \in \mathbf{B}_{r}\left(k_{0}\right) \Longrightarrow k \in \operatorname{int}\left(\mathbb{T}_{0}^{2}\right)
\end{array}\right.
$$

e daí o raio da bola (aberta) de $\mathbb{R}^{3}$ que testemunha $\left(k_{0}, x_{0}\right)$ como um ponto interior de $\Omega$.

\section{- Limitação da parte fermiônica do Hamiltoniano (de volume infinito)}

Primeiramente, vejamos que as transformações encarregadas de mapear

$$
\delta_{(p, t)} \wedge \delta_{(q, r)} \stackrel{Q_{z}}{\longmapsto} \delta_{(p+z, t)} \wedge \delta_{(q, r)}+\delta_{(p, t)} \wedge \delta_{(q+z, r)} \quad, \quad \delta_{(p, t)} \wedge \delta_{(q, r)} \stackrel{R}{\longmapsto} \mathrm{U}(p-q) \delta_{(p, t)} \wedge \delta_{(q, r)}
$$

são limitadas. Com efeito, se $\mathcal{E}$ é uma base ortonormal do espaço de dois elétrons como na (P.46), existem operadores $Q_{z}$ e $R$ definidos em $\langle\mathcal{E}\rangle$ cumprindo as setas acima ao menos nos vetores de $\mathcal{E}$. Contudo, mesmo que $\delta_{(q, r)} \wedge \delta_{(p, t)}$ não conste em $\mathcal{E}$, certamente $\delta_{(p, t)} \wedge \delta_{(q, r)} \in \mathcal{E}$, e daí

$$
\begin{aligned}
Q_{z}\left(\delta_{(q, r)} \wedge \delta_{(p, t)}\right)=-Q_{z}\left(\delta_{(p, t)} \wedge\right. & \left.\delta_{(q, r)}\right)=-\delta_{(p+z, t)} \wedge \delta_{(q, r)}-\delta_{(p, t)} \wedge \delta_{(q+z, r)}= \\
& =\delta_{(q, r)} \wedge \delta_{(p+z, t)}+\delta_{(q+z, r)} \wedge \delta_{(p, t)}=\delta_{(q+z, r)} \wedge \delta_{(p, t)}+\delta_{(q, r)} \wedge \delta_{(p+z, t)}
\end{aligned}
$$

Do mesmo modo,

$$
R\left(\delta_{(q, r)} \wedge \delta_{(p, t)}\right)=-R\left(\delta_{(p, t)} \wedge \delta_{(q, r)}\right)=-\mathrm{U}(p-q) \delta_{(p, t)} \wedge \delta_{(q, r)}=\mathrm{U}(q-p) \delta_{(q, r)} \wedge \delta_{(p, t)}
$$

e a existência de tais operadores encontra-se devidamente justificada. $R$ também é limitado pois além de preservar a ortogonalidade de $\mathcal{E}$, verifica 


$$
\left\|R\left(\delta_{(p, t)} \wedge \delta_{(q, r)}\right)\right\|_{\wedge}=|\mathrm{U}(p-q)| \cdot\left\|\delta_{(p, t)} \wedge \delta_{(q, r)}\right\|_{\wedge} \leq \sup _{\mathbb{Z}^{2}}|\mathrm{U}|, \quad \delta_{(p, t)} \wedge \delta_{(q, r)} \in \mathcal{E}
$$

porque sendo $\mathbb{Z}^{2}$-somável, U também é limitada. Resta provar que $Q_{z}$ é limitado. De fato, sabemos que $\mathcal{G} \doteq-\mathcal{E}$ também define uma base ortonormal do espaço de dois elétrons, e por isso existem operadores $Q_{z}^{\mathcal{E}}$ (respec. $Q_{z}^{\mathcal{G}}$ ) cumprindo

$$
\delta_{(p, t)} \wedge \delta_{(q, r)} \longmapsto \delta_{(p+z, t)} \wedge \delta_{(q, r)}
$$

nos vetores de $\mathcal{E}$ (respec. $\mathcal{G}) . Q_{z}^{\mathcal{E}}$ é limitado pois preserva a ortogonalidade de $\mathcal{E}$ e verifica

$$
\left\|Q_{z}^{\mathcal{E}}\left(\delta_{(p, t)} \wedge \delta_{(q, r)}\right)\right\|_{\wedge}=\left\|\delta_{(p+z, t)} \wedge \delta_{(q, r)}\right\|_{\wedge} \leq 1, \quad \delta_{(p, t)} \wedge \delta_{(q, r)} \in \mathcal{E}
$$

não havendo garantia de que $(p+z, t)$ seja distinto de $(q, r)$. Similarmente, $Q_{z}^{\mathcal{G}}$ também é limitado, e portanto $Q_{z}=Q_{z}^{\mathcal{E}}+Q_{z}^{\mathcal{G}}$. Dito isto, $H_{f}$ se estende de modo único a um operador limitado definido em todo o espaço de 2 elétrons, igualmente denotado por $H_{f}$. 


\section{Referências Bibliográficas}

\section{Artigos e Periódicos}

[BdPdSP15] Jean-Bernard Bru, Antonio Delgado de Pasquale, and Walter de Siqueira Pedra. d-wave pairing driven by bipolaric modes related to giant electron-phonon anomalies in high-tc superconductors. Journal of Statistical Mechanics: Theory and Experiment, 2015(3):P03002, 2015. https://arxiv.org/pdf/1610.07416.pdf. (Último acesso em 05/03/2020).

[BdSPDdP19] Jean-Bernard Bru, Walter de Siqueira Pedra, and Antonio Delgado de Pasquale. Isotropic bipolaron-fermion exchange theory and unconventional pairing in cuprate superconductors. Annalen der Physik, 531(1):1700235, 2019. http://kleine.mat.uniroma3.it/mp_arc/c/ 17/17-58.pdf. (Último acesso em 05/03/2020).

[DD06] J Hecker Denschlag and AJ Daley. Exotic atom pairs: Repulsively bound states in an optical lattice. arXiv preprint cond-mat/0610393, 2006. https://arxiv.org/pdf/cond-mat/ 0610393.pdf. (Último acesso em 05/03/2020).

[WTL ${ }^{+}$06] K Winkler, G Thalhammer, F Lang, R Grimm, J Hecker Denschlag, AJ Daley, A Kantian, HP Büchler, and $\mathrm{P}$ Zoller. Repulsively bound atom pairs in an optical lattice. Nature, 441(7095):853-856, 2006. https : //arxiv.org/pdf/cond-mat/0605196.pdf. (Último acesso em 05/03/2020).

\section{Livros}

[Fol13] Gerald B Folland. Real analysis: modern techniques and their applications. John Wiley \& Sons, 2013.

[GS11] Stephen J Gustafson and Israel Michael Sigal. Mathematical concepts of quantum mechanics. Springer Science \& Business Media, 2011.

[HS12] Peter D Hislop and Israel Michael Sigal. Introduction to spectral theory: With applications to Schrödinger operators, volume 113. Springer Science \& Business Media, 2012.

[Lim76] Elon Lages Lima. Curso de Análise, vol. 2. 1976.

[Lim83] Elon Lages Lima. Espaços métricos, volume 4. Instituto de Matemática Pura e Aplicada, CNPq Rio de Janeiro, 1983.

[Nie80] Ole A Nielsen. Direct integral theory, volume 61. CRC Press, 1980. 
[RS75] Michael Reed and Barry Simon. Methods of modern mathematical physics. II. Fourier analysis, self-adjointness. Academic Press [Harcourt Brace Jovanovich Publishers], New York, 1975.

[RS78] Michael Reed and Barry Simon. Methods of modern mathematical physics. IV. Analysis of operators. Academic Press [Harcourt Brace Jovanovich Publishers], New York, 1978.

[RS79] Michael Reed and Barry Simon. Methods of modern mathematical physics. III. Academic Press [Harcourt Brace Jovanovich Publishers], New York, 1979.

[RS80] Michael Reed and Barry Simon. Methods of modern mathematical physics. I. Academic Press Inc. [Harcourt Brace Jovanovich Publishers], New York, second edition, 1980.

[Wer06] Dirk Werner. Funktionalanalysis. Springer, 2006.

\section{Teses e Dissertações}

$\left[\mathrm{P}^{+} 18\right] \quad$ Antonio Maria Sexto Ysaias Delgado Pasquale et al. Existência de pares d-wave e ondas de densidade em uma classe de modelos microscópicos para supercondutores com alta temperatura de transição. PhD thesis, Universidade de São Paulo, 2018. https://teses.usp.br/ teses/disponiveis/43/43134/tde-03052018-150652/publico/Tesis.pdf. (Último acesso em 05/03/2020).

\section{Notas de Aula e Apostilas}

[Att] Stéphane Attal. Fock spaces. http://math.univ-lyon1.fr/ attal/Fock_Spaces.pdf. (Último acesso em 06/01/2019). 